\title{
First-Row Transition Metal (De)hydrogenation Catalysis based on Functional Pincer Ligands
}

\author{
Lukas Alig ${ }^{\ddagger}$, Maximilian Fritz ${ }^{\ddagger}$ Sven Schneider* \\ Universität Göttingen, Institut für Anorganische Chemie, Tammannstrasse 4, D-37077 Göttingen, Germany
}

ABSTRACT: The use of 3d metals in de-/hydrogenation catalysis has emerged as a competitive field with respect to 'traditional' precious metal catalyzed transformations. The introduction of functional pincer ligands that can store protons and/or electrons as expressed by metal-ligand cooperativity and ligand redox-activity strongly stimulated this development as conceptual starting point for rational catalyst design. This reviews aims at providing a comprehensive picture of the utilization of functional pincer ligands in first-row transition metal hydrogenation and dehydrogenation catalysis and related synthetic concepts relying on these such as the hydrogen borrowing methodology. Particular emphasis is put on the implementation and relevance of cooperating and redox-active pincer ligands within the mechanistic scenarios.

\section{CONTENTS}

\section{Introduction}

1.1. Metal-ligand Cooperativity (MLC)

1.2. Redox active ligands

\section{Privileged Pincer Platforms}

2.1. Aminopincer Platforms

2.1.1. Diphosphinoamines and Related Ligands

2.1.2. BPI and Boxmi Ligands

2.1.3. Aminophenol Pincer Ligands

2.1.4. Dihydrazonopyrroles

2.2. Pyridyl Pincer Ligands

2.2.1. Diphosphinopyridine and Related Ligands

2.2.2. Pyridinediimines and Related Ligands

2.2.3. Terpyridine

2.2.4. Pybox

2.2.5. Pyridyldicarbenes

2.2.6. Iminopyridine and Related Systems

2.2.7. Bipyridine Based Pincer Ligands

2.2.8. Azaphenyl Ligands and Related Systems

2.2.9. Bis(pyrazolyl)pyridine

2.2.10. Phosphaalkene Pincer Ligands

\section{Hydrogenation}

3.1. Hydrogenation of Ketones and Aldehydes

3.1.1. Pyridyl-Based and Related Pincer Catalysts

3.1.2. Dialkylamino Pincer Catalysts

3.1.3. Asymmetric Hydrogenation of Ketones

3.2. Hydrogenation of Imines and $\mathrm{N}$-Heterocycles

3.3. Hydrogenation of Amides
3.4. Hydrogenation of Nitriles

3.5. Hydrogenation of Esters

3.6. Hydrogenation of $\mathrm{CO}_{2}$

3.6.1. Pyridyl-Based Pincer Catalysts

3.6.2. Diphosphinoamine Pincer Catalysts

3.7. Hydrogenation of Olefins

3.7.1. Pyridyl-Based Pincer Catalysts

3.7.2. Aminodiphosphine and Related Pincer

Catalysts

3.8. Hydrogenation of Alkynes

\section{Dehydrogenation}

4.1. (Acceptorless) Alcohol Dehydrogenation

4.1.1. Dialkylamino Pincer Catalysts

4.1.2. Pyridyl-Based and Related Pincer Catalysts

4.2. Formic Acid Dehydrogenation

4.3. Dehydrogenation of Nitrogen-Containing Substrates

4.3.1. Amineboranes

4.3.2. $\mathrm{N}$-Heterocycles

4.3.3. Dehydrogenative C-N Coupling

4.3.4. Dehydrogenative Aminomethylation

\section{Hydrogen Borrowing Methodology}

6. Outlook 


\section{INTRODUCTION}

First-row transition metal compounds have emerged in recent years as homogeneous catalysts for numerous organic transformations, ${ }^{1-19}$ such as de-/hydrogenation, ${ }^{18,20-33}$ and other hydroelementation ${ }^{34-38}$ reactions that were traditionally the realm of precious metal catalysis. The development of base metal hydrogenation catalysts also inspired sustainable concepts for the controlled release and fixation of dihydrogen within chemical energy storage schemes. ${ }^{32,39-46}$ Due to the generally lower toxicity compared with $4 \mathrm{~d}$ and $5 \mathrm{~d}$ metal complexes, ${ }^{47}$ the use of $3 \mathrm{~d}$ metals is less environmentally harmful meeting several principles of Green Chemistry. Furthermore, $3 \mathrm{~d}$ metals are considerably more abundant in the continental crust than their heavier $4 \mathrm{~d} / 5 \mathrm{~d}$ homologues, ranging from scandium (16 ppm) to iron (43200 ppm), the fourth most abundant element (Figure 1$){ }^{48}$ In contrast, some $4 \mathrm{~d}$ metals $(\mathrm{Ru}, \mathrm{Rh}, \mathrm{Pd})$ and most of the $5 \mathrm{~d}$ metals (in particular Re, Os, Ir and $\mathrm{Pt}$ ) are rare elements with limited deposits suitable for today's mining technology.

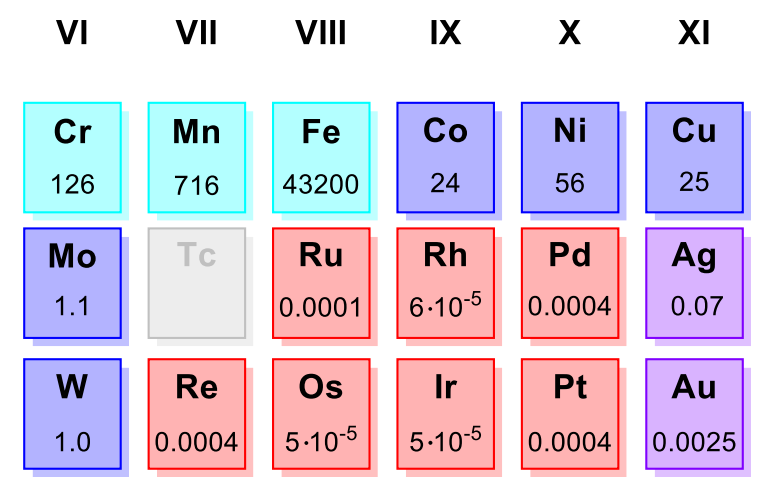

Figure 1. Abundance of mid to late transition metals in the earth's continental crust (concentration in ppm). ${ }^{48}$

The increasing demand and cost for noble metal precursors sparked a rising interest in their replacement for more abundant homogeneous catalysts. However, the robustness (expressed in the turnover number TON) and the activity (expressed in the turnover frequency TOF) of base metal catalysts often lacks behind the performance of noble metal catalysts. The emergence of base metal catalysis was therefore strongly driven by the development of sophisticated ligand systems as an enabling methodology. The proportional cost of such complex ligand platforms for $3 \mathrm{~d}$ metal catalysis with high activity and selectivity, should not be underestimated. Besides cost considerations, the field of base metal catalysis is therefore also driven by catalytic transformations that have not been realized with precious metal catalysts before.

'Pincer' ligands were introduced by Shaw in $1976 .{ }^{49}$ According to the early definition by van Koten and Albrecht, pincer ligands are tridentate ligands with a central, $\sigma$-donating phenyl moiety that carries two lateral amino- or phosphinomethyl $\mathrm{CH}_{2} \mathrm{ER}_{2}(\mathrm{E}=\mathrm{N}, \mathrm{P})$ side groups in ortho-position. ${ }^{50}$ As a result of the chelating, rigid binding mode, transition metal complexes often exhibit high thermal stability and well defined reactivity at the remaining coordination sites, which has been extensively exploited in catalysis and many other application., ${ }^{2,32,51-60}$ However, the term 'pincer ligand' has since been considerably widened to a point where a precise definition is blurred by the inclusion of many meridionally binding, mono-, di- and tri-anionic and even neutral tridentate ligands that cover a vast range of central and flanking coordinating groups and 'backbones' linkers connecting them. This structural and electronic variability allows for versatile fine-tuning of chemical properties, which strongly contributed to the success of the pincer ligand concept. ${ }^{54,61-64}$ Importantly, the conceptual utilization of functional pincer ligands decisively stimulated base metal catalyzed de-/hydrogenation catalysis.

Scheme 1. Schematic representations of typical hydrogenation cycles of olefins and carbonyl groups with and without formal metal redox changes. ${ }^{65,81}$

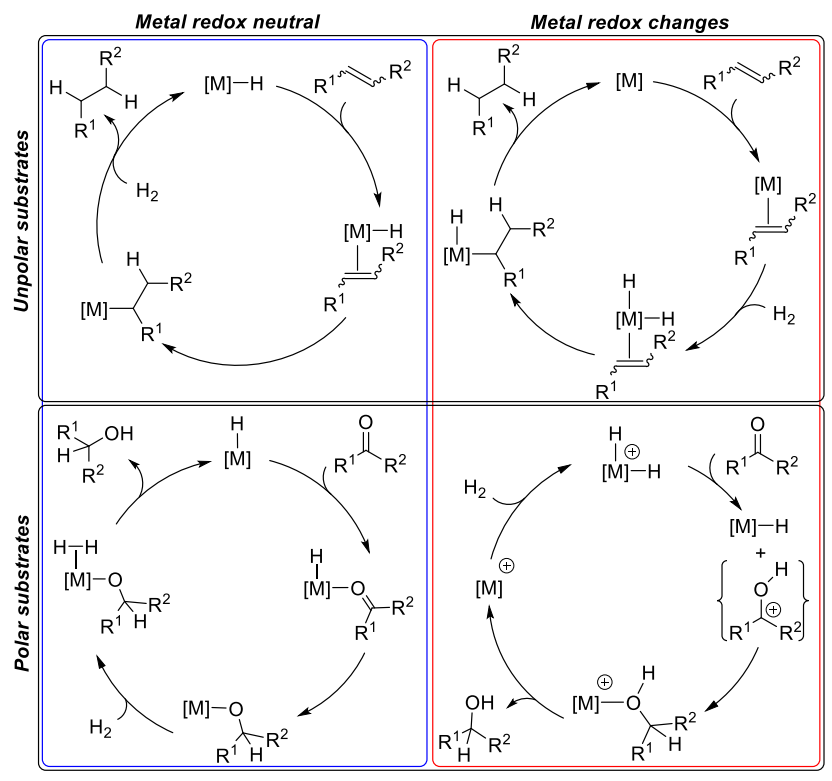

The parameters that contribute to the activity of $3 \mathrm{~d}$ metal hydrogenation catalysts with respect to their heavier homologues were recently discussed by Zell and Langer. ${ }^{65}$ Within a strongly simplified picture, two 'conventional' mechanistic scenarios are well established for hydrogenation of olefins and carbonyl groups, respectively (Scheme 1): The dihydride mechanisms (right) imply substrate binding, stepwise hydrogen transfer to the substrate and $\mathrm{H}_{2}$ oxidative addition, associated with formal metal \pm 2 redox steps. Within monohydride mechanism (left), metal redox events are avoided by $\mathrm{H}_{2}$ heterolysis with proton transfer from a dihydrogen ligand to alkyl (olefin hydrogenation) or alkoxy (carbonyl hydrogenation) ligands. These considerations define the relevant parameters that determine the thermochemical framework for comparing $3 \mathrm{~d} v s 4 / 5 \mathrm{~d}$ metal catalysts, i.e. (1) substrate binding constants (2) $\mathrm{M}-\mathrm{H}$ vs. $\mathrm{M}-\mathrm{C} / \mathrm{O}$ bond strength as expressed by bond dissociation free energies (BDFE) for homolytic processes and $\mathrm{M}-\mathrm{H}$ hydricity $\left(\Delta G_{\mathrm{H}^{-}}\right)$ and $\mathrm{p} K_{\mathrm{a}}$ for hydride or proton transfer, respectively, and (3) acidity of the dihydrogen intermediate as expressed by $\mathrm{p} K_{\mathrm{a}}\left(\mathrm{M}-\mathrm{H}_{2}\right) .{ }^{66-68}$ These parameters will be discussed in the next sections in the context of two ligand design principles that have been instrumental in the emergence of first-row 
metal catalysis, i.e. the introduction of redox active pincer ligands $27,69-74,78,142-145$ and metal-ligand cooperativity. ${ }^{26,27,30,32,39,46,51,77,93}$

This review aims at covering the application of functional pincer ligands in $3 \mathrm{~d}$ metal catalyzed de-/hydrogenation. The term 'functional ligand' has been used for a variety of different phenomena of active ligand participation both in the first and second coordination sphere around the metal. ${ }^{78}$ Within this review on de-/hydrogenation catalysis we will focus on redox active and cooperating pincer ligands to discuss the relevance of reversible reservoirs for protons and/or electrons in pivotal organic applications of proton coupled electron transfer. Both principles have been separately reviewed. $26,27,30,32,39,46,51,69-74,77,78,93,142-145$ However, the direct comparison offers additional insights for catalyst design.

Scheme 2. Simplified, schematic representations of redox-active, cooperating and chemically non-innocent pincer ligands. ${ }^{203}$

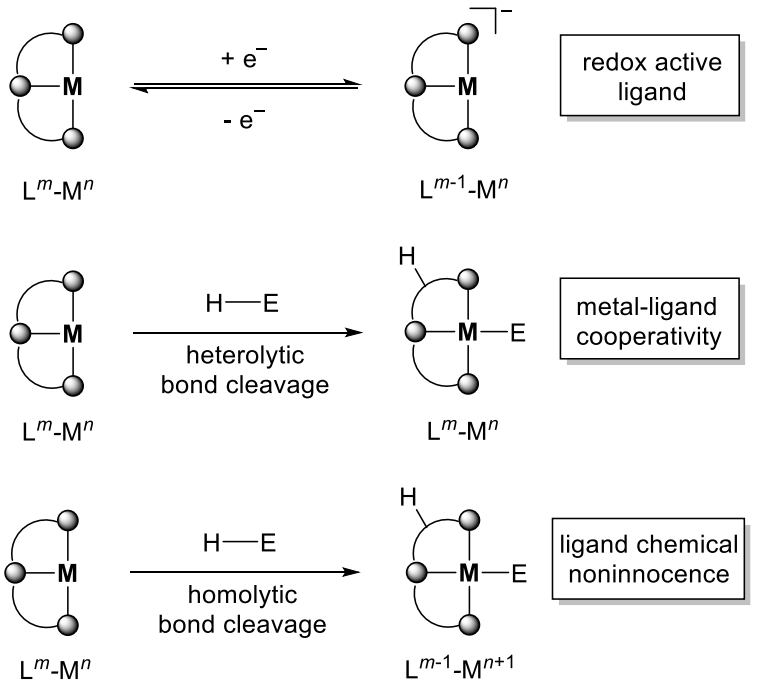

\subsection{Metal-ligand Cooperativity (MLC)}

As a general trend, first row transition metal hydrides exhibit slightly lower M-H BDFEs compared with analogous heavier analogues by approximately 5-10 kcal mol ${ }^{-1} \cdot{ }^{79,80}$ Furthermore, exchange of ligands, such as $\mathrm{CO}$ for phosphines or phosphites had negligible effects on the $\mathrm{M}-\mathrm{H}$ BDFE within error. As judged from the rather limited body of systematic data, first row transition metal hydride BFFEs seem to be at the lower end for what is required for $\mathrm{H}_{2}(\mathrm{BDFEMeCN}$ $=102 \mathrm{kcal} \mathrm{mol}^{-1}$ ) oxidative addition. From these considerations, first-row catalysts seem generally less suitable to engage in hydrogenation cycles that proceed through $\mathrm{H}_{2}$ oxidative addition (Scheme 1).

Ionic hydrogenation that relies on $\mathrm{H}_{2}$ heterolysis provides an alternative mechanistic scenario. ${ }^{81} \mathrm{H}_{2}$ activation and transfer along such a pathway is thermochemically defined by $\mathrm{M}-\mathrm{H}$ acidities $\left(\mathrm{p} K_{\mathrm{a}}\right)$ and hydricities $\left(\Delta G_{\mathrm{H}^{-}}\right)$. The most comprehensive body of experimental $\mathrm{p} K_{\mathrm{a}}$ data for metal hydride and dihydrogen complexes was compiled for group 8 compounds, yet with limited examples for full $\mathrm{Fe} / \mathrm{Ru} / \mathrm{Os}$-series in identical coordination environment. No clear trend along the group could be found, but the $\mathrm{p} K_{\mathrm{a}}$ differences between iron and ruthenium are generally small within 1-2 units while osmium compounds are less acidic accounting for stronger $5 \mathrm{~d} \mathrm{M}-\mathrm{H}$ bonding. ${ }^{67}$ In fact, Morris' increment methods for estimating $\mathrm{M}-\mathrm{H}$ acidities does not discriminate between first- and second-row metals. ${ }^{82}$

$\mathrm{M}-\mathrm{H}$ hydricities have a strong dependence on the metal and ligand sphere. Within a homologous $\mathrm{M}^{\mathrm{II}} \mathrm{H}(\text { diphosphine })_{2}{ }^{+}$ group 10 series, the nickel compound exhibited considerably higher hydricity in the order $\Delta G_{\mathrm{H}-}(\mathrm{Ni})>>\Delta G_{\mathrm{H}}-(\mathrm{Pt})>\Delta G_{\mathrm{H}-}$ (Pd) by more than $40 \mathrm{kcal} \mathrm{mol}^{-1}$, which renders nickel(II) by far the weakest hydride donor. ${ }^{83}$ The same general trend is observed for example for $\mathrm{M}^{\mathrm{III}} \mathrm{H}_{2}$ (diphosphine) ${ }^{+}$and $\mathrm{M}^{\mathrm{I}} \mathrm{H}$ (diphosphine) 2 ( $\left.\mathrm{M}=\mathrm{Co}, \mathrm{Rh}\right),{ }^{84-87}$ or computed hydricity values for $\mathrm{MH}(\mathrm{CO})_{5}\left(\mathrm{M}=\mathrm{Mn}, \mathrm{Re} ; \Delta \Delta G_{\mathrm{H}^{-}}=11\right.$ kcal mol $\left.{ }^{-1}\right) .{ }^{88} \mathrm{Kubiak}$ and co-workers recently showed for a broad range of $3-5 d$ transition metal hydrides that the hydridicities $\left(\Delta \Delta G_{\mathrm{H}^{-}} \approx 45 \mathrm{kcal} \mathrm{mol}^{-1}\right)$ exhibit a decent linear correlation with the one-electron reduction of the metal complexes in their respective hydride acceptor form $\left(E^{\mathrm{o}}\left([\mathrm{M}] /[\mathrm{M}]^{-}\right)\right) .{ }^{89}$ From a thermochemical cycle, hydricity $\Delta G^{\mathrm{o}} \mathrm{H}^{-}$can be expressed as a function of $\mathrm{M}-\mathrm{H}$ BDFE and the free energies for reduction of the complex $E^{\circ}\left([\mathrm{M}] /[\mathrm{M}]^{-}\right)$and the free hydrogen atom $\left(\Delta G^{\mathrm{o}}{\mathrm{H} / \mathrm{H}^{-}}^{-}\right)$:

$\Delta G_{\mathrm{H}^{-}}^{\mathrm{o}}=\mathrm{BDFE}_{\mathrm{M}-\mathrm{H}}+\mathrm{nF} E^{\mathrm{o}}\left([\mathrm{M}] /[\mathrm{M}]^{-}\right)+\Delta G^{\mathrm{o}}{\mathrm{H} / \mathrm{H}^{-}}^{-}$

The approximately linear correlation suggests that the variation in BDFE is comparatively small vs. the impact of the redox potential on hydricity. Strong hydride donor species will therefore be obtained for low complex charges and metal oxidation states and strongly $\sigma$-donating ligands (hydrides, alkylphosphines). Such ligands in labilizing transposition to the hydride will further reduce the M-H BDFE and therefore increase the hydricity. Corresponding trends were found for $\mathrm{Ru}$ ketone hydrogenation catalysts. ${ }^{90,91}$ From these consideration, strong-field pincer ligands seem to be a good choice due to the strong $\sigma$-donating ability. Furthermore, the stereochemical rigidity of pincer ligands enables to direct other strong-field ligands in trans-position to the catalytically relevant site. For example, a trans-dihydride configuration is generally found for active iron pincer catalyst species in de-/hydrogenation of polar substrates, which is discussed in section 3 .

The development of hydrogenation catalysts for polar multiple bonds and particularly the conceptual transfer to firstrow metal catalysis were strongly driven by the introduction of functional ligands that can serve as proton relays to facilitate $\mathrm{H}_{2}$ heterolysis (Scheme 2). An early, classical example in precious metal catalysis is Shvo's catalyst which carries a proton responsive cyclopentadienone ligand. ${ }^{92}$ This structural and functional motif could be transferred to iron catalysis by Knölker. ${ }^{4}$ Seminal contributions were made by Noyori's group reporting bisphoshine ruthenium catalysts with primary diamine ligands that exhibited extraordinary activities, chemoselectivities for $\mathrm{C}=\mathrm{O}$ over $\mathrm{C}=\mathrm{C}$ (transfer) hydrogenation and stereoselectivities. ${ }^{93,94}$ An 'NH-effect' 
was confirmed by a dramatic drop in activity in the absence of $\mathrm{N}-\mathrm{H}$ groups. It was originally attributed to cooperative $\mathrm{H}_{2}$ cleavage by the metal and a deprotonated amide ligand leading to an amine hydride complex with syn-configuration. Concerted hydride and proton transfer to the substrate without pre-coordination to the metal center was proposed as next step, to account for the high chemoselectivities and stereoinduction. ${ }^{93,95-101}$ However, stepwise outer-sphere mechanisms were more recently discussed as alternative scenarios challenging the direct involvement of an amide intermediate in catalysis. ${ }^{102}$

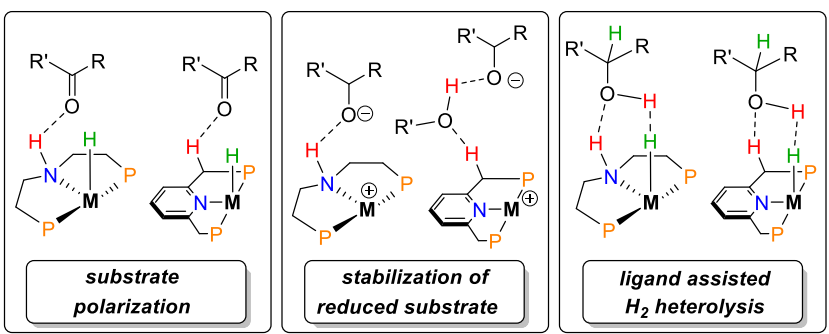

Figure 2. Exemplary outer-sphere processes of cooperating pincer ligands in de/hydrogenation catalysis. ${ }^{103}$

Several terms are used to describe the involvement of the auxiliary ligands in these reductive transformations. For Noyori's amine catalysts and systems inspired by them, $b i$ functional catalysis and Grützmacher's definition of metalligand cooperativity (MLC) are commonly applied, which requires ligands that actively participate in substrate activation and facilitate catalysis with the metal ion in a synergistic way. ${ }^{104}$ Within a more narrow definition metal and ligand undergo reversible chemical modifications in this process, such as ligand deprotonation/ reprotonation. ${ }^{105,106} \mathrm{As}$ pointed out by Dub and Gordon, cooperating ligands can also contribute to rate acceleration in ionic hydrogenation cycles without synergistic metal/ligand substrate activation, such as substrate polarization or outer-sphere stabilization of reduced substrate anions via hydrogen bonding (Figure 2). ${ }^{103}$ They therefore propose to reserve the term bifunctional catalysis for synergistic metal/ligand scenarios. We will here apply Milstein's widespread definition for MLC-assisted mechanisms, ${ }^{106}$ which includes reversible chemical steps at the ligand to distinguish them from alternative pathways that are often compared from computational evaluation. However, we want to emphasize the argument of Dub and Gordon that the role of these ligands in catalysis can be subtle and often offer different catalytic pathways that are close in energy.
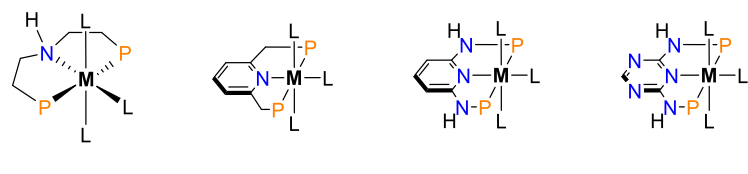

$P N^{H} P$

PN $^{\text {PyP }}$

$\mathrm{PN}_{3} \mathrm{P}$

$\mathrm{PN}_{5} \mathrm{P}$

Figure 3. Privileged metal-ligand cooperating pincer platforms in first-row de-/hydrogenation catalysis.
Over the last two decades several pincer platforms were introduced with excellent performance as privileged cooperating ligands in de/hydrogenation catalysis (Figure 3). ${ }^{106}$ The aliphatic $\mathbf{P N} \mathbf{N}^{\mathbf{H}} \mathbf{P}$ 'aminopincer' framework resembles structural features of Noyori's original catalysts and was also extensively utilized in first-row metal catalyzed de-/hydrogenation. In comparison to the pyridine- and triazine-based privileged ligands, the ligand backbone exhibits a high degree of flexibility, contrary to the original definition of pincer ligand.

Scheme 3. Proposed, MLC-assisted pathway for iron catalyzed ketone hydrogenation. ${ }^{107}$

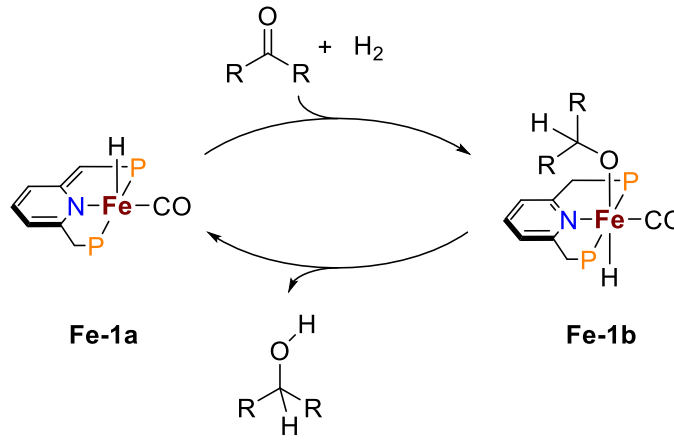

A major advance in the field of MLC-assisted catalysis was made by the Milstein group upon introduction of pyridinebased pincer ligand $\mathbf{P N}^{\mathbf{P y}} \mathbf{P}$ (Figure 3). ${ }^{51,107,109,110}$ Versatile synthetic access to this ligand platform strongly contributed to its widespread use. Deprotonation of a methylene group in the backbone produces an anionic, 'dearomatized' pincer ligand with amide character. Such dearomatized complexes can undergo stoichiometric, cooperative $\mathrm{H}_{2}$ heterolysis by 1,3-addition across the metal-ligand framework. This de/aromatization reactivity is often anticipated to be relevant for catalysis (Scheme 3). However, non-MLC-assisted pathways are also frequently encountered in de-/hydrogenation catalysis as discussed in the respective sections. Kirchner's $\mathbf{P N}_{\mathbf{3}} \mathbf{P}$ and the triazine-based $\mathbf{P N}_{\mathbf{5}} \mathbf{P}$ pincer ligands (Figure 3) represent variants with more acidic and rigid secondary amine groups in the backbone. ${ }^{110,111}$

Scheme 4. $\mathrm{H}_{2}$ 1,6-addition across a quinone-based PCP pincer iron(II) complex $\mathbf{F e - 2 a}$ and use for alkyne semihydrogenation $(\mathrm{P}=$ ${\left.\mathrm{P} i \mathrm{Pr}_{2}\right) .}^{112}$

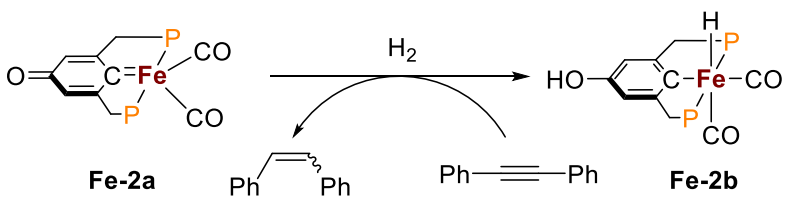

The application of these MLC-ligands had a tremendous effect on the advancement of first-row metal catalysis, particularly with $\mathrm{Mn}, \mathrm{Fe}$ and Co. ${ }^{20,21,25,26,30,46,113,114}$ Related pincer scaffolds were introduced that rely on alternative modes of ligand cooperativity. For example, a redox-active, carbenoid metallaquinone pincer ligand was reported, which undergoes long-range metal-ligand $\mathrm{H}_{2}$ 1,6-addition to form a ferrahydrochinone (Scheme 4). ${ }^{112}$ The reversible process 
allows for stoichiometric hydrogenation of alkynes and the compound was used for alcohol oxidation to aldehydes. ${ }^{12}$

Scheme 5. Stoichiometric, MLC-assisted $\mathrm{H}_{2}$-activation facilitated by functional PPP and PCP pincer ligands. ${ }^{15-119}$

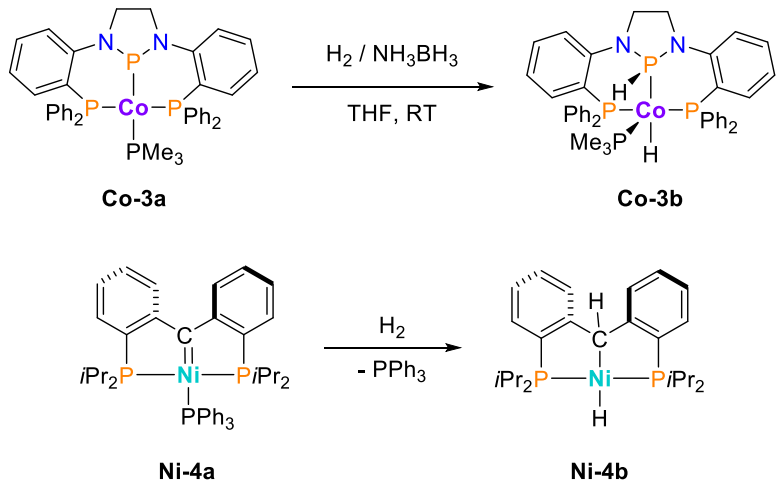

A rapidly growing structural and functional variety of cooperating pincer ligands with many different cooperating groups were subjected to stoichiometric bond activation reactions and some proved suitable for catalysis. For example, Thomas and coworkers described the 1,2-addition of dihydrogen across a covalent Co-P-bond (Scheme 5). ${ }^{115}$ Gutsulyak et al. reported 1,2-addition of E-H bonds $\left(\mathrm{E}=\mathrm{H}, \mathrm{NH}_{2}\right.$, $\left.\mathrm{OH}, \mathrm{OCH}_{3}, \mathrm{CCPh}, \mathrm{NHC}(\mathrm{O}) \mathrm{Ph}, \mathrm{SiPh}_{3}\right)$ at a nickel carbene PCP pincer complex, ${ }^{116-119}$ and Caulton and coworkers the 1,2-addition of dihydrogen along the Ni-N moiety of a cationic amido pincer Ni(II) complex (Scheme 6). ${ }^{120}$ The bimetallic Ni(I)/Ni(I) PBP pincer complex Ni-6a was reported by Peters to undergo reversible MLC-assisted addition of dihydrogen (Scheme 6) but is inactive in olefin hydrogenation. ${ }^{121}$

Scheme 6. Stoichiometric, MLC-assisted $\mathrm{H}_{2}$-activation facilitated by functional PNP and PBP pincer ligands. ${ }^{120,121}$

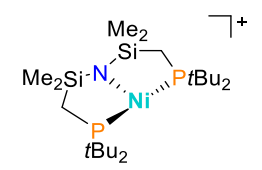

$\mathrm{Ni}-5 \mathrm{a}$
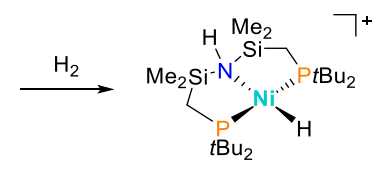

$\mathrm{Ni}-5 \mathbf{b}$

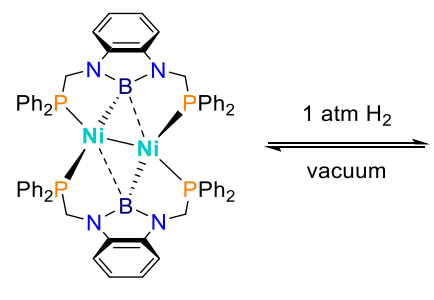

$\mathrm{Ni}-6 \mathrm{a}$

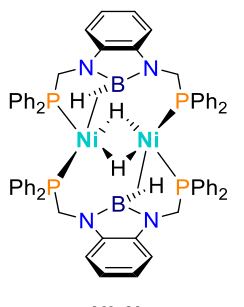

Ni-6b

\subsection{Redox Active Ligands}

The coordination and organometallic chemistry of $3 d$ vs. $4 / 5 \mathrm{~d}$ metals exhibits some critical differences with respect to (electronic) structure that strongly impact catalysis. $^{15,70,104,122-127}$ For example, coordinatively unsaturated iron complexes are much more frequent than for ruthenium owing to the stability of tetrahedral high-spin iron(II) in weak ligand fields. ${ }^{65}$ However, substrate binding to tetrahedral h.s.-Fe ${ }^{\mathrm{II}}$ requires considerable conformational rearrangement, which imposes an energetic penalty. Furthermore, spin pairing is usually a prerequisite to sufficiently activate a weakly binding substrate like $\mathrm{H}_{2}$. For example, transient ${ }^{3} \mathrm{Fe}(\mathrm{CO})_{4}$ exhibits drastically lowered rates towards $\mathrm{H}_{2}$ oxidative addition in comparison with ${ }^{1} \mathrm{Ru}(\mathrm{CO})_{4} \cdot{ }^{128,129}$ For iron, the ('spin-forbidden') reaction proceeds on two different spin surfaces. Spin-orbit coupling mixes the pure spin states in the region of surface crossing allowing for transition. However, in case of weak spin-orbit coupling $\left(\zeta\left(\mathrm{Fe}^{3+}\right)\right.$ $\left.=460 \mathrm{~cm}^{-1}, \zeta\left(\mathrm{Ru}^{3+}\right) \approx 1250 \mathrm{~cm}^{-1}, \zeta\left(\mathrm{Os}^{3+}\right) \approx 3000 \mathrm{~cm}^{-1}\right)^{130}$ and strong distortion in the crossing region, an additional kinetic barrier with low transmission probability may arise. ${ }^{131,132}$ The implications of these aspects on substrate binding are further exemplified by the diffusion dynamics of the 16-electron species ${ }^{3} \mathrm{Fe}(\mathrm{CO})_{4}$ vs. ${ }^{1} \mathrm{Cr}(\mathrm{CO})_{5}$ in hydrocarbon solvents, which is considerably slower for the singlet compound due to alkane coordination. ${ }^{133}$ From these considerations, rigid strong-field ligands, such as diphosphino pincers, that avoid trapping of active first-row catalysts in unproductive high-spin states should be beneficial for hydrogenation catalysis. ${ }^{15,54,70,122-124}$ However, redox-active weakfield pincer ligands (see below) provide an alternative approach. ${ }^{126}$ Notably, open-shell first-row catalysts proved instrumental in $\mathrm{C}-\mathrm{C}$ coupling ${ }^{125}$ and multi-state reactivity that crosses through low-lying spin surfaces along the reaction coordinate can accelerate catalytic transformations, which further complicates predictions. ${ }^{134,135}$

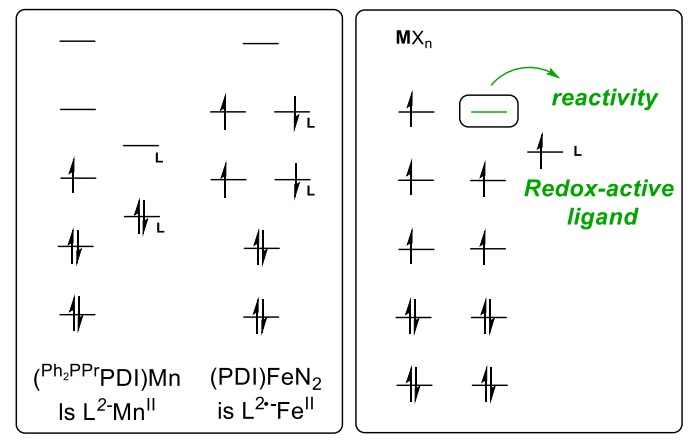

Figure 4. Schematic electronic configurations of $\left({ }^{\mathbf{P h}_{2} \mathbf{P P r}}{ }^{\mathbf{P D I}}\right) \mathrm{Mn}^{136}$ and (PDI)FeN ${ }_{2}{ }^{137}$

Redox-active ligands exhibit low lying electron donor or acceptor levels that result in distinctly ligand based redox events upon oxidation or reduction of respective metal complexes (Scheme 2). Ambiguous oxidation state assignments can arise in case of close energetic proximity of ligand and metal based frontier orbitals. ${ }^{137,138}$ Jørgensen coined the term redox non-innocence for cases where an accurate electron count within the metal valence shell is not possible. ${ }^{139}$ The terms redox-activity and (non-)innocence are frequently used in the same context and sometimes even synonymously, but should be distinguished to avoid confusion. ${ }^{127,140}$ For example, ligand non-innocence not necessarily refers to ligand-based radicals as implied by redox activity. Oxidation state ambiguity can also occur in closed shell systems, such as $(\mathbf{P D I}) \mathrm{Fe}(\mathrm{CO})_{2}$ or $(\mathbf{P D I}) \mathrm{Fe}\left(\mathrm{N}_{2}\right)_{2}(\mathrm{PDI}$ $=$ pyridinediimine ligand $)$ which can be described as $\left\{\left(\mathbf{P D I}{ }^{2-}\right.\right.$ 
) $\left.\mathrm{Fe}^{\mathrm{II}}\right\}$ or $\left\{(\mathbf{P D I}) \mathrm{Fe}^{0}\right\} .{ }^{137,141-143}$ The recent advances in modern spectroscopic tools and quantum-chemical electronic structure methods have strongly contributed to enable precise redox state assignments based on physical observables.

First-row transition metal ions with weak field ligands have a higher tendency to adopt high or intermediate spin states, such as $\left({ }^{\mathbf{P h}_{2} \mathbf{P P r} P D I}\right) \mathrm{MnCl}_{2},{ }^{136} \quad$ (PDI)FeCl $2,{ }^{144}$ and (PDI) $\mathrm{CoCl}_{2},{ }^{145}$ which is discussed in Section 2.2.1. In these cases, the high-spin $\mathrm{M}^{\mathrm{II}}$ ions are bound to neutral $\mathbf{P D I}{ }^{0}$ ligands. No direct involvement of these species in catalytic transformations have been reported, which can be attributed to the absence of vacant metal orbitals for substrate binding and activation. In contrast, the reduced complexes $\left(\mathbf{P D I}^{2-}\right.$ ) $\mathrm{Mn}^{\mathrm{II}}$, (PDI $\left.{ }^{2-}\right) \mathrm{Fe}^{\mathrm{II}} \mathrm{N}_{2}(\mathbf{F e}-7 \mathbf{a})$ and $\left(\mathbf{P D I}^{-}\right) \mathrm{Co}^{\mathrm{II}} \mathrm{Me}$ exhibit singly and doubly reduced PDI ligands bound to low spin $\mathrm{Mn}^{\mathrm{II}} / \mathrm{Co}^{\mathrm{II}}$ and intermediate spin $\mathrm{Fe}^{\mathrm{II}}$, respectively (Figure 4, left). Importantly, in these electronic configurations, vacant metal orbitals enable the binding and activation of substrates. For example, the iron complex undergoes oxidative addition of biphenylene (Scheme 7). The resulting five-coordinate ferric complex implies that the electron flow is both metal and ligand centered. ${ }^{146}$ Such active participation of the PDI ligand in oxidative addition ${ }^{147-151}$ and other redox reactions ${ }^{141,152-160}$ was well studied and this $\left(\mathbf{P D I}^{2-}\right) \mathrm{Fe}^{\mathrm{II}} /$ $\left(\mathbf{P D I}^{-}\right) \mathrm{Fe}^{\mathrm{III}}$ couple is the basis for many catalytic cyclization reactions. ${ }^{161}$ Similarly, $\left({ }^{\mathbf{P h}_{2} \mathbf{P P r}}{ }^{\mathbf{P D I}}\right) \mathrm{Mn}$ (Mn-8a) reacts with trifluoroacetophenone to a ketyl radical complex (Scheme 7). ${ }^{136}$ In this case, electron transfer is a purely ligand centered process. This manganese pincer platform has been utilized in catalytic hydrosilylation of carbonyl compounds.

Scheme 7. Ligand-directed reactions with small molecules. ${ }^{136,146,162}$

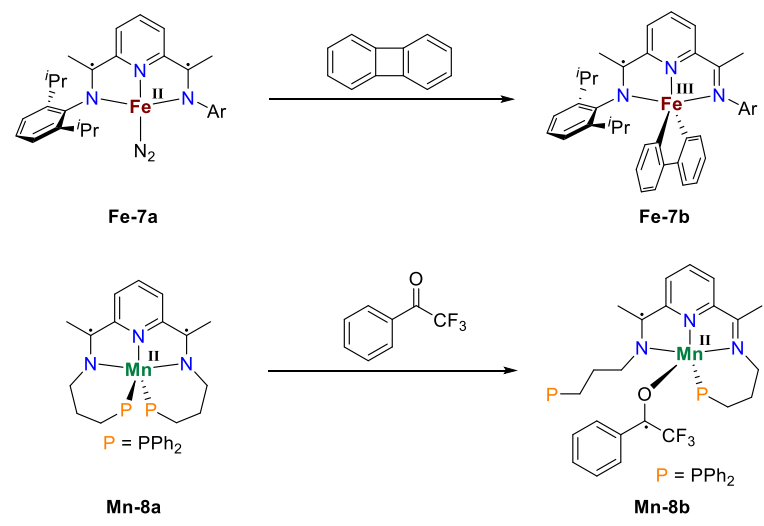

This exemplary reactivity can be attributed to two features (Figure 4, right): ${ }^{127,163}$ High lying, filled orbitals of the reduced pincer ligands serve as reservoir for reducing equivalents allowing the metal complexes to undergo (partially) ligand-based substrate reduction, such as oxidative addition, which is highly unusual for first-row metal ions coordinated by weak-field ligands. ${ }^{55,164,165}$ Furthermore, the Lewis acidity of the metal ions is enhanced by electron transfer to the redox-active pincer ligand and metal low- and intermediatespin states, thereby offering substrate binding sites. In consequence, strongly reducing metal-pincer platforms result, yet with empty metal-based orbitals for substrate binding reminiscent of low-spin complexes of low-valent, heavier transition metals stabilized by strong field ligands that are key species in catalysis. ${ }^{15,55,70,71,127,166}$

Besides the role as electron reservoir, ligand centered radical reactivity that leads to the formation of chemical bonds at the pincer backbone is frequently encountered and is associated with the term chemical-redox non innocence (Scheme 2). ${ }^{167-173}$ In the context of de-/hydrogenation catalysis pincer ligand centered, reversible proton coupled electron transfer seems most relevant combining both concepts of metal-ligand cooperativity and ligand redox activity. While applications in catalysis are much less frequent, an example was given by Goswami and co-workers (Scheme 8). ${ }^{174,175}$ The pyridine-based diazo pincer ligand undergoes stoichiometric, reversible hydrogen atom transfer with the azo/hydrazo couple. This reactivity allowed for application in catalytic aerobic alcohol oxidation. Within the proposed mechanism, the metal serves as template and has no active role with respect to bond formation/cleavage or redox events.

Scheme 8. Azo/hydrazo couple for alcohol dehydrogenation. ${ }^{174,175}$

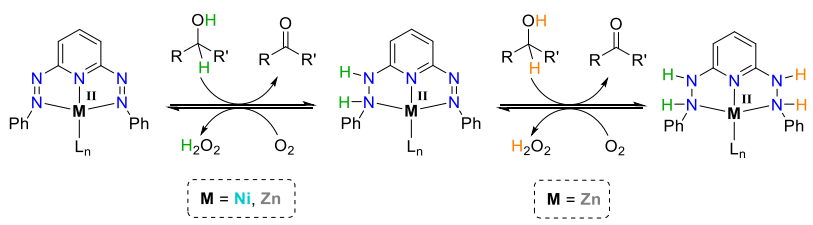

The bis(iminopyridyl) ligand (PDI), the bis(oxazolinepyridyl) ligand (Pybox) and the 2,2':6',2',-terpyridine ligand (terpy) are among the most frequently utilized, privileged, potentially redox active pincer ligands. All three ligand classes consist of a central pyridyl unit decorated with imine, pyridyl or oxazoline groups in 2-position, respectively. As a consequence of the rigid character, a meridional 'pincer-type' binding mode is generally favored for all three ligands. The weak-field character predominantly induces high-spin states unless other strong-field ligands are part of the coordination sphere or pincer redox-activity stabilizes lower spin-states. Particularly the PDI ligand exhibit distinct redox-active character and can accept up to three electrons, ${ }^{176,177}$ enabling the stabilization of an extended range of redox states and unusual coordination modes. ${ }^{144,178,179}$ The redox properties and coordination chemistry of these ligands with first-row metals will be presented in section 2.2 and applications in de-/hydrogenation catalysis in sections 3-5.

\section{PRIVILEGED PINCER PLATFORMS}

\subsection{Aminopincer Ligands}

\subsubsection{Diphosphinoamines and Related Ligands}

The aliphatic diphosphinoamine ligand family $\mathrm{HN}\left(\mathrm{CH}_{2} \mathrm{CH}_{2} \mathrm{PR}_{2}\right)_{2}\left({ }^{\mathbf{R}} \mathbf{P N}{ }^{\mathbf{H}} \mathbf{P}\right)$ was extensively utilized in $3 \mathrm{~d}$ metal catalysis, particularly for de-/hydrogenation reactions. The popularity can be ascribed to some key features:

1) The secondary amine functional group is acidified upon coordination to the Lewis acidic metal. The amide conjugate 
base facilitates heterolytic 1,2-addition of $\mathrm{H}_{2}$ across the $\mathrm{M}$ $\mathrm{N}$ bond. The active amide and amine hydride catalyst states are usually generated in situ (Scheme 9). However, the iron amide catalyst ( $\left.{ }^{\text {i r P }} \mathbf{P N P}\right) \mathrm{FeH}(\mathrm{CO})$ can be isolated, while the corresponding dihydride amine complex $\left({ }^{i \mathbf{P r}} \mathbf{P}\right.$ $\left.\mathbf{N}^{\mathrm{H}} \mathbf{P}\right) \mathrm{FeH}_{2}(\mathrm{CO})$ is only stable under $\mathrm{H}_{2}{ }^{180-182}$ In comparison, the manganese catalysts $\left({ }^{\mathbf{P r}} \mathbf{P N}{ }^{\mathrm{H}} \mathbf{P}\right) \mathrm{MnH}(\mathrm{CO})_{2}$ and

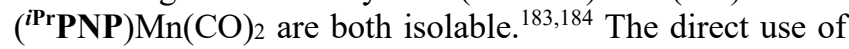
these active catalyst species enables catalysis under base free conditions, which can be beneficial to avoid side reactions such as base-catalyzed substrate aldol condensation. In some cases, borohydride precursors, such as (iPrP$\left.\mathbf{N}^{\mathrm{H}} \mathbf{P}\right) \mathrm{Fe}\left(\mathrm{BH}_{4}\right) \mathrm{H}(\mathrm{CO})$, can be used as a masked hydride that release $\mathrm{BH}_{3}$ with weak bases or thermally. ${ }^{10}$ Langer and coworkers found catalyst deactivation pathways by borane coordination to the pincer nitrogen donor. ${ }^{185}$ Related deactivation products were also found in ammonia-borane dehydrocoupling. ${ }^{186,187}$

Scheme 9. Generalized proposed outer-sphere hydrogenation mechanism for $\mathrm{d}^{6}{ }^{\mathrm{R}} \mathbf{P N}{ }^{\mathrm{H}} \mathbf{P}$ pincer catalysts.

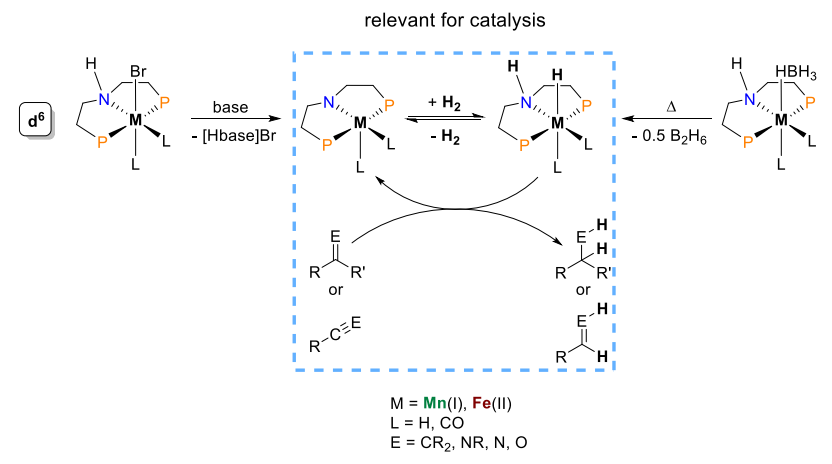

Despite the importance of reversible pincer backbone protonation for catalysis, experimentally derived $\mathrm{p} K_{\mathrm{a}}$ values of ${ }^{\mathrm{R}} \mathrm{PN}^{\mathrm{H}} \mathrm{P}$ amine complexes are surprisingly scarce. Schneider and co-workers reported $\mathrm{p} K_{\mathrm{a}}$ values in dmso-d $\mathrm{d}_{6}$ for the iridium(I) series $\left({ }^{\mathbf{i} P r} \mathbf{P N}{ }^{\mathbf{H}} \mathbf{P}\right) \mathrm{IrL}^{+}\left(\mathrm{L}=\mathrm{PMe}_{3}\right.$, cyclooctene, $\mathrm{CO}$; Scheme 10). ${ }^{188}$ The results demonstrate the tunability of the $\mathrm{N}-\mathrm{H} \mathrm{p} K_{\mathrm{a}}$ by the ligand environment and the strong acidification with respect to free dialkylamines (pyrrolidine: $\left.\mathrm{p} K_{\mathrm{a}}{ }^{\mathrm{dmso}} \approx 44\right) .{ }^{189}$ The $\mathrm{p} K_{\mathrm{a}}$ values of the iridium amine complexes are much closer to the dialkylammonium conjugate acids (pyrrolidinium: $\left.\mathrm{p} K_{\mathrm{a}}^{\mathrm{dmso}}=11.1\right) .{ }^{190}$ Notably, the $\mathrm{MN}$ $\mathrm{H} \mathrm{p} K_{\mathrm{a}}$ is in the range reported for some iron dihydrogen and hydride complexes. ${ }^{82,191}$

Scheme 10. $\mathrm{p} K_{\mathrm{a}}$ determination for $\operatorname{Ir}\left({ }^{i{ }^{P r}} \mathbf{P N}{ }^{\mathrm{H}} \mathbf{P}\right)$ complexes. ${ }^{188}$

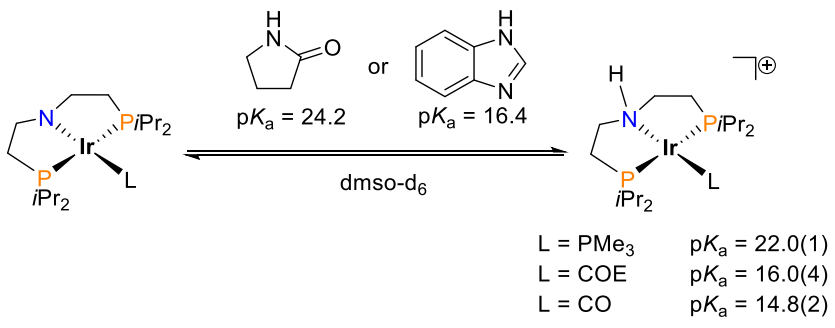

2) The strong-field phosphine ligands stabilize active catalyst species in low-spin states avoiding unproductive radical chemistry. Iron and manganese catalysts generally exhibit $\mathrm{d}^{6}$ configuration. In contrast to analogous heavier $4 \mathrm{~d}$ and $5 \mathrm{~d}$ catalysts, additional $\pi$-accepting strong field ligands are required for first row metals to maintain low-spin configuration, which so far limits the selection of neutral auxiliary ligands for $\mathrm{Fe}$ and $\mathrm{Mn}$ to $\mathrm{CO}$ and isonitriles. ${ }^{192}$ For earlier $3 \mathrm{~d}$ metals, isoelectronic formal V(-I) $\left(\left({ }^{i \mathbf{P r}} \mathbf{P N P}\right) \mathrm{V}(\mathrm{NO})_{2} /\left({ }^{i \mathbf{P r}} \mathbf{P}-\right.\right.$ $\left.\left.\mathbf{N}^{\mathbf{H}} \mathbf{P}\right) \mathrm{VH}(\mathrm{NO})_{2}\right)$ and $\mathrm{Cr}(0)\left(\left({ }^{i \mathbf{P r}} \mathbf{P N P}\right) \mathrm{Cr}(\mathrm{NO})(\mathrm{CO}) /\left({ }^{i \mathbf{P r}} \mathbf{P}-\right.\right.$ $\left.\mathbf{N}^{\mathrm{H}} \mathbf{P}\right) \mathrm{CrH}(\mathrm{NO})(\mathrm{CO})$ ) analogues of the $\mathrm{Fe}$ and $\mathrm{Mn}$ platforms were computationally evaluated. ${ }^{193,194}$ Both systems were pointed out as promising candidates for catalytic $\mathrm{C}=\mathrm{E}(\mathrm{E}=$ $\mathrm{C}, \mathrm{N}, \mathrm{O}$ ) hydrogenation waiting for experimental confirmation.

3) Within the octahedral geometry, the amine and hydride mutual cis-arrangement acts as a template for outer-sphere $\mathrm{H}^{+} / \mathrm{H}^{-}$transfer to the substrate. The strong-field trans-ligands, like hydride $(\mathrm{Fe})$ or $\mathrm{CO}(\mathrm{Mn})$ increase the hydricity of the transferred hydride and reduce the kinetic barrier of outer-sphere hydrogen transfer to the substrate..$^{68,91}$

4) The coordinatively and electronically unsaturated five-coordinate amide species that result from $\mathrm{H}^{+} / \mathrm{H}^{-}$transfer are stabilized by the strongly $\pi$-donating dialkyl amide ligand. The single-faced $\pi$-donor destabilizes a low-lying d-orbital. As a result, the $d^{6}\left(\mathrm{Fe}^{\mathrm{II}}, \mathrm{Mn}^{\mathrm{I}}\right)$ complexes preferentially adopt trigonal-bipyramidal geometries with Y-shaped distortion and electronic low-spin configuration..$^{52}$ Importantly, the accessible low-spin state is a prerequisite to subsequent $\mathrm{H}_{2}$ binding and activation (Scheme 11).

Scheme 11. Generalized proposed inner-sphere hydrogenation mechanism for $\mathrm{d}^{8} \mathbf{R} \mathbf{P N}{ }^{\mathrm{H}} \mathbf{P}$ pincer catalysts.

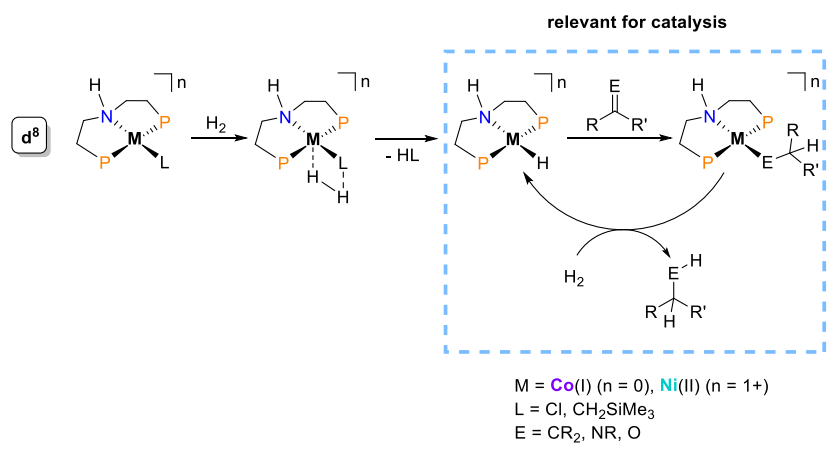

The cobalt pre-catalysts with this pincer ligand exhibit distinct structural differences with the $\mathrm{Mn}$ and $\mathrm{Fe}$ platforms. Hanson's catalyst starts from the square-planar, low-spin cobalt(II) precursor ( $\left.{ }^{\mathrm{Cy}} \mathbf{P N P}\right) \mathrm{Co}\left(\mathrm{CH}_{2} \mathrm{SiMe}_{3}\right)$ activated in situ by Brookhart's acid, which is discussed in Section 3.1.2. ${ }^{195}$ Notably, while the iron(II) catalysts show high chemoselectivity for polar multiple bonds, ${ }^{196}$ Hanson's catalyst also exhibits high activity for the hydrogenation of unactivated olefins. The different selectivities were attributed to an innersphere olefin insertion/ $\sigma$-metathesis cycle by an in-situ formed, square-planar, low-spin cobalt(II) hydride as discussed in Section 3.7.2. In contrast, computational treatment suggests that an MLC-assisted outer-sphere mechanism might be relevant for $\mathrm{C}=\mathrm{O}$ hydrogenation. ${ }^{197,198}$ Arnold and coworkers also described dinuclear $\mathrm{H}_{2}$ activation routes for 
the CoPNP system. ${ }^{199,200}$ The role of such pathways for catalysis has not been examined. The cobalt catalysts are in general mechanistically less well defined as their iron counterparts. Hanson and coworkers also utilized the aminopincer ligand for nickel(II) catalyzed olefin hydrogenation. ${ }^{201}$

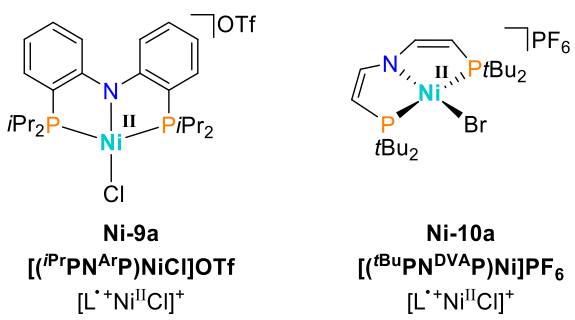

Figure 5. Redox-active PNP ligands. ${ }^{202,203}$

The Mindiola group reported that the oxidation of nickel(II) diphosphinoanilide complex $\left.\left[{ }^{i \mathbf{P r}} \mathbf{P N}{ }^{\mathrm{Ar}} \mathbf{P}\right) \mathrm{NiCl}\right] \mathrm{OTf}(\mathbf{N i - 9 a})$ $\left(E^{\mathrm{o}}=-0.06 \mathrm{~V}\right.$ vs. $\left.\mathrm{Fc} / \mathrm{Fc}^{+}\right)$is a mainly ligand centered process (Figure 5). ${ }^{202}$ Aliphatic analogues of this ligand can be obtained by oxidation of ( $\left.{ }^{(\mathrm{Bu} P N P}\right) \mathrm{M}$ complexes $(\mathrm{M}=\mathrm{Co}, \mathrm{Ni})$ with 2,4,6-tri-tert-butylphenoxy radical as hydrogen acceptor (Figure 5). ${ }^{203,204}$ As for the diphosphinoanilide nickel(II) complex, oxidation of the aliphatic bromide analogue at $0.19 \mathrm{~V}$ is mainly ligand centered. In addition, the aliphatic pincer nickel(II) complex can be protonated at a vinylene bridge $\left(\mathrm{p} K_{\mathrm{a}}^{\mathrm{dmso}}=0.9\right)$. In consequence, the oxidized complex $\mathrm{NiBr}\left\{\mathrm{N}(\mathrm{CHCHP} t \mathrm{Bu})_{2}\right\}^{+}(\mathbf{N i}-10 a)$ activates benzylic substrates by $\mathrm{C}-\mathrm{H}$ hydrogen atom transfer (HAT) with the pincer ligand serving as both electron and proton acceptor. ${ }^{203}$ Ligand-centered HAT was also reported by Heyduk and coworkers for a nickel complex with a phenylene-bridged, trianionic SNS pincer (Scheme 12). ${ }^{205}$ In that case the nitrogen atom serves as proton acceptor. HAT with such radical pincer ligands has not been utilized for catalysis to date.

Schneider and coworkers reported an abnormal $\mathrm{CO}_{2}$ insertion reaction for the nickel(II) hydride $\mathrm{NiH}\left\{\mathrm{N}\left(\mathrm{CHCHP} t \mathrm{Bu}_{2}\right)_{2}\right\}$ (Ni-10c) under photolytic conditions (Scheme 13) ${ }^{206}$ Based on transient spectroscopy, kinetic examinations and trapping experiments the reaction was attributed to a photochemically driven trans-NH reductive elimination and subsequent $\mathrm{CO}_{2}$ activation by nickel( 0 ), representing a photochemical example of metal-ligand-cooperativity. Subsequently, photo-driven reverse water-gasshift could be established within a two-step synthetic cycle (Scheme 13) ${ }^{207} \mathrm{H}_{2}$ heterolysis proceeds by proton transfer to a pincer backbone vinylene bridge emphasizing the relation as aliphatic analogues of the pyridine based PNP pincer ligands, which is discussed in Section 2.2.1.
Scheme 12. Thermochemical Cycles for the BDFE determination of C-H HAT (Ni-10b) and N-H HAT (Ni-11a) reactivity. ${ }^{203,205}$

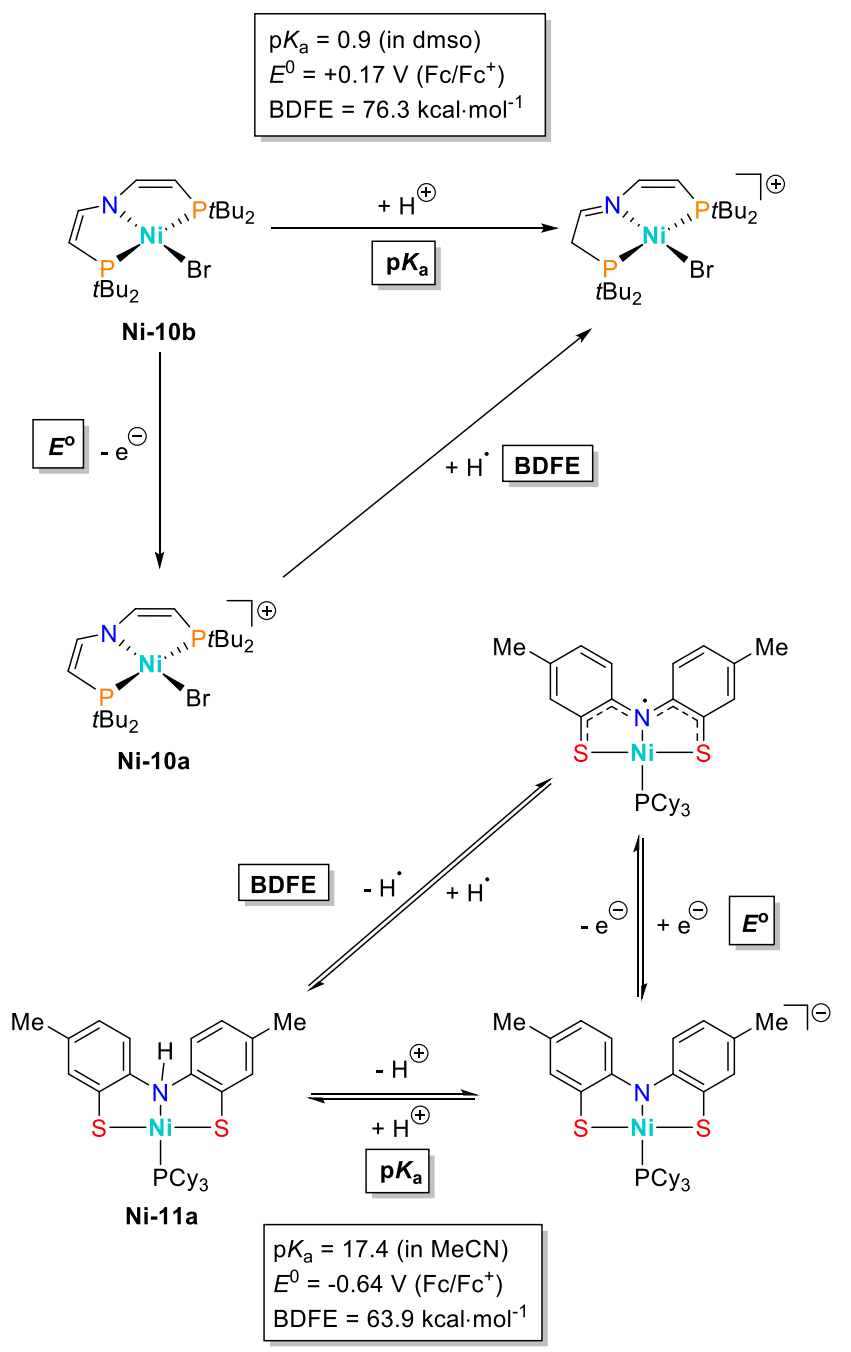

Scheme 13. Reactivity of Dehydrogenated Amido Pincer Ni(II) Complexes Towards $\mathrm{CO}_{2}$ and Two-Step Photochemically Driven Reverse Water-Gas Shift Chemical Cycle $\left(\mathrm{P}=\mathrm{P} t \mathrm{Bu}_{2}\right) .{ }^{206}$

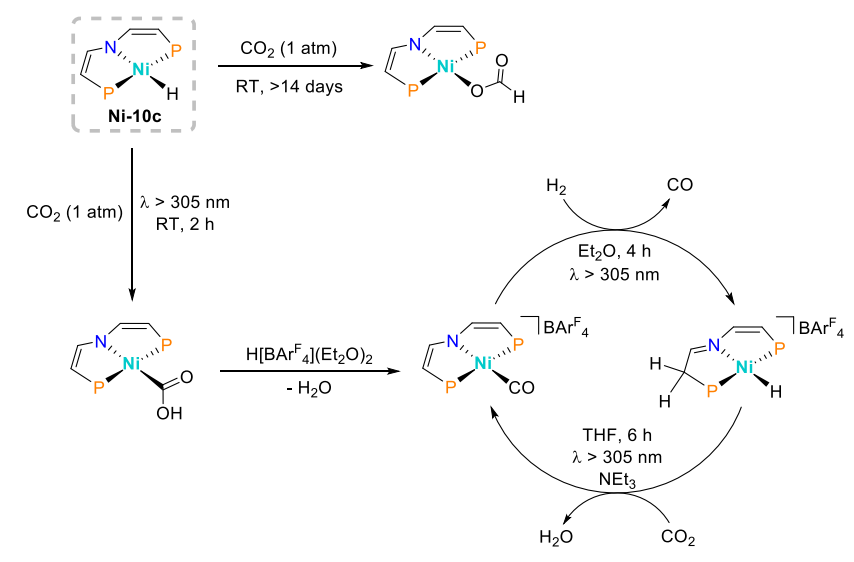

2.1.2. BPI and Boxmi Ligands Gade and co-workers reported a series of indolene based NNN-pincer ligands with different backbones (Figure 6). The spin density distribution in the four-coordinate, divalent iron complex with a 1,3bis(2-pyridylimino)isoindolines ligand was examined by 
means of ${ }^{13} \mathrm{C}$ NMR spectroscopy. ${ }^{208}$ Iron, cobalt and manganese complexes with this family of ligands were utilized in enantioselective ketone reduction, ${ }^{209}$ enantioselective ketone hydrosilylation, ${ }^{210,211}$ and enantioselective ketone hydroboration. ${ }^{212,213}$

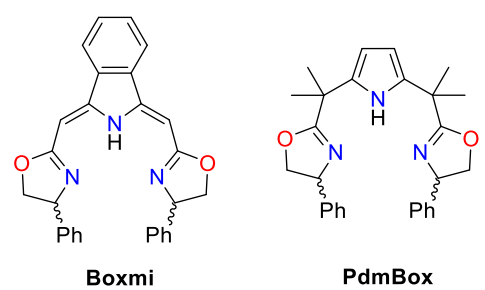

Figure 6. Boxmi and PdmBox ligands. ${ }^{214,215}$

2.1.3. Aminophenol Pincer Ligands Bulky phenols can form stable phenoxy radicals. ${ }^{216}$ Accordingly, phenolic substituents were used as building blocks in redox-active pincer ligands. ${ }^{217}$ The amino phenol $\mathrm{NNO}^{\mathrm{ISQ}}$ ligand used in $\left(\mathbf{N N O}^{I S Q}\right) \mathrm{FeCl}_{2}$ (Fe-12) was initially utilized for palladium mediated, intramolecular C-H amination. ${ }^{218}$ The Fe complex exhibits high-spin iron(III) antiferromagnetically coupled to a pincer radical anion. CV measurements showed one quasireversible reductive wave at $-0.74 \mathrm{~V}$ (vs. $\mathrm{Fc} / \mathrm{Fc}^{+}$) and one quasireversible oxidation at $0.51 \mathrm{~V}\left(\mathrm{vs} . \mathrm{Fc} / \mathrm{Fc}^{+}\right)$, which are both proposed to be ligand centered. This compound catalyzes intramolecular $\mathrm{C}\left(\mathrm{sp}^{3}\right)-\mathrm{H}$ amination. ${ }^{219}$ The $\mathrm{NNO}^{I S Q}$ ligand is also available with a tethered phosphine group, which was utilized for ligand centered disulfide activation. ${ }^{220}$

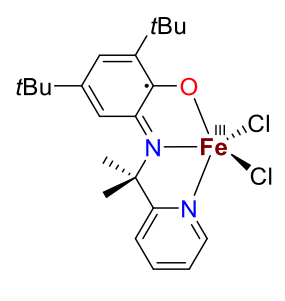

Fe-12 hs $\mathrm{L}^{\circ} \mathrm{F} \mathrm{Fe}^{\prime \prime \prime} \mathrm{Cl}_{2}$

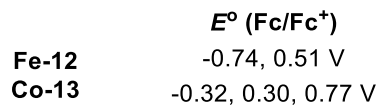

Figure 7. Redox-active aminophenol ligands. ${ }^{219,221}$

The cobalt complex ( $\left.{ }^{\boldsymbol{S}} \mathbf{O C O}\right) \mathrm{Co}($ thf $)(\mathbf{C o - 1 3})$ (Figure 7) was reported by Soper and co-workers as a low spin Co(II) species with a dianionic, closed-shell bisphenolate ligand. ${ }^{221}$ Three redox states were detected by $\mathrm{CV}$ as quasi reversible events at $-0.32,0.30$ and $0.77 \mathrm{~V}$, respectively, and proposed to be not exclusively metal centered. Chemical oxidation gave rise to the mono and dicationic species best described as cobalt(II) in both cases. ${ }^{221}$ Related amino-diamine ligands are redox-active but have a facial coordination motif and are thus no further discussed in this review. ${ }^{22}$

2.1.4. Dihydrazonopyrroles Anderson and co-workers recently reported the design of a novel dihydrazonopyrrol ligand (Figure 8). ${ }^{223}$ The ligand architecture represents a modification of known diiminopyrrole ligands to avoid undesired bidentate coordination modes upon introduction of sterically shielding substituents and an extended backbone.
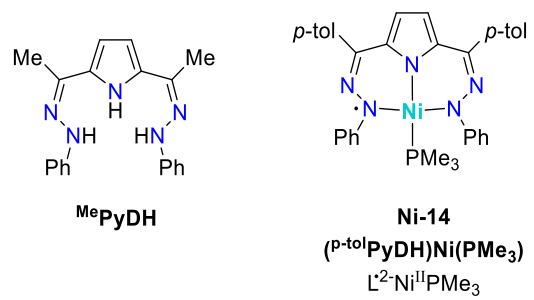

Figure 8. Dihydrazonopyrrol ligand and coordination with nickel. ${ }^{223}$

Coordination of nickel gave a square planar trimethylphosphine complex. EPR-Spectroscopy confirmed a ligand centered radical and computational analysis revealed the majority of the spin density on the ligand giving rise to formulation as divalent nickel in complex Ni-14. This complex is prone to oxidation and reduction both as ligand-centered processes. Furthermore, reversible hydrogen atom transfer was observed. ${ }^{223}$

\subsection{Pyridyl Pincer Ligands}

\subsubsection{Diphosphinopyridine and Related Lig-} ands The diphoshinopyridine ligands $\mathrm{NC}_{5} \mathrm{H}_{5}-2,6-$ $\left(\mathrm{CH}_{2} \mathrm{PR}_{2}\right)_{2} \quad\left({ }^{\mathbf{R}} \mathbf{P N} \mathbf{N}^{\mathbf{P y}} \mathbf{P}\right)$ represent an archetypal cooperative ligand class. $\mathrm{Fe}(\mathrm{II}), \mathrm{Co}(\mathrm{II})$ and $\mathrm{Ni}$ (II) metal complexes with $\mathbf{P N}^{\mathbf{P y}} \mathbf{P}$ ligands were first reported in 1971 by Nelson and coworkers. $^{224}$ The cyclic backbone results in more rigidity compared to the $\mathbf{P N} \mathbf{N}^{\mathbf{H}} \mathbf{P}$ ligand, but the pincer ligand arms still allow for some degree of conformational freedom. Milstein introduced a concept to catalysis that is referred to as "aromatization-dearomatization" as a distinctive feature of the ${ }^{\mathbf{R}} \mathbf{P N}{ }^{\text {Py}} \mathbf{P}$ platform. ${ }^{108,109,225}$ Several analogies and differences with respect to the aliphatic PNP are apparent:

1) The benzylic methyl protons of 2-methylpyridine exhibit a $\mathrm{p} K_{\mathrm{a}}$ in THF around $35 .{ }^{226}$ Accordingly, the free pincer ligand can be deprotonated once and even twice by strong bases like $\mathrm{KCH}_{2} \mathrm{Ph}\left(\mathrm{p} K_{\mathrm{a}}^{\mathrm{THF}} \approx 42\right)$ and $\mathrm{LiCH}_{2} \mathrm{SiMe}_{3}$, respectively, giving the corresponding alkali salts enabling the synthesis of "dearomatized" transition metal complexes via salt metathesis. ${ }^{27,228}$ However, more common access, e.g. to Re, ${ }^{229}$ $\mathrm{Ru},{ }^{230-232} \mathrm{Rh},{ }^{233} \mathrm{Ir}^{234,235} \mathrm{Ni}^{236} \mathrm{Pd}^{237,238}$ and $\mathrm{Pt}^{237-239}$ complexes, is provided by dehydrohalogenation after coordination of the neutral diphosphinopyridine ligand to transition metal ions. Benzylic deprotonation is stabilized by charge delocalization within the pyridyl core. The relevance of the amido resonance structure is evidenced by a shortened C-C bond in the pincer backbone, in analogy to the aliphatic enamido pincer ligand. ${ }^{204}$ Milstein and coworkers also demonstrated the two-fold benzylic deprotonation of a nickel(II) complex with methyllithium. ${ }^{236}$ Recently, the Ozerov group could isolate a dearomatized rhenium diphosphinophenyl complex. $^{240}$

2) The PNP ligands in combination with additional strong field ligands (hydride, $\mathrm{CO}$ ) stabilize $\mathrm{d}^{6}$ low-spin states of $3 \mathrm{~d}$ 
metals, which enables binding of substrates like $\mathrm{H}_{2}$. In comparison with the dialkylamido pincers, the weaker $\pi$-donation by the dearomatized ligands further increases metal Lewis acidity. For example, in contrast to $\left({ }^{t \mathbf{B u}} \mathbf{P N}{ }^{\mathrm{H}} \mathbf{P}\right) \mathrm{Fe}(\mathrm{H})_{2}(\mathrm{CO})$ (see above), the trans-dihydride complex $\left({ }^{t \mathbf{B u}} \mathbf{P N}{ }^{\mathbf{P y}} \mathbf{P}\right) \mathrm{Fe}(\mathrm{H})_{2}(\mathrm{CO})$ could be isolated. ${ }^{241}$ In turn, the dearomatized monohydride complex could only be isolated with an additional sixth ligand. ${ }^{107}$ Alternative access to octahedral, aromatized hydride catalyst species is provided by thermal activation of borohydride complexes, which allows for base-free catalysis.

3) The ligand-centered basicity of dearomatized $\mathbf{P} \mathbf{N}^{\mathbf{P y}} \mathbf{P}$ complexes enables the heterolysis of substrates like $\mathrm{H}_{2}$ with (re)aromatization of the pyridyl moiety and formation of a $\mathrm{M}-\mathrm{H}$ bond (Scheme 14). ${ }^{107}$ The 1,3-addition is a distinguishing feature with respect to the aliphatic amidopincer systems (1,2-addition).

Scheme 14. General Cooperativity Mechanism of the $\mathbf{P N} \mathbf{N}^{\mathbf{P y}} \mathbf{P}$ Pincer Complexes with $\mathrm{d}^{6}$ Electronic Configuration for Activation and Addition of Dihydrogen Across Different C-C-, C-N- and C-OMultiple Bonds.

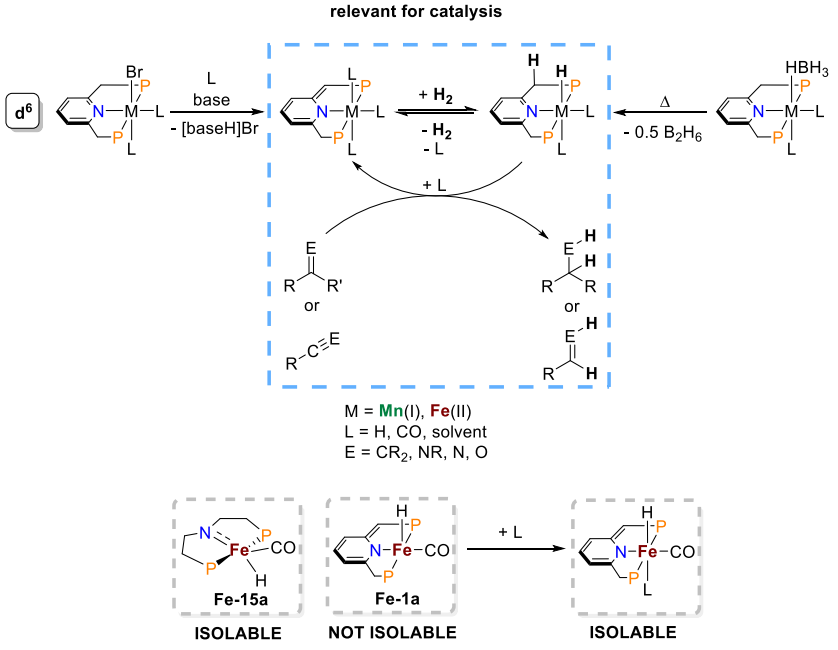

Regarding first row metals, particularly iron(II) and to a lesser extent manganese(I) complexes have been utilized for hydroelementation catalysis due to facile accessibility of $\mathrm{d}^{6}$ low-spin precatalysts. Metal-ligand cooperative reactivity for isoelectronic cobalt(III) complexes is not known to date. Milstein and coworkers reported the formal loss of a hydrogen atom at the $\mathrm{CH}_{2}$ linker of a $\mathrm{d}^{8}$ pyridyl pincer $\mathrm{Co}(\mathrm{I})$ methyl complex. ${ }^{242}$ Unlike the corresponding PDI complex (see below), the methyl complex ( $\left.{ }^{\left({ }^{B u}\right.}{ }^{P} N^{\mathbf{P y}} \mathbf{P}\right) \mathrm{CoMe}(\mathbf{C o - 1 a}$, Figure 9) features low-spin cobalt(I) as a consequence of the higher lying pincer acceptor levels. This compound undergoes hydrogen atom loss at one of the methylene bridges, and the resulting product was initially described as low-spin cobalt(I) bound to a radical pincer ligand. ${ }^{242}$ This assignment was later revised for cobalt(II) with a dearomatized, anionic pincer ligand $(\mathbf{C o - 1 b})$ based on EPR characterization and supporting DFT studis. ${ }^{243,244}$

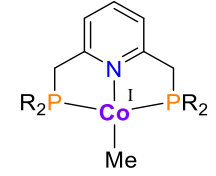

Is (PNP)Co'Me

closed shell $\mathrm{Co}(\mathrm{l})$

Co-1a

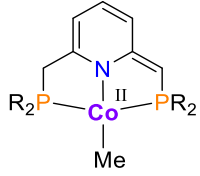

Is (PNP)Co"Me

metal centered radical

Co-1b
Figure 9. Comparison in the electronic structure between cobalt methyl PNP complexes. ${ }^{242,243}$

Van der Vlugt and coworkers reported the synthesis of dearomatized nickel(II) complexes and 1,3-addition of benzenethiol. ${ }^{245}$ The same group also explored the coordination chemistry and reactivity of copper(I) complexes with dearomatized ligands, ${ }^{246,247}$ providing evidence for metalligand cooperativity in copper catalyzed [2+3] cycloaddition of phenylacetylene and benzyl azide. ${ }^{248}$ Early transition metals complexes ( $\mathrm{Sc}, \mathrm{Ti}, \mathrm{V})$ with this ligand have not been reported and chromium complexes are rare. ${ }^{249}$

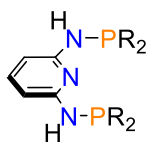

$\mathbf{R}_{\mathbf{P N}_{3}} \mathbf{P}$

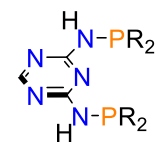

${ }^{\mathrm{R}} \mathrm{PN}_{5} \mathrm{P}$
Figure 10. $\mathrm{PN}_{3} \mathrm{P}$ and $\mathrm{PN}_{5} \mathrm{P}$ ligands.

In analogy to the parent $\mathbf{P N}{ }^{\mathbf{P y}} \mathbf{P}$ ligand, the pyridine aminophosphine ligands ${ }^{\mathbf{R}} \mathbf{P N}_{3} \mathbf{P}$ and the pyrimidine-based ${ }^{\mathbf{R}} \mathbf{P N}_{5} \mathbf{P}$ pincer ligand was extensively used in catalysis (Figure 10). Manganese, iron and cobalt catalysts with ${ }^{\mathrm{R}} \mathbf{P N}_{3} \mathbf{P}$ and ${ }^{\mathrm{R}} \mathbf{P} \mathbf{N}_{5} \mathbf{P}$ ligands were established by the groups of Kirchner and Kempe, respectively. ${ }^{113,250}$ The exocyclic NH-bridges of both $\mathbf{P N}_{3} \mathbf{P}$ and $\mathbf{P N}_{5} \mathbf{P}$ ligands are more acidic than the benzylic groups of the parent $\mathbf{P} \mathbf{N}^{\mathbf{P y}} \mathbf{P}$ ligand, giving rise to substrate activation by 1,3 -addition at dearomatized ligands. Heterolytic cleavage of dihydrogen was reported with an $\mathrm{Fe}\left(\mathbf{P N}_{3} \mathbf{P}\right)$-complex after reduction of one of the $\mathrm{NH}$ moieties with zinc powder. ${ }^{251}$

Nickel(II) $\mathbf{P N}_{3} \mathbf{P}$ and $\mathbf{P N}_{5} \mathbf{P}$ pincer complexes were utilized as catalysts for Suzuki-Miyaura, ${ }^{252}$ Kumada-Corriu and Negishi cross-coupling reactions. ${ }^{253}$ Copper(II) $\mathbf{P N}_{3} \mathbf{P}$ catalysts were utilized for Suzuki-Miyaura cross-coupling. ${ }^{254}$ Early transition metal complexes (Sc, Ti) were not reported. Vanadium and chromium complexes are scarce and chromium complexes were used as catalysts in olefin oligomerization. $^{255-257}$

\subsubsection{Pyridinediimines and Related Ligands}

The pyridinediimine (PDI) pincer family represents one of first privileged ligands that sparked the current developments in $3 \mathrm{~d}$ metal catalysis. After Brookhardt's and Gibson's landmark discoveries of the remarkable catalytic activity of iron and cobalt PDI complexes in ethylene polymerization, ${ }^{258-260}$ numerous studies have been conducted ${ }^{258,261-}$ ${ }^{281}$ and were thoroughly reviewed. ${ }^{282-286}$ The synthetic modularity and accessibility offer modifications that give rise to many applications of PDI ligands in 3d-metal catalyzed C- 
C coupling ${ }^{158,161,165-167,287-294}$ de-/hydrogenation, ${ }^{295-299}$ hydrosilylation, ${ }^{295,298,300,301}$ hydroboration, ${ }^{302}$ and electrochemical transformations. ${ }^{303}$ Its use in iron catalysis was inspired by isolobal relationship considerations of the $\mathrm{Fe}(\mathrm{CO})_{3}-$ fragment with (PDI)Fe. ${ }^{305-308}$ Low-lying PDI $\pi^{*}$-orbitals support electron-rich, active key species in catalysis.

Three key positions within the PDI platform have been systematically varied with significant effects on the redox properties and chemical reactivity, i.e. (a) the 4-position of the pyridine core, (b) the substitution pattern of the $\mathrm{C}_{\text {imine-atom, }}$ and (c) imine $N$-substitution (Figure 11). Free PDI ligands generally exhibit electrochemically reversible $\mathrm{PDI} / \mathrm{PDI}^{\circ-}$ redox couples below $-2.5 \mathrm{~V}$ (vs. $\mathrm{Fc} / \mathrm{Fc}^{+}$), such as the 'arche-

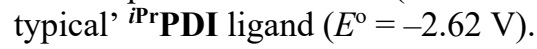

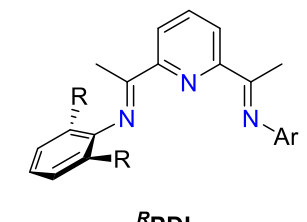

$R_{\text {PDI }}$
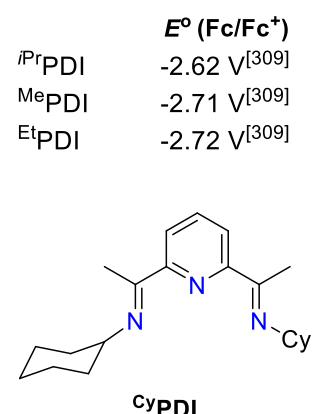

$E^{\circ}\left(\mathrm{Fc} / \mathrm{Fc}^{+}\right)$

${ }^{\mathrm{Cy}} \mathrm{APDI} \quad-2.83 \mathrm{~V}^{[309]}$

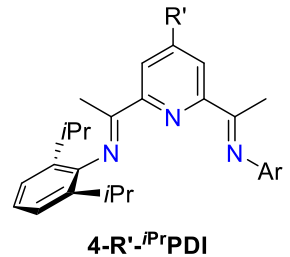

$E^{\circ}\left(\mathrm{Fc} / \mathrm{Fc}^{+}\right)$

4-Bn- ${ }^{i P r} \mathrm{PDI} \quad-2.72 \mathrm{~V}^{[308]}$

$4-\mathrm{NMe}_{2}{ }^{i \mathrm{Pr} P D I} \quad-2.82 \mathrm{~V}^{[308]}$

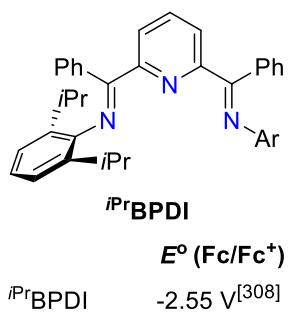

4- $\mathrm{CF}_{3^{-}}{ }^{i \mathrm{Pr} P D I} \quad-2.35 \mathrm{~V}^{[308]}$

Figure 11. PDI ligands and their redox potentials. ${ }^{308,309}$

Substitution in the pyridine para-position has a strong effect on the ligand acceptor orbitals and allows for precise fine tuning the reduction potential by almost $\Delta E^{\mathrm{o}}=0.5 \mathrm{~V} .^{308,309}$ Phenyl vs. methyl substitution at the $C$ imine position shifts the reduction potential anodically, yet less significantly, by about $0.1 \mathrm{~V},{ }^{309}$ enabling, e.g. the stabilization of an electron rich iron(I) dinitrogen complexes with a trianionic PDI ligand. However, phenyl substitution in this position can lead to irreversible catalyst deactivation pathways. ${ }^{152,158,295}$

The reduced radical ligand $\mathrm{Na}\left({ }^{i \mathbf{P r}} \mathbf{B P D I}\right)$ was characterized by X-band EPR spectroscopy at room temperature. ${ }^{374} \mathrm{~A}$ sharp, isotropic 10 line signal at $g_{\text {iso }}=2.003$ was observed. Simulations revealed hyperfine interactions (HFI) with the pyridine $\left(A_{\text {iso }}=6.2 \mathrm{MHz}\right)$ and imine $\left(A_{\text {iso }}=3.3 \mathrm{MHz}\right){ }^{14} \mathrm{~N}$ nuclei, the para-hydrogen atom $\left(A_{\text {iso }}=14.0 \mathrm{MHz}\right)$ and the ${ }^{23} \mathrm{Na}$ counter cation $\left(A_{\text {iso }}=8.4 \mathrm{MHz}\right)$. The EPR spectrum of the ${ }^{\text {iPr} P D I ~ r a d i c a l ~ a n i o n ~ i s ~ m o r e ~ c o m p l e x ~ p r e s u m a b l y ~ d u e ~ t o ~}$ additional HFI with the imine methyl groups.

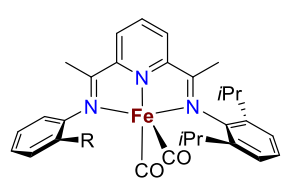

$\mathrm{Fe}-7 \mathrm{c}-\mathrm{Fe}-7 \mathrm{f}$

$\left({ }^{R, \mathrm{PP} P D I}\right) \mathrm{Fe}(\mathrm{CO})_{2}$

Complex $R \quad E^{\circ}\left(\mathrm{Fc}_{\mathrm{Fc}}{ }^{+}\right)$Ref.

$\begin{array}{llll}\mathrm{Fe}-7 \mathrm{c} & \mathrm{NMeH} & -0.49 \mathrm{~V} & {[310]} \\ \mathrm{Fe}-7 \mathrm{~d} & \mathrm{NMe}_{2} & -0.51 \mathrm{~V} & {[310]} \\ \mathrm{Fe}-7 \mathrm{e} & \mathrm{BPin} & -0.52 \mathrm{~V} & {[310]}\end{array}$

$\begin{array}{llll}\mathrm{Fe}-7 \mathrm{f} & \mathrm{OMe} & -0.52 \mathrm{~V} & {[310]}\end{array}$

Figure 12. Influence of the second coordination sphere on the redox potential. ${ }^{310-312}$

Exchange of the $N$-aryl vs. a more electron rich, aliphatic cyclohexyl substituent moves the reduction potential cathodically by about $100-200 \mathrm{mV}$ (Figure 11). ${ }^{309}$ Variation of the $\mathrm{N}$-aryl ortho-substituents has a much weaker impact on reduction potentials, but can strongly alter rates and equilibria of transition metal complex reactivity. ${ }^{152,295,309,313}$ For example, the common iron $\mathrm{N}_{2}$ precatalyst platform forms $\mathrm{N}_{2}$ bridged dinuclear species with sterically less demanding ortho-aryl substituents at the $N$-phenyl rings. ${ }^{313}$ Amino or boryl substitution in ortho-position, had a rather insignificant effect $(<40 \mathrm{mV})$ on the reduction potential of transition metal complexes (Figure 12). ${ }^{310,311}$ Redox fine-tuning was reported by introducing crown ether fragments as $\mathrm{N}_{\text {imine- }}$ substituents. ${ }^{32,314}$ Ion coordination, to the crown ether moiety resulted in an anodic shift of about $50 \mathrm{mV}$ (Figure 12). 312,314

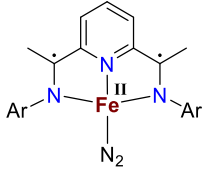

$\mathrm{Fe}-7 \mathrm{a}$

( $\left.{ }^{\mathrm{Pr} P D I}\right) \mathrm{Fe}\left(\mathrm{N}_{2}\right)$

is $\mathrm{L}^{2 \cdot-} \mathrm{Fe} \mathrm{e}^{\mathrm{II}}\left(\mathrm{N}_{2}\right)$

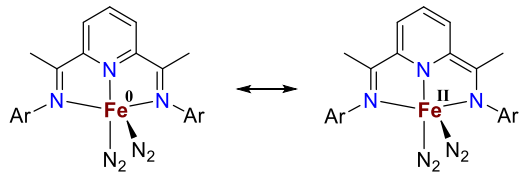

$\mathrm{Fe}-7 \mathrm{j}$

( $\mathrm{Pr} P \mathrm{PDI}) \mathrm{Fe}\left(\mathrm{N}_{2}\right)_{2}$

Is $\mathrm{L}^{0} \mathrm{Fe}^{0}\left(\mathrm{~N}_{2}\right)_{2}$ or

Is $\mathrm{L}^{2-} \mathrm{Fe}^{\mathrm{II}}\left(\mathrm{N}_{2}\right)_{2}$
Figure 13. Electronic structure of the four- and five-coordinate iron PDI dinitrogen complexes. ${ }^{137,153,298}$

The distinct redox activity of the PDI ligand platform necessitates the use of spectroscopic and computational methods for unambiguous oxidation state assignments. For example, Chirik's five-coordinate bisdinitrogen complex (PDI)Fe( $\left.\mathrm{N}_{2}\right)_{2}$ (Fe-7j) exhibits a closed-shell electronic structure, as expressed within ferrous $\left(\left[(\mathbf{P D I})^{2-} \mathrm{Fe}^{\mathrm{II}}\left(\mathrm{N}_{2}\right)_{2}\right]\right)$ or zerovalent iron $\left(\left[(\mathbf{P D I})^{0} \mathrm{Fe}^{0}\left(\mathrm{~N}_{2}\right)_{2}\right]\right)$ resonance structures (Figure 13). ${ }^{137}$ In contrast, the four-coordinate mono- $\mathrm{N}_{2}$ complex (Fe-7a) (best described as Fe(II) with intermediate spin configuration antiferromagnetically coupled to a triplet PDI diradical ligand. ${ }^{137}$ Replacement of the labile dinitrogen ligands by stronger field ligands gives rise to both four and five-coordinate iron( 0 ) complexes. ${ }^{178,315}$ In comparison with the ketimide platform, the iron aldimide system was found to be less electron rich and did not form $\mathrm{N}_{2}$ complexes. ${ }^{316}$ 


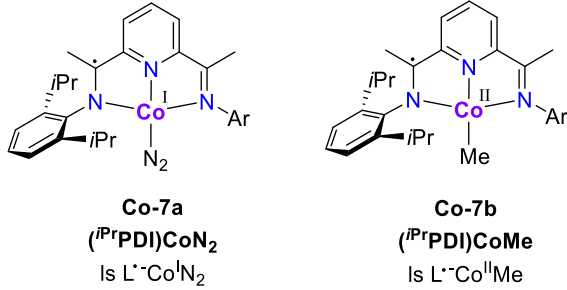

Figure 14. Electronic structure of the cobalt complexes (PDI)CoN $\mathrm{CoN}_{2}$ (Co-7a) and (PDI)CoMe (Co-7b). ${ }^{154,317}$

Reduction of the cobalt(II) high-spin complex (PDI) $\mathrm{CoCl}_{2}$ is ligand centered giving the square-planar $d^{7}$ low-spin complex $\left(\mathbf{P D I}^{-}\right) \mathrm{Co}^{\mathrm{II}} \mathrm{Cl}^{317}$ Antiferromagnetic coupling result in an overall $S=0$ ground states for the chloro and respective alkyl complexes. ${ }^{154,317}$ In analogy, the dinitrogen complex $\left(\right.$ PDI $\left.^{-}\right) \mathrm{Co}^{\mathrm{I}} \mathrm{N}_{2}(\mathbf{C o}-7 \mathbf{a})$ that results from reduction of the chloride under dinitrogen was described as low-spin cobalt(I) with a monoanionic PDI radical ligand. (PDI)CoN $\mathrm{C}_{2}$ $(\mathbf{C o}-7 \mathbf{a})$ is prone to both ligand centered oxidation and reduction, emphasizing the intriguing redox behavior of the PDI ligand. ${ }^{154,167,258,317,318}$ Direct comparison with the analogous iron(II) complex showcases the higher ionization potential of cobalt, which results in a lower oxidation state and less reduced PDI ligand.

In comparison, manganese PDI complexes are less well explored ${ }^{319}($ PDI $) \mathrm{MnX}_{2}(\mathrm{X}=$ halide $)$ and $(\mathbf{P D I}) \mathrm{Mn}(\mathrm{thf})_{2}$ both feature high-spin manganese(II), i.e. in the latter case antiferromagnetically coupled to a dianionic $\mathrm{PDI}^{2 \cdot-}$ diradical ligand, giving an overall $S=3 / 2$ ground state. ${ }^{159}$ These compounds proved inactive as precatalysts in ethylene polymerization, ${ }^{320}$ and most reactions that are catalyzed by iron and cobalt PDI complexes, such as olefin hydrogenation or diene cycloisomerization. In contrast to iron and cobalt, low-valent (PDI)M(N $\left.\mathrm{N}_{2}\right)_{\mathrm{n}}$-type reduction precatalysts with labile dinitrogen ligands could not be obtained, presumably due to weak backbonding from high-spin $\mathrm{Mn}(\mathrm{II})$ to $\mathrm{N}_{2}$. These findings emphasize the accessibility of non-high-spin, low-valent metal states as a prerequisite for redox transformations, such as oxidative addition.
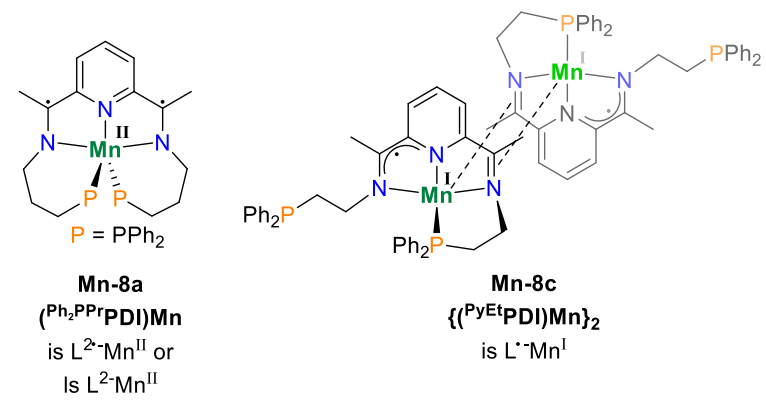

Figure 15. Electronic structures of the five coordinate manganese

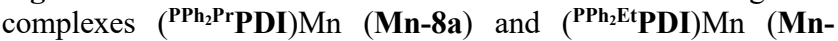
8c). ${ }^{34,301,321}$

Trovitch and coworkers reported important contributions for manganese catalyzed hydroelementation by introduction of modified PDI ligands with two additional diphenylphosphine groups tethered to the imine nitrogen atom (Figure 15). ${ }^{34}$ Two alternative descriptions for the electronic structure of $\left({ }^{\mathbf{P P h}} \mathbf{P r}_{\mathbf{P r}} \mathbf{P D I}\right) \mathrm{Mn}(\mathbf{M n - 8 a})$ were given. Based on
EPR studies the overall doublet ground state was initially attributed to low-spin manganese(II) bound to a dianionic, closed-shell PDI ${ }^{2-}$ ligand. ${ }^{301}$ Subsequent DFT studies gave a possible alternative description as intermediate-spin manganese(II) strongly antiferromagnetically coupled $(J=-$ $401 \mathrm{~cm}^{-1}$ ) to a triplet $\mathrm{PDI}^{2 \cdot-}$ diradical ligand. ${ }^{136}$ Shortening of the propylene linker leads to the dinuclear complex $\left\{\left({ }^{\mathbf{P P h}_{2} \mathrm{Et}} \mathrm{PDI}\right) \mathrm{Mn}\right\}_{2}$ (Mn-8c) (Figure 15) with bridging imine groups as the ethylene bridges prevent coordination of both phosphine side chains. ${ }^{175}$ The overall triplet ground state was rationalized with two intermediate-spin manganese(I) ions that are antiferromagnetically coupled to singly reduced $\mathrm{PDI}^{-}$radical anions. In solution, dissociation of the dimer is proposed.

The general preference for non-high-spin states with this modified PDI platform can be attributed to the additional strong field phosphine donors and gives rise to application in catalysis. ${ }^{34,321,322}$ ( $\left.{ }^{\mathbf{P P h}_{2} \mathbf{P r}} \mathbf{P D I}\right) \mathrm{Mn}(\mathbf{M n - 8 a})$ catalyzes the hydrosilylation of ketones. The hydride complex $\left({ }^{\mathbf{P P h}} \mathbf{P r}^{\mathbf{P}} \mathbf{P D I}\right) \mathrm{MnH}(\mathbf{M n - 8 d})$ was isolated and its role in catalysis investigated. The geometric data suggest a doubly reduced closed-shell PDI ${ }^{2-}$ ligand and low-spin $\mathrm{Mn}^{\mathrm{III}}$, as a consequence of the strong field hydride ligand. In fact, stabilization of the $\mathrm{PDI}^{2-}$ singlet state was also attributed to the strong hydride trans-influence, leading to destabilization of one PDI $\pi^{*}$ acceptor orbital, which favors spin-pairing. ${ }^{136}$
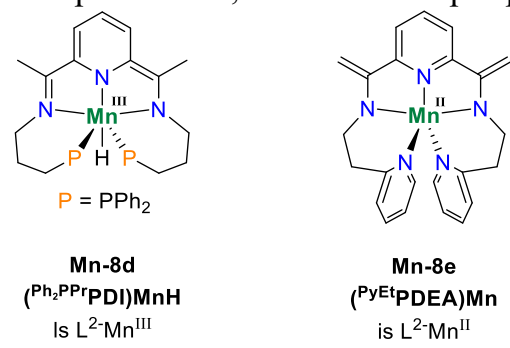

Figure 16. Electronic structures of manganese complexes ( ${ }^{\text {PPh }}{ }_{2}$ Pr PDI) MnH and ( ${ }^{\text {PyEt PDEA }) M n . ~}{ }^{34,136,323}$

The phosphine side chains were also replaced by ethylene pyridine arms. ${ }^{323}$ Reduction of a dihalide precursor with excess $\mathrm{Na} / \mathrm{Hg}$ gave a five-coordinate bisenamide species (Figure 16). Deprotonation of the methyl groups truncates the PDI $\pi$-system thereby raising the $\pi^{*}$ acceptor levels. This ligand is therefore not redox-active. This complex is best described as intermediate-spin $\mathrm{Mn}^{\mathrm{II}}$ with a closed-shell bisenamido PDI ligand. Notably, other deprotonated PDI manganese complexes were shown to undergo dimerization by $\mathrm{C}$ $\mathrm{C}$ bond formation. ${ }^{324}$

Nickel PDI complexes are comparatively scarcely examined. Wieghardt and co-workers examined a series of octahedral complexes $(\mathbf{P D I})_{2} \mathrm{M}\left(\mathrm{PF}_{6}\right)_{\mathrm{n}}(\mathrm{M}=\mathrm{Mn}, \mathrm{Fe}, \mathrm{Co}, \mathrm{Ni}, \mathrm{Cu}$, $\mathrm{Zn}){ }^{325}$ A four-coordinate nickel PDI complex was first reported by Manuel and Rohde. ${ }^{326}$ The distorted square-planar complex (PDI)NiCl (Ni-7a) was described as nickel(II) and a $\mathrm{PDI}^{-}$radical ligand. Reduction with $\mathrm{NaH}$ under $\mathrm{N}_{2}$ leads to the square-planar complex $(\mathbf{P D I})^{2-} \mathrm{Ni}^{\mathrm{II}}\left(\mathrm{N}_{2}\right)(\mathbf{N i}-7 \mathbf{b})$ with a closed shell dianionic ligand. ${ }^{167}$ 
In contrast, the bisaldimine PDI complexes (PDI)NiX (X = $\mathrm{Cl}, \mathrm{Br}$ ) are best described as nickel(I) species. ${ }^{327}$ The $\mathrm{d}^{9}$ configuration is associated with distortion from square-planar geometry. Unlike Rohde's system this complex does not undergo ligand centered oxygenation reaction by air as a consequence of the metal centered radical character.
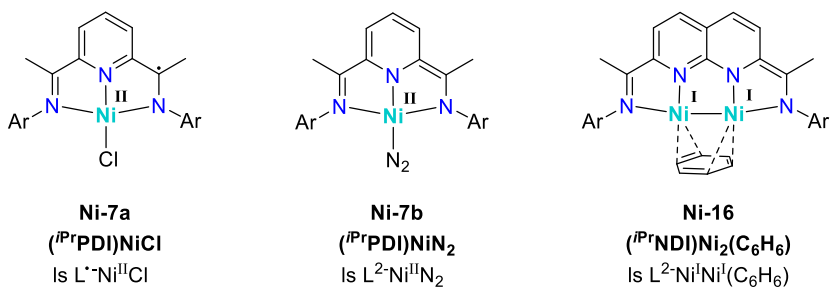

Figure 17. Electronic structures of complex different nickel PDI and NDI complexes. ${ }^{167,326,328}$

Uyeda and coworkers expanded this ligand platform to the to naphtyridinediimine (NDI; Figure 17), which offers two adjacent bonding sites for the stabilization of bimetallic complexes. Furthermore, the extended $\pi$-system enables improved electron storage. (NDI) $\mathrm{Ni}_{2}\left(\mathrm{C}_{6} \mathrm{H}_{6}\right)$ (Ni-16) (Figure 17) was described as a dinickel(I) complex with the two metal ions in a bonding distance $\left(D_{\mathrm{Ni}-\mathrm{Ni}}=2.496(1) \AA\right)$ bound to a dianionic $\mathrm{NDI}^{2-}$ ligand. Remarkably, this compound exhibits four ( 2 metal- and 2 ligand-centered) reversible reductions between 0 and $-3.0 \mathrm{~V}$ (vs. $\mathrm{Fc} / \mathrm{Fc}^{+}$), pointing out the ability of this platform to undergo multi-electron redox processes within accessible potentials. ${ }^{328}$

The analogous iron and cobalt complexes, $\left[(\mathbf{N D I}) \mathrm{M}_{2}\left(\mathrm{C}_{6} \mathrm{H}_{6}\right)\right]$ $(\mathrm{M}=\mathrm{Fe}, \mathrm{Co})$, were subsequently reported. ${ }^{329}$ In contrast to the nickel and the cobalt complexes ( $S=0$ ground state) the iron complex exhibits four unpaired electrons $(S=2$ ground state).

2.2.3. Terpyridine The scope of applications for the terpy ligand is more diverse than for PDI and Pybox ligands, including many photochemical applications, ${ }^{43,330-336}$ but also as building block in supramolecular chemistry, ${ }^{337}$ ${ }^{340}$ magnetochemistry, ${ }^{341}$ and catalysis. ${ }^{1,4,73}$ Moreover, the role of terpy redox activity in nickel catalyzed $\mathrm{C}-\mathrm{C}$ bond formation was recently discussed. ${ }^{342-346}$

PDI and terpy ligands show similar redox properties. Although the terpy ligand class (tpy $=2,2^{\prime}: 6^{\prime}, 2^{\prime}$ '-terpyidine) is widely established and utilized for decades, redox non-innocent ligand behavior was evaluated more recently. ${ }^{347}$ The free terpy ligand exhibits a reversible reduction at $-2.55 \mathrm{~V}$ (vs. $\mathrm{Fc} / \mathrm{Fc}^{+}$) and an irreversible reduction event at around $3.06 \mathrm{~V}$ (Figure 18). ${ }^{348,349}$ As an example of ligand non-innocence, the four reduction events of the chromium complex $\left[\mathrm{Cr}^{\mathrm{III}}(\mathbf{t p y})_{2}\right]$ are all ligand centered as shown by K-edge Xray absorption spectroscopy and DFT calculations. ${ }^{350} \mathrm{In}$ contrast, the homoleptic cobalt complexes $\left[\mathrm{Co}(\mathbf{t p y})_{2}\right]^{\mathrm{n}+}$ prefer metal based redox steps. ${ }^{351}$ Palladium and platinum complexes of the related 2,5-bis( $\alpha$-pyridyl)pyrrolate ligand also showed ligand based redox events which are cathodically shifted due to the anionic ligand character. ${ }^{352}$ Redox non-innocence was reported to stabilize unusual oxidation states, like an square pyramidal Ni(III) species. ${ }^{353}$ Analogous triazol-based ligands were also reported..$^{354,355}$

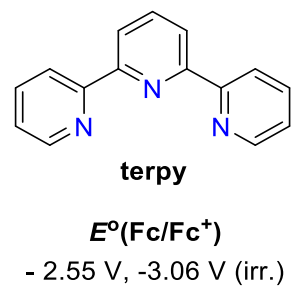

Figure 18. terpy ligand and its redox potential. ${ }^{349}$

The close energetic proximity of the terpy acceptor orbitals with respect to nickel d-levels is showcased by EPR spectroscopic examination of the complexes (tpy)NiX $(X=M e$, $\mathrm{Br}$ ). The strong-field methyl ligand results in terpy reduction, $\left(\mathbf{t p y}^{-}\right) \mathrm{Ni}^{\mathrm{II}} \mathrm{Me}(\mathbf{N i}-\mathbf{9})$, while (tpy) $\mathrm{Ni}^{\mathrm{I}} \mathrm{Br}(\mathbf{N i - 1 0})$ is best described as a nickel(I) complex with neutral terpy. ${ }^{356,357}$ Both nickel complexes show similar electrochemical behavior.
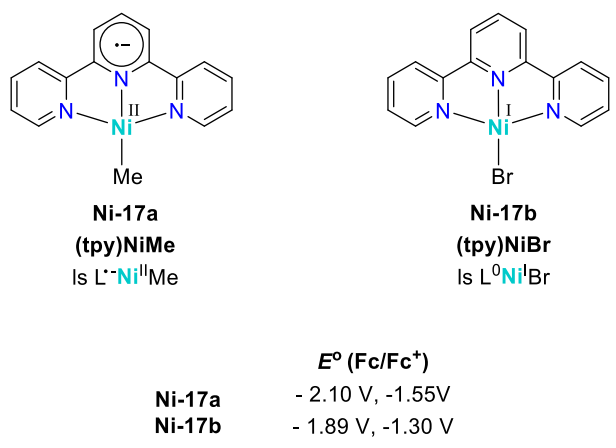

Figure 19. Electronic structure of the nickel terpy complexes (tpy)NiMe (Ni-17a) ${ }^{356}$ and (tpy)NiBr (Ni-17b) ${ }^{358}$ and the corresponding redox potentials (potentials were re-referenced to the $\mathrm{Fc} / \mathrm{Fc}^{+}$couple according to ref 359$)$.

Chirik and coworkers reported the divalent iron complex (tpy) $\mathrm{Fe}\left(\mathrm{CH}_{2} \mathrm{SiMe}_{3}\right)_{2}(\mathbf{F e}-\mathbf{1 7})$ as a precatalyst for olefin hydrosilylation (Figure 20). ${ }^{360}$ Magnetochemical, spectroscopic and computational evaluation support the description as a high-spin ferric complex with an antiferromagnetically coupled, anionic terpy radical ligand resulting in an overall $S=2$ ground-state. On the contrary, the related complex (tpy) $\mathrm{Co}\left(\mathrm{CH}_{2} \mathrm{SiMe}_{3}\right)(\mathbf{C o}-\mathbf{1 7})$, which is a hydroboration catalyst, exhibits an $S=0$ ground-state as a result of low-spin cobalt(II) coupled to the terpy=- radical ligand (Figure 20). ${ }^{361}$

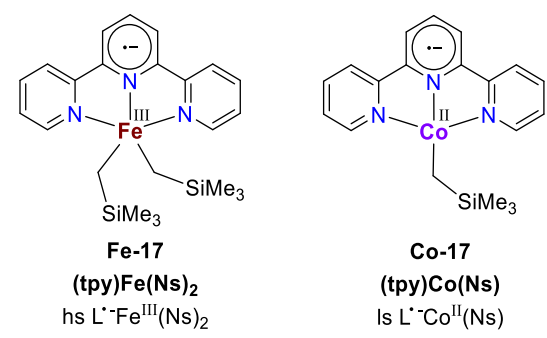

Figure 20. Terpy complexes of iron and cobalt and their electronic structure. ${ }^{360,361}$ 
2.2.4 Pybox Bis(pyridyl)oxazoline (Pybox) complexes were extensively utilized in asymmetric catalysis, as this ligand allows for the synthetic introduction of chiral moieties at the oxazoline framework with strong stereochemical induction. ${ }^{362-367}$ In contrast, the electronic structures of Pybox bearing (pre)catalysts are less well explored. Comparison of Pybox and PDI as free ligands and within coordination compounds was initially carried out by Chirik and coworkers. ${ }^{309}$ Reduction potentials of several free PDI and Pybox ligands were found within $20 \mathrm{mV}$. However, comparison of the $\mathrm{CO}$ stretching vibrations of the complexes $\left[(\mathbf{L}) \mathrm{Fe}(\mathrm{CO})_{2}\right](\mathbf{L}=$ iPrPybox, PDI) showed lower values for the Pybox complexes by about $40 \mathrm{~cm}^{-1}$, pointing out stronger donation by this pincer ligand. Importantly, $(\mathbf{P y b o x}) \mathrm{FeCl}_{2}$ does not react with dinitrogen under reductive conditions but forms the bischelate species $(\mathbf{P y b o x})_{2} \mathrm{Fe}$. This reaction was pointed out as a catalyst deactivation pathway in ketone hydrosilylation. ${ }^{309}$

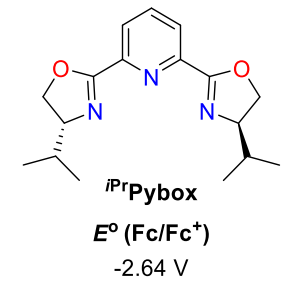

Figure 21. Pybox and the corresponding redox behavior. ${ }^{309}$

The similarities between PDI, terpy, and Pybox ligands was further illustrated by ${ }^{57} \mathrm{Fe}$ Mößbauer spectroscopic examination of a series of five-coordinate dialkyl iron complexes. ${ }^{309,360} \quad\left({ }^{\text {Pr }} \mathrm{Pybox}\right) \mathrm{Fe}\left(\mathrm{CH}_{2} \mathrm{SiMe}_{3}\right)_{2}$, (terpy) $\mathrm{Fe}\left(\mathrm{CH}_{2} \mathrm{SiMe}\right)_{2}$, and ( $\left.{ }^{\mathrm{Et}} \mathrm{PDI}\right) \mathrm{Fe}\left(\mathrm{CH}_{2} \mathrm{SiMe}_{3}\right)_{2}$ all feature high-spin iron(III), antiferromagnetically coupled to pincer ligand radical anions. Schley and Fu conducted a mechanistic study on $\mathrm{Ni}(\mathbf{P y b o x})$-catalyzed, stereoselective Negishi cross-coupling, and defined (Pybox) $\mathrm{NiPh}$ as $\mathrm{Ni}^{\mathrm{II}}$ with a pincer radical anion. ${ }^{368}$ In general, the relevance of redox non-innocence for catalysis is less well explored for Pybox.

2.2.5. Pyridyldicarbenes Most reported CNC pincer complexes feature $4 \mathrm{~d}$ and $5 \mathrm{~d}$ metals. ${ }^{369}$ Cobalt and iron complexes with the strongly donating pyridyldicarbene pincer ligands first reported by Danopoulos, ${ }^{370-372}$ and evaluated in catalysis in a comparative study by Chirik and co-workers. ${ }^{289,373,374}$
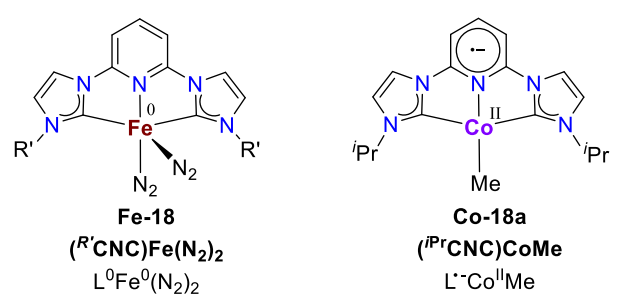

Figure 22. Iron and Cobalt pyridyldicarbene (CNC) pincer complexes. ${ }^{289,373,374}$

The $\mathrm{N}_{2}$-stretching vibrations of five-coordinate iron dinitrogen complexes are shifted to smaller wavenumbers by about
$20 \mathrm{~cm}^{-1}$ for the CNC series. ${ }^{298,370}$ The free CNC-pincer showed no accessible electrochemical reduction but could be reduced chemically, as determined by EPR-spectroscopy. ${ }^{374}$ Unlike for (PDI)Fe $\left(\mathrm{N}_{2}\right)_{2}$, dissociation of a dinitrogen ligand was not found in solution. Detailed spectroscopic and computational analysis are in agreement with iron( 0 ) and a neutral dicarbene pincer ligand. ${ }^{289}$ The cobalt methyl complex (Figure 22) is a low spin $\mathrm{Co}^{\mathrm{II}}$ species with an antiferromagnetically coupled radical ligand. ${ }^{374}$ The radical ligand undergoes side reactions like alkyl migration onto the ligand backbone. $^{375}$

\subsubsection{Iminopyridine and Related Systems}

Bidentate iminopyridine (IP) ligands, which exhibit a truncated $\pi$-system with respect to PDI, can also be redox noninnocent. This is illustrated by a series of tetrahedral $3 \mathrm{~d}$ metal complex (IP) ${ }_{2} \mathrm{M} \cdot{ }^{376}$ Huang and coworkers developed an iminopyridine-based pincer ligand extended by one additional phosphinite arm (Figure 23). ${ }^{377}$ The free ligand can be reversibly reduced at $-2.74 \mathrm{~V}\left(\mathrm{vs}\right.$. $\mathrm{Fc} / \mathrm{Fc}^{+}$), i.e. a mild cathodic shift of $120 \mathrm{mV}$ with respect to PDI, assigning to the phosphine moiety, which is a general stronger donating group in contrast to imine group in the PDI ligand. In a series of iron carbonyl complexes the $\mathrm{C}-\mathrm{O}$ stretching vibration of a PNN complex was bathochromically shifted by approximately $100 \mathrm{~cm}^{-1}$ with respect to the corresponding PDI complex. ${ }^{378}$
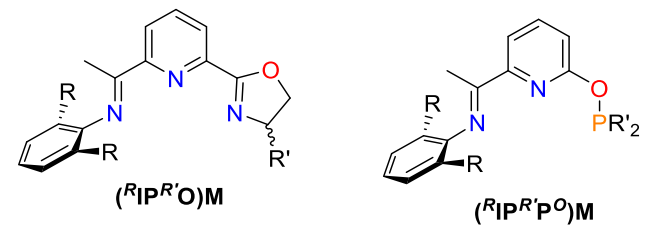

$$
\begin{array}{lc} 
& \boldsymbol{E}^{\circ}\left(\mathbf{F c} / \mathrm{Fc}^{+}\right) \\
\mathrm{I}^{\mathrm{PPr}} \mathrm{P}^{t \mathrm{Bu}} \mathrm{P}^{\mathrm{O}} & -2.74 \mathrm{~V} \\
\mathrm{R}_{\mathrm{IP}} \mathrm{R}^{\prime} \mathrm{O} & \text { not investigated }
\end{array}
$$

Figure 23. Oxazoline iminopyridine and phosphinite iminopyridine and their redox potential. ${ }^{377}$

Complexation with ferrous chloride gives the five coordinate $d^{6}$ high-spin species $\left({ }^{t \mathbf{B u}} \mathbf{P N N}{ }^{i \mathbf{P r}}\right) \mathrm{FeCl}_{2}$. The reduced dicarbonyl complex $\left.{ }^{\left({ }^{(B u} \mathbf{P N N}\right.}{ }^{i P r}\right) \mathrm{Fe}(\mathrm{CO})_{2}$ exhibits a reversible reduction at $-2.54 \mathrm{~V}$, i.e. slightly lower than the PDI congener. ${ }^{308}$ In analogy to the PDI system, DFT computations support iron(I) with a singly reduced pincer ligand. ${ }^{377}$ However, low-valent dinitrogen complexes were not reported and catalytic experiments were only performed by in situ reduction. Due to the potential lability of the phosphinite group with respect to P-O cleavage the oxygen linker was also replaced by a methylene group. ${ }^{379}$ Alternatively, Huang and coworkers reported the synthesis of a bipy-based PNN-pincer with a phosphine side arm. The redox properties of these ligands have not been investigated in detail..$^{380}$

The same group developed an NNN-pincer ligand that combines the PDI and the chiral Pybox frameworks (Figure 23). ${ }^{381}$ This ligand has been applied to manganese-, iron- and cobalt-catalyzed asymmetric hydrogenation, hydroboration and hydrosilylation. ${ }^{382-386}$ Electronic structure investigations of catalytically relevant model complexes were not reported. 
2.2.7. Bipyridine Based Pincer Ligands Redoxactive 2,2'-bipyridine ${ }^{347,387-389}$ ligand has been introduced as a building block for PNN and NNN pincer ligands. The phosphine tethered bipyridine ligand ${ }^{\mathbf{P h}} \mathbf{B P P}$ (Figure 24) was utilized in copper catalyzed $\mathrm{CO}_{2}$ electroreduction. ${ }^{390}$ Facile dearomatization of an iron complex was reported by Milstein and co-workers. ${ }^{391}$ The electronic properties of ${ }^{\mathbf{R}, \mathbf{R}} \mathbf{B P I}$ with respect of ligand centered redox-activity are yet to be explored. ${ }^{392}$
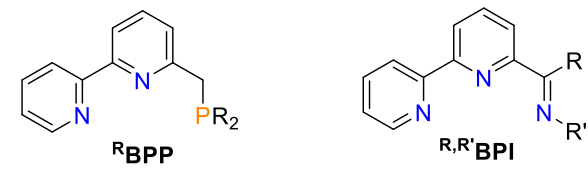

Figure 24. Bipyridine based pincer complexes. ${ }^{390,392}$

\subsubsection{Azaphenyl Ligands and Related Systems} The scope of tridentate NNN-type ligands was extended by Goswami upon attaching phenylazo groups to a central pyridine core (Figure 25) ${ }^{393-395}$ In analogy to the PDI platform, a low lying, energetically accessible $\pi^{*}$ orbital is located at the azo unit. The free PDAz ligand exhibits two reversible reductions at $-1.79 \mathrm{~V}$ and $-1.47 \mathrm{~V}$ (vs. $\mathrm{Fc} / \mathrm{Fc}^{+}$), respectively,.i.e. is a large anodic shift of around $750 \mathrm{mV}$ with respect to the PDI platform. The one-electron-reduced radical ligand could be isolated from bulk electrolysis. ${ }^{396}$

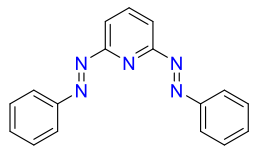

PDAz

$E^{\circ}\left(\mathbf{F c} / \mathbf{F c}^{+}\right)$
$-1.79 \vee,-1.47 \mathrm{~V}$

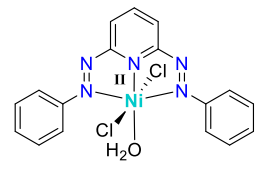

$\mathrm{Ni}-19 \mathrm{a}$ (PDAz) $\mathrm{NiCl}_{2}\left(\mathrm{H}_{2} \mathrm{O}\right)$ Is $\mathrm{L}^{0} \mathrm{Ni}^{\mathrm{II}[\mathrm{x}]}$

$E^{\circ}\left(\mathrm{Fc} / \mathrm{Fc}^{+}\right)$
$-1.69 \vee$

$-1.69 \mathrm{~V},-1.43 \mathrm{~V}$
$-0.57 \mathrm{~V},-0.44 \mathrm{~V}$

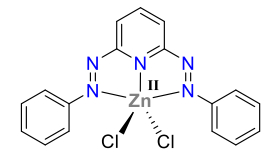

$\mathrm{Zn}-19$ (PDAz) $\mathrm{ZnCl}_{2}$ Is $L^{0} \mathrm{Zn} n^{\mathrm{II}}[\mathrm{x}]$ $E^{\circ}\left(\mathrm{Fc} / \mathrm{Fc}^{+}\right)$ $-1.17 \mathrm{~V},-0.59 \mathrm{~V}$
Figure 25. Electronic properties and redox potentials of $\mathbf{P D A z}{ }^{396}$ and corresponding nickel(II) ${ }^{175}$ and zinc(III) ${ }^{174}$ complexes.

An octahedral, high-spin nickel(II) complex with this ligand (Ni-19a, Figure 25) was used as an alcohol dehydrogenation catalyst, which is discussed in Section 4.1.2. ${ }^{175}$ Four reversible and quasi-reversible reductions were found in a range between $-0.44 \mathrm{~V}$ and $-1.69 \mathrm{~V}$ and assigned to stepwise population of the ligand $\pi^{*}$ orbitals. Two reversible reductions at -1.17 and $-0.59 \mathrm{~V}$ were obtained for the corresponding zinc complex (Zn-19). ${ }^{174}$

The combination of phenylazo and phenanthrolin building blocks gave rise to the PhenAz ligand (Figure 26). This NNN-pincer ligand exhibits a reversible reduction at $1.39 \mathrm{~V}$ suggesting potential redox non-innocence. Introduction of a para-chloro substituent at the phenyl moiety only slightly shifts the reduction potential $\left(\Delta E^{\mathrm{o}}=0.02 \mathrm{~V}\right)$. Unlike the symmetric PDAz ligand, this ligand shows one reversible and one irreversible reduction. ${ }^{397}$

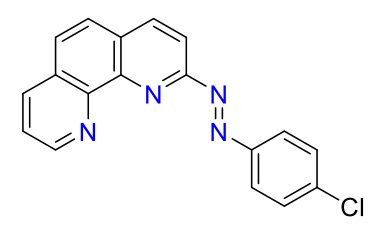

PhenAz

$E^{\circ}\left(\mathrm{Fc} / \mathrm{Fc}^{+}\right)$

$-1.83 \vee$ (irr.), $-1.39 \mathrm{~V}$

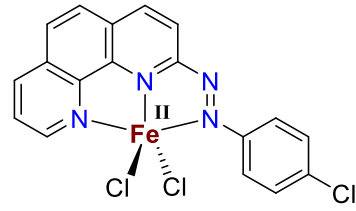

Fe-20

(PhenAz) $\mathrm{FeCl}_{2}$ hs $\mathrm{L}^{0} \mathrm{Fe} \mathrm{II}^{\mathrm{II}]}$

$E^{\circ}\left(\mathrm{Fc} / \mathrm{Fc}^{+}\right)$

$-1.23 \mathrm{~V}$ (quasi irr.), $-0.71 \mathrm{~V}$
Figure 26. Redox potentials of ligand $\mathbf{P h e n} \mathbf{A} \mathbf{z}^{397}$ and a ferric iron complex (potentials were re-referenced to the $\mathrm{Fc} / \mathrm{Fc}^{+}$couple according to ref 359$).{ }^{397}$

Complexation with ferrous chloride leads to a five-coordinate dichloride that was described as iron(II) high-spin coordinated to a neutral pincer ligand. This compound exhibits a reversible reduction at $-0.71 \mathrm{~V}$ and irreversible reduction at $-1.23 \mathrm{~V}$ (vs. $\mathrm{Fc} / \mathrm{Fc}^{+}$). Both the free ligand and the iron complex $(\mathbf{F e}-20)$ undergo reduction in the presence of $\mathrm{KO} t \mathrm{Bu}$. In both cases, isotropic EPR spectra with similar g-values ( $g$ $=1.997,2.004$ ) were obtained suggesting ligand centered reduction in the latter case. The possible relevance for catalytic dehydrogenation of secondary alcohols is discussed below. ${ }^{397}$

2.2.9. Bis(pyrazolyl)pyridine The Bis(pyrazolyl)pyridine (PDPz) was first characterized electrochemically by Caulton and co-workers. ${ }^{398}$ Within a potential window of more than $4 \mathrm{~V}$ no reversible redox events were observed reflecting the electron rich character in comparison to the PDI ligand. This is confirmed by the $\mathrm{CO}$ stretching vibration of corresponding zerovalent iron complexes. Accordingly, redox activity of the pincer was not observed. However, the deprotonated ligand (for $\mathrm{R}^{\prime}=\mathrm{H}$ ) can serve as a proton relay in metal-ligand cooperative $\mathrm{H}-\mathrm{X}$ heterolysis ${ }^{399}$ and was $\mathrm{N}$ borylated by HBpin in case of a related PNN pincer. ${ }^{400}$ The related bis(pyrrolide) pincer ligand exhibits no reductive features and three irreversible oxidative redox events in the CV. ${ }^{401}$

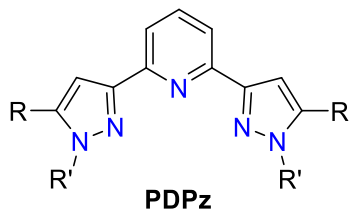

Figure 27. Bis(pyrazol)pyridine as pincer ligand. ${ }^{402}$

\subsubsection{Phosphaalkene Pincer Ligands}

The phosphaalkene pincer ligand BPEP is less explored and has significantly different properties than the isoelectronic PDI due to very low lying $\pi^{*}$-orbital. ${ }^{403}$ Electrochemical characterization of a benzodiphosphoalkene pincer revealed reversible reduction. ${ }^{404}$ Ozawa and Nakajima reported the synthesis of the iron complex (BPEP)FeBr, which features high-spin iron(I) with a strongly distorted trigonal monopyramidal structure. ${ }^{405}$ This contrasts with the corresponding 
square-planar iron(II) PDI complex. To date, only a handful reactivity studies have been exemplified, ${ }^{406-408}$ catalytic studies have not been carried out yet.

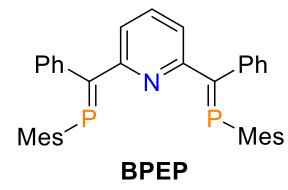

Figure 28. Bis(phosphaethenyl)ligands. ${ }^{405}$

\section{HYDROGENATION}

\subsection{Hydrogenation of Ketones and Aldehydes}

\subsubsection{Pyridyl-Based and Related Pincer Cata-}

lysts The first iron pincer catalyst for ketone hydrogenation was reported in 2011 by Langer et al. (Scheme 15). ${ }^{107}$ The

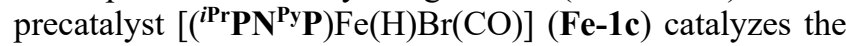
reduction of several ketones in ethanol with low catalyst loading at mild conditions ( $4.1 \mathrm{~atm} \mathrm{H}_{2}$, r.t.) upon activation with base $(\mathrm{KO} t \mathrm{Bu})$. High turnover numbers $(1880 @$ r.t.; $\left.1720 @ 40{ }^{\circ} \mathrm{C}\right)$ and frequencies $\left(430 \mathrm{~h}^{-1} @ 40^{\circ} \mathrm{C}\right)$ were achieved for the benchmark substrate acetophenone. While electronic effects by the arylketone para-substituents (-Me, $-\mathrm{Cl})$ were small, potentially donating groups $\left(-\mathrm{CN},-\mathrm{NH}_{2}\right)$, strongly inhibit catalysis. $\alpha, \beta$-Unsaturated ketones exhibit low chemoselectivity and aliphatic ketones like cyclohexanone give medium yields under these conditions.

Scheme 15. Iron-catalyzed ketone hydrogenation reported by Langer et al. ${ }^{107}$ a: $0.1 \mathrm{~mol} \%$ catalyst and $0.2 \mathrm{~mol} \% \mathrm{KO} t \mathrm{Bu}$.

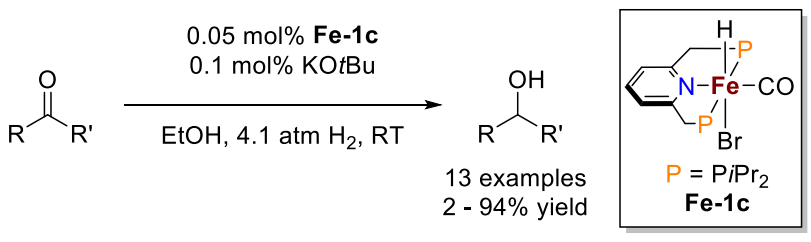

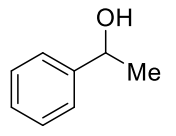

$94 \%$ yield TON $=1880$

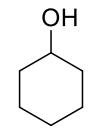

$64 \%$ yield TON $=1280$

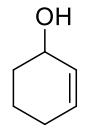

$2 \%$ yield $^{a}$<smiles>CC(O)c1ccc(N)cc1</smiles>

$0 \%$ yield
Langer et al. later expanded this catalyst platform by the borohydride analogue $\left.\left[{ }^{\left({ }^{\mathbf{P r}} \mathbf{P N}\right.}{ }^{\mathbf{P y}} \mathbf{P}\right) \mathrm{Fe}(\mathrm{H})(\mathrm{CO})\left(\eta^{1}-\mathrm{HBH}_{3}\right)\right](\mathbf{F e}-$ 1d) which enables catalysis under base-free conditions. However, low catalytic activity is obtained at room temperature due to the necessity of thermal borane dissociation for catalyst activation. ${ }^{409}$ Similar catalytic activities are obtained for Fe-1c at r.t. and $\mathbf{F e}-1 \mathbf{d}$ at $40{ }^{\circ} \mathrm{C}$, respectively $(0.05 \mathrm{~mol} \%$; $\left.\mathrm{TOF}_{\mathrm{Fe}-1 \mathrm{c}}=430 \mathrm{~h}^{-1}, \mathrm{TOF}_{\mathrm{Fe}-1 \mathrm{~d}}=296 \mathrm{~h}^{-1}\right)$. The substrate scope is similar, yet with remarkable catalytic activity for 2acetylpyridine in case of Fe-1d.

Fe-10a/Fe-11 are also active catalysts for aldehyde hydrogenation (Table 1). ${ }^{107,409}$ Considerably lower turn-over numbers were initially obtained under the same conditions $\left(\mathrm{TON}_{\mathrm{PhC}(\mathrm{O}) \mathrm{H}}=\mathrm{ca} .300\right)$. The authors attributed the reduced yield to catalyst inhibition upon formation of benzoic acid via a base-catalyzed Cannizzaro reaction. This rationale lead to strongly improved catalytic performance by addition of base to prevent catalyst inhibition by acidolysis. ${ }^{410}$ Accordingly, in the presence of excess $\mathrm{NEt}_{3}\left(30 \mathrm{~atm} \mathrm{H}_{2}\right)$ the TON for benzaldehyde hydrogenation could be improved by more than an order of magnitude (Table 1). This protocol gives excellent yields for the hydrogenation of secondary and tertiary aliphatic as well as aromatic aldehydes with low catalyst loadings $(0.025 \mathrm{~mol} \%)$. Primary aliphatic aldehydes are not suitable substrates due to competing aldol condensation. Low yields for 4-bromo- and 4-chlorobenzaldehyde are attributed to oxidative substrate esterification with the solvent to give the respective ethylbenzoates.

Stoichiometric control experiments were conducted to examine the reaction mechanism. ${ }^{107}$ Deprotonation of the pincer backbone arm requires protic solvents and an additional ancillary ligand like benzonitrile or benzophenone. The resulting dearomatized iron hydride complex rapidly adds dihydrogen to a rearomatized trans-dihydride complex. These results led to a proposed all-iron(II) inner-sphere mechanism (Scheme 16) via substrate coordination $(\mathbf{A} \rightarrow \mathbf{B})$, migratory insertion $(\mathbf{B} \rightarrow \mathbf{C})$, metal-ligand cooperative $\mathrm{H}_{2}$-heterolysis $(\mathbf{C} \rightarrow \mathbf{D})$ and product elimination $(\mathbf{D} \rightarrow \mathbf{A})$.

Table 1. Catalytic hydrogenation of benzaldehyde with the iron PNP pincer precatalysts Fe-1c and Fe-1d. ${ }^{107,409,410}$

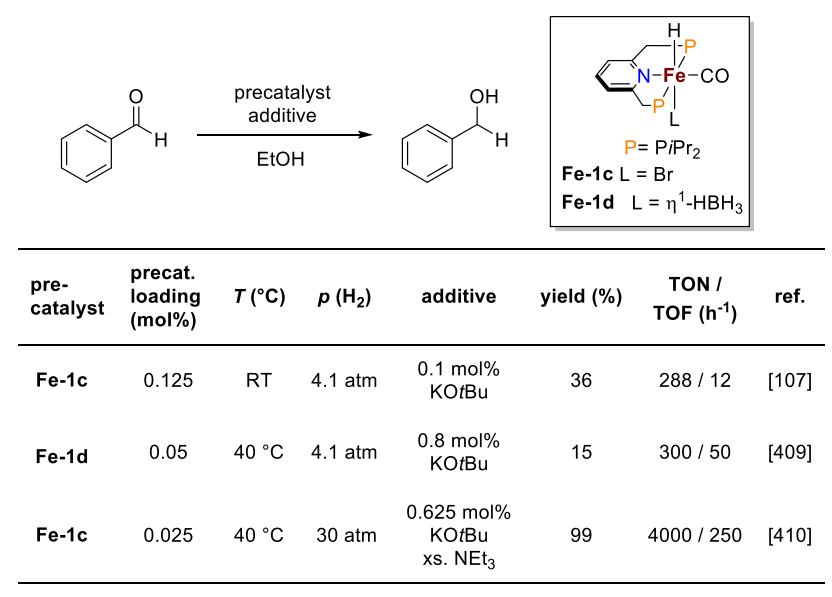

This mechanism was later challenged by Yang within a DFT study on a $\mathrm{PMe}_{2}$-truncated model system. ${ }^{411}$ The author proposes the trans-dihydride complex $\left[\left(\mathbf{P N}^{\mathbf{P y}} \mathbf{P}\right) \mathrm{Fe}(\mathrm{H})_{2} \mathrm{CO}\right]$ (Fe-1e) as the active catalyst species that reduces the substrate by outer-sphere hydride transfer. Importantly, the relevance of metal-ligand cooperation for catalysis was dismissed. Furthermore, EtOH solvent catalyzed $\mathrm{H}_{2}$ heterolysis via an iron(II) ethanolate complex was proposed to accelerate dihydrogen activation. 

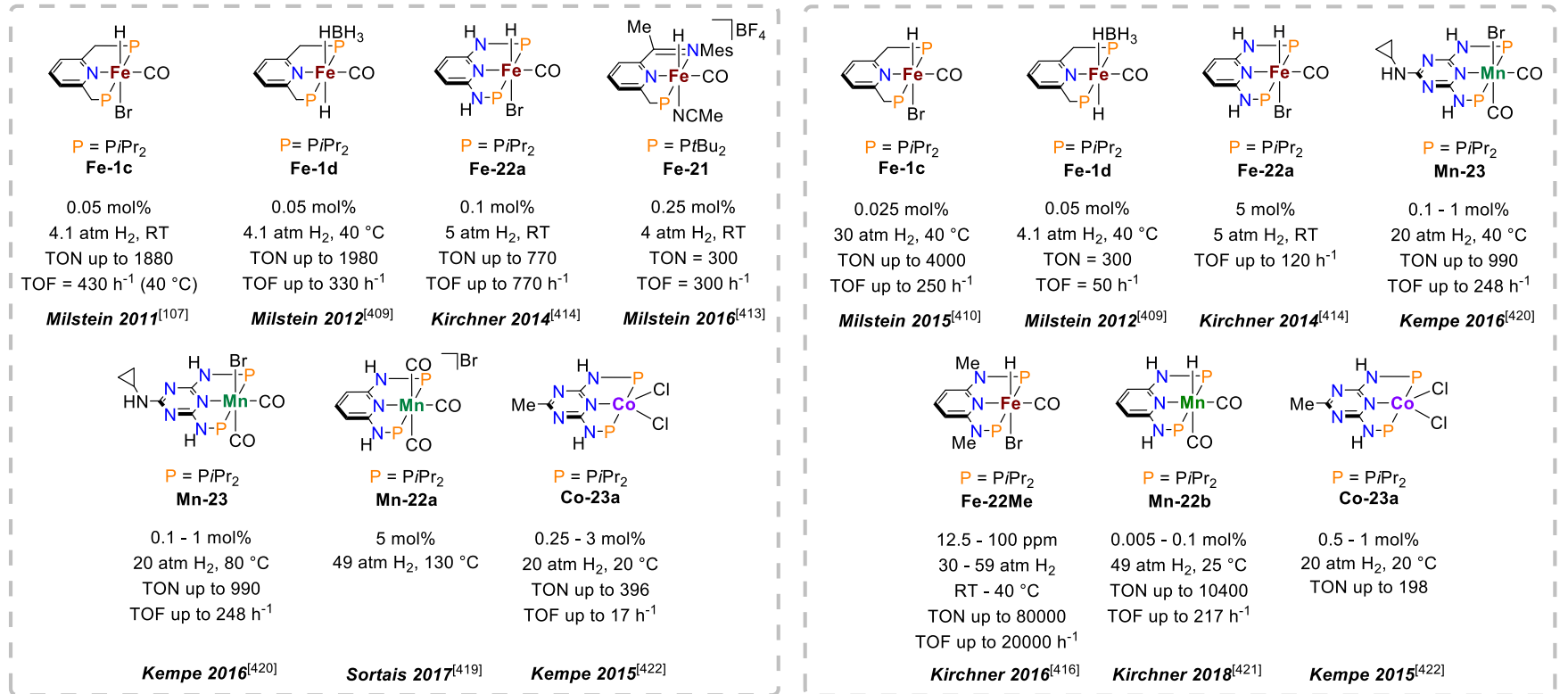

Figure 29. Ketone and aldehyde hydrogenation preatalysts with diphosphinopyridyl and related ligands.

Scheme 16. Proposed MLC-assisted mechanism for the hydrogenation of ketones with iron $\mathbf{P}^{\mathbf{P y}} \mathbf{N P}$ pincer catalysts. ${ }^{107}$

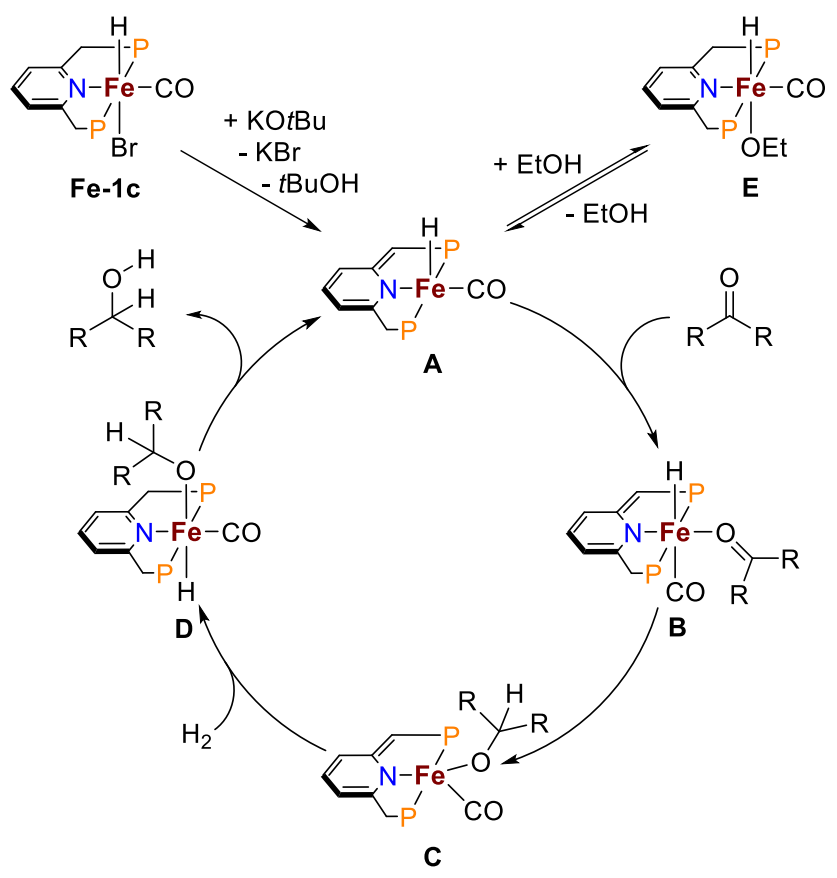

In response, Milstein and co-workers pointed out that the in situ generated trans-dihydride Fe-1e does not react with acetophenone over an extended timescale in the absence of $\mathrm{H}_{2}$ and therefore dismissed its relevance for catalysis. ${ }^{409}$ An alternative iron(II)/iron(0) cycle based on the full DFT model with explicit participation of the solvent $(\mathrm{EtOH})$ as proton shuttle was proposed (Scheme 17). Rearomatization of the iron(II) hydride $\mathbf{A}$ was described as reductive elimination giving the four-coordinate iron $(0)$ species $\mathbf{F}$. After coordination of ethanol $(\mathbf{G})$, concerted 2-proton/2-electron transfer from the pincer backbone and coordinated ethanol to the substrate restores iron(II) as the dearomatized ethanolate complex I. Regeneration of parent A was proposed to proceed by hydrogenolysis of the iron ethanolate moiety via the two possible configurations of the dihydrogen intermediate $(\mathbf{J} / \mathbf{L})$ with low barriers and overall energetic span for the full cycle of $21.7 \mathrm{kcal} \cdot \mathrm{mol}^{-1}$.

Morello and Hopmann recently reevaluated these mechanisms computationally (full model, dispersion correction, PCM solvation model, one explicit EtOH as proton shuttle). ${ }^{412}$ The authors considered the solvent-assisted iron(0)/iron(II) redox cycle (Scheme 18, a) and three alliron(II) mechanisms via the originally proposed active monohydride catalyst (Scheme 18, b), Yang's dihydride pathway without metal-ligand cooperativity (Scheme 18, c) and a dihydride route with pincer assisted $\mathrm{H}_{2}$-heterolysis and proton shuttling (Scheme 18, d). The Fe(II)/Fe(0) mechanism (path a) was dismissed based on the high computed effective barrier for reductive elimination $\left(52.5 \mathrm{kcal} \cdot \mathrm{mol}^{-1}\right.$ or 37.5 $\mathrm{kcal} \cdot \mathrm{mol}^{-1}$ with proton shuttling). Similarly, path $\mathrm{b}$ exhibits high effective barriers for the inner-sphere hydride transfer (38.0 $\mathrm{kcal} \cdot \mathrm{mol}^{-1}$ for acetophenone) and subsequent proton transfer $\left(32.9 \mathrm{kcal} \cdot \mathrm{mol}^{-1}\right)$. Finally, mechanisms c $(20.4$ $\left.\mathrm{kcal} \cdot \mathrm{mol}^{-1}\right)$ and $\mathrm{d}\left(20.1 \mathrm{kcal} \cdot \mathrm{mol}^{-1}\right)$ are close in effective barrier. Notably, the scenario with the most favorable energy profile (d) combines Milstein's original proposal (metal-ligand cooperative $\mathrm{H}_{2}$ activation) with Yang's modification (dihydride catalyst with outer-sphere substrate activation). 
Scheme 17. Proposed iron(II)/iron(0) redox mechanism for the acetophenone hydrogenation $\left(\mathrm{P}=\mathrm{PiPr}_{2}\right) .{ }^{409}$

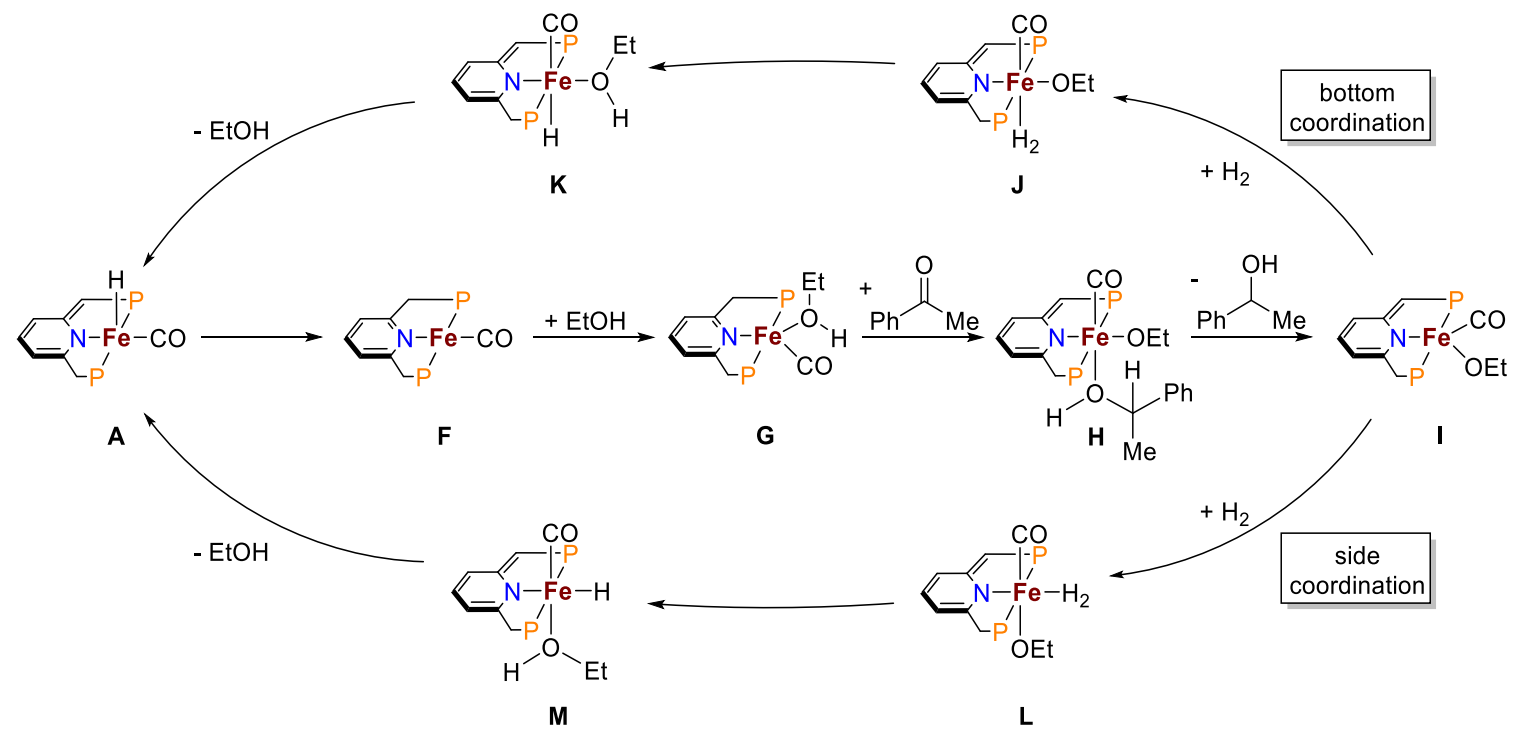

Scheme 18. Computationally evaluated mechanisms for ketone hydrogenation with $\mathrm{Fe}\left(\mathbf{P N}^{\mathbf{P y}} \mathbf{P}\right)$ catalysts. $^{412}$

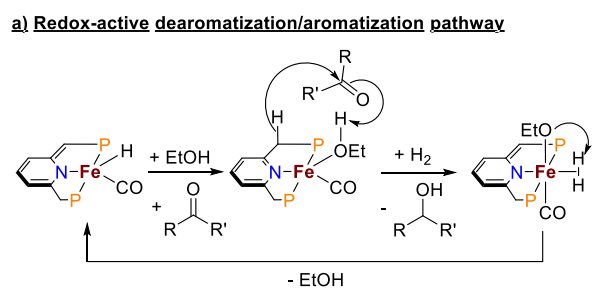

b) Non-redox monohvdride pathway

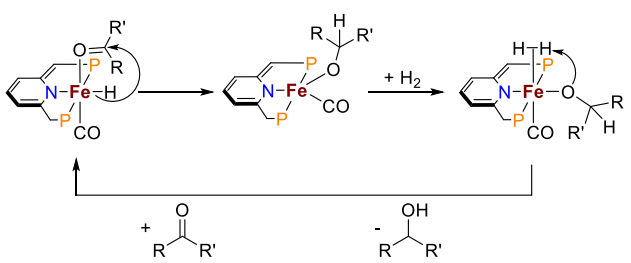

c) Non-redox dihvdride pathwav without dearomatization/aromatization

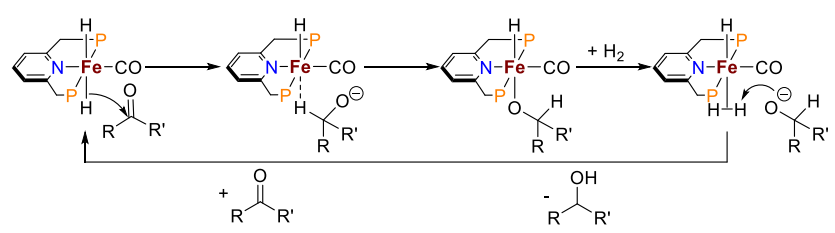

d) Non-redox dihvdride pathwav with dearomatization/aromatization

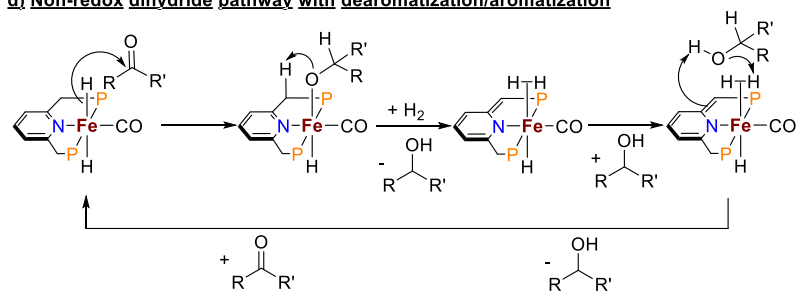

Milstein and coworkers reported the synthesis of precatalyst Fe-21, with an unsymmetrical PNN pincer ligand (Scheme 19). ${ }^{413}$ Acetophenone and some mostly aliphatic ketones are reduced with low catalyst loadings and pressures. The relatively high loadings of the strong base $\mathrm{KN}\left(\mathrm{SiMe}_{3}\right)_{2}$ required the use of THF as solvent. A strong dependence of the TON on the base/catalyst ratio was reported.

Scheme 19. Acetophenone hydrogenation with precatalyst Fe21. ${ }^{413}$
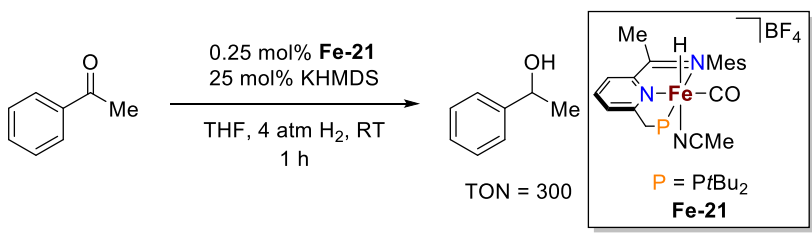

Precatalyst Fe-22a (Scheme 20) was investigated in the catalytic hydrogenation of ketones. ${ }^{414}$ Similar substrate scope, yet lower activity was obtained in comparison to Fe-1c. While acetophenone was hydrogenated quantitatively $(0.5$ mol\% Fe-22a; 1 mol\% $\%$ OtBu; 5 atm $\mathrm{H}_{2}$; EtOH; r.t.), low yields were obtained for aliphatic and $\alpha, \beta$-unsaturated ketones. No catalytic activity in ketone hydrogenation was obtained for the analogous $N$-methylated complex, suggesting the relevance of metal-ligand cooperativity.

Scheme 20. Ketone hydrogenation with catalyst Fe-22a. a: Yields were determined by NMR spectroscopy. ${ }^{414}$

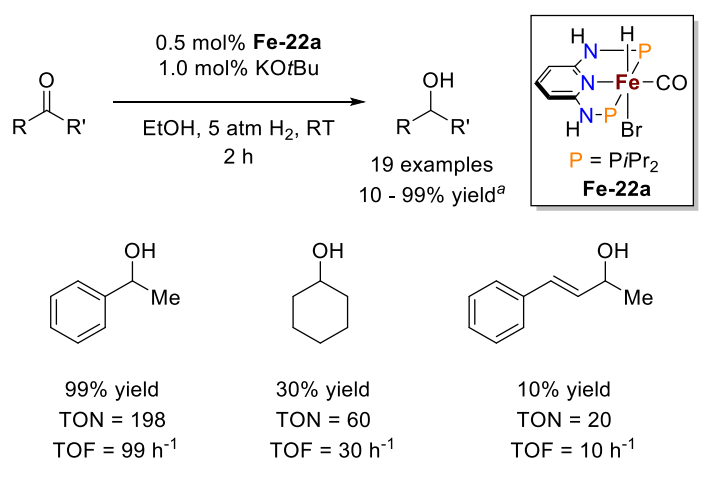


Stoichiometric control experiments in aprotic solvents revealed that formation of the in situ generated cis- and transdihydrides do not react with $\mathrm{PhC}(\mathrm{O}) \mathrm{Me}$. Furthermore, the dihydrides are not formed in $\mathrm{EtOH}$, highlighting the role of the solvent in stabilizing the dearomatized state. Based on these findings, an inner-sphere all-iron(II) monohydride pathway was proposed (Scheme 21, a) ${ }^{414}$ However, Morello and Hopmann computed a high effective barrier for this mechanism $\left(32.7 \mathrm{kcal} \cdot \mathrm{mol}^{-1}\right.$ for $\left.\mathrm{PhC}(\mathrm{O}) \mathrm{Me}\right)$ and favored a dihydride mechanism (Scheme 21, b) with much lower effective barrier $\left(20.2 \mathrm{kcal} \cdot \mathrm{mol}^{-1}\right){ }^{412}$ As for the $\mathbf{P N}{ }^{\mathbf{P y}} \mathbf{P}$ catalyst, acetophenone insertion into a $\mathrm{Fe}-\mathrm{H}$ bond was computed to be slightly endergonic, in line with the stoichiometric control experiments in both cases. ${ }^{409,414}$

Scheme 21. Computed pathways for ketone hydrogenation catalyzed by the $\mathrm{Fe}\left(\mathbf{P N}_{3} \mathbf{P}\right)$ platform. ${ }^{414}$

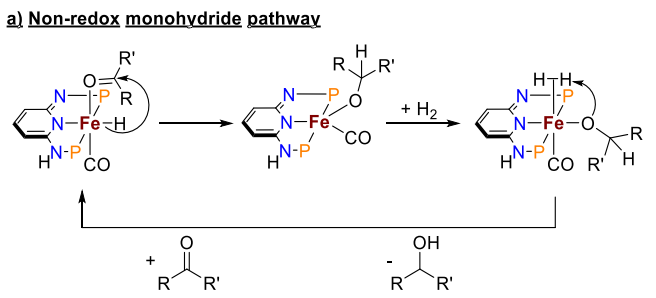

b) Non-redox dihvdride pathwav with dearomatization/aromatization

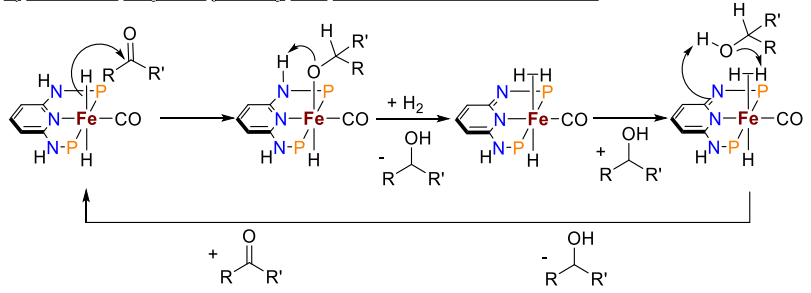

A modified $\mathrm{PN}_{3} \mathrm{P}$ framework with axially chiral phosphoryl substituents were reported by Kirchner's group (Scheme 22). The iron precatalyst $\mathbf{F e - 2 2 b}$ was directly used as an inseparable mixture of two diastereomers in the hydrogenation of acetophenones with good activity at mild conditions. ${ }^{415}$

Scheme 22. Hydrogenation of acetophenones with precatalyst Fe22b. Yields were determined by ${ }^{1} \mathrm{H}$ NMR spectroscopy. ${ }^{415}$<smiles>[X]c1ccc(C(C)=O)cc1</smiles>

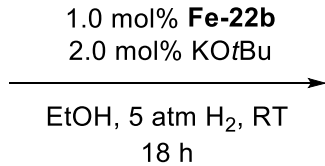

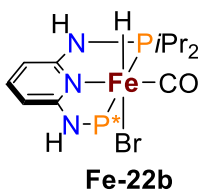<smiles>CP1Cc2ccc3ccccc3c2-c2c(ccc3ccccc23)C1</smiles>

In comparison to ketones, aldehyde hydrogenation with precatalyst Fe-22a exhibits low TON. Full conversion of aromatic and secondary aliphatic aldehydes was obtained with $5 \mathrm{~mol} \%$ catalyst loading and good TOF $\left(\mathrm{ca} .100 \mathrm{~h}^{-1}\right.$ ) at mild reaction conditions (5 atm $\mathrm{H}_{2}$, r.t., $<20$ min; Table 2). ${ }^{414} \mathrm{In}$ contrast to picolinaldehyde, isonicotinaldehyde hydrogenation was inhibited by substrate coordination to the catalyst.

Table 2. Hydrogenation of aldehydes with catalyst Fe-22a. ${ }^{414}$

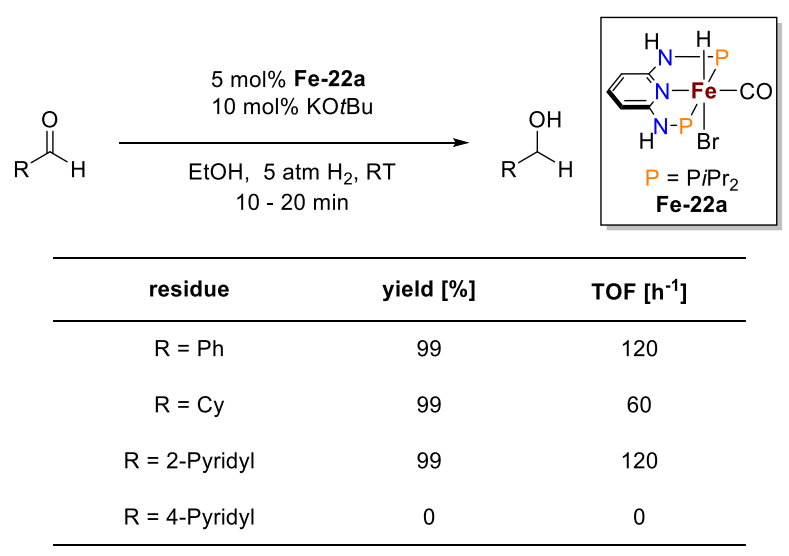

In a subsequent study, Gorgas et al. investigated the backbone $N$-methylated complex Fe-22Me. ${ }^{416}$ This precatalyst exhibits exceptionally high activity in the hydrogenation of 4-fluorobenzaldehyde $\left(\mathrm{TON}_{\mathrm{Max}}=80000 ; \mathrm{TOF}_{\mathrm{Max}}=20000\right.$ $\mathrm{h}^{-1}$ ), exceeding the activity of parent Fe-22a by several orders of magnitude (Scheme 23). Another interesting feature is the high chemoselectivity with respect to aldehyde reduction $v s$. ketones, esters and olefins. Since a metal-ligand cooperative pathway is not possible, the authors proposed a dihydride mechanism for this catalyst.

In their comprehensive computational study, Morello and Hopmann examined the origin of the exceptional chemoselectivity of Fe-22Me in comparison with Fe-1c and Fe-22a, respectively. ${ }^{412}$ As proposed by Kirchner, the data support a dihydride mechanism without de-/aromatization for $\mathbf{F e}-$ 22Me (Scheme18, c) in contrast to Fe-1c and Fe-22a (pathway d). The shift in mechanism is associated with different turnover determining intermediates (TDI) and transition states (TDTS). ${ }^{417}$ In case of pathway d, the TDI (iron(II) alkoxide state) and TDTS $\left(\mathrm{H}_{2}\right.$ heterolysis) exhibit similar substrate dependence, resulting in about the same energetic span for (unselective) hydrogenation of different substrates. In contrast, pathway c features the dihydride resting state as TDI and the $\mathrm{H}_{2}$ binding step as TDTS. It is evident that in this case the TDI is substrate independent while the $\mathrm{Fe}^{+} /$alkoxide ${ }^{-}$ion-separated TDTS correlates with the product $\mathrm{p} K_{\mathrm{a}}$, which results in strong substrate discrimination. Hence, the selectivity is quenched if the resting state and the step with highest kinetic barrier share a linear free energy relationship for different substrates. ${ }^{418}$ Given that the TDTS is often a substrate transformation step, a general guideline to achieve (chemo)selectivity can be to avoid the overstabilization of the substrate-bound ground states. 
Scheme 23. Hydrogenation of 4-Fluorobenzaldehyde with $\mathrm{PN}_{3} \mathrm{P}$ Iron Catalyst Fe-22Me. ${ }^{416}$

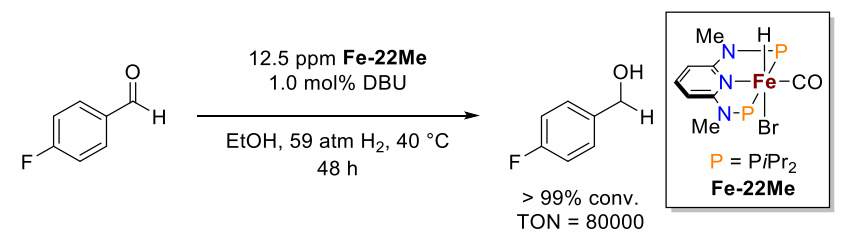

The $\mathbf{P N}_{\mathbf{3}} \mathbf{P}$ pincer ligated manganese tricarbonyl complex Mn-22a was utilized by Sortais and coworkers for the catalytic hydrogenation of ketones (Scheme 24). ${ }^{419}$ Stoichiometric NMR experiments revealed the formation of a manganese(I) hydride in the presence of $\mathrm{H}_{2}$ and base, which requires $\mathrm{CO}$ dissociation, in contrast to Kempe's previously reported $\mathbf{P N}_{5} \mathbf{P}$ based dicarbonyl manganese precatalyst (see below). ${ }^{420}$ Accordingly, the catalytic activity is inferior and prolonged reaction times are required for $\mathbf{M n - 2 2 a}$, despite high catalyst loadings, $\mathrm{H}_{2}$ pressures and temperatures.

Scheme 24. Hydrogenation of acetophenone with precatalyst Mn-22a. ${ }^{419}$

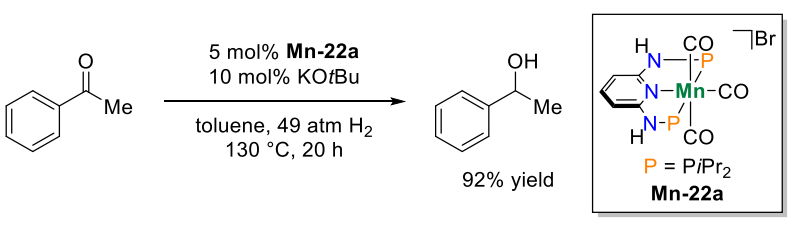

As an alternative, Kirchner and co-workers directly used the manganese dicarbonyl hydride complex Mn-22b as catalyst (Scheme 25). ${ }^{421}$ The authors found pronounced chemoselectivity towards aldehydes. Turnover numbers up to 10400 were achieved for 4-fluorobenzaldehyde hydrogenation. Remarkably low catalyst loadings $(0.05-0.1 \mathrm{~mol} \%)$ can be used to reduce a broad range of aldehydes under 49 atm $\mathrm{H}_{2}$ in ethanol, including $\alpha, \beta$-unsaturated and aliphatic aldehydes.

Scheme 25. Hydrogenation of aldehydes with precatalyst Mn-22b (a: Yields determined by ${ }^{1} \mathrm{H}$ NMR spectroscopy). ${ }^{421}$

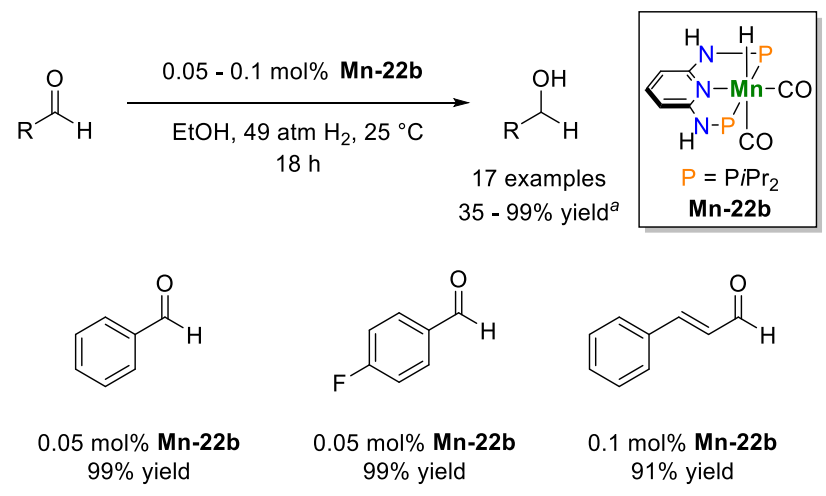

Stoichiometric experiments revealed, that $\mathbf{M n - 2 2 b}$ reacts with aldehydes and not with 4-fluoroacetophenone in both protic and nonprotic solvents. The alkoxide products do not undergo hydrogenolysis. The two main mechanisms that were discussed for the iron pincer platforms, i.e. outersphere substrate insertion followed by $\mathrm{H}_{2}$ heterolysis either with (Scheme 18, d) or without (Scheme 18, c) pincer ligand cooperativity, were also evaluated computationally for Mn22b (Scheme 26). ${ }^{421}$ Almost identical overall effective barriers $\left(\Delta \Delta G^{*}=1 \mathrm{kcal} \mathrm{mol}^{-1}\right)$ were obtained for the two scenarios. The alkoxide represents the TDI for both routes, while the substrate is only involved in the TDTS of the non-MLC route. The MLC-pathway might therefore be better suited to explain the high chemoselectivity for aldehydes vs. ketones, which should be addressed in future studies. Notably, the respective manganese hydrides with $\mathrm{NMe}$ and $\mathrm{CH}_{2}$ backbone linkers showed no (NMe) or poor $\left(\mathrm{CH}_{2}\right)$ activity further supporting a metal-ligand cooperative mechanism.

Scheme 26. Proposed Mechanism for the aldehyde hydrogenation with precatalyst $\mathbf{M n - 2 2 b}\left(\mathrm{P}=\mathrm{PiPr}_{2}\right){ }^{421}$

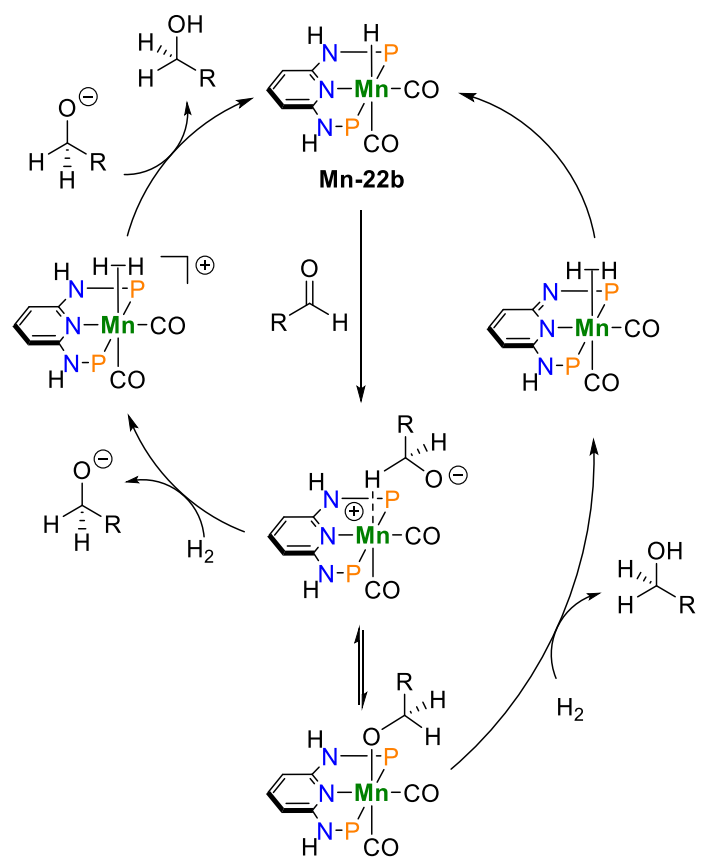

Kempe and coworkers introduced the $\mathbf{P N}_{5} \mathbf{P}$ pincer ligand platform with a triazine core for manganese catalyzed hydrogenation (Scheme 27). ${ }^{420}$ The manganese dicarbonyl complex Mn-23 is a highly efficient catalyst for the hydrogenation of a vast range of aromatic and aliphatic ketones at milder reaction conditions compared with Beller's aliphatic precatalyst Mn-15a (Scheme 27). Benzaldehyde is quantitatively hydrogenated at mild conditions $(0.1 \mathrm{~mol} \% \mathbf{M n}-23$, $40{ }^{\circ} \mathrm{C}$ ). 
Scheme 27. Hydrogenation of Ketones with the precatalyst Mn-23 (a: Yields determined by GC and isolation). ${ }^{420}$

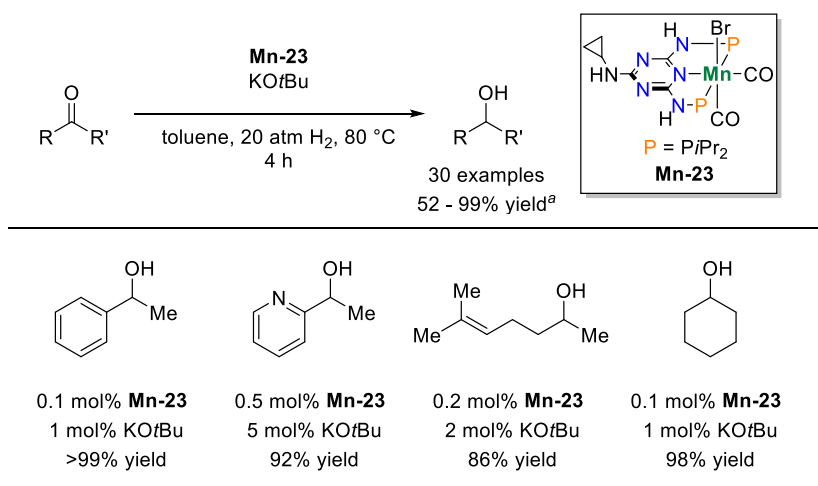

The same group employed the $\mathbf{P N} \mathbf{5} \mathbf{P}$ cobalt dichloride complex Co-23a as precatalyst for the catalytic hydrogenation of ketones and aldehydes (Scheme 28). ${ }^{422} 24$ ketones and aldehydes were reduced in predominantly very good yields applying $20 \mathrm{~atm} \mathrm{H}_{2}$ at room temperature with catalyst loadings ranging from $0.25 \mathrm{~mol} \%$ to $3 \mathrm{~mol} \%$. Conjugated and remote $\mathrm{C}=\mathrm{C}$ double bonds were retained in the catalytic experiments, which reflects the inverse selectivity in comparison to the aliphatic amino pincer catalyst reported by Hanson and coworkers (see below). ${ }^{195}$ The authors propose the formation of a coordinatively unsaturated active cobalt(II) catalyst with a dianionic pincer ligand upon activation of Co23a with two equivalents of $\mathrm{NaO} t \mathrm{Bu}$, from trapping with bipyridine.

Scheme 28. Hydrogenation of ketones and aldehydes with precatalyst Co-23a (a: Yields determined by GC). ${ }^{422}$

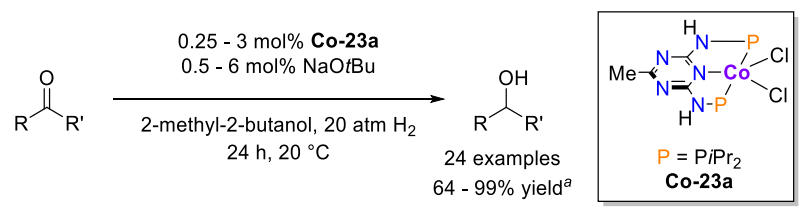

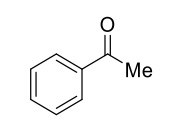

$0.25 \mathrm{~mol} \%$ Co-23a $0.5 \mathrm{~mol} \% \mathrm{NaOtBu}$ $99 \%$ yield

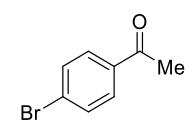

$2 \mathrm{~mol} \% \mathrm{Co}-23 \mathrm{a}$ $2 \mathrm{~mol} \% \mathrm{Co}-23 \mathrm{a}$
$4 \mathrm{~mol} \% \mathrm{NaOtBu}$ $4 \mathrm{~mol} \% \mathrm{NaOtBu}$
$64 \%$ yield
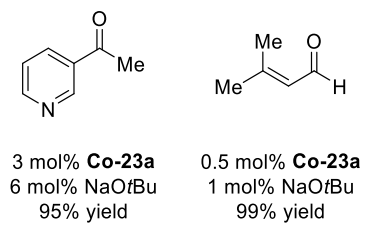

3.1.2. Dialkylamino Pincer Catalysts Morris and co-workers introduced several iron precatalysts with aliphatic PNP pincer ligands for the catalytic hydrogenation of ketones with low catalyst loadings $(0.2 \mathrm{~mol} \%)$ in THF (Table 3 ), including asymmetric hydrogenation (see below). ${ }^{423}$

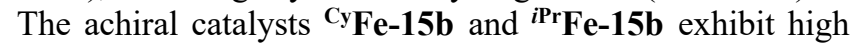
activities in acetophenone hydrogenation (TOF $=1980 \mathrm{~h}^{-1}$ ) at $50{ }^{\circ} \mathrm{C}$ and good activity at r.t. for precatalyst ${ }^{i \text { Pr }} \mathbf{F e}-\mathbf{1 5 b}$ $\left(\mathrm{TOF}=990 \mathrm{~h}^{-1}\right)$. Activation of the imine precatalysts requires addition of lithium aluminium hydride (LAH) and tert-amyl alcohol, respectively. A stoichiometric control experiment with excess LAH indicated the formation of an
iron(II) monocarbonyl hydride complex, $\left[\mathrm{FeH}(\mathrm{CO})\left\{\mathrm{H}_{3} \mathrm{AlN}\left(\mathrm{CH}_{2} \mathrm{CH}_{2} \mathrm{PCy}_{2}\right)\left(\mathrm{CH}_{2} \mathrm{CH}_{2} \mathrm{PPh}_{2}\right)\right\}\right]$, i.e. an alane adduct of the hydride amide catalysts directly used by other groups (see below). Accordingly, alcoholylsis produces an alkoxide hydride complex that is also an intermediate in the proposed catalytic cycles.

Table 3. (Asymmetric) Hydrogenation of ketones with the precatalysts ${ }^{\mathrm{Cy}} \mathbf{F e}-15 \mathrm{~b},{ }^{\mathrm{PP}} \mathrm{Fe}-15 \mathrm{~b},{ }^{\mathrm{Ph}, \mathrm{PP}} \mathrm{Fe}-15 \mathrm{~b}$ and $\mathbf{F e - 1 5 c}$ (deviation from standard conditions: a: $0.1 \mathrm{~mol} \% \mathbf{F e}-15 \mathrm{c}, 1.0 \mathrm{~mol} \% \mathrm{KO} t \mathrm{Bu}$, 6 eq. $\mathrm{LiAlH}_{4}$; b: $0.1 \mathrm{~mol} \%{ }^{\mathrm{Ph}, i \mathbf{P r}}{ }_{\mathrm{Fe}-15 \mathrm{~b}}, 1.0 \mathrm{~mol} \% \mathrm{KO} t \mathrm{Bu}$, xs. $\left.\mathrm{LiAlH}_{4}, 10 \mathrm{~atm} \mathrm{H}, t \mathrm{AmOH} / \mathrm{THF}\right) .{ }^{423}$

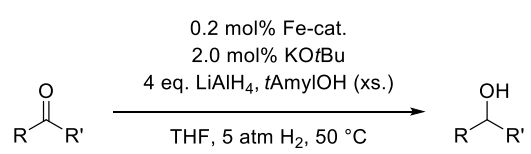

\begin{tabular}{|c|c|c|c|c|c|c|}
\hline substrate & catalyst & $t$ [min] & conv. [\%] & ee [\%] & TON & TOF $\left[h^{-1}\right]$ \\
\hline & ${ }^{c y} \mathrm{Fe}-15 b$ & 15 & 99 & - & 495 & 1980 \\
\hline & ${ }^{i P r} \mathrm{Fe}-15 b$ & 15 & 99 & - & 495 & 1980 \\
\hline & $\mathrm{Fe}-15 \mathrm{c}^{a}$ & 30 & 99 & $80(S)$ & 990 & 1980 \\
\hline & $\mathrm{Fe}-15 c^{a}$ & 1440 & 0 & $\mathrm{n} / \mathrm{a}$ & 0 & 0 \\
\hline & $\mathrm{Fe}-15 c^{a}$ & 240 & 95 & 0 & 950 & 240 \\
\hline & $\mathrm{Fe}-15 c^{a}$ & 48 & 99 & $85(S)$ & 990 & 1230 \\
\hline & $\begin{array}{c}\mathrm{Ph}, \mathbf{P P r} \mathrm{Fe}- \\
15 \mathbf{b}^{b}\end{array}$ & 150 & 90 & - & 900 & 360 \\
\hline
\end{tabular}
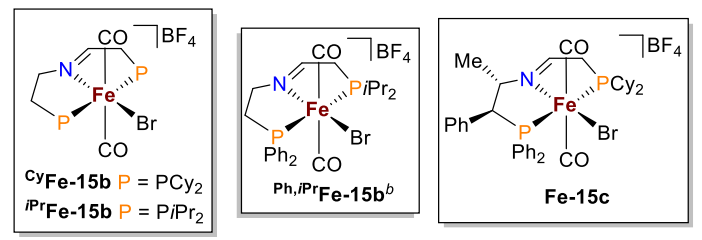

Benzaldehyde hydrogenation with Fe-15c was obtained with $90 \%$ conversion within 2.5 hours (Table 3 ). Considerably higher dihydrogen pressures (10 atm) are required compared with pyridyl based pincer catalysts (see above). ${ }^{423}$

Jones, Schneider and coworkers reported the hydrogenation of ketones using the aliphatic iron PNP pincer catalyst ${ }^{i \text { Pr }} \mathbf{F e}$ 15a at mild, base-free conditions in THF at 10 atm $\mathrm{H}_{2}$ (Scheme 29). ${ }^{424}$ In comparison, catalytic reactions with pyridine-based catalysts Fe-1c and Fe-1d are usually carried out in alcoholic solvents (see above). High $\mathrm{C}=\mathrm{O}$ chemoselectivity was obtained for the $\alpha, \beta$-unsaturated ketone benzalacetone to the kinetic allyl alcohol product. Interestingly, the Jones group later reported the full hydrogenation of benzalacetone with the same catalyst in benzene, at higher catalyst loadings ( $5 \mathrm{~mol} \%$ ) and lower $\mathrm{H}_{2}$ pressure (Scheme 72 , section 3.7.2). NMR monitoring verified the allylic alcohol as the main intermediate. The apparent $\mathrm{H}_{2}$ pressure dependence of the selectivity suggests that the full hydrogenation of benzalacetone might proceed via allyl alcohol isomerization 

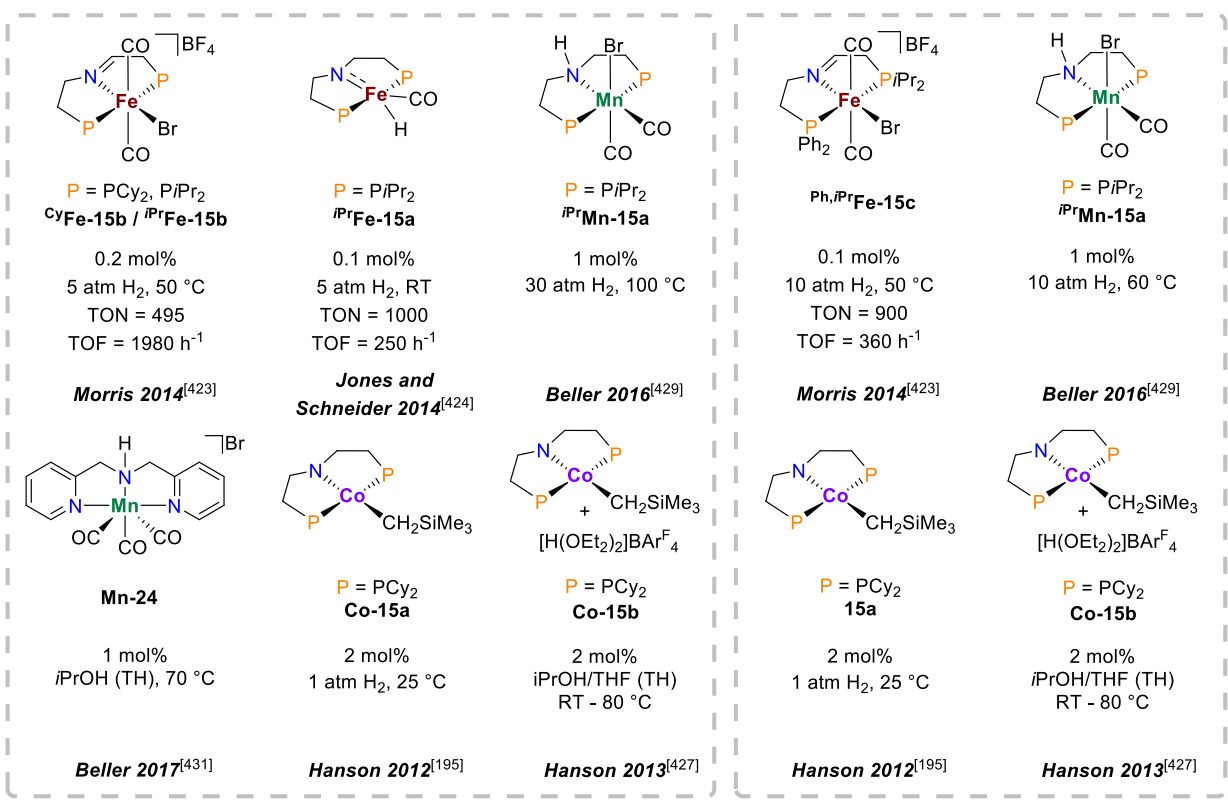

Figure 30. Ketone and aldehyde hydrogenation precatalysts with dialkylamino PNP and NNN pincer ligands.

( $\mathrm{C}=\mathrm{O}$ dehydrogenation $/ \mathrm{C}=\mathrm{C}$ hydrogenation $)$, which requires the amide catalyst state.

Scheme 29. Ketone hydrogenation catalyzed by complex ${ }^{i P r} \mathbf{F e}-$ $15 a^{424}$

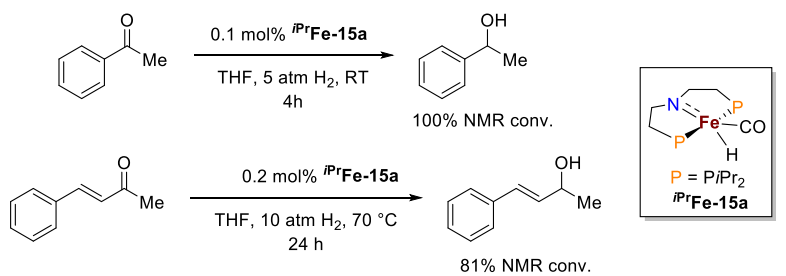

Accordingly, in the absence of $\mathrm{H}_{2}$ the isomerization of allylic alcohols to ketones is catalyzed by the $\mathrm{Fe}\left({ }^{i \operatorname{Pr}} \mathbf{P N}{ }^{\mathbf{H}} \mathbf{P}\right)$ catalyst platform (Scheme 30$).{ }^{425}$ A broad range of allylic alcohols were isomerized to the respective ketone with precatalyst $\mathbf{F e - 1 5 d} / \mathrm{KO} t \mathrm{Bu}$. In 2-propanol the fully reduced transfer hydrogenation product was mainly obtained. A 'self-hydrogen-borrowing' mechanism was proposed based on control experiments and DFT calculations (Scheme 30), which is discussed in detail in Section 5. After AAD of the allylic alcohol the $\alpha, \beta$-unsaturated ketone is rehydrogenated either by 1,4-addition or 1,2-addition, which both give the ketone after vinylalcohol tautomerization. 1,4- and 1,2-addition exhibited similar kinetic barriers by DFT.
Scheme 30. (a) Catalytic Isomerization of Allylic Alcohols with Iron PNP Pincer Complex Fe-15d. (b) Proposed Self-Hydrogen Borrowing Mechanism for the Isomerization of Allylic Alcohols Catalyzed by Iron Pincer Complexes $\left(\mathrm{P}={\left.\mathrm{P} i \mathrm{Pr}_{2}\right) .}^{425}\right.$

A)

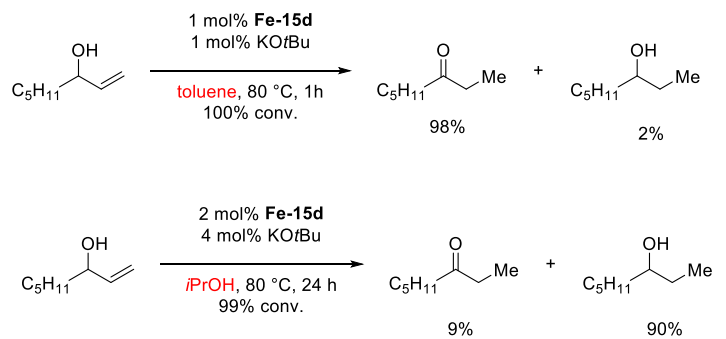

B)

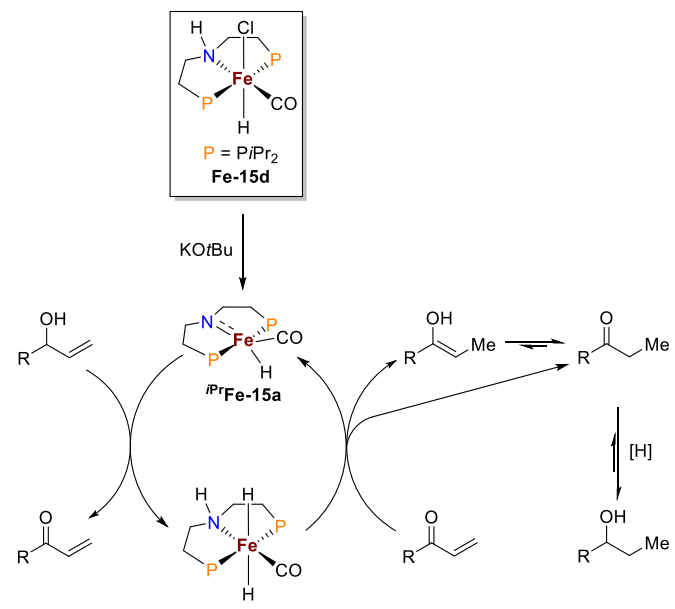

Hanson and coworkers established a versatile cobalt catalyst for the hydrogenation of $\mathrm{C}=\mathrm{C}, \mathrm{C}=\mathrm{O}$ and $\mathrm{C}=\mathrm{N}$ double bonds at mild conditions, which is discussed in detail in Sections 3.7.1. and 3.2, respectively. The square-planar, low-spin cobalt(II) pincer precatalyst Co-7 was used, which requires activation with Brookhart's acid $\left[\mathrm{H}\left(\mathrm{Et}_{2} \mathrm{O}\right)_{2}\right] \mathrm{BAr}_{4}{ }_{4}$ (Scheme 
31). ${ }^{195}$ The activated precatalyst was crystallographically characterized. However, the structural features shed doubt on the assignment as the $N$-protonated complex [ $\left[{ }^{{ }^{\mathbf{C}} \mathbf{P}-}\right.$ $\left.\left.\mathbf{N}^{\mathrm{H}} \mathbf{P}\right) \mathrm{Co}^{\mathrm{II}}\left(\mathrm{CH}_{2} \mathrm{SiMe}_{3}\right)\right] \mathrm{BAr}_{4}{ }_{4}(\mathbf{C o}-15 \mathbf{b}) .{ }^{195}$ The almost planar coordination environment around the nitrogen donor and alternating $\mathrm{C}-\mathrm{N}$ bond lengths $(1.38 / 1.45 \AA)$ in the pincer backbone suggest formation of an imine species upon protonation. Interestingly, the corresponding tetraphenylborate salt (from protonation of Co-15a with $\left[\mathrm{HNEt}_{3}\right] \mathrm{BPh}_{4}$ ) features different backbone metrics (1.48/1.50 $\AA$ ) and exhibits considerably lower activity in acetophenone hydrogenation. ${ }^{426}$ The ambiguity regarding the activated precatalyst species requires further clarification.

Scheme 31. Hydrogenation of C-O, C-C and C-N double bonds with precatalyst Co-15a. ${ }^{195}$
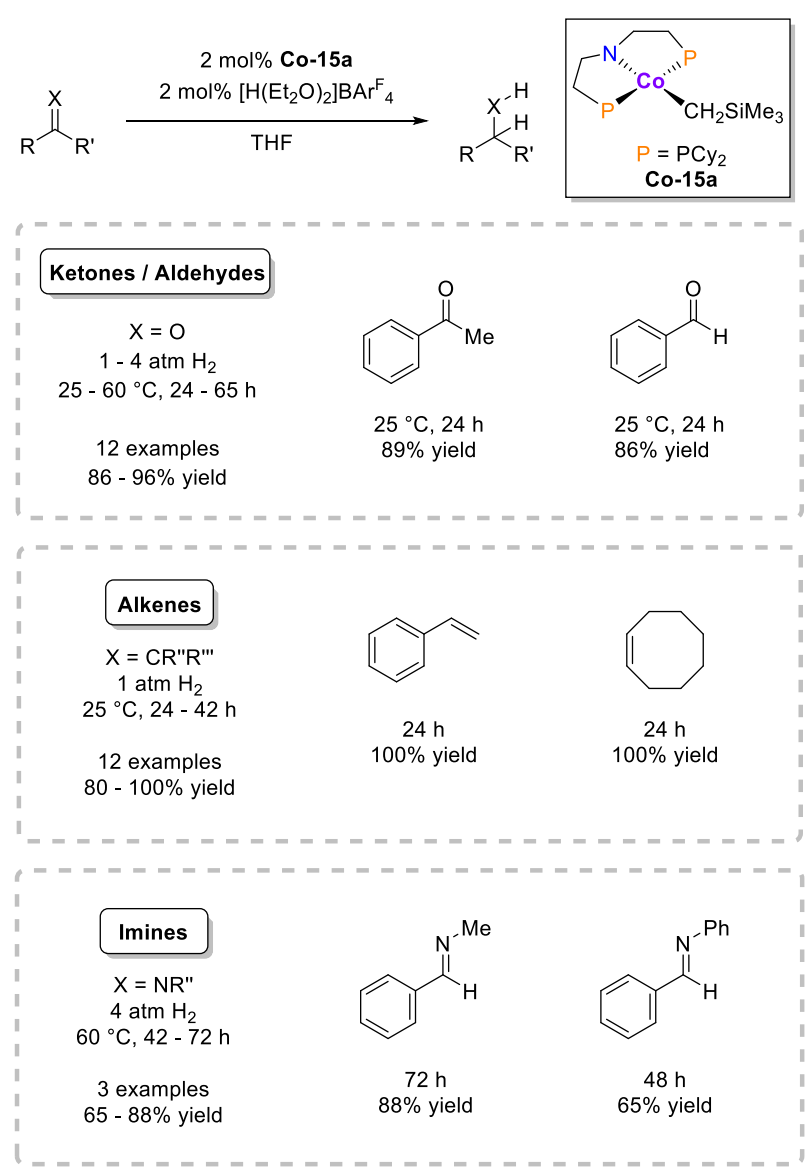

The high activities for both unactivated olefins and carbonyl groups are rather unusual for catalysts with this ligand. In comparison, the iron(II) amide catalyst Fe-15a generally exhibits $\mathrm{C}=\mathrm{O}$ over $\mathrm{C}=\mathrm{C}$ chemoselectivity and preferentially reduces activated, electron deficient olefins. ${ }^{196}$ For the iron catalyst, this is attributed to an outer-sphere mechanism, as discussed above and in Section 3.7. In contrast, cobalt catalyzed olefin hydrogenation was proposed to operate via inner-sphere olefin insertion at the cobalt(II) hydride [ $\left({ }^{\mathbf{C y}} \mathbf{P}\right.$ $\left.\left.\mathbf{N}^{\mathbf{H}} \mathbf{P}\right) \mathrm{CoH}\right]^{+}$, which is discussed in Section 3.7.2. ${ }^{195,426}$ For ketone hydrogenation, the redox states of the active cobalt species remain unclear. From reverse, acceptorless alcohol dehydrogenation, a cobalt(III) complex could be isolated but it exhibits much lower catalytic activity in ketone hydrogenation than parent $\mathbf{C o - 1 5 a} /\left[\mathrm{H}\left(\mathrm{Et}_{2} \mathrm{O}\right)_{2}\right] \mathrm{BAr}^{\mathrm{F}}{ }_{4}{ }^{426}$ Furthermore, the $N$-methylated precatalyst $\left[\left({ }^{\mathbf{C}_{\mathbf{y}} \mathbf{P}-}\right.\right.$ $\left.\left.\mathbf{N}^{\mathrm{Me}} \mathbf{P}\right) \mathrm{Co}^{\mathrm{II}}\left(\mathrm{CH}_{2} \mathrm{SiMe}_{3}\right)\right] \mathrm{BAr}{ }_{4}(\mathbf{C o - 1 5 M e})$ is not active in acetophenone hydrogenation suggesting the relevance of MLC.

Hanson's catalyst was also used as catalyst for transfer hydrogenation of C-O and C-N double bonds. ${ }^{427}$ With iso-propanol/THF as solvent and hydrogen source, 13 ketones and aldehydes and 3 imines were reduced under mild conditions (2 mol\% @ $25{ }^{\circ} \mathrm{C}$ for $24 \mathrm{~h}$; Imines: $80{ }^{\circ} \mathrm{C}$ ). Deuteration experiments favor a monohydride over dihydride mechanism. ${ }^{428}$ The $N$-methylated pincer complex Co-15Me exhibits only slightly reduced activity in acetophenone hydrogenation, suggesting that MLC is irrelevant for catalysis. Furthermore, the lack of chemoselectivity for transfer hydrogenation of $\alpha, \beta$-unsaturated substrates contrasts with related iron-systems, and was rationalized with an inner-sphere mechanism for the cobalt catalyst.

Scheme 32. Hydrogenation of ketones, aldehydes and nitriles with

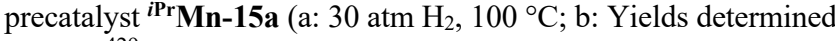
by GC). ${ }^{429}$

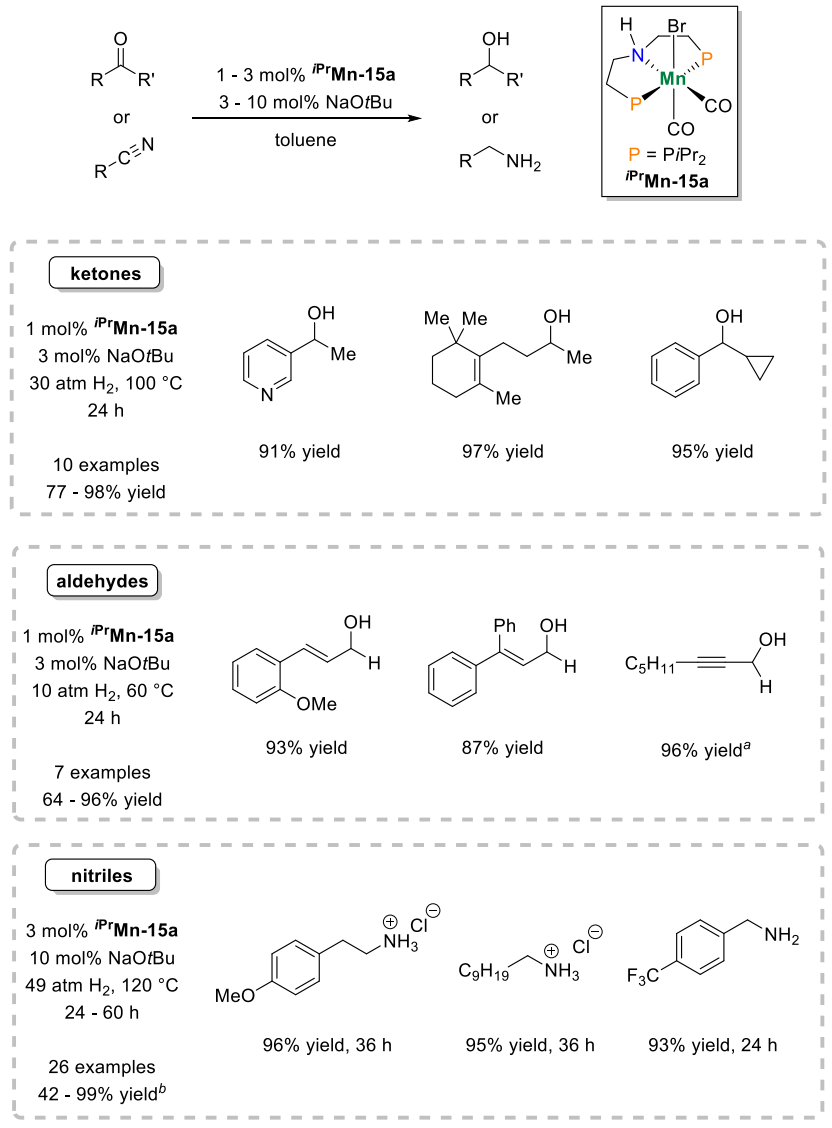

The Beller group introduced ${ }^{i \mathbf{P r}} \mathbf{M n}-\mathbf{1 5 a}$ (Scheme 32) as the first manganese precatalyst for the hydrogenation of polar multiple bonds. ${ }^{429}$ A broad range of aromatic and aliphatic ketones, aldehydes and nitriles are hydrogenated in moderate to quantitative yields using 1-3 mol\% catalyst and 3-10 $\mathrm{mol} \%$ base $(\mathrm{NaO} t \mathrm{Bu})$ loadings under harsher conditions (10$49 \mathrm{~atm} \mathrm{H}_{2} ; 60-100{ }^{\circ} \mathrm{C} ; 24-60$ hours) as compared with the 


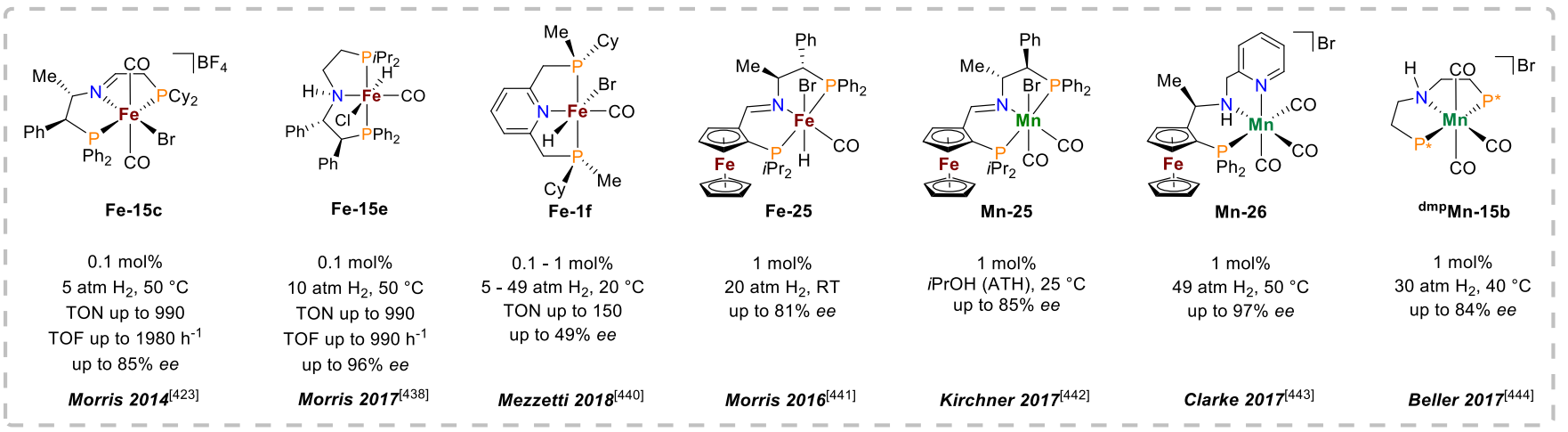

Figure 31. Summary of asymmetric ketone hydrogenation catalysts based on tridentate PNP and PNN Ligands $(\mathrm{P} *=\mathrm{Pdmp} ; \mathrm{dmp}=(2 R, 5 R)-$ 2,5-dimethylphospholane).

analogous iron system. Unpolar $(\mathrm{C}=\mathrm{C})$ double bonds are tolerated, as well as potentially coordinating substrates like 3acetylpyridine. The almost quantitative yield of the cyclopropyl-substituted ketone precludes a reaction mechanism that proceeds via stable radical intermediates.

${ }^{1} \mathrm{H}$ NMR and IR spectroscopic examination revealed hydride formation trans-positioned to a $\mathrm{CO}$ ligand upon reaction of ${ }^{i P r} \mathrm{Mn}-15 \mathrm{a}$ with dihydrogen and $\mathrm{NaO} t \mathrm{Bu}$. In analogy to related iron-systems the catalytically active species are proposed to be manganese amine-hydride and amide complexes. DFT computations indicated almost thermoneutral $\mathrm{H}_{2}$ 1,2-addition to the amide ( $\left.{ }^{i \mathbf{P r}} \mathbf{P N P}\right) \mathrm{Mn}(\mathrm{CO})_{2}$. A related catalyst was described by Sortais and coworkers for the hydrogenation of ketones. ${ }^{430}$ In contrast to the corresponding rhenium system, the manganese bis(phosphinite) pincer precatalyst ( $1 \mathrm{~mol} \%$ ) exhibits $25 \%$ conversion in acetophenone hydrogenation. Interestingly, the backbone methylated analogous complex showed only slightly lower conversion $(20 \%)$.

Scheme 33. Transfer hydrogenation of acetophenone with precatalyst Mn-24 (a: Yield determined by GC). ${ }^{431}$

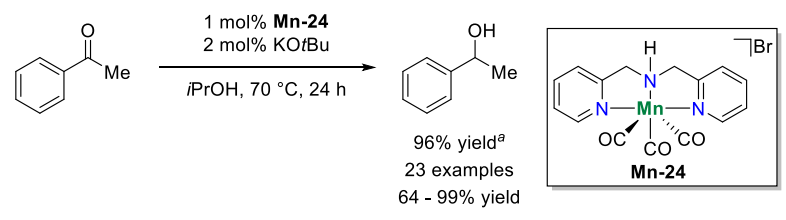

Beller and co-workers also reported catalytic transfer hydrogenation of ketones with Mn dipyridyl amine pincer complex Mn-24 (Scheme 33). ${ }^{431} i \mathrm{PrOH}$ was used as hydrogen source at $80{ }^{\circ} \mathrm{C}$ over the course of 24 hours with $1 \mathrm{~mol} \%$ catalyst loading and $2 \mathrm{~mol} \%$ base $(\mathrm{KO} t \mathrm{Bu})$. A broad range of ketones was hydrogenated in moderate to almost quantitative yields. The authors found comparable catalytic activity with the analogous $N$-methylated backbone precursor, suggesting that pincer-mediated, metal-ligand cooperative $\mathrm{H}_{2}$-activation is not a general prerequisite.

In summary, iron pincer catalysts are more active than manganese and significantly more active than cobalt pincer catalysts in the hydrogenation of ketones and aldehydes with the same or similar ligand platform (Figure 30). Catalysis in the low digit ppm range is currently only possible with iron pincer catalysts at mild reactions conditions. However, the pool of manganese pincer catalysts is rapily growing.

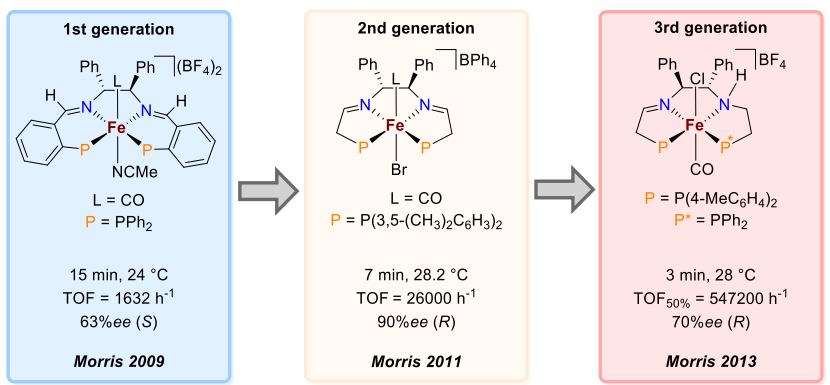

Figure 32. Asymmetric transferhydrogenation of acetophenone with tetradentate PNNP Fe(II) catalysts. ${ }^{432-434}$

\subsubsection{Asymmetric Hydrogenation of Ketones.}

Seminal contributions to iron catalyzed asymmetric transfer hydrogenation (ATH) were made by the Morris group using tetradentate PNNP ligands that are closely related to aforementioned pincer platforms. ${ }^{77}$ Three catalyst generations were reported based on rational ligand design (Figure 32). First generation catalysts with rigid ligand backbone exhibit high TOF $\left(1632 \mathrm{~h}^{-1}\right)$ and medium enantioselectivity $(63 \%$ $e e)$ in acetophenone ATH. ${ }^{432}$ The more flexible second generation ligand gave strongly improved TOF $\left(26000 \mathrm{~h}^{-1}\right)$ and enantioselectivity $(90 \%$ ee $){ }^{433}$ while $3^{\text {rd }}$ generation catalysts exhibit extraordinary activities $\left(\mathrm{TOF}=547200 \mathrm{~h}^{-1}\right)$, albeit with reduced selectivity $(70 \%$ ee $) .{ }^{434}$ Apparently the combination of imine and amine donors is mandatory since the respective diamine complexes were inactive in ATH. Mechanistic investigations and ligand variations with respect to the backbone and phosphorus substituents gave additional insight. ${ }^{435-437}$ While considerably more active than iron pincer catalysts, the PNNP systems are prone to reduced stereoselectivity due to racemization of the product alcohol. 
Scheme 34. Asymmetric ketone hydrogenation with precatalyst Fe-15e (a: Conversion and ee determined by chiral GC). ${ }^{438}$

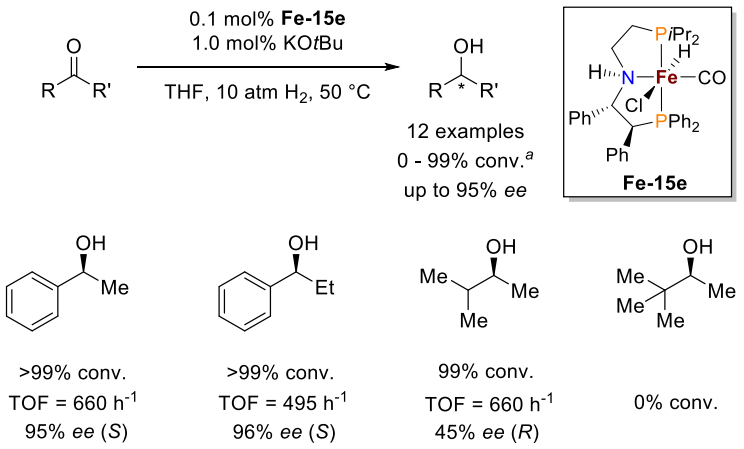
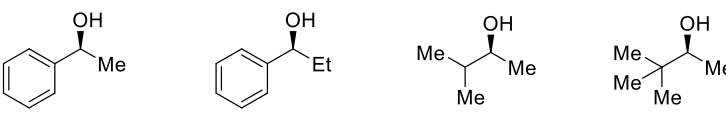

The chiral PNP catalyst Fe-15c (Table 3, Figure 31) was used for asymmetric hydrogenation by the Morris group as an extension of their PNNP-based catalysts. ${ }^{423}$ High catalytic activities and up to $85 \%$ ee were obtained for a range of prochiral ketones. The hydrogenation of a , -unsaturated ketone was chemo- but not stereoselective, while an $\alpha, \beta$-unsaturated ketone was not hydrogenated. The highest enantioselectivities in asymmetric hydrogenation of aromatic ketones with iron pincer catalysts to date were obtained using the second generation catalyst Fe-15e (Scheme 34). ${ }^{438}$ For aliphatic methyl ketones, high conversion but low ee was observed in case of 3-methylbutanone and no conversion for the bulkier substrate 3,3-dimethylbutanone.

Scheme 35. Asymmetric hydrogenation of acetophenone with

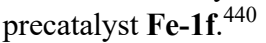

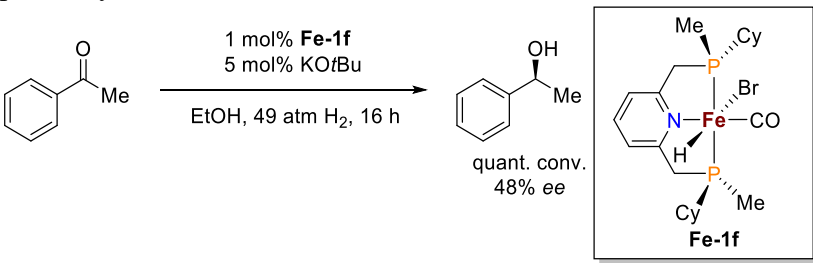

The dependence of activity and stereoselectivity on the ligand backbone substitution pattern was examined by systematic screening of a ligand library. ${ }^{439}$ The highest activities are obtained with $\mathrm{CHMe}$ or $\mathrm{CH}_{2}$ group next to the central nitrogen donor. In turn, the best enantioselectivities are achieved with a $\mathrm{CHPh}$ group next to $\mathrm{PPh}_{2}$ donor, which was rationalized with the subtle interplay of pincer backbone rigidity for stereoselectivity and accessibility of the amine functional group for high reaction rates.

Scheme 36. Asymmetric hydrogenation of acetophenone with precatalyst Fe-25. ${ }^{441}$

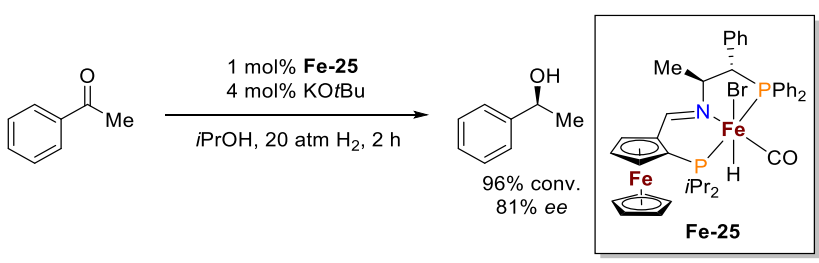

A pyridine-based iron catalyst with chiral phosphine substituents was also reported (Scheme 35). ${ }^{440}$ Precatalyst Fe-1f requires comparatively high pressures and catalyst loadings. Furthermore, low enantioselectivity was found. Importantly, evaluation of the mechanism by means of DFT revealed that only an outer-sphere mechanism with metal-ligand cooperation could reproduce the experimentally observed product stereoselectivity.

Scheme 37. Asymmetric transfer hydrogenation of ketones with precatalyst Mn-25. ${ }^{442}$

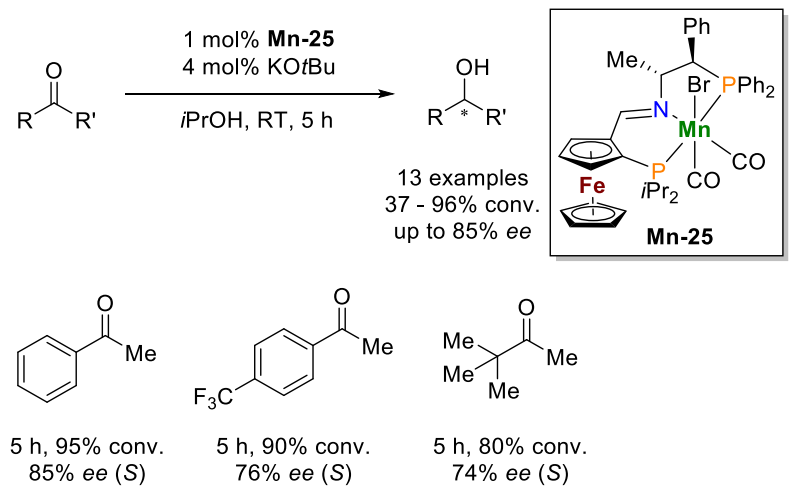

The introduction of a ferrocenyl-bridged pincer backbone was described by Kirchner, Morris and coworkers (Scheme 36). ${ }^{441}$ Enantiomeric excess up to $81 \%$ was obtained at relatively high dihydrogen pressure (20 atm) and catalyst loadings ( $1 \mathrm{~mol} \% \mathbf{F e}-25)$. Kirchner and coworkers also utilized this ligand for manganese-catalyzed ATH of ketones (Scheme 37). ${ }^{442}$ The chiral catalyst Mn-25 gave up to $85 \%$ $e e$ for acetophenone with $i \mathrm{PrOH}$ as hydrogen donor. Addition of gaseous $\mathrm{H}_{2}$ did not affect the yield. Upon reaction of Mn-25 with $\mathrm{KO} t \mathrm{Bu}$ in $i \mathrm{PrOH}$ two isomeric monohydride complexes are formed and proposed as active species. The authors attributed the $S$-selectivity to the steric repulsion and stabilizing hydrogen bonding of the substrate with the pincer backbone.

Clarke and coworkers utilized the tricarbonyl complex Mn26 with a chiral, facially coordinating PNN ligand as the first manganese precatalyst for the asymmetric hydrogenation of ketones (Scheme 38). ${ }^{443}$ A range of 18 aromatic substrates were hydrogenated in moderate to excellent yields. Enantioselectivity showed a strong dependency on the substrate sterics. Enantiomeric excess beyond $80 \%$ was generally obtained with arylketones $\operatorname{ArC}(\mathrm{O}) \mathrm{R}$ that carry secondary and tertiary substituents. High yield but low ee were obtained for ATH with $i \mathrm{PrOH}$ as hydrogen donor. While the mechanism was not explicitly examined, the authors propose MLC, in analogy to Beller's system. 


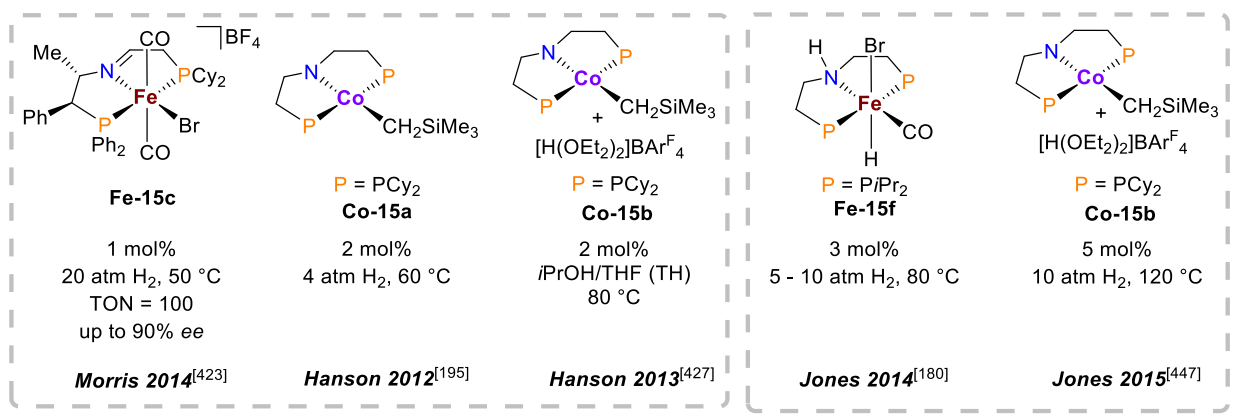

Figure 33. Summary of imine and $N$-heterocycle hydrogenation catalysts based on tridentate PNP Ligands.

Scheme 38. (Asymmetric) hydrogenation of ketones and esters with precatalyst Mn-26. ${ }^{443}$

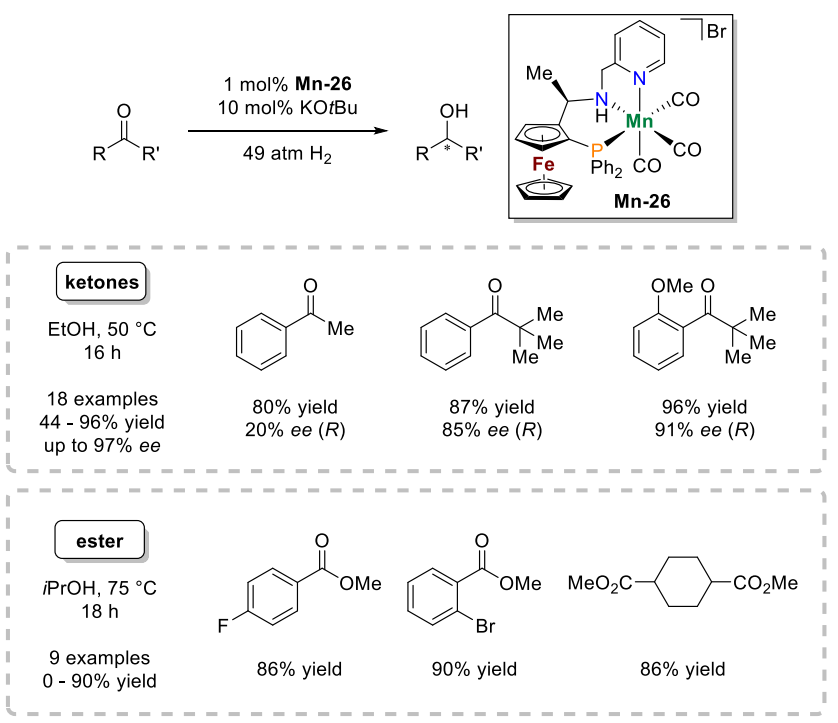

As an extension to their $\left\{\mathrm{Mn}\left(\mathrm{PN}^{\mathrm{H}} \mathrm{P}\right)\right\}$ catalyst platform $\mathbf{M n -}$ 15a, the Beller group examined asymmetric ketone hydrogenation with the chiral catalyst ${ }^{\text {dmp }} \mathbf{M n - 1 5 b}$ (Scheme 39). ${ }^{444}$ In this case, chiral induction is achieved using $(2 R, 5 R)-2,5$ dimethylphospholane substituents. A range of aromatic and also aliphatic ketones were hydrogenated in moderate to good enantioselectivities at $40{ }^{\circ} \mathrm{C}$ and $30 \mathrm{~atm} \mathrm{H}_{2}$ pressure. In contrast to Clark's catalyst Mn-26, this system preferentially catalyzes the hydrogenation of aliphatic ketones with high enantioselectivity.

Scheme 39. Asymmetric hydrogenation of ketones with precatalyst dmp Mn-15b. ${ }^{444}$

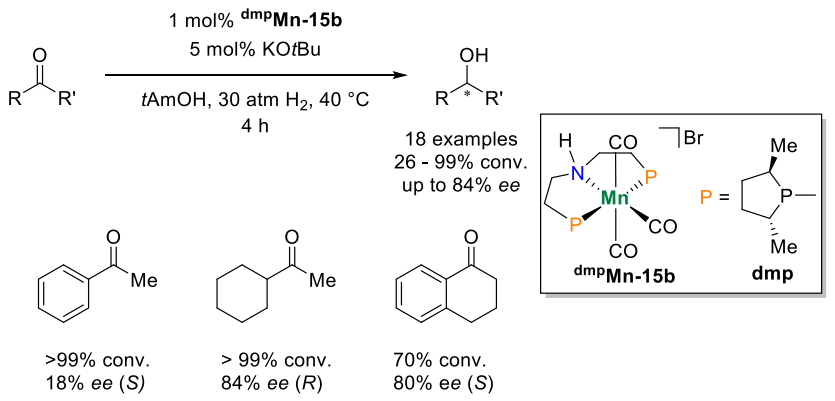

The authors proposed catalyst activation by $\mathrm{CO}$ dissociation and formation of a monohydride-amine catalyst. DFT calculations indicate highly unfavorable dissociation of a second $\mathrm{CO}$ ligand $\left(>42 \mathrm{kcal} \cdot \mathrm{mol}^{-1}\right)$, and facile $\mathrm{H}_{2}$ elimination $\left(\Delta G^{\mathrm{o}}\right.$ $\left.=+1 \mathrm{kcal} \cdot \mathrm{mol}^{-1} ; \Delta G^{*}=20 \mathrm{kcal} \cdot \mathrm{mol}^{-1}\right)$ from a hydride-amine complex, in agreement with the high required pressures. Based on the theoretical model a concerted, outer-sphere MLC-mechanism was proposed via hydride-amine and amide species. The model predicted the correct enantioselectivities but overestimated the barriers for dihydrogen transfer to the substrate $\left(30-36 \mathrm{kcal} \cdot \mathrm{mol}^{-1}\right)$.

\subsection{Hydrogenation of Imines and $N$-Heterocyl- ces}

The chiral complex Fe-15c is an excellent catalyst for the asymmetric hydrogenation of the activated imine $N$-(diphenylphosphanyl)propiophenoneimine (Scheme 40). ${ }^{423}$ The stereoselectivity $(90 \% e e)$ is lower compared with the $\mathrm{Fe}(\mathrm{PNNP})$ catalyst developed by the same group but higher than for an iron catalyst previously reported by Beller and coworkers. $^{445,446}$ Related hydride precatalyst Fe-15f catalyzes the hydrogenation of (iso)quinolines, indoles and pyridines in moderate to good yields at mild conditions (Scheme 40). ${ }^{180}$ A hydride-amine/amide MLC pathway was proposed.

Scheme 40. Hydrogenation of $N$-heterocycles and imines with Iron and cobalt PNP pincer complexes. ${ }^{180,195,423}$

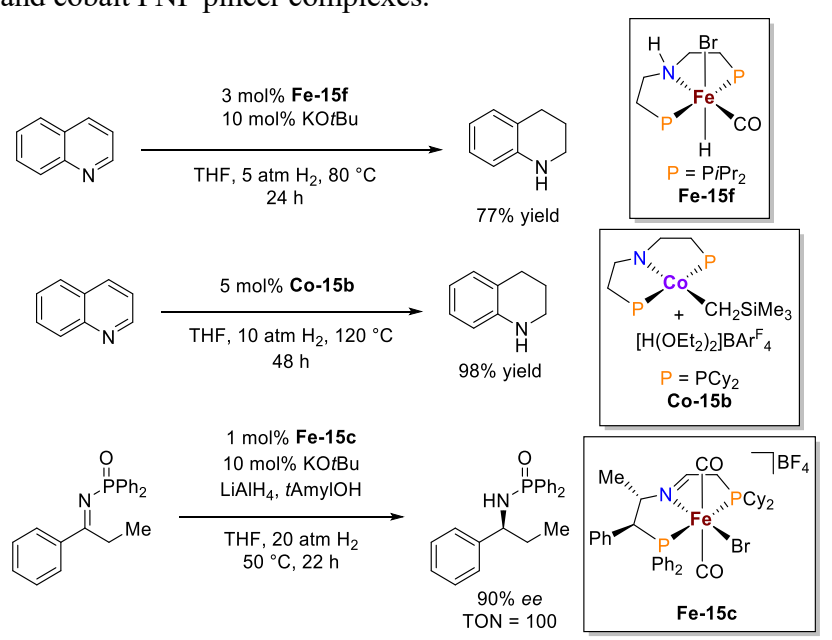


Cobalt catalyzed imine hydrogenation (Scheme 40) has been reported with Hanson's catalyst Co- $\mathbf{1 5 b}$. ${ }^{195}$ Jones and coworkers further expanded the substrate scope for a range of $N$-heterocycles in THF at $120{ }^{\circ} \mathrm{C}(5 \mathrm{~mol} \%$ catalyst loading, 10 atm $\left.\mathrm{H}_{2}\right) .{ }^{447}$

\subsection{Hydrogenation of Amides}

Amide hydrogenation to the respective amines and alcohols was first described with an iron pincer catalyst by Milstein and coworkers. ${ }^{448}$ The $\mathbf{P N}{ }^{\mathbf{P y}} \mathbf{P}$ iron complex Fe-1c catalyzes the hydrogenation of activated, electron deficient amides at high temperatures and pressure with comparably high catalyst loadings (Scheme 41). The strong base $\mathrm{KN}\left(\mathrm{SiMe}_{3}\right)_{2}$ (KHMDS), rather than more common $\mathrm{KO} t \mathrm{Bu}$, was used for activation of Fe-1c enabling the hydrogenation of twelve trifluoroacetamides. The borohydride complex Fe-1d exhibits slightly lower activity, yet does not require addition of base like Fe-1c. A mechanism via a hemiaminal intermediate was proposed, in analogy to ester hydrogenation, which is discussed in Section 3.5. ${ }^{449}$

Scheme 41. Amide hydrogenation with precatalyst Fe-1c. ${ }^{448}$

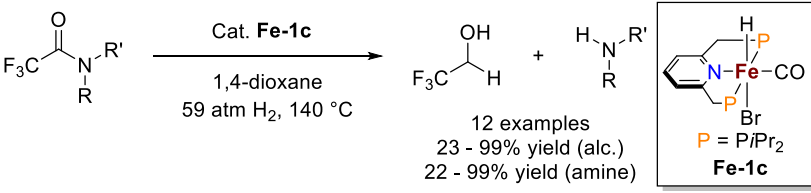

$$
\begin{aligned}
& \text { 至 } \\
& 2 \mathrm{~mol} \% \mathrm{Fe}-1 \mathrm{c} \quad 5 \mathrm{~mol} \% \mathrm{Fe}-1 \mathrm{c} \quad 5 \mathrm{~mol} \% \mathrm{Fe}-1 \mathrm{c} \quad 5 \mathrm{~mol} \% \mathrm{Fe}-1 \mathrm{c} \\
& 6 \mathrm{~mol} \% \text { KHMDS } 15 \mathrm{~mol} \% \text { KHMDS } 15 \mathrm{~mol} \% \text { KHMDS } 15 \mathrm{~mol} \% \text { KHMDS } \\
& \begin{array}{crrr}
12 \mathrm{~h} & 36 \mathrm{~h} & 36 \mathrm{~h} & 36 \mathrm{~h}
\end{array} \\
& \text { yield (alc.): } 99 \% \quad \text { yield (alc.): } 99 \% \quad \text { yield (alc.): } 36 \% \quad \text { yield (alc.): } 23 \%
\end{aligned}
$$

Langer and coworkers first reported iron pincer catalyzed hydrogenation of non-activated amides, ${ }^{185}$ using ${ }^{\mathrm{Et}} \mathbf{F e}-\mathbf{1 5 g}$ as catalyst. The sterically less demanding $\mathrm{PEt}_{2}$-ligand had been previously shown to be more efficient in ester hydrogenation than the popular ligands ${ }^{\mathrm{R}} \mathbf{P N}{ }^{\mathrm{H}} \mathbf{P}(\mathrm{R}=i \mathrm{Pr}, \mathrm{Cy}) .{ }^{450}$ Nine different amides and lactams were hydrogenated under basefree conditions at milder conditions and lower catalyst loadings than with pyridyl pincer complex Fe-1c. However, most lactams required harsh conditions and high catalyst loading.

In a consecutive study, Sanford and coworkers compared the activities of $\left({ }^{\mathrm{R}} \mathbf{P N}{ }^{\mathrm{H}} \mathbf{P}\right) \mathrm{Fe}\left(\mathrm{BH}_{4}\right)(\mathrm{H})(\mathrm{CO})(\mathrm{R}=\mathrm{Et}, \mathrm{Cy}, i \mathrm{Pr})$ in amide hydrogenation with additional $\mathrm{K}_{3} \mathrm{PO}_{4}$ as base for catalyst activation. ${ }^{451}$ In fact, ${ }^{\mathbf{C y}} \mathbf{F e}-\mathbf{1 5 g}$ exhibited the best performance while ${ }^{\mathbf{E t}} \mathbf{F e}-\mathbf{1 5 g}$ was by far inferior. Strong advantages of Sanford's protocol are the relatively low precatalyst loadings $(0.33 \mathrm{~mol} \%)$ and moderate $\mathrm{H}_{2}$ pressures $(20$ atm) needed for formamide hydrogenation. Interestingly, reproducibility of the catalytic rates required the use of research grade $\mathrm{H}_{2}(99.9999 \%)$.
Table 4. Hydrogenation of amides with iron precatalysts ${ }^{\mathbf{E t}} \mathbf{F e}-\mathbf{1 5 g}$ and ${ }^{\mathrm{Cy}} \mathbf{F e}$-15g. ${ }^{185,451}$

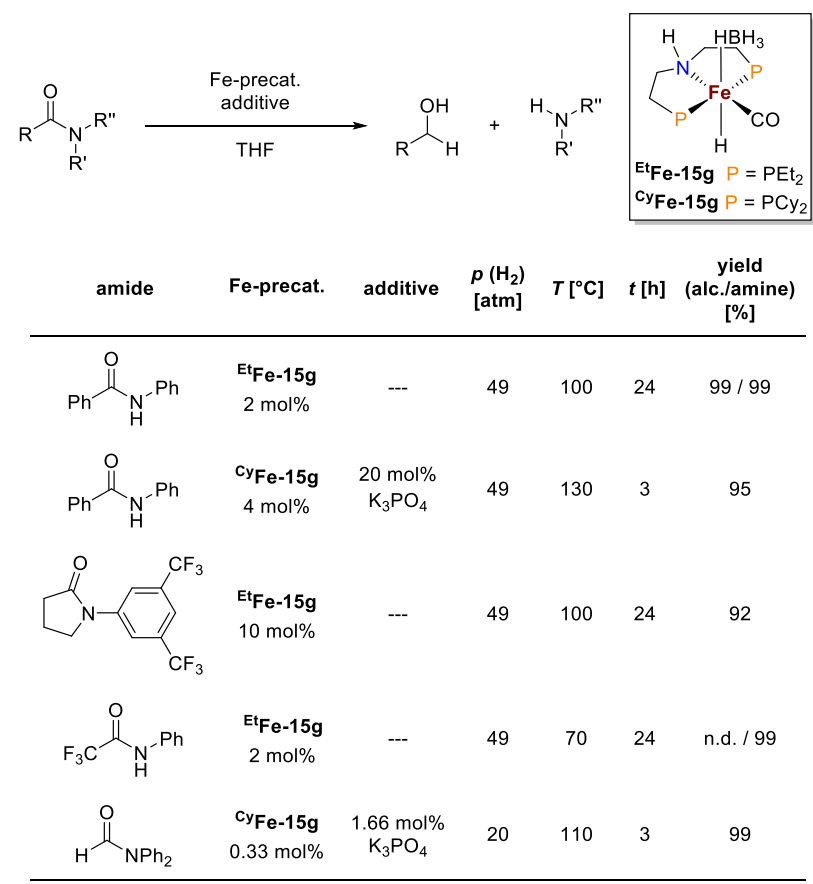

Jayarathne et al. employed the related amide complex ${ }^{i \mathbf{P r}} \mathbf{F e}-$ 15a for the hydrogenation of (form)amides with a very low catalyst loading of $0.07 \mathrm{~mol} \%$ (Scheme 42). ${ }^{452}$ Turnover numbers up to 5600 could be obtained. Interestingly, the authors found the addition of an excess $N$-phenylformamide significantly increased the yield and therefore the turnover number (Scheme 42). Subsequent mechanistic examinations attribute this effect for $\mathrm{N}, \mathrm{N}$-disubstituted amide substrates to metal-free, organocatalytic cleavage of the hemiaminal intermediate to formaldehyde and secondary amines. ${ }^{453} \mathrm{In}$ consequence, initial outer-sphere hydride transfer to the amide substrate is the relevant step for catalyst design. Furthermore the influence of Lewis-acid additives was examined. Increased yield was observed upon addition of lithium triflate. The combination of both promoters leads to a nearly additive enhancement effect.

Scheme 42. Amide hydrogenation precatalyst ${ }^{i \mathbf{P r}} \mathbf{F e}-\mathbf{1 5 a} .{ }^{452}$

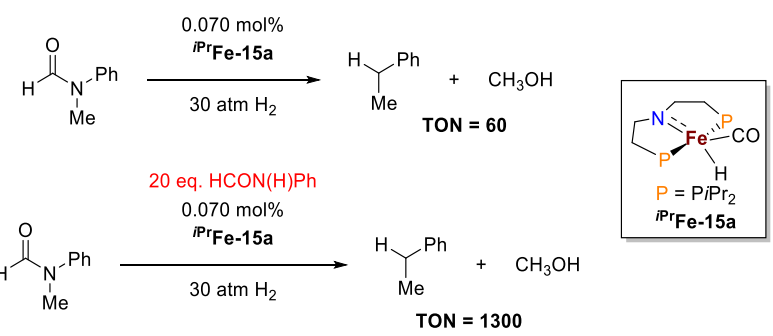

Beller and co-workers reported the catalytic upgrading of $\mathrm{CO}$ to ethylene glycol. ${ }^{454}$ The first step comprises palladiumcatalyzed, aerobic coupling of $\mathrm{CO}$ and piperidine to the oxamide $\left(\mathrm{C}(\mathrm{O}) \mathrm{NC}_{5} \mathrm{H}_{10}\right)_{2}$. Catalyst screening $(\mathrm{Ru}$ and $\mathrm{Fe})$ for subsequent hydrogenolysis of the amide intermediate gave high yields in ethylene glycol for ${ }^{\mathrm{Et}} \mathbf{F e - 1 5 g}$ yet at higher 
AMIDE HYDROGENATION

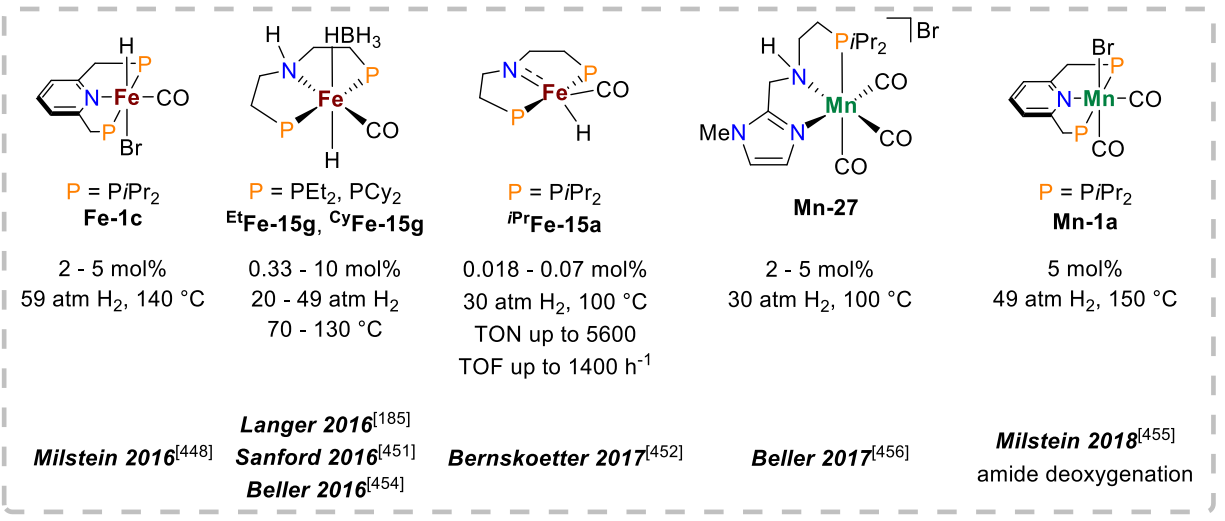

Figure 34. Summary of amide hydrogenation catalysts based on tridentate PNN and PNP ligands.

loadings of catalyst and base than for the related $\left({ }^{\mathbf{P h}} \mathbf{P N}{ }^{\mathrm{H}} \mathbf{P}\right) \mathrm{Ru}\left(\mathrm{BH}_{4}\right)(\mathrm{H})(\mathrm{CO})$.

Scheme 43. Deoxygenative hydrogenation of amides catalyzed by Mn-1a. ${ }^{455}$

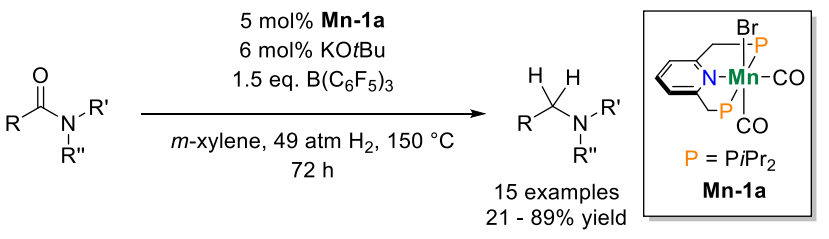

The pyridyl pincer manganese complex Mn-1a was employed in the deoxygenative hydrogenation of amides by Milstein and coworkers (Scheme 43). ${ }^{455}$ Stoichiometric amounts of Lewis acid additive $\mathrm{B}\left(\mathrm{C}_{6} \mathrm{~F}_{5}\right)_{3}$ increased the yield. Besides MLC $\mathrm{H}_{2}$ heterolysis, the dearomatized manganese complex also forms a dinuclear $\mathrm{N}_{2}$-complex.

Scheme 44. Amide hydrogenation with precatalyst Mn-27. ${ }^{456}$

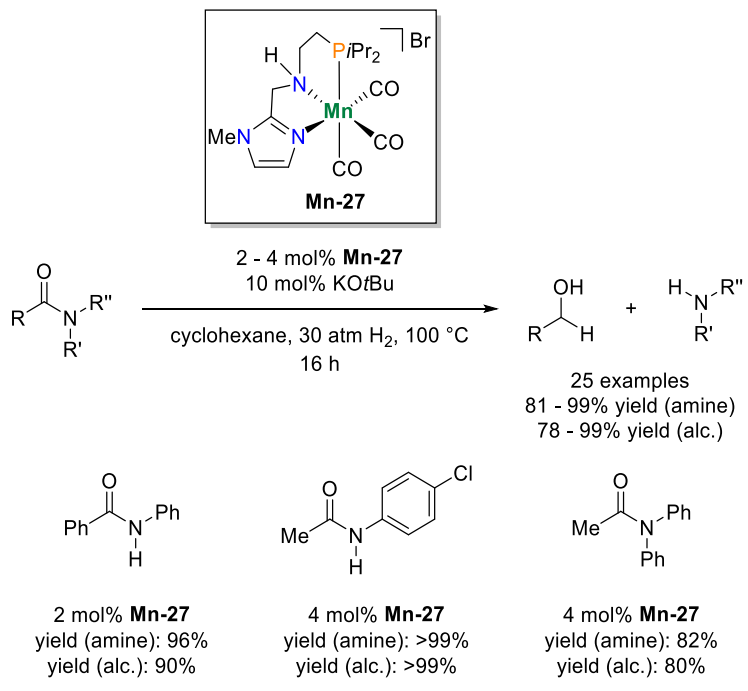

Beller and coworkers introduced the PNN pincer manganese complex Mn-27 into amide hydrogenation (Scheme 44). Isomerization to meridional chelation can be expected after CO dissociation. The catalytic protocol utilizes $2-4 \mathrm{~mol} \%$
Mn-27 and $10 \mathrm{~mol} \% \mathrm{KO} t \mathrm{Bu}$ at $100{ }^{\circ} \mathrm{C}$ in cyclohexane under $30 \mathrm{~atm} \mathrm{H}_{2}$. A broad substrate scope of 25 (form)amides were hydrogenated in moderate to quantitative yields.

Scheme 45. Hydrogenation of nitriles by precatalyst ${ }^{i P r} \mathbf{F e}-15 g$ (a: Isolated as well as GC yields). ${ }^{457}$
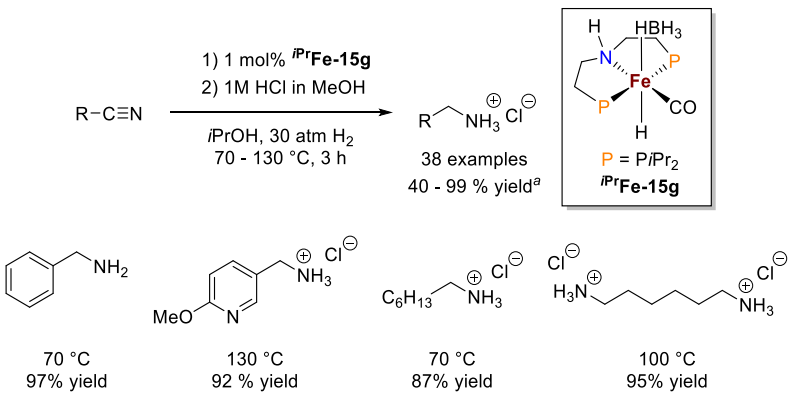

\subsection{Hydrogenation of Nitriles}

A comprehensive study on iron catalyzed nitrile hydrogenation was reported by Beller and coworkers in 2014 (Scheme 45). ${ }^{457}$ Borohydride complex ${ }^{i P r} \mathbf{F e}-15 g$ represented the first non-precious metal catalyst for nitrile hydrogenation to the respective ammonium salts after acidic workup. A vast range of (di)nitriles were converted in $i \mathrm{PrOH}$ as solvent without need for additional base giving moderate to almost quantitative amine yields. Excellent functional group tolerance was obtained for 24 aromatic nitriles and even 12 aliphatic nitriles which were reduced at relatively mild conditions and low catalyst loadings. Several aromatic and aliphatic dinitriles, like the polyamide production intermediate hexanedinitrile, were also converted in moderate to very good yields. Based on DFT computations, an all-iron(II) hydrideamine/amide outer-sphere mechanism was proposed with almost thermoneutral $\mathrm{H}_{2}$ addition to the iron amide state. ${ }^{457,458}$ Spectroscopic evidence for the iron(II) amide was presented, which could later be isolated by several groups. ${ }^{180,181}$ The relevance of MLC for catalysis is further supported by the lack of activity in case of the backbone $N$-methylated complex ${ }^{i P r}$ Fe-15Me. A comparative study on the catalyst series ${ }^{\mathrm{R}} \mathbf{F e - 1 5 g}(\mathrm{R}=\mathrm{Et}, \mathrm{Cy}, i \mathrm{Pr})$ revealed almost comparable nitrile hydrogenation activity for ${ }^{\mathrm{Cy}} \mathbf{F e}-\mathbf{1 5 g} .{ }^{459}$ 


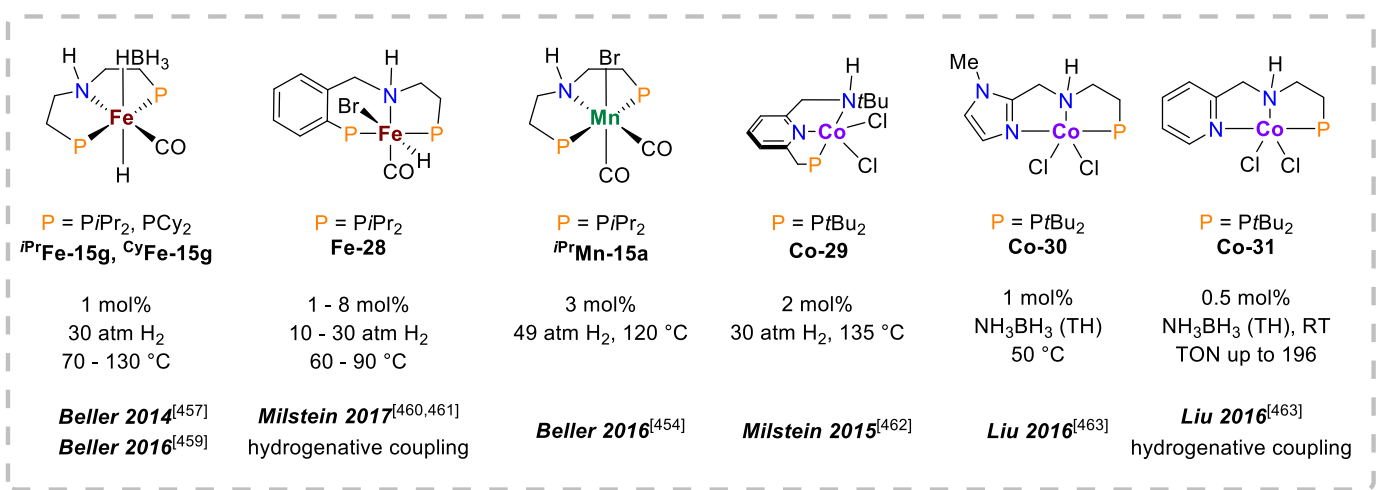

Figure 35. Summary of nitrile hydrogenation catalysts based on PNP and PNN pincer ligands.

Milstein and coworkers reported the reductive homocoupling of aromatic and aliphatic nitriles to secondary imines and ammonia, catalyzed by the unsymmetric pincer catalyst Fe-28/KO $t \mathrm{Bu}$ (Scheme 46). ${ }^{460}$ Aromatic nitriles generally give higher yields with comparably lower catalyst loadings. Cross coupling to unsymmetrically substituted aldimines $\mathrm{RHC}=\mathrm{NR}$ ' can be achieved with the same catalyst starting from nitriles $\mathrm{RCN}$ and amines $\mathrm{H}_{2} \mathrm{NR}$ ', respectively, at considerably milder reaction conditions ( $1 \mathrm{~mol} \% \mathrm{Fe}-28 / \mathrm{KO} t \mathrm{Bu}$, $\left.10-20 \mathrm{~atm} \mathrm{H}_{2}, 60{ }^{\circ} \mathrm{C}\right) .{ }^{461} \mathrm{~A}$ range of 20 different aldimines was synthesized with a similar substrate scope compared to reductive nitrile homocoupling.

Scheme 46. Reductive coupling of nitriles to secondary imines catalyzed by Fe-28. ${ }^{460}$ (a: Yields determined by GC-MS and ${ }^{1} \mathrm{H}$ NMR spectroscopy).

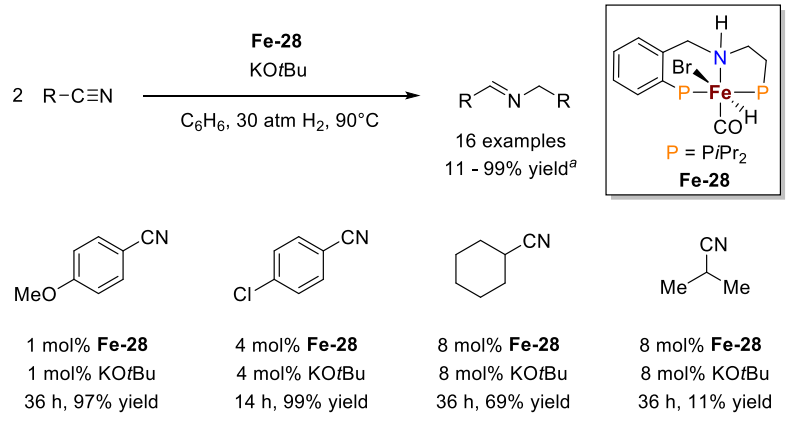

The authors proposed a pathway via the condensation of primary imine and amine intermediates (Scheme 47). Spectroscopic examination revealed the formation of an iron(II) monohydride amide complex from reaction of $\mathbf{F e - 2 8}$ with base that adds $\mathrm{H}_{2}$. In turn the resulting amine dihydride transfered $\mathrm{H}_{2}$ to benzonitrile in a stoichiometric control experiment, reforming the amide species. These results support a MLC mechanism. Interestingly, a cis-dihydride configuration was found for the $\mathrm{H}_{2}$-addition product which is the minor isomer in case of $\left({ }^{\mathbf{i P r}} \mathbf{P} \mathbf{N}^{\mathbf{H}} \mathbf{P}\right) \mathrm{Fe}(\mathrm{H})_{2} \mathrm{CO} .{ }^{181}$ Evidently, secondary imine formation requires the accumulation of a primary imine intermediate. It is therefore tempting to correlate the different nitrile hydrogenation selectivities of the structurally strongly related catalysts to hydricities of cis- and trans-dihydride isomers.
Scheme 47. Proposed mechanism for iron PNP pincer catalyzed hydrogenation of nitriles to secondary imines $\left(\mathrm{P}={\left.\mathrm{P} i \mathrm{Pr}_{2}\right)}^{460}\right.$

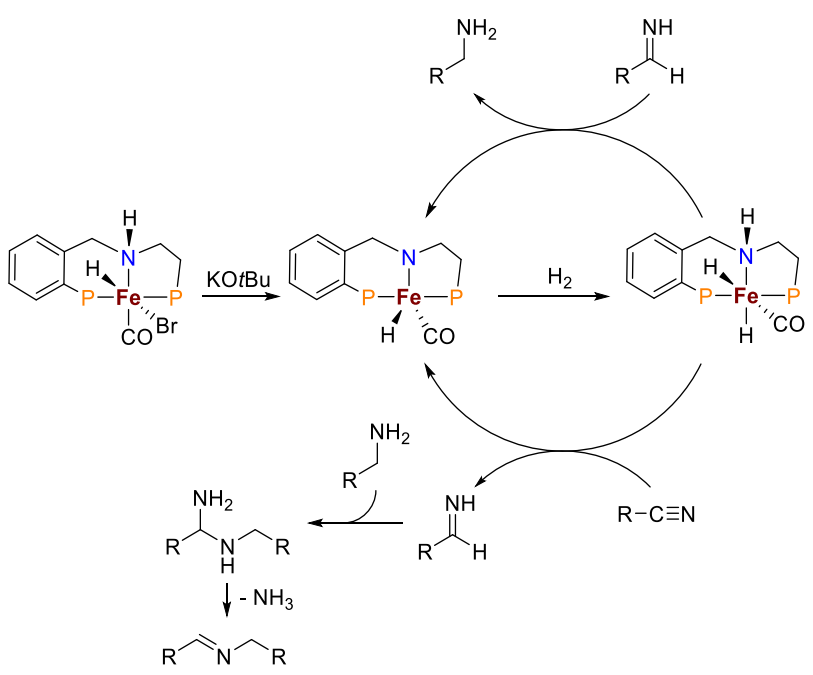

The cobalt catalyst Co-29 was utilized by Milstein and coworkers for the hydrogenation of 23 (hetero)aromatic, benzylic and aliphatic nitriles to primary amines (Scheme 48) at comparatively forcing conditions $\left(135{ }^{\circ} \mathrm{C}, 30 \mathrm{~atm} \mathrm{H}_{2}\right) .{ }^{462}$ Catalyst ( $2 \mathrm{~mol}^{\%}$ ) activation required $2 \mathrm{~mol}_{\%} \mathrm{NaBEt}_{3} \mathrm{H}$ and $4.4 \mathrm{~mol} \% \mathrm{NaOEt}$, respectively, as additives. Benzylic and aliphatic amines generally give lower yields. For pentanenitrile and heptanenitrile as substrates, secondary amines were observed, which is rationalized with reductive nitrile coupling as for the iron catalyst Fe-28, ${ }^{460,461}$ and subsequent hydrogenation of the secondary imine intermediate. The authors propose a monoanionic, dearomatized phosphinediamide cobalt(I) hydride complex as active catalyst species.

Scheme 48. Hydrogenation of nitriles catalyzed by precatalyst $\mathrm{Co}-$ 29. ${ }^{462}$

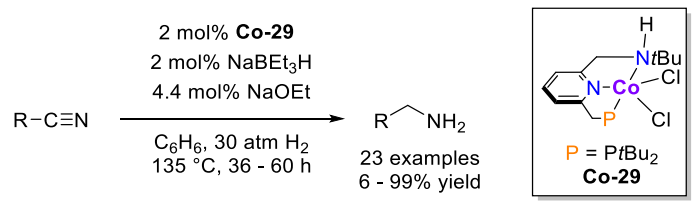

Versatile, base-free transfer hydrogenation of nitriles with ammonia borane as the hydrogen source was described by 


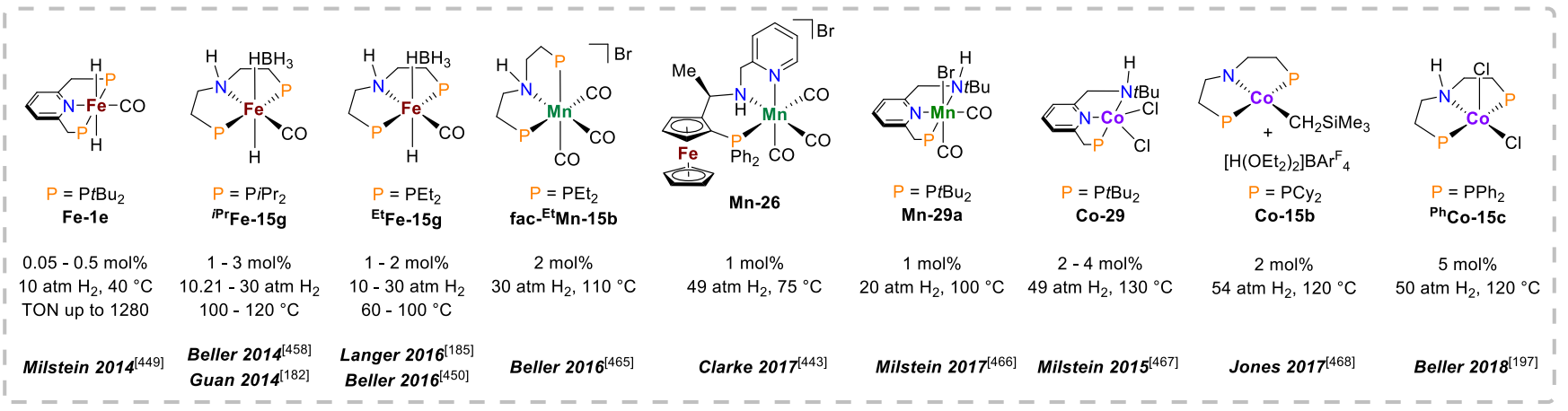

Figure 36. Summary of Ester Hydrogenation Catalysts Based on Tridentate PNP and PNN Ligands.

the groups of Zhou and Liu utilizing related cobalt(II) PNN pincer precatalysts with divergent selectivities (Scheme 49). ${ }^{463}$ Transfer hydrogenation (TH) of nitriles to the respective primary amines is catalyzed by Co-30 $(1 \mathrm{~mol} \%)$ in $n$ hexane at $50{ }^{\circ} \mathrm{C}$. The substrate scope covered 26 (hetero)aromatic and alipathic (di)nitriles. TH of aliphatic (di)nitriles requires elevated temperatures. In contrast, hydrogenative homocoupling to secondary amines is observed with precatalyst $\mathbf{C o - 3 1}$ in $\left(\mathrm{CF}_{3}\right)_{2} \mathrm{CHOH}$ as solvent. A range of $20 \mathrm{ni}-$ triles could be coupled via this methodology in moderate to good yields with high tolerance of reducible groups. Tertiary amines can be obtained from reductive cross-coupling of nitriles with a secondary amine. Pincer $N$-methylated analogs of the precatalysts did not significantly affect the yields suggesting an inner-sphere mechanism without MLC.

Scheme 49. Nitrile hydrogenation and hydrogenative coupling with precatalysts $\mathbf{C o - 3 0}$ and $\mathbf{C o - 3 1}{ }^{463}$

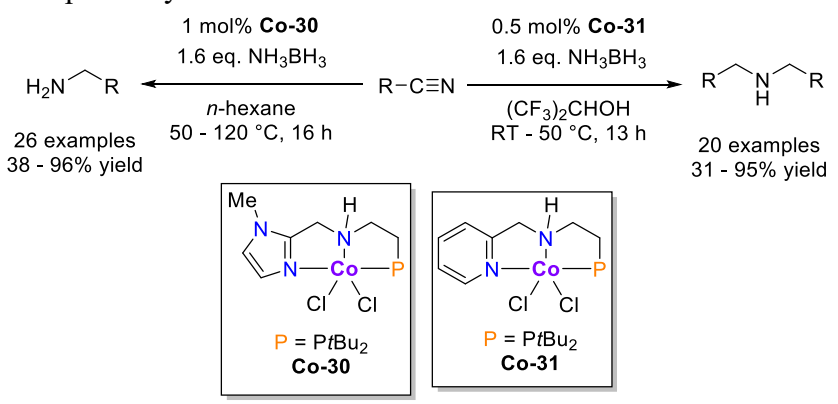

\subsection{Hydrogenation of Esters}

The iron pincer catalyzed hydrogenation of esters was first reported by Milstein and coworkers (Scheme 50). ${ }^{449}$ The pyridyl-pincer dihydride complex Fe-1e catalyzes the hydrogenation of activated trifluoroacetic esters in the presence of base $(\mathrm{KO} t \mathrm{Bu})$. A TON up to 1280 could be obtained for the hydrogenation of 2,2,2-trifluoroethyl trifluoroacetate. The authors suggested a mechanism that is related to their initial proposal for ketone hydrogenation via carboxylate insertion into a dihydride $\mathrm{M}-\mathrm{H}$ bond and $\mathrm{MLC} \mathrm{H}_{2}$ heterolysis. Dissociation of the hemiacetal intermediate and subsequent trifluoroacetaldehyde hydrogenation were proposed as pathway to the final products.
Scheme 50. Hydrogenation of trifluoracetic esters, bicarbonate and $\mathrm{CO}_{2}$ with catalyst $\mathbf{F e}-1 \mathbf{e}^{241,449}$

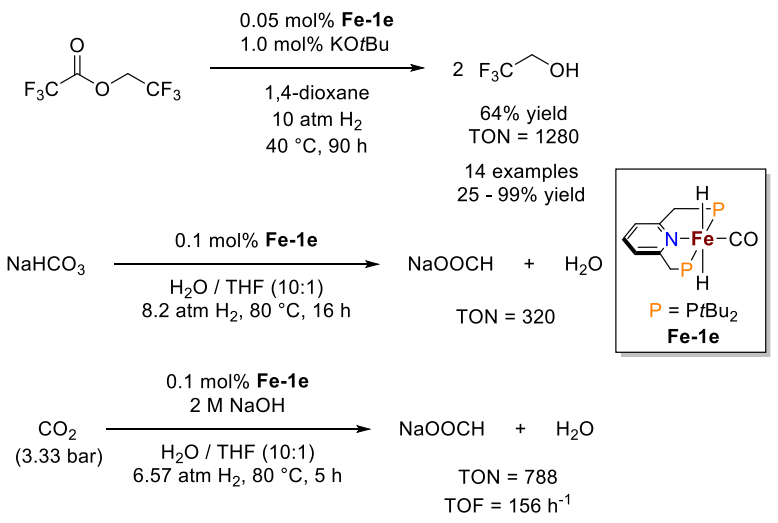

Shortly after, the groups of Beller and Guan independently reported the first examples for hydrogenation of nonactivated esters, ${ }^{182,458}$ while Hazari and Schneider described reverse acceptorless alcohol dehydrogenation to esters as discussed in Section 4.1.1. ${ }^{424}$ All groups used iron ${ }^{\text {iPr }} \mathbf{P N}{ }^{\mathrm{H}} \mathbf{P}-$ based catalysts. Beller and coworkers employed the borohydride complex ${ }^{i \operatorname{Pr}} \mathbf{F e}$-15g for ester hydrogenation under basefree conditions with high functional group tolerance at high $\mathrm{H}_{2}$ pressure $(30 \mathrm{~atm})$ and temperature $\left(100-120{ }^{\circ} \mathrm{C}\right)$ with $1 \mathrm{~mol} \%$ catalyst loading (Scheme 51). ${ }^{458}$ Selective ester reduction was obtained for methyl-4-decenoate as substrate. In comparison, Guan's protocol requires higher loadings in ${ }^{\text {PrF }} \mathbf{F e}-15 \mathrm{~g}(3 \mathrm{~mol} \%)$ yet at lower pressure (10 atm) and was also applied to a broad substrate scope, ${ }^{182}$ and subsequently to an industrially derived sample (CE-1295) on a kilogram scale. ${ }^{464}$ Starting from the backbone $N$-methylated complex ${ }^{i P r} \mathbf{F e}-15 M e$ no conversion of methyl benzoate was obtained suggesting the relevance of MLC. ${ }^{458}$ More detailed experimental and computational examinations were reported for reverse acceptorless alcohol dehydrogenation with this catalyst platform, which is discussed in Section 4.1.1. ${ }^{424} \mathrm{Bel}-$ ler and Langer independently reported improved catalytic activity for the sterically less encumbered ${ }^{\mathrm{Et}} \mathbf{F e}-\mathbf{1 5 g}$ (Scheme 51) allowing for reduction of the temperature $60-70{ }^{\circ} \mathrm{C}$ and pressure $(10 \mathrm{~atm})$ for reference substrate methylbenzoate. ${ }^{185,450}$ 
Scheme 51. Hydrogenation of methylesters and lactones with $\left(\mathbf{P N}^{\mathbf{H} / \mathbf{M e}} \mathbf{P}\right)$ iron precatalysts. ${ }^{185,450,458}$
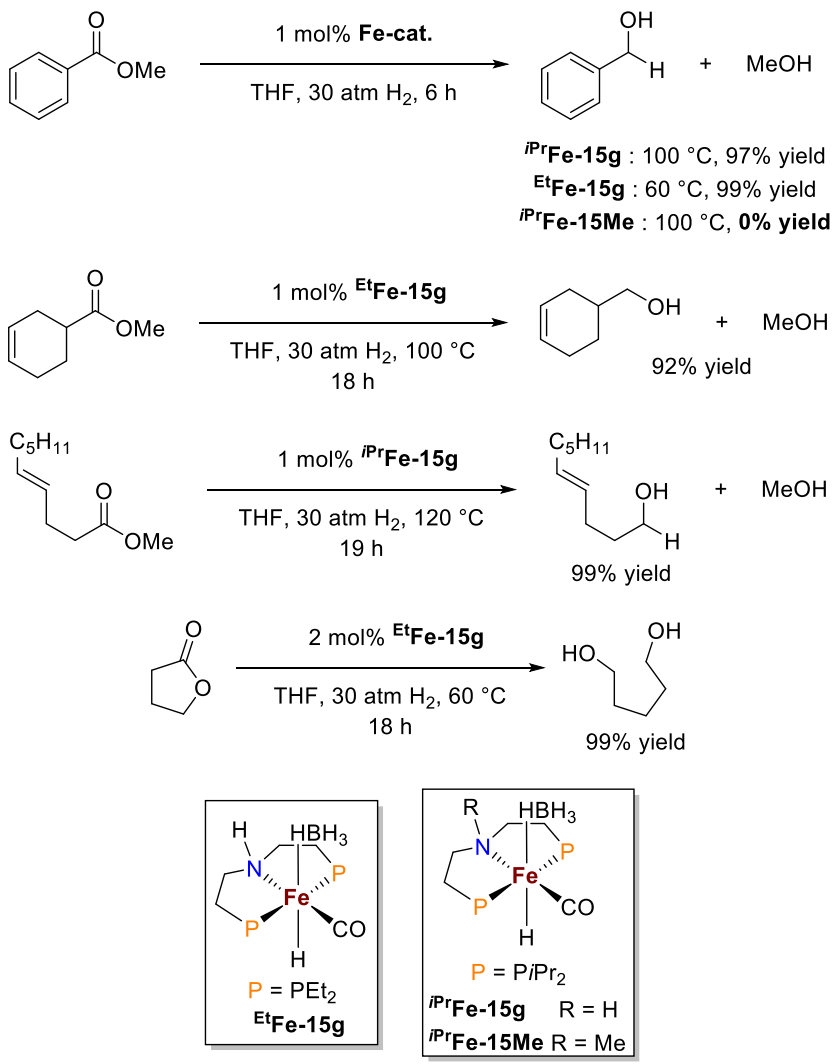

Expansion to manganese catalyzed ester hydrogenation was first reported by the Beller group. ${ }^{465}$ The manganese(I) precatalysts ${ }^{i \mathbf{P r}} \mathbf{M n - 1 5 a}$ and fac- ${ }^{\mathrm{Et}} \mathbf{M n - 1 5 b}$ (Scheme 52) exhibit comparable catalytic performance towards methyl benzoate. The authors propose formation of the same amido dicarbonyl complex, $\left({ }^{\mathrm{Et} P N P}\right) \mathrm{Mn}(\mathrm{CO})_{2}$, upon activation with base as the catalytically active species. Isomerization of fac${ }^{\mathrm{Et}} \mathrm{Mn}-15 \mathrm{~b}$ after deprotonation and $\mathrm{CO}$ dissociation to mer( $\left.{ }^{\text {EtPNP}}\right) \mathrm{Mn}(\mathrm{CO})_{2}$ was computed to be exergonic by $7.7 \mathrm{kcal} \cdot \mathrm{mol}^{-1}$. A broad range of 27 esters was reduced with fac- ${ }^{\text {Et } M n-15 b}$, including (hetero)aromatic as well as aliphatic esters and lactones. Remote $\mathrm{C}=\mathrm{C}$ double bonds were retained under the catalytic conditions, while $\alpha, \beta$-unsaturated esters were reduced to the saturated alcohols. Stepwise ester to alcohol reduction via a hemiacetal intermediate was proposed based on DFT computations. The highest barrier was obtained for the first outer-sphere hydride transfer reaction suggesting catalyst optimization based on $\mathrm{Mn}-\mathrm{H}$ hydricities.
Scheme 52. Hydrogenation of esters using precatalysts fac- ${ }^{\text {Et }} \mathbf{M n -}$ 15b and ${ }^{\text {Et }} \mathbf{M n - 1 5 a}$, respectively (a: Isolated yields as well as yields determined by GC; b: identical result for ${ }^{\mathbf{E t}} \mathbf{M n - 1 5 a}$; c: 48 h; d: 3phenylpropanol as product). ${ }^{465}$

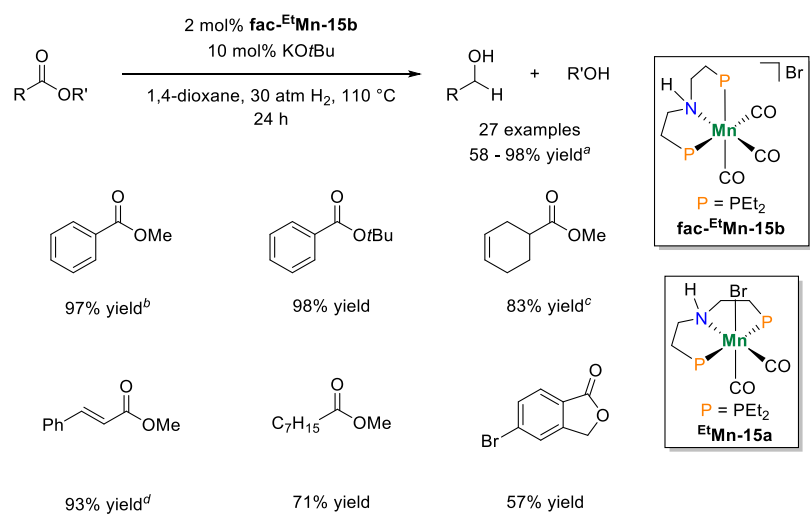

The ketone hydrogenation precatalyst Mn-26 (Scheme 38, Section 3.1.3.) is also active in the hydrogenation of esters with similar activities as Beller's manganese catalyst. ${ }^{443} \mathrm{~A}$ range of eight (di)esters were hydrogenated to the respective alcohols in moderate to quantitative yields at $75^{\circ} \mathrm{C}$ over $18 \mathrm{~h}$ in $i \mathrm{PrOH}$. Only the para-nitro derivative showed no conversion and aliphatic esters were hydrogenated with lower yields.

Scheme 53. A) Hydrogenation of esters and lactones with precatalyst Mn-29a (a: Yields determined by GC). B) Stoichiometric control experiments. ${ }^{466}$
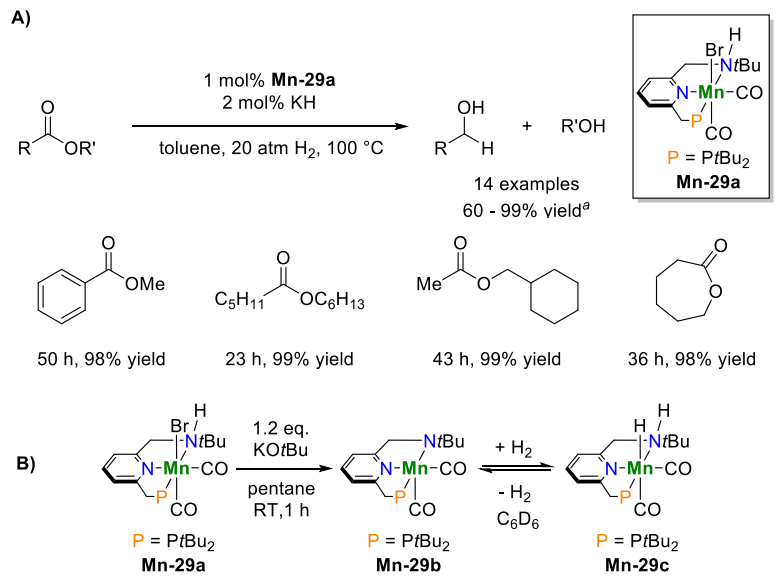

The pyridine based PNN manganese complex Mn-29a ( $1 \mathrm{~mol} \%)$ was used for reduction of 14 aromatic and aliphatic esters and lactones $\left(100{ }^{\circ} \mathrm{C}, 20 \mathrm{~atm} \mathrm{H}_{2}\right)$ with predominantly very good yields (Scheme 53 ). ${ }^{466}$ The catalyst is activated by the unusual base KH (2 mol\%). Stoichiometric control experiments show that deprotonation of the secondary amine ligand is favored over dearomatization. The structurally characterized amido pincer complex Mn-29b reversibly splits dihydrogen by MLC to the amine monohydride complex Mn-29c. Based on these observations, the authors propose a catalytic cycle via outer-sphere hydrogenation and MLC $\mathrm{H}_{2}$ heterolysis, emphasizing the relation to the aliphatic ${ }^{\mathrm{R}} \mathbf{P} \mathbf{N}^{\mathrm{H}} \mathbf{P}$. 
Milstein and co-workers introduced the cobalt PNN pincer complex Co-29 for ester hydrogenation (Scheme 54). ${ }^{467}$ The electronic structure of Co-29 was not reported but high-spin cobalt(II) can be expected based on M-L bond lengths. Upon activation with $\mathrm{KO} t \mathrm{Bu}$ and sodium superhydride a range of 11 different esters could be reduced in moderate to good yields. Interestingly, the authors observed an unusual selectivity. The generally more challenging aliphatic ester substrates were converted in contrast to aromatic and activated trifluoroacetic acid esters. As an explanation, the authors propose a mechanism via initial ester enolization with the strong base $\mathrm{KO} t \mathrm{Bu}$ and subsequent cobalt catalyzed $\mathrm{C}=\mathrm{C}$ hydrogenation of the enolate. In consequence, benzoates and trifluoroacetic acid esters are not enolizable. This mechanism implies preferential $\mathrm{C}=\mathrm{C}$ over $\mathrm{C}=\mathrm{O}$ reduction by a cobalt hydride, as reflected by the milder conditions of alkene over ketone hydrogenation observed for Hanson's cobalt aminopincer system (Scheme 31). ${ }^{195}$

Scheme 54. Hydrogenation of esters with precatalyst Co-29. ${ }^{467}$

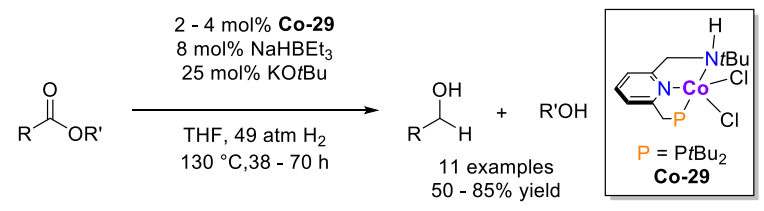

Hanson's catalyst, $\mathbf{C o - 1 5 a} /\left[\mathrm{H}\left(\mathrm{Et}_{2} \mathrm{O}\right)_{2}\right] \mathrm{BAr}_{4}{ }_{4}(\mathbf{C o}-\mathbf{1 5 b})$, was also employed in ester hydrogenation by Jones and co-workers. ${ }^{468}$ Without further additives 11 substrates could be hydrogenated to alcohols, including aliphatic and aromatic esters with $2 \mathrm{~mol} \%$ cobalt loading at $120{ }^{\circ} \mathrm{C}$ and $54 \mathrm{~atm} \mathrm{H}_{2}$ (Scheme 55). Interestingly, a pincer $N$-methylated analogous complex exhibited only slightly reduced catalytic activity. The authors therefore proposed an inner-sphere mechanism without MLC. However, as pointed out above there remains some ambiguity regarding the nature of Hanson's catalyst, which is discussed in Section 3.1.2.

Scheme 55. Hydrogenation of esters with $\mathrm{Co}\left({ }^{\mathrm{R}} \mathbf{P N}{ }^{\mathrm{H}} \mathbf{P}\right)$ catalysts. ${ }^{197,468}$

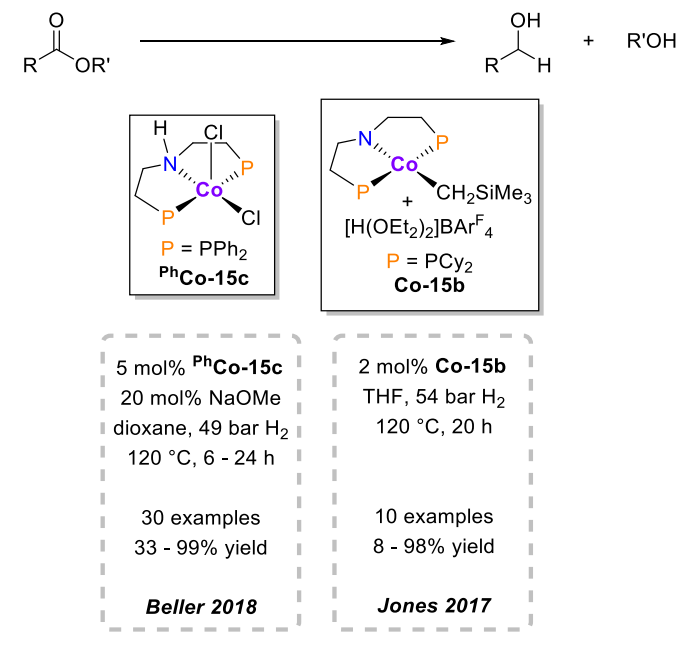

The hydrogenation of a broad range of 30 different esters was reported by Beller and coworkers starting from ${ }^{\text {Ph }} \mathbf{C o -}$
$15 \mathrm{c}(5 \mathrm{~mol} \%)$ and $\mathrm{NaOMe}(20 \mathrm{~mol} \%)$ with predominantly good yields (Scheme 55). ${ }^{197}$ The substrate scope includes many carbo- and heterocyclic, aliphatic and aromatic (di)esters as well as lactones. The authors note, that a black precipitate is observed at $140{ }^{\circ} \mathrm{C}$ after 24 hours attributed to cobalt nanoparticles. However, mercury and $\mathrm{PMe}_{3}$ poisoning experiments were unsuccessful and the $\mathrm{N}$-methylated pincer complex exhibited no activity in the hydrogenation of methyl benzoate and methyl octanoate.

\subsection{Hydrogenation of $\mathrm{CO}_{2}$}

Several products can arise from $\mathrm{CO}_{2}$ hydrogenation that range in principle from formic acid and $\mathrm{CO}$ (2-electron reduction), formaldehyde $\left(4 \mathrm{e}^{-}\right)$and methanol $\left(6 \mathrm{e}^{-}\right)$all the way to methane $\left(8 \mathrm{e}^{-}\right)$or other hydrocarbons. ${ }^{469}$ Regarding the $2-$ electron products, formic acid or $\mathrm{CO}$, both reactions are endergonic at ambient conditions in the gas phase. However, formic acid formation can be driven by product deprotonation in polar solvents. Besides this thermochemical argument, the reaction of transition metal hydrides with $\mathrm{CO}_{2}$ usually leads to formates, which is therefore called the normal insertion, thereby determining formate selective catalysis. ${ }^{206}$ $\mathrm{CO}_{2}$ hydrogenation in basic aqueous conditions is further complicated by substrate solvation and the nature of the true hydrogenated species, $\mathrm{CO}_{2}$ or (bi)carbonate, is not always clear.

Scheme 56. Proposed mechanism for $\mathrm{CO}_{2}$ hydrogenation with catalyst Fe-1e $\left(\mathrm{P}=\mathrm{P} t \mathrm{Bu}_{2}\right) .{ }^{241}$

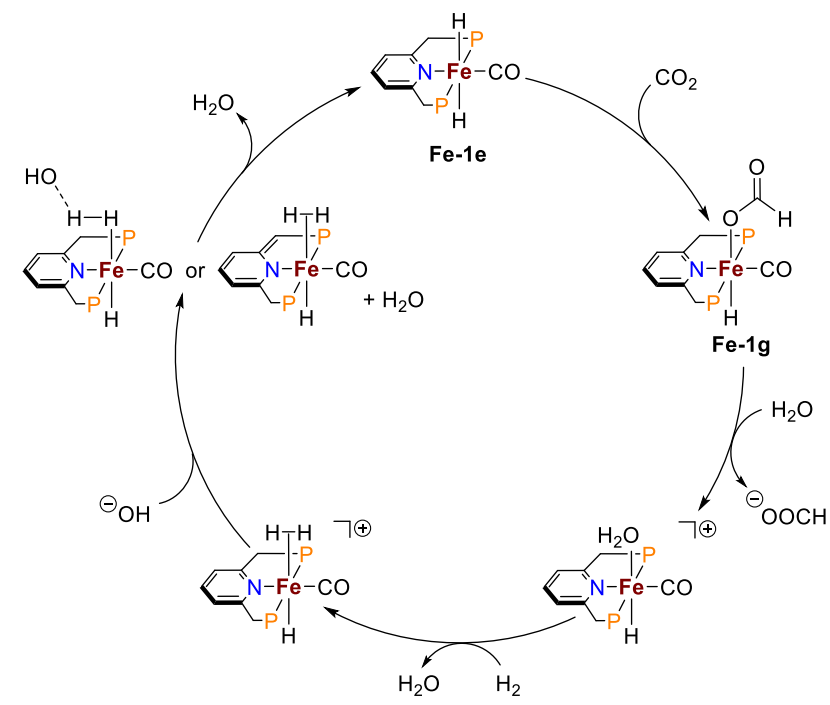

3.6.1. Pyridyl-Based Pincer Catalysts First-row metal catalysts have made a strong impact in $\mathrm{CO}_{2}$ hydrogenation and electroreduction and the role of molecular iron catalysts with functional ligands has recently been comprehensively reviewed ${ }^{470}$ For hydrogenation to formic acid, the low formate hydricity $\left(44 \mathrm{kcal} \cdot \mathrm{mol}^{-1}\right.$ in $\left.\mathrm{MeCN}\right)$ requires a catalyst with strong tendency for hydride transfer. ${ }^{68}$ The trans-dihydride configuration which is stabilized by the pincer framework for iron complexes and weakens the $\mathrm{Fe}-\mathrm{H}$ bonds should therefore be an ideal platform. Accordingly, Langer et al. reported in 2011 the hydrogenation of sodium 
$\mathrm{CO}_{2}$ HYDROGENATION

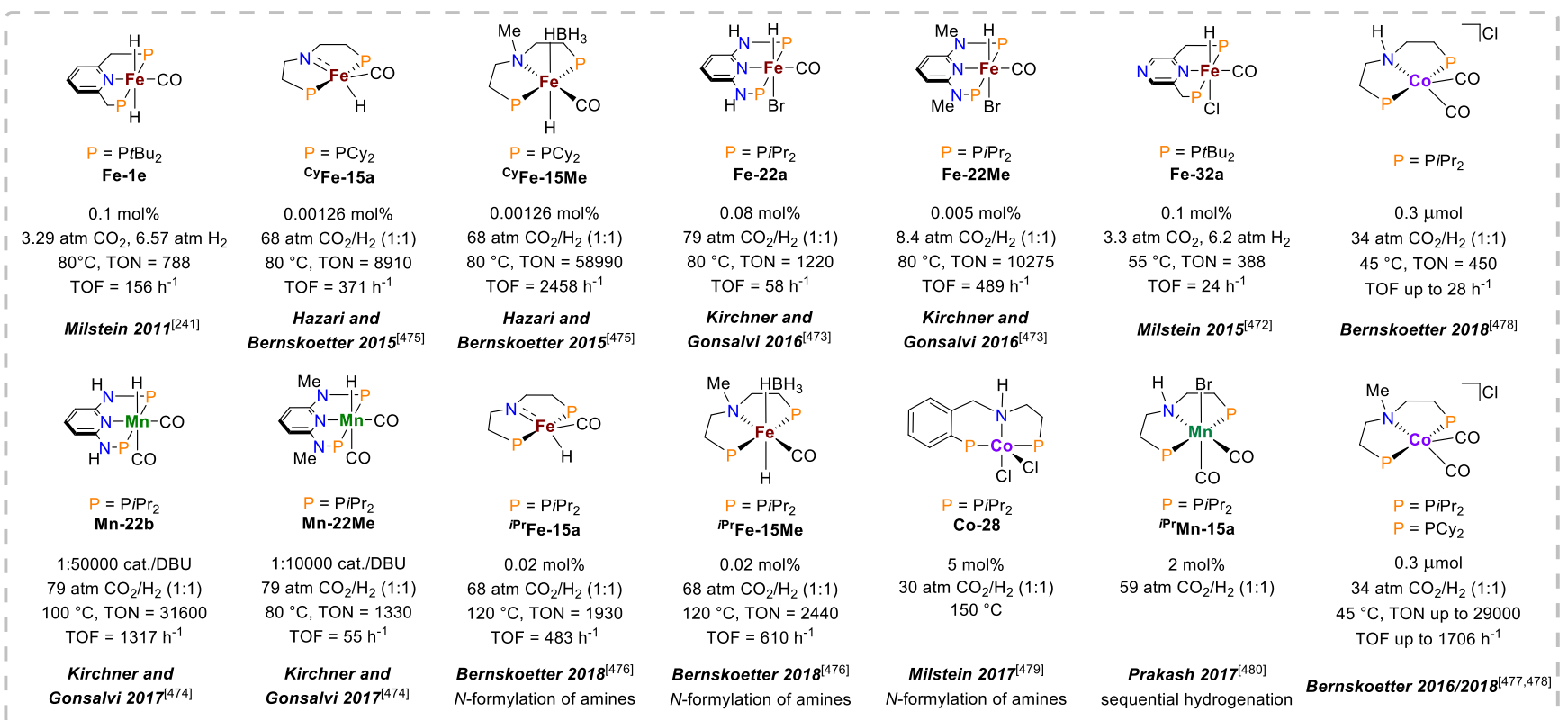

Figure 37. Summary of $\mathrm{CO}_{2}$ hydrogenation catalysts based on PNP pincer ligands.

bicarbonate and $\mathrm{CO}_{2}$ to formate using the pincer complex $\left({ }^{t \text { Bu}} \mathbf{P N} N^{P y} \mathbf{P}\right) \mathrm{Fe}(\mathrm{H})_{2} \mathrm{CO}(\mathbf{F e}-1 \mathbf{e})$ as catalyst (Scheme 50). ${ }^{241}$ Relatively mild reaction conditions $\left(80{ }^{\circ} \mathrm{C}, 8-10\right.$ atm $\mathrm{H}_{2} / \mathrm{CO}_{2}$ ) were leading to turnover numbers and frequencies (up to $156 \mathrm{~h}^{-1}$ ) that could for the first time compete with molecular noble metal catalysts. ${ }^{471}$

Scheme 57. Hydrogenation of sodium bicarbonate and $\mathrm{CO}_{2}$ catalyzed by $\mathbf{F e - 3 2 a}$ and proposed mechanism $\left(\mathrm{P}=\mathrm{P} t \mathrm{Bu}_{2}\right){ }^{472}$
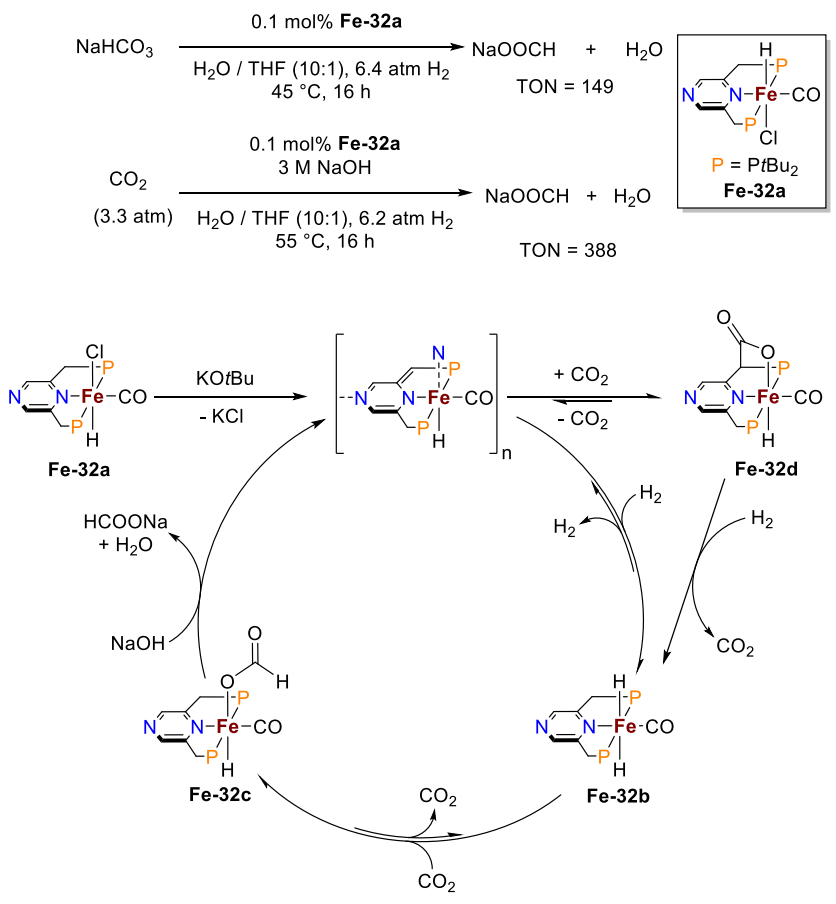

A mechanism was proposed based on stoichiometric control experiments (Scheme 56) with $\mathrm{CO}_{2}$ inserting into the metal hydride bond in the catalytically active dihydride species Fe1e. After hydrolysis of the formate intermediate Fe-1g, $\mathrm{H}_{2}$ - heterolysis closes the catalytic cycle. The role of MLC via ligand de-/aromatization for hydrogen activation remained unresolved.

The iron PNP pincer complex Fe-32a with an unusual pyrazine based backbone was also examined in $\mathrm{CO}_{2}$ hydrogenation (Scheme 57). ${ }^{472}$ Bicarbonate and $\mathrm{CO}_{2}$ hydrogenation exhibit lower TON compared with Fe-1e under otherwise identical conditions.

Table 5. Sodium bicarbonate and $\mathrm{CO}_{2}$ hydrogenation with iron $\mathbf{P N}_{3} \mathbf{P}$ Pincer catalysts Fe-22a and Fe-22Me. ${ }^{473}$

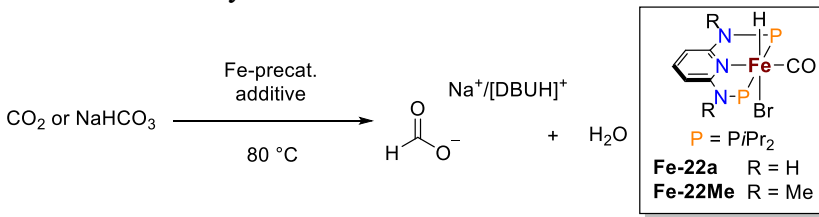

\begin{tabular}{ccccccc}
\hline $\begin{array}{c}\text { precat. } \\
(\text { mol\% })\end{array}$ & $\boldsymbol{p}$ [atm] & $\boldsymbol{t}[\mathrm{h}]$ & additive & solvent & yield [\%] & TON \\
\hline $\begin{array}{c}\mathrm{Fe}-22 \mathrm{a} \\
(0.08 \mathrm{~mol} \%)\end{array}$ & $\begin{array}{c}79 \\
\left(\mathrm{CO}_{2} / \mathrm{H}_{2} 1: 1\right)\end{array}$ & 21 & $\begin{array}{c}\mathrm{NaOH} \\
(10 \text { eq. })\end{array}$ & $\mathrm{H}_{2} \mathrm{O} / \mathrm{THF}$ & 98 & 1220 \\
$\begin{array}{c}\mathrm{Fe}-22 \mathrm{Me} \\
(0.005 \mathrm{~mol} \%)\end{array}$ & $\begin{array}{c}8.4 \\
\left(\mathrm{CO}_{2} / \mathrm{H}_{2} 1: 1\right)\end{array}$ & 21 & $\begin{array}{c}\mathrm{DBU} \\
(6.25 \mathrm{eq} .)\end{array}$ & $\mathrm{EtOH}$ & 21 & 10275 \\
$\begin{array}{c}\mathrm{Fe}-22 \mathrm{a} \\
(0.05 \mathrm{~mol} \%)\end{array}$ & $\begin{array}{c}89 \\
\left(\mathrm{H}_{2} / \mathrm{NaHCO}_{3}\right)\end{array}$ & 24 & --- & $\mathrm{H}_{2} \mathrm{O} / \mathrm{THF}$ & 98 & 1964 \\
$\begin{array}{c}\mathrm{Fe}-22 \mathrm{a} \\
(0.005 \mathrm{~mol} \%)\end{array}$ & $\begin{array}{c}89 \\
\left(\mathrm{H}_{2} / \mathrm{NaHCO}_{3}\right)\end{array}$ & 24 & --- & $\mathrm{H}_{2} \mathrm{O} / \mathrm{THF}$ & 23 & 4560 \\
$\begin{array}{c}\mathrm{Fe}-22 \mathrm{Me} \\
(0.05 \mathrm{~mol} \%)\end{array}$ & $\begin{array}{c}89 \\
\left(\mathrm{H}_{2} / \mathrm{NaHCO}_{3}\right)\end{array}$ & 24 & --- & $\mathrm{H}_{2} \mathrm{O} / \mathrm{THF}$ & 52 & 1034 \\
\hline & & & & & & \\
\hline
\end{tabular}

Stoichiometric control reactions gave rise to a proposed mechanism (Scheme 57). Interestingly, the deprotonation of precatalyst Fe-32a leads to polymerization of the dearomatized product via the bridging pyrazine. Deaggregation of the coordination polymer was considered to proceed either by direct $\mathrm{H}_{2}$ heterolysis or reversible $\mathrm{CO}_{2}$ 1,3-addition followed 
by hydrogenolysis. From the trans-dihydride product, an analogous mechanism was proposed as for parent Fe-1e.

Table 6. $\mathrm{CO}_{2}$ hydrogenation with manganese catalysts $\mathbf{M n - 2 2 b}$ and Mn-22Me. $^{474}$

\begin{tabular}{|c|c|c|c|c|c|c|}
\hline $\begin{array}{r}\mathrm{CO}_{2}+\mathrm{H}_{2} \\
(79 \mathrm{~atm} 1: 1)\end{array}$ & \multicolumn{2}{|c|}{$\begin{array}{c}\text { catalyst } \\
\text { DBU, additives }\end{array}$} & [DBU & ] $[\mathrm{HCOO}]$ & \multicolumn{2}{|c|}{ 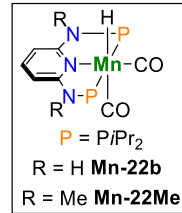 } \\
\hline catalyst & $\begin{array}{c}\text { cat./DBU } \\
\text { ratio }\end{array}$ & solvent & $T\left[{ }^{\circ} \mathrm{C}\right]$ & additive & yield [\%] & TON \\
\hline$M n-22 b$ & $1: 50000$ & $\mathrm{THF} / \mathrm{H}_{2} \mathrm{O}$ & 80 & - & 16 & 9100 \\
\hline $\mathrm{Mn}-22 \mathrm{Me}$ & $1: 10000$ & $\mathrm{EtOH}$ & 80 & - & 13 & 1330 \\
\hline $\mathrm{Mn}-22 \mathrm{Me}$ & $1: 50000$ & $\mathrm{THF} / \mathrm{H}_{2} \mathrm{O}$ & 100 & $\begin{array}{c}1 \mathrm{mmol} \\
\text { LiOTf }\end{array}$ & 63 & 31600 \\
\hline
\end{tabular}

Kirchner, Gonsalvi and coworkers used the $\mathbf{P N}_{\mathbf{3}} \mathbf{P}$ pincer ligated iron complex Fe-22a for sodium bicarbonate hydrogenation with high turnover number (up to 4560) and low catalyst loading $(0.005 \mathrm{~mol} \%)$ at high hydrogen pressure $(90$ bar) and $80{ }^{\circ} \mathrm{C}$ (Table 5). ${ }^{473}$ The backbone $N$-methylated catalyst Fe-22Me leads to lower yield compared with parent Fe-22a. $\mathrm{CO}_{2}$ hydrogenation in $\mathrm{H}_{2} \mathrm{O}$ /THF with precatalyst Fe-22a $(0.08$ mol\%) gave a turnover number of 1220 . Under optimized conditions, with the backbone $N$-methylated catalyst Fe-22Me high TON up to 10275 could be obtained. Computational examinations for this catalyst support a dihydride/formate cycle.

Table 7. Comparison of iron diphosphinoamine pincer catalysts in the $\mathrm{CO}_{2}$ hydrogenation. ${ }^{475}$

\begin{tabular}{|c|c|c|c|c|c|}
\hline $\begin{array}{l}\text { precat. } \\
\text { (mol\%) }\end{array}$ & $p$ [atm] & additive & yield [\%] & TON & $\begin{array}{l}P=P C y_{2} \\
C^{c} \mathrm{Fe}-15 a\end{array}$ \\
\hline $\begin{array}{c}{ }^{{ }^{y}} \mathrm{Fe}-15 a \\
(0.00126 \mathrm{~mol} \%)\end{array}$ & $\begin{array}{c}68 \\
\left(\mathrm{CO}_{2} / \mathrm{H}_{2} 1: 1\right)\end{array}$ & $\begin{array}{c}\text { DBU } \\
\text { (79600 eq.) } \\
\text { LiOTf } \\
\text { (10613 eq.) }\end{array}$ & 11 & 8910 & \\
\hline $\begin{array}{c}\mathrm{Cy}_{\mathrm{Fe}-15 \mathrm{Me}} \\
(0.00126 \mathrm{~mol} \%)\end{array}$ & $\begin{array}{c}68 \\
\left(\mathrm{CO}_{2} / \mathrm{H}_{2} 1: 1\right)\end{array}$ & $\begin{array}{c}\text { DBU } \\
\text { (79600 eq.) } \\
\text { LiOTf } \\
\text { (15920 eq.) }\end{array}$ & 74 & 58990 & $\begin{array}{c}\mathrm{H} \\
\mathrm{P}=\mathrm{PCy}_{2} \\
\mathrm{Cy}_{\mathrm{Fe}} \mathrm{Fe}-15 \mathrm{Me}\end{array}$ \\
\hline
\end{tabular}

Manganese(I) complexes with the same $\mathbf{P N}_{\mathbf{3}} \mathbf{P}$ ligands were also used for $\mathrm{CO}_{2}$ hydrogenation. Under optimized conditions, the monohydride dicarbonyl complex Mn-22b exhibits TON that are almost an order of magnitude higher than with Mn-22Me (Table 6). ${ }^{474}$ NMR experiments revealed the formation of a formate complex with $\mathrm{CO}_{2}$, which is also an active catalyst. Similar barriers for $\mathrm{H}_{2}$ activation pathways with and without MLC were obtained computationally. The yield could be further enhanced by addition of Lewis acids like LiOTf (Table 6). This effect was first reported and rationalized by Hazari and Schneider for the reverse formic acid decomposition and $\mathrm{CO}_{2}$ hydrogenation with iron pincer catalysts, which are discussed in Section 4.2 and 3.6.2, respectively. ${ }^{181,475}$
3.6.2. Diphosphinoamine Pincer Catalysts Particularly active catalysts could be obtained with diphosphineamino pincer catalysts. Hazari, Bernskoetter and co-

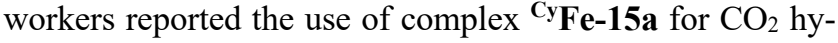
drogenation with high turnover numbers in the presence of DBU as a base and LiOTf (Table 7). ${ }^{475}$ The role of the Lewis acid co-catalyst was assumed to accelerated release of formate. The formate ligand that arises from $\mathrm{CO}_{2}$ insertion is in fact overstabilized by hydrogen bonding with the pincer secondary amine. Accordingly, the backbone $N$-methylated complex ${ }^{\mathrm{Cy}} \mathbf{F e}-\mathbf{1 5 M e}$ showed significantly increased activity with extraordinary turnover numbers (up to ca. 60000) emphasizing that the MLC pathway is not advantageous.

The CO-ligand of the catalyst was later exchanged with isonitriles, enabling steric and electronic tunability of the catalyst. ${ }^{192}$ However, the isonitrile ligated complexes exhibited turnover numbers that are about an order of magnitude lower compared with the $i s o$-propyl substituted amide com-

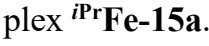

Scheme 58. $N$-Formylation of morpholine with $\mathrm{CO}_{2}$ as carbon source catalyzed by ${ }^{i P r}$ Fe-15a and ${ }^{i \operatorname{Pr}} \mathbf{F e}-15 M e .{ }^{476}$

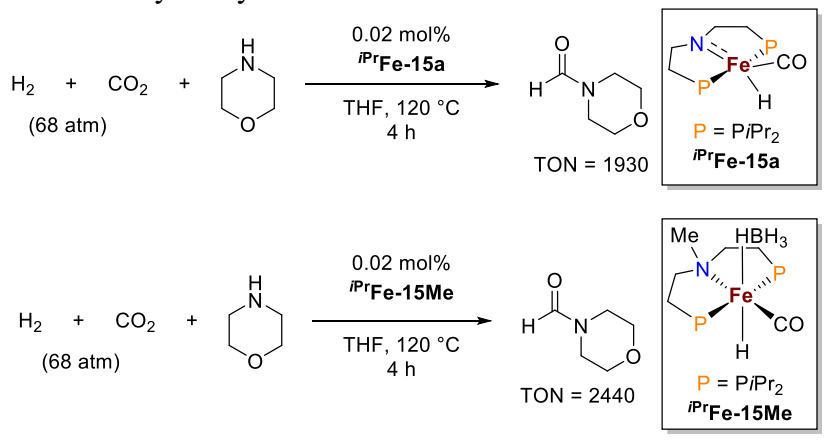

In the presence of amines, this catalyst can be used for $\mathrm{N}$ formylation with $\mathrm{CO}_{2}$ as $C_{1}$ source (Scheme 58). ${ }^{476}$ Selective morpholine $N$-formylation at high pressures and low catalyst loadings is observed. The backbone $N$-methylated borohydride complex ${ }^{i \mathbf{P r}} \mathbf{F e - 1 5 M e}$ gave the best turnover numbers up to 2440 .

The Bernskoetter group also reported the hydrogenation of $\mathrm{CO}_{2}$ with analogous aminopincer cobalt precatalysts. ${ }^{477,478}$ As in the iron case, the $N$-methylated pincer ligands gave higher activities than the secondary amine ligands. Furthermore, the authors propose a mechanism that includes pincer hemilability of the $N$-methylated derivative.

A related cobalt pincer catalyst was used by Milstein and coworkers for the selective amine $N$-formylation with $\mathrm{CO}_{2} / \mathrm{H}_{2}$ (Scheme 59). ${ }^{479}$ In the presence of a hydride source and base the cobalt(II) complex Co-28 catalyzed the $N$ formylation of 14 amines into the respective formamides in good to quantitative yields. The borohydride reagent reduces the precatalyst to a tetrahedral cobalt(I) complex, which exhibits catalytic activity comparable to Co-28. Furthermore, MLC-assisted $\mathrm{H}_{2}$ heterolysis by a T-shape cobalt(I) amido complex was proposed, followed by $\mathrm{CO}_{2}$ insertion, aminolysis and off-cycle formamide product formation (Scheme 
59). The electronic structure of the unusual cobalt(I) intermediates remains unresolved.

Scheme 59. $N$-Formylation of amines catalyzed by cobalt(II) precatalyst Co-28 (A) and proposed catalytic cycle (B). ${ }^{479}$

A)

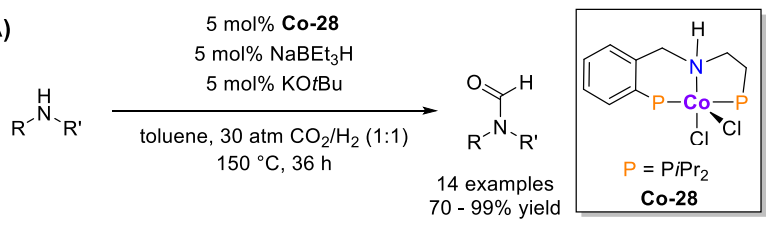

B)

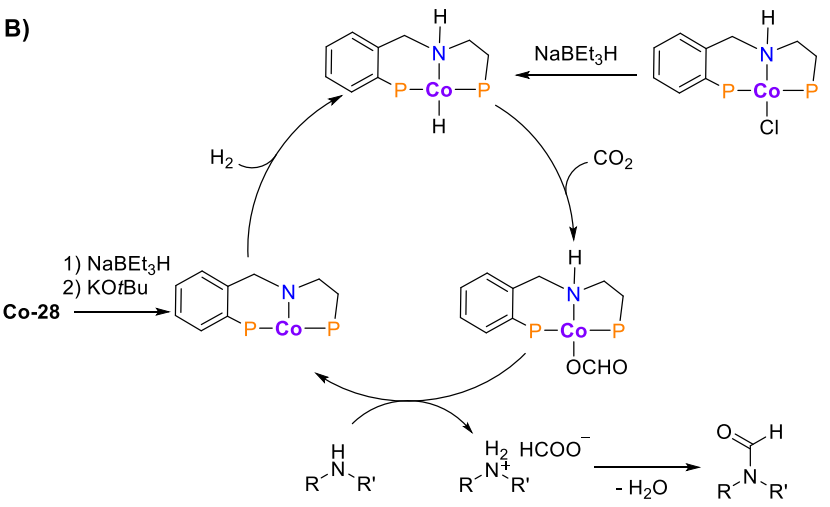

Scheme 60. Stepwise $\mathrm{CO}_{2}$ hydrogenation to methanol via formamide with precatalyst ${ }^{i P r} \mathbf{M n}-15 a(A)$ and proposed mechanism (B). ${ }^{480}$

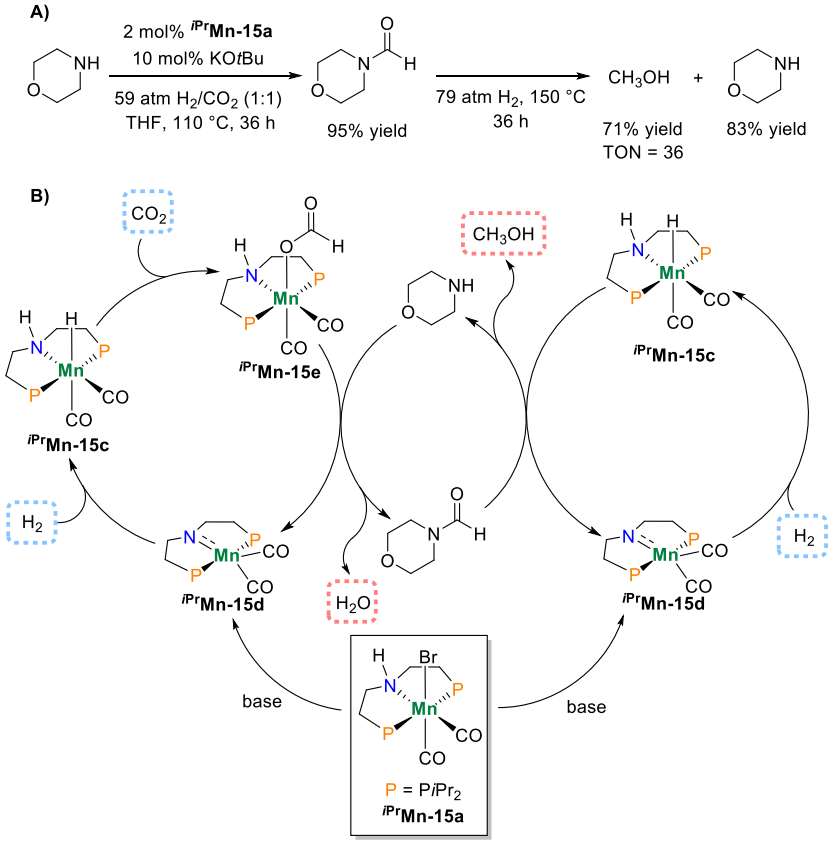

Prakash and coworkers reported an example for the sequential hydrogenation of $\mathrm{CO}_{2}$ all the way to the $6 \mathrm{e}^{-}$reduction product methanol catalyzed by the manganese(I) precatalyst ${ }^{i P r}$ Mn-15a (Scheme 60). ${ }^{480}$ The overall reaction is mediated by amine, which undergoes facile, catalytic $N$-formylation in the first step in the presence of ${ }^{i \mathbf{P r}} \mathbf{M n - 1 5 a}(2-4 \mathrm{~mol} \%)$ and base $(10 \mathrm{~mol} \%)$. The in situ formed formamides can then be further reduced at high $\mathrm{H}_{2}$ pressure (69-79 atm) to methanol in moderate to good yields. For the hydrogenation of morpholine-4-carbaldehyde a turnover number of 36 could be obtained. Based on NMR monitoring, the authors proposed a catalytic cycle with the manganese(I) hydride amine ( $\left.{ }^{\mathbf{P r}} \mathbf{M n - 1 5 c}\right)$ and amide species $\left({ }^{i \mathbf{P r}} \mathbf{M n - 1 5 d}\right)$ as the key intermediates for MLC-assisted $\mathrm{H}_{2}$ and $\mathrm{CO}_{2}$ activation (Scheme $60)$.

\subsection{Hydrogenation of Olefins}

3.7.1. Pyridyl-Based Pincer Catalysts. As one of the first examples in the renaissance of $3 \mathrm{~d}$ metal catalyzed hydroelementation, (PDI)Fe $\left(\mathrm{N}_{2}\right)_{2}(\mathbf{F e}-7 \mathbf{i})$ was shown to be an active catalyst in olefin hydrogenation (Scheme 61) with low catalyst loadings $(0.3 \mathrm{~mol} \%)$ at mild conditions (r.t., 1 atm $\left.\mathrm{H}_{2}\right) .{ }^{298,481}$ A wide range of substrates can be hydrogenated in high yield (>98\%). Terminal olefins are strongly favored over internal olefins and sterically demanding substrates. Chemoselective olefin hydrogenation is not affected by the presence of ester, tertiary amine, carbonyl, or ether substituents. However, reduced yield is obtained for primary amines, although this catalyst is active in azide hydrogenation to primary amines. ${ }^{297}$ Higher catalyst loadings (5 mol\%) are also required for some oxygen containing substrates and low conversion was obtained for a vinyl ester. ${ }^{158,298}$ Importantly, carbonyl groups were not hydrogenated.

Scheme 61. Olefin hydrogenation catalyzed by (PDI)Fe $\left(\mathrm{N}_{2}\right)_{2}(\mathbf{F e}-$ 7i) (Conversions determined by GC. or ${ }^{1} \mathrm{H}$ NMR spectroscopy). ${ }^{298,481}$

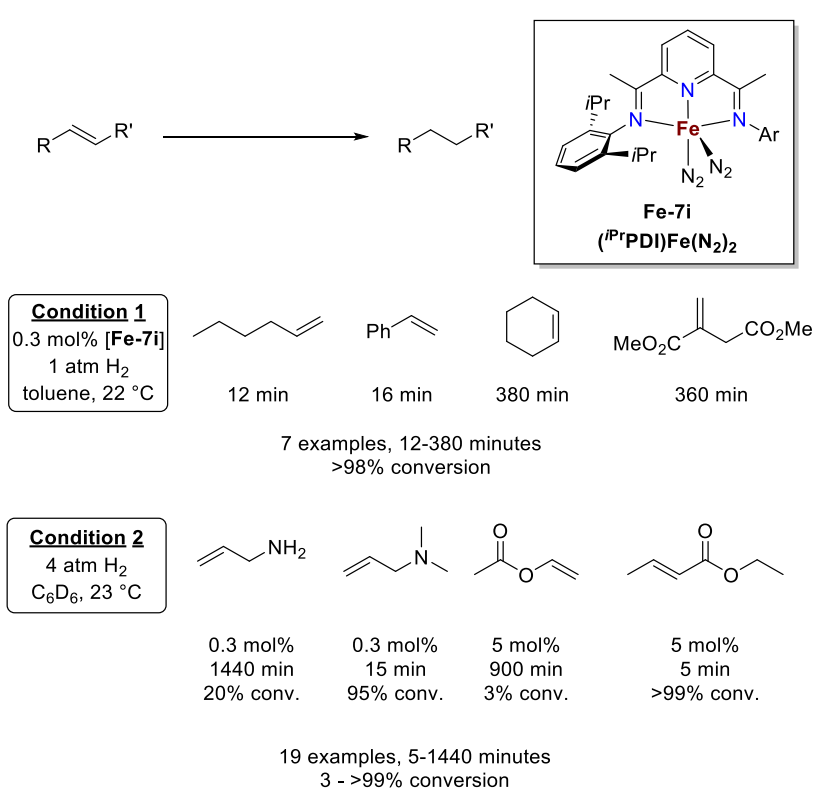

Within the proposed inner-sphere mechanism (Scheme 62) the catalytically active species is suggested to be formed by $\mathrm{N}_{2}$-dissociation, which opens a vacant coordination site for olefin coordination. Oxidative addition of dihydrogen furnishes a dihydride intermediate, which is followed by migratory insertion of the olefin into the metal-hydride bond. Reductive elimination of the alkane restores the catalytic active species. Importantly, both $\mathrm{H}_{2}$-oxidative addition and alkane reductive elimination steps are proposed to be redox neutral for the iron(II) center but rather ligand centered redox 


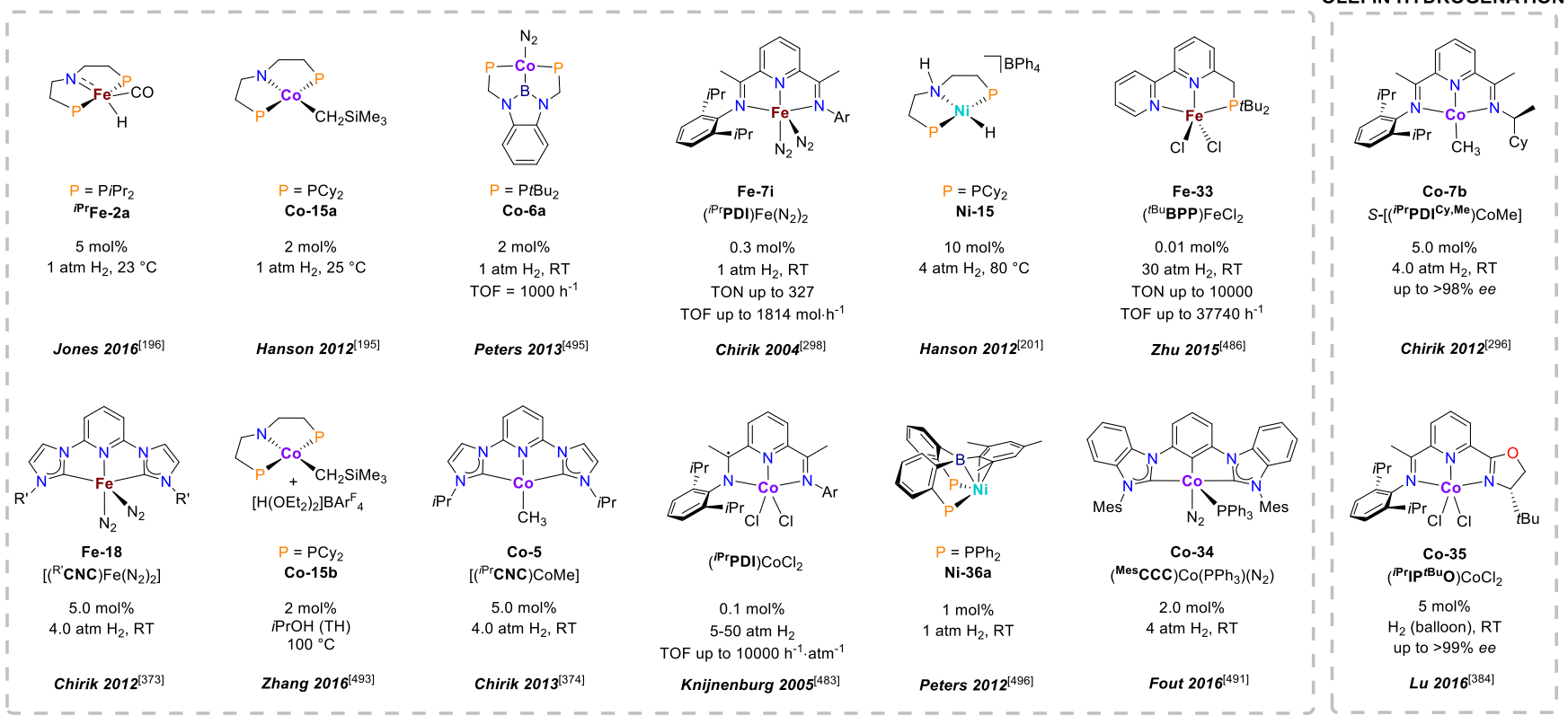

Figure 38. Summary of olefin hydrogenation catalysts based on tridentate PNP, PBP and PNN ligands.

processes, as shown for model reactions. ${ }^{146,148}$ In the absence of substrate, (PDI)Fe( $\left.\mathrm{N}_{2}\right)_{2}(\mathbf{F e}-7 \mathbf{i})$ forms dihydrogen and $\sigma$ $\mathrm{Si}-\mathrm{H}$ complexes under $\mathrm{H}_{2}$ atmosphere and with silanes, respectively. Oxidative addition might therefore be furnished by olefin coordination. ${ }^{298}$ In comparison, the saturated, redox innocent bis(phosphino)pyridine ligand ${ }^{i \mathbf{P r}} \mathbf{P N} \mathbf{N}^{\mathbf{P y}} \mathbf{P}$, which is a stronger donor, promotes metal centered oxidative addition. ${ }^{482}$ However, the resulting hydrides are much more thermally labile than their PDI counterparts, preventing catalytic applications to date.

Budzelaar and co-workers shortly afterwards reported the catalytic hydrogenation of olefins using (PDI)Co platforms as catalyst. Activation of high-spin $\left({ }^{i \mathbf{P r}} \mathbf{P D I}\right) \mathrm{CoCl}_{2}$ with excess $\mathrm{Al}(i \mathrm{Bu})_{3}$ enabled 1-octene hydrogenation at 5-49 atm $\mathrm{H}_{2}$ with high rates $\left(10000 \mathrm{~h}^{-1} \mathrm{~atm}^{-1}\right)$. Mono- and 1,2-disubstituted olefins are hydrogenated, while in case of 7-methyl1,6-octadiene only the terminal $\mathrm{C}=\mathrm{C}$ bond was reduced but not the trisubstituted olefin. NMR spectroscopic studies indicated that alkyl complexes $\left({ }^{i P r} \mathbf{P D I}\right) \mathrm{Co}(\mathrm{R})$ form the hydride ( ${ }^{\text {PrPDI}}$ PoH upon hydrogenolysis, which in turn inserts olefins into the $\mathrm{Co}-\mathrm{H}$ bond ${ }^{483,484}$ These observation lead to the proposed monohydride mechanism depicted in Scheme 62. However, an EPR signal assignable to a compound with ligand centered overall $\mathrm{S}=1 / 2$ spin-state was also observed in alkyl hydrogenolysis. The nature and relevance of this species for catalysis is not clear. ${ }^{483}$
Scheme 62: Proposed mechanism for (PDI)Fe- and (PDI) Co-catalyzed olefin hydrogenation. ${ }^{298,483}$
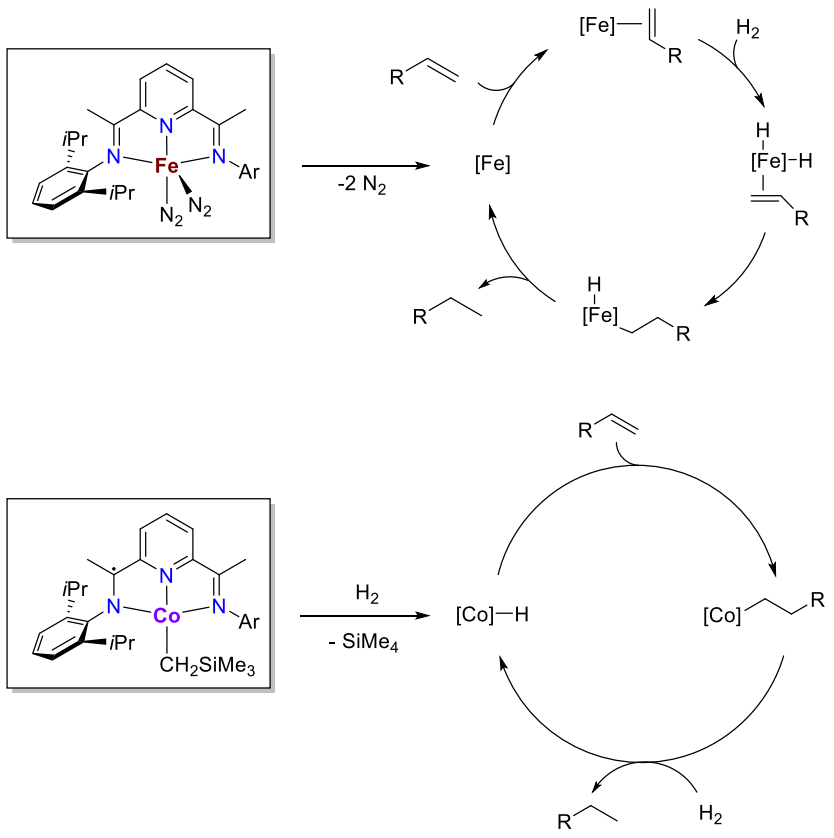

(PDI)Co catalyzed olefin hydrogenation was computationally evaluated by Budzelaar and more recently by Hopman, resulting in closely related mechanistic models. ${ }^{351,485}$ Both studies support a monohydride mechanism with migratory olefin insertion and hydrogenolysis by $\sigma$-bond metathesis (Scheme 63) over a dihydride pathway as proposed for iron. The (PDI)CoH hydride species is best described as low-spin cobalt(II) antiferromagnetically coupled to a $\mathbf{P D I}^{\mathbf{-}}$ radical anion. $^{484,485}$ (PDI)CoR alkyl intermediates exhibit closed shell cobalt(I) character or spin triplet multiplicity - from antiferromagenically coupled high-spin $\mathrm{Co}^{\mathrm{II}}$ and (PDI ${ }^{--}$) depending on the strength of the $\mathrm{C}-\mathrm{H}$ agostic interaction. ${ }^{485}$ Higher spin-states seem irrelevant for all other steps of the 
proposed catalytic cycle. It should be noted that the relative order of energetically closely spaced spin states can be highly dependent on the used functional due to varying degrees of electron exchange. The accessibility of closed-shell $\left(\mathbf{P D I}^{0}\right) \mathrm{Co}^{\mathrm{I}}$ and open-shell $\left(\mathbf{P D I}^{-}\right) \mathrm{Co}^{\mathrm{II}}$ states within the catalytic cycle augments the notion that ligand redox-activity might be a useful property for hydrogenation catalysis with this cobalt platform. However, Budzelaar's study proposed the same mechanism while only considering cobalt(I) closed-shell electronic configurations. ${ }^{483}$

Scheme 63. Computed mechanism for olefin hydrogenation with (PDI)Co as catalyst (AF-S: antiferromagnetically coupled singlet state; CS: closed-shell singlet state; AF-T: antiferromagnetically coupled triplet state). ${ }^{485}$

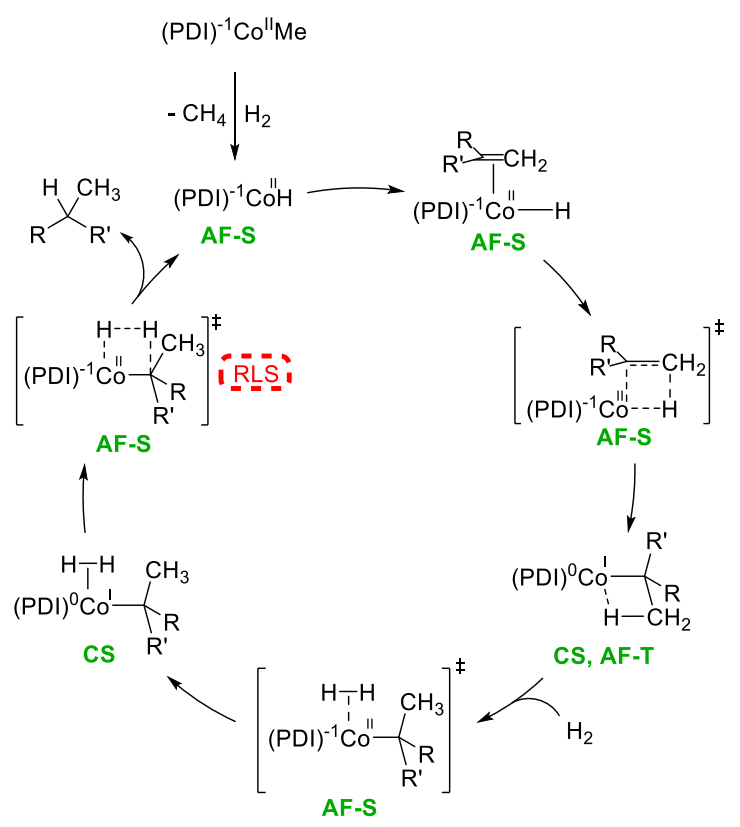

Zhu and co-workers introduced an iron PNN pincer catalyst Fe-33 with tremendously enhanced catalytic activity by tethering a strongly donating phosphine arm to a bipyridine core (Scheme 64). ${ }^{486}$ The phosphino bipyridine ligand enables hydrogenation of several terminal olefins with wide chemical group tolerance under $30 \mathrm{~atm}$ pressure at r.t. with low loadings $(0.1 \mathrm{~mol} \%)$ of the in situ reduced $\left(\mathrm{LiAlH}_{4}\right)$ iron precatalyst. The highest activity was found for styrene with a TOF of more than $37000 \mathrm{~h}^{-1}$. In contrast, the internal olefin cyclohexene is only partially converted, even at elevated temperatures.

In situ NMR-studies suggested an iron dihydride as active species, which gave rise to a proposed iron(0)/iron(II) mechanism via olefin migratory insertion and alkane reductive elimination. A potentially redox supporting role of the bipyridyl unit is not clear. Bipyridine ligands can adapt the mono radical form as well as closed shell dianionic form. ${ }^{142,387}$ Neidig, Milstein and coworkers showed in a crystallographic, spectroscopic, electrochemical and computational study that the $\mathrm{CO}$ complex $\left({ }^{\mathrm{R}} \mathrm{BPP}\right) \mathrm{Fe}(\mathrm{CO})_{2}$ is most accurately described as iron $(0)$ with strong backbonding into a bipyridine $\pi^{*}$-orbital. ${ }^{487}$
Scheme 64: Iron catalyzed olefin hydrogenation. Yield determined by GC. ${ }^{486}$

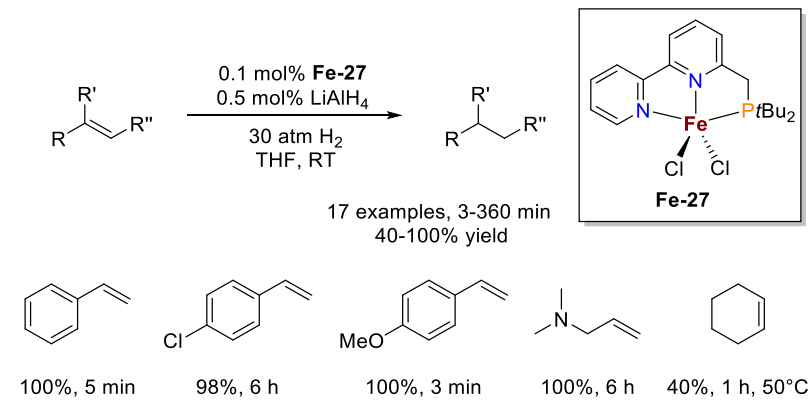

Chirik iron PDI catalysts were extensively utilized in C-Ccoupling, ${ }_{161,165,166,287-293}$ which was thoroughly reviewed. ${ }^{4,15,55,70,71,125,126,488}$ For example, catalyst $\mathbf{F e - 7 i}$ (Scheme 65) catalyzes the cycloaddition of $\alpha, \omega$-dienes. ${ }^{165}$ Subsequent expansion of the scope enabled the cyclization of 1,6-enynes and diynes coupled to product hydrogenation giving a range of saturated and unsaturated cyclopropanes. ${ }^{290}$ Catalyst loadings of $5 \mathrm{~mol} \%$ gave the products under mild conditions in generally high yields. The dienes obtained from reductive diyne cyclization are not further hydrogenated.

Scheme 65: Cyclization of 1,6 Enynes and Diynes followed by hydrogenation to substituted cyclopentanes. ${ }^{290}$ Yields referring to isolated substance.

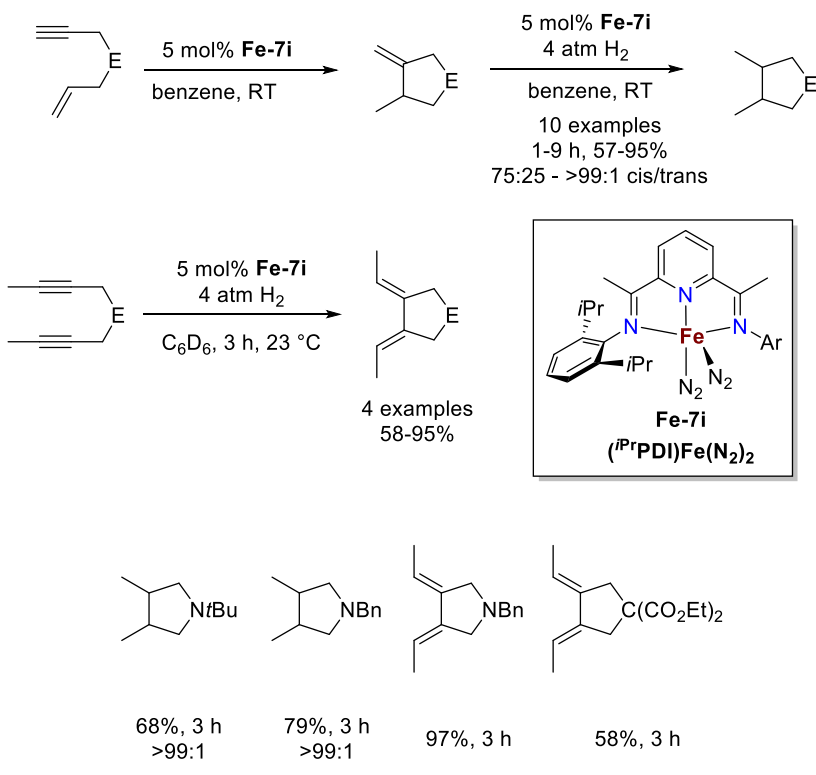

A mechanism was proposed based on deuterium labeling experiments (Scheme 66). The $\mathrm{Fe}^{\mathrm{II}}\left(\mathbf{P D I}^{2-}\right) / \mathrm{Fe}^{\mathrm{III}}\left(\mathbf{P D I}^{-}\right)$couple mediates the reductive two-electron coupling of the enyne substrate. Under $\mathrm{H}_{2}$, the metallacycle is opened by vinyl hydrogenolysis. $\mathrm{C}-\mathrm{H}$ reductive elimination restores the reduced ferrous iron state. Turn-over limiting hydrogenation is suggested by an H/D kinetic isotope effect of 6.0 under $\mathrm{D}_{2}$. 
Scheme 66: Proposed mechanism for iron-catalyzed reductive cyclization of 1,6-enynes. ${ }^{290}$
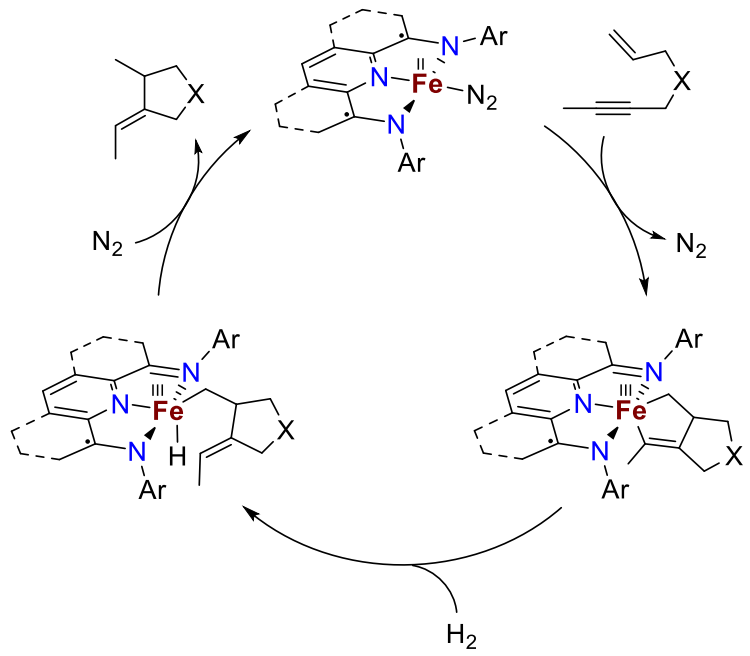

Ar = 2,6-diisopropylphenyl

Table 8. Comparison of $\mathrm{Fe}^{373}$ and $\mathrm{Co}^{374} \mathrm{PDI}$ and $\mathrm{CNC}$ type pincer catalysts in olefin hydrogenation (a: ( $\left.{ }^{\mathrm{Me}} \mathrm{PDI}\right) \mathrm{Fe}\left(\mathrm{N}_{2}\right)_{2}$ forms a dimer; b: conversion determined by GC-FID and by ${ }^{1} \mathrm{H}$ NMR; d: 50 $\left.{ }^{\circ} \mathrm{C}\right)$.

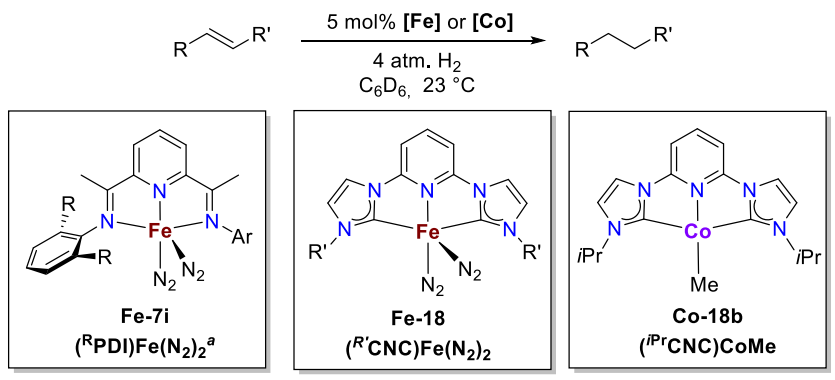

\begin{tabular}{|c|c|c|c|}
\hline subtrate & catalyst & time & conversion \\
\hline & $\left({ }^{(\mathrm{Pr} P D I}\right) \mathrm{Fe}\left(\mathrm{N}_{2}\right)_{2}$ & $24 \mathrm{~h}$ & $65 \%$ \\
\hline & $\left({ }^{\mathrm{Me}} \mathrm{PDI}\right) \mathrm{Fe}\left(\mathrm{N}_{2}\right)_{2}$ & $1 \mathrm{~h}$ & $>95 \%$ \\
\hline & $\left({ }^{(\mathrm{Pr}} \mathrm{CNC}\right) \mathrm{Fe}\left(\mathrm{N}_{2}\right)_{2}$ & $1 \mathrm{~h}$ & $>95 \%$ \\
\hline & $\left({ }^{\mathrm{Mes}} \mathrm{CNC}\right) \mathrm{Fe}\left(\mathrm{N}_{2}\right)_{2}$ & $1 \mathrm{~h}$ & $35 \%$ \\
\hline & $\left({ }^{(\mathrm{Pr}} \mathrm{CNC}\right) \mathrm{CoMe}$ & $1 \mathrm{~h}$ & $>95 \%$ \\
\hline & $\left({ }^{i \mathrm{Pr} P D I}\right) \mathrm{Fe}\left(\mathrm{N}_{2}\right)_{2}$ & $24 \mathrm{~h}$ & $32 \%$ \\
\hline & $\left({ }^{\mathrm{Me}} \mathrm{PDI}\right) \mathrm{Fe}\left(\mathrm{N}_{2}\right)_{2}$ & $24 \mathrm{~h}$ & $15 \%$ \\
\hline & $\left({ }^{(\mathrm{Pr}} \mathrm{CNC}\right) \mathrm{Fe}\left(\mathrm{N}_{2}\right)_{2}$ & $15 \mathrm{~h}$ & $>95 \%$ \\
\hline & $\left({ }^{\mathrm{Mes}} \mathrm{CNC}\right) \mathrm{Fe}\left(\mathrm{N}_{2}\right)_{2}$ & $1 \mathrm{~h}$ & $>95 \%$ \\
\hline & $\left({ }^{(P r} \mathrm{CNC}\right) \mathrm{CoMe}$ & $5 \mathrm{~h}$ & $>95 \%$ \\
\hline & $\left({ }^{(\mathrm{Pr} P D I}\right) \mathrm{Fe}\left(\mathrm{N}_{2}\right)_{2}$ & $24 \mathrm{~h}$ & $0 \%$ \\
\hline & $\left({ }^{\mathrm{Me}} \mathrm{PDI}\right) \mathrm{Fe}\left(\mathrm{N}_{2}\right)_{2}$ & $24 \mathrm{~h}$ & $2 \%$ \\
\hline & $\left({ }^{(\mathrm{Pr}} \mathrm{CNC}\right) \mathrm{Fe}\left(\mathrm{N}_{2}\right)_{2}$ & $24 \mathrm{~h}$ & $20 \%$ \\
\hline & $\left({ }^{\mathrm{Mes}} \mathrm{CNC}\right) \mathrm{Fe}\left(\mathrm{N}_{2}\right)_{2}$ & $1 \mathrm{~h}$ & $>95 \%$ \\
\hline & $\left({ }^{(\mathrm{Pr}} \mathrm{CNC}\right) \mathrm{CoMe}$ & $120 \mathrm{~h}$ & $>95 \%$ \\
\hline & $\left({ }^{(\mathrm{Pr} P D I}\right) \mathrm{Fe}\left(\mathrm{N}_{2}\right)_{2}$ & $24 \mathrm{~h}$ & $0 \%$ \\
\hline & $\left({ }^{\mathrm{Me}} \mathrm{PDI}\right) \mathrm{Fe}\left(\mathrm{N}_{2}\right)_{2}$ & $24 \mathrm{~h}$ & $0 \%$ \\
\hline & $\left({ }^{(\mathrm{Pr}} \mathrm{CNC}\right) \mathrm{Fe}\left(\mathrm{N}_{2}\right)_{2}$ & $24 \mathrm{~h}$ & $0 \%$ \\
\hline & $\left({ }^{\mathrm{Mes}} \mathrm{CNC}\right) \mathrm{Fe}\left(\mathrm{N}_{2}\right)_{2}$ & $24 \mathrm{~h}$ & $0 \%$ \\
\hline & $\left({ }^{(\mathrm{Pr}} \mathrm{CNC}\right) \mathrm{CoMe} e^{b}$ & $24 \mathrm{~h}$ & $69 \%$ \\
\hline
\end{tabular}

Pyridyldicarbenes were extensively examined in olefin hydrogenation. Comparison of catalytic activities of iron and cobalt PDI and CNC catalysts in alkene hydrogenation is shown in Table 8. ${ }^{373,374}$ The carbene based pincer catalysts are in general superior particularly for the conversion of branched substrates, like 2-methyl-2-butene or 1-methyl-1cyclohexene. The highly challenging substrate 2,3-dimethyl-2-butene, is only hydrogenated by the cobalt $\mathrm{CNC}$ catalyst. ${ }^{373}$ The excellent performance of the (CNC)Fe catalysts with redox innocent pincer ligands, raises the fundamental question if ligand-mediated single-electron redox behavior can generally be compensated by strong donor ligands that support 2-electron oxidative addition with $3 \mathrm{~d}$ metals. This hypothesis is supported by the facile oxidative addition of silanes $^{489}$ and dihydrogen on the $(\mathbf{C N C}) \mathrm{Fe}\left(\mathrm{N}_{2}\right)_{2}$ (Fe-18) platform ${ }^{490}$ Dinuclear oxidative addition has also been reported. ${ }^{147,149,150}$

The structural related CCC $\left({ }^{\text {Mes }} \mathrm{CCC}=\right.$ bis(mesityl-benzimidazol-2-ylidene)phenyl)) cobalt complexes have been reported by Fout to be efficient catalysts in alkene ${ }^{491}$ and alkyne $^{492}$ hydrogenation (Scheme 67). Remarkably, reactive polar substrates like aldehydes or alcohols could be efficiently converted The catalyst was selective for terminal alkenes as displayed for isoprene where the geminal olefin stayed intact. On the basis of PHIP (parahydrogen induced polarization) transfer NMR spectroscopy studies, a mechanism is suggested which starts with the oxidative addition of dihydrogen. In the presence of olefin, the olefin coordinates to the metal and inserts into the metal hydride bond. Like in the example before it seems strong donating ligands undergo facile oxidative addition. This catalyst was furthermore active in alkyne hydrogenation to selectively form the trans product via cis-hydrogenation followed by an isomerization step. Similiar to the former example, a cobalt(I)/(III)couple is operating. ${ }^{492}$

Scheme 67. Alkene Hydrogenation ${ }^{491}$ and Alkyne Semihydrogenation ${ }^{492}$ with a Cobalt CCC Pincer Complex Co-34. Conversions determined by ${ }^{1} \mathrm{H}$ NMR spectroscopy.

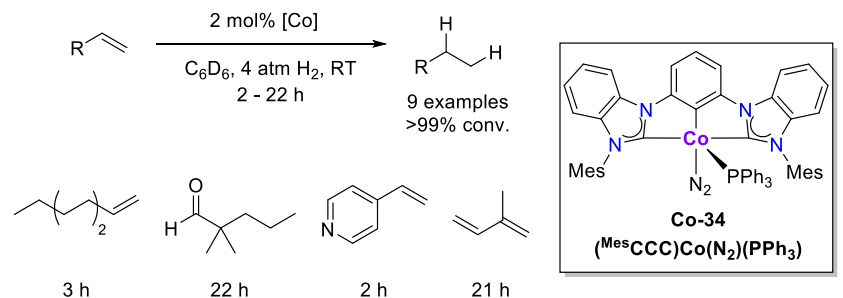

Chirik and co-workers established a $C_{l}$ symmetric pincer platform for asymmetric, cobalt catalyzed hydrogenation of $\alpha$-isopropylstyrenes (Scheme 68), ${ }^{296}$ reminiscent of Bianchini's catalyst for ethylene oligomerization. ${ }^{270}$ The precatalyst is obtained by methylation of the corresponding chloro compound with MeLi. Interestingly, alkylation with neosilyl lithium leads to pincer cyclometalation, which is reversible under an atmosphere of dihydrogen generating as alternative access to the active cobalt monohydride. ${ }^{299}$ Substrates with both electron donating and withdrawing groups as well as sterically demanding substrates were hydrogenated in high yield. Electron withdrawing substituents reduce the stereoselectivity. 
Scheme 68. Hydrogenation of prochiral olefins with catalyst $S$ ( ${ }^{\text {Dipp PDI }}{ }^{\mathbf{C y}, \mathbf{M e}}$ )CoMe (a: Yields are determined to isolated substance and GC, ee's determined by GC. ${ }^{296,299}$

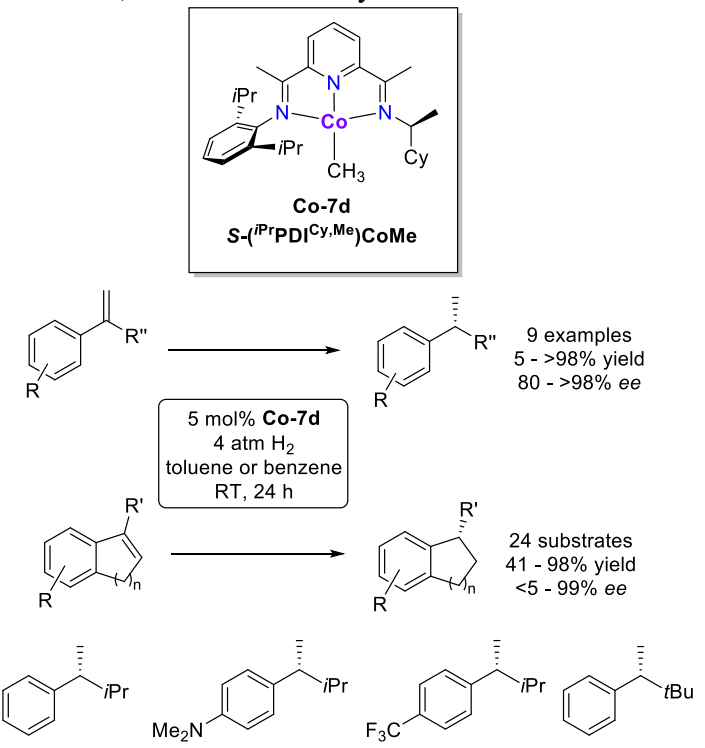

$87 \%(90 \%, R) \quad>98 \%(96 \%, R) \quad>98 \%(66 \%, R) \quad 5 \%(98 \%, R)$

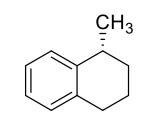

$88 \%, 95 \% R-(-)$ $96 \%, 93 \% R-(-)$
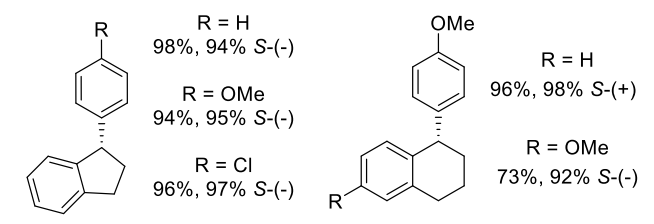

This catalyst was further utilized for enantioselective hydrogenation of unactivated, cyclic alkenes like dialins, 1,1-diarylethenes and 1,2-dihydronaphthalenes (Scheme 68). ${ }^{299}$ These substrates are challenging for enantioselective hydrogenation due to hindered stereoinduction. A total scope of 24 examples was examined with a broadly diversified choice of substrates using $5 \mathrm{~mol} \%$ catalyst loading. Phenyl substituted indenes were reduced almost quantitatively with excellent selectivity and chemical group tolerance. Hydrogenation of 1,2-dihydronaphthalenes also showed high yields and selectivities for the $S$-enantiomer. Reducing the catalyst loading to $0.1 \mathrm{~mol} \%$ did not significantly affect yield or selectivity. Based on stoichiometric model reactions and deuterium labelling studies, Chirik and co-workers proposed a model for stereoinduction: ${ }^{299}$ Prochiral substrates approach the cobalt for stereo determining 1,2-insertion with the smaller substituent oriented towards the more bulky, $C_{1}$ symmetric alkyl imine arm of the pincer ligand.

Stereoselective, cobalt catalyzed olefin hydrogenation was also reported by Chen et al. using a chiral IPO ligand (Scheme 69). The bench stable, divalent cobalt(II) precursor is in situ activated by $\mathrm{NaBEt}_{3} \mathrm{H} .{ }^{384} \mathrm{~A}$ total scope of 321,1 diarylethenes as substrates was examined. Yields are generally high and high stereoselectivities were obtained for bulky and electron withdrawing substrates. ${ }^{384}$
Scheme 69. Stereoselective hydrogenation of 1,1-diarylethenes catalyzed by a cobalt IPO complex. ${ }^{384}$

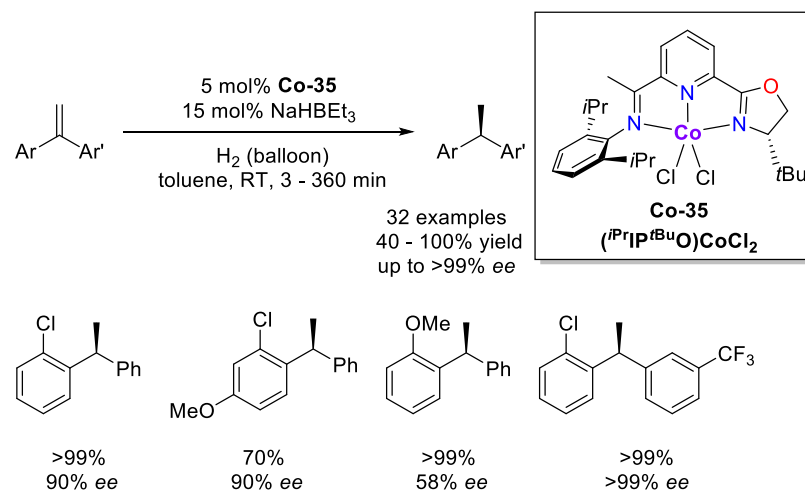

\subsubsection{Aminodiphosphine and Related Pincer}

Catalysts Besides ketones (Section 3.1.), imines (Section 3.2.), $N$-heterocycles (Section 3.2.) and esters (Section 3.5.), first and foremost olefins are hydrogenated by Hanson's catalyst at mild conditions (Scheme 31). Activation of low-spin cobalt(II) precatalyst Co-15a with Brookhart's acid enabled the hydrogenation of a broad range of 12 terminal and 1,2disubstituted aromatic and aliphatic olefins using $2 \mathrm{~mol} \%$ catalyst loading at mild conditions $\left(1 \mathrm{~atm} \mathrm{H}_{2}\right.$, r.t., $\left.24 \mathrm{~h}\right) .{ }^{195}$ This work was extended for olefin transfer hydrogenation by Zhang and coworkers using iso-propanol as the hydrogen source. ${ }^{493}$ A substrate scope of 14 alkenes were hydrogenated with $2 \mathrm{~mol} \% \mathrm{Co}-15 \mathrm{a} /\left[\mathrm{H}\left(\mathrm{Et}_{2} \mathrm{O}\right)_{2}\right] \mathrm{BAr}_{4}{ }_{4}$ at $100{ }^{\circ} \mathrm{C}$ in isopropanol/THF. The Hanson group also utilized the nickel(II) aminopincer hydride complex Ni-15 as a catalyst in the hydrogenation of alkenes (Scheme 70). ${ }^{201}$ With $10 \mathrm{~mol} \%$ of Ni15, a range of five alkenes could be hydrogenated. However, yields were significantly lower in the presence of other reducible groups like aldehydes.

Scheme 70. Olefin hydrogenation with precatalyst Ni-15 (a: Yields determined by ${ }^{1} \mathrm{H}$ NMR spectroscopy). ${ }^{201}$

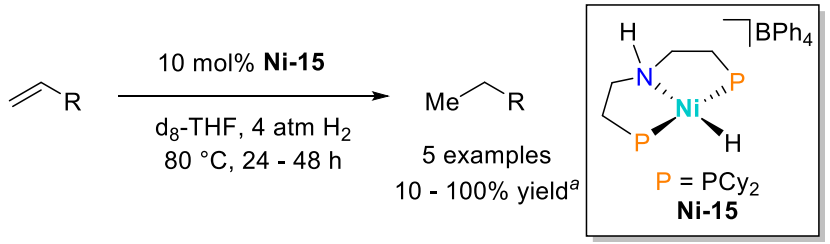

The Hanson group reported several control experiments to gain information about the mechanism of $\mathrm{Co}$ - and $\mathrm{Ni}$-catalyzed olefin hydrogenation. ${ }^{195,426}$ As pointed out above (Section 3.1.2.), the crystallographic characterization of the protonated cobalt(II) precursor remains ambiguous leaving the exact nature of this species unclear. ${ }^{195}$ In continuation, the authors propose the formation of a cobalt(II) monohydride from hydrogenolysis of the protonated precursor, supported by observation of $\mathrm{SiMe}_{4}$ and hydride trapping chloroform. Rapid olefin isomerization in the absence of $\mathrm{H}_{2}$ and $\mathrm{H} / \mathrm{D}$ scrambling crossover experiments further sustain the proposal of an active cobalt hydride species. Furthermore, in contrast to Co-catalyzed ketone reduction, the $\mathrm{N}$-methylated precursors Co-15Me and Ni-15Me exhibit similar catalytic activities as the protonated congeners indicating no 
involvement of MLC in alkene hydrogenation. Based on these observations, monohydride inner-sphere mechanisms for alkene hydrogenation were proposed for both the cobalt and nickel system (Scheme 71). ${ }^{201,426}$ In case of the cobalt system, the proposed mechanism is in agreement with a computational study. ${ }^{494}$ This work also showed a much higher barrier for hydrogenolysis of the neutral cobalt(II) alkyl complex $\operatorname{Co}(n \operatorname{Pr})\left({ }^{\mathrm{iPr} P N P}\right)$ as an explanation for the necessity of the acid co-catalyst.

Scheme 71. Proposed Mechanism for the Cobalt and Nickel Pincer Catalyzed Alkene Hydrogenation $\left(\mathrm{P}=\mathrm{PCy}_{2}\right) .{ }^{201,426}$

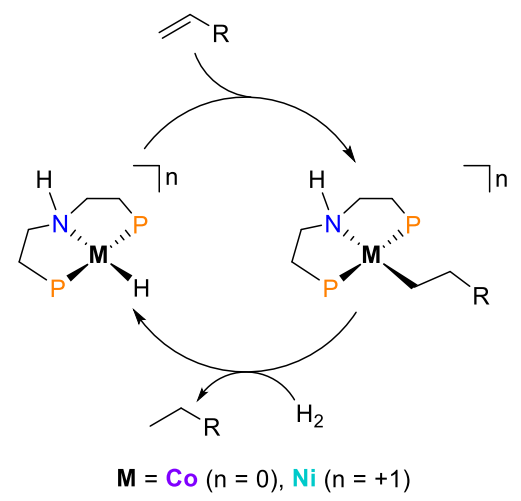

Hydrogenation of a range of 11 olefin C-C double bonds with the amide catalyst ${ }^{i P r} \mathbf{F e}-15 a$ was reported by $\mathrm{Xu}$ and coworkers. ${ }^{196}$ Notably, under the applied conditions (5 mol\% ${ }^{i P r} \mathbf{F e}-15 a, 1$ atm $\mathrm{H}_{2}$, r.t.) chemoselective alkene hydrogenation in presence of esters, nitriles and $N$-heterocycles is possible, which require higher temperatures and pressures with this catalyst, as discussed in Sections 3.5 and 3.2., respectively. However, $\alpha, \beta$-unsaturated ketones are fully reduced to the saturated alcohols. Hammett-analysis of parasubstituted styrenes showed strong rate acceleration for electron withdrawing substrates. Furthermore, stoichiometric styrene hydrogenation was observed for in situ generated $\left({ }^{i}{ }^{\mathbf{P r}} \mathbf{P N}{ }^{\mathrm{H}} \mathbf{P}\right) \mathrm{Fe}(\mathrm{H})_{2} \mathrm{CO}$, yet no conversion for the backbone methylated precursor $\left({ }^{i \mathbf{P r}} \mathbf{P N}{ }^{\mathrm{Me}} \mathbf{P}\right) \mathrm{Fe}\left(\mathrm{BH}_{4}\right) \mathrm{H}(\mathrm{CO})$. These observations lead to the proposal of a stepwise outer-sphere mechanism for hydrogen transfer to the substrate. This pathway is analogous to ketone hydrogenation with this platform, yet rather unusual for olefin hydrogenation.

Scheme 72. Alkene hydrogenation with precatalyst ${ }^{i \operatorname{Pr}} \mathbf{F e - 1 5 a}$ (a: reduction to 4-phenylbutane-2-ol). ${ }^{196}$

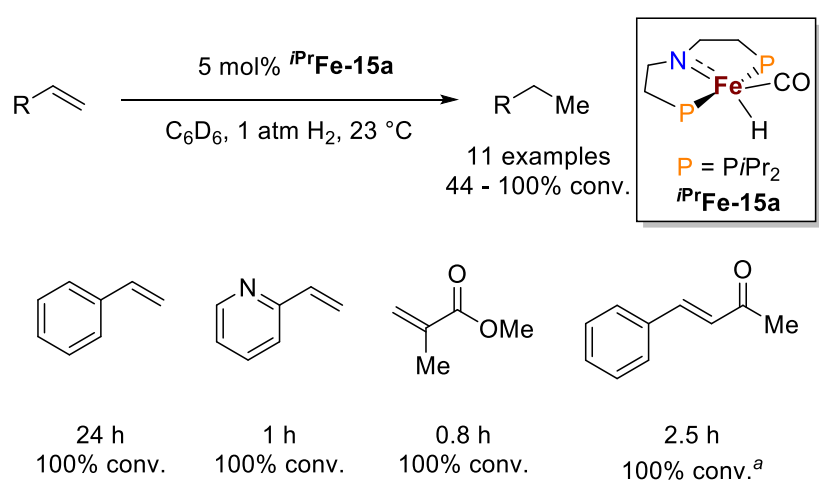

Peters and co-workers reported the unusual PBP cobalt(I) boryl complex Co-6a as a catalyst for rapid styrene hydrogenation with low catalyst loading at mild conditions (Scheme 73). ${ }^{495}$ The turnover frequency $\left(1000 \mathrm{~h}^{-1}\right)$ compares well with Chirik's iron benchmark catalyst $\left({ }^{\mathbf{P P r} P D I}\right) \mathrm{Fe}\left(\mathrm{N}_{2}\right)_{2}$ $\left(1344 \mathrm{~h}^{-1}\right) .{ }^{298}$ Co-6a reversibly adds $\mathrm{H}_{2}$ leading to the dihydrido hydroborate complex Co-6b.

Scheme 73. Styrene hydrogenation and stoichiometric $\mathrm{H}_{2}$ activation by Co-6a. ${ }^{495}$
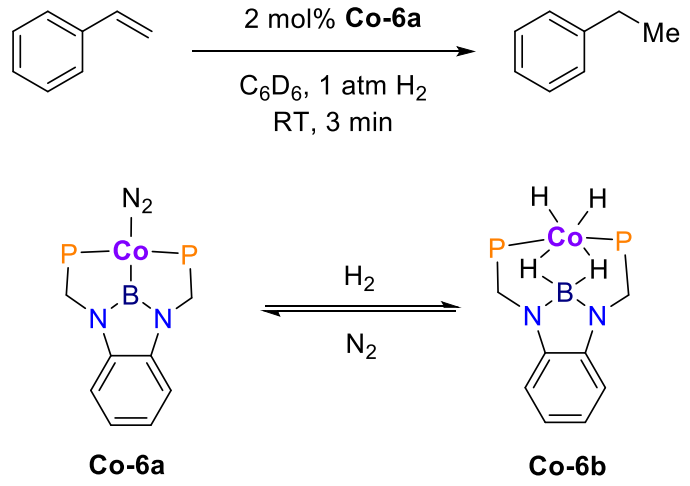

A related nickel(0) PBP borane catalyst for styrene hydrogenation was also reported. ${ }^{496}$ Subsequent detailed computational examination favors a novel inverse MLC pathway for $\mathrm{H}_{2}$ heterolysis with hydride transfer to the functional Ztype borane pincer and protonation of the metal (Scheme 74). ${ }^{497}$

Scheme 74. Catalytic hydrogenation of olefins with $\mathrm{PBP} \mathrm{Ni}(0)$ pincer complex Ni-36a and reversible $\mathrm{H}_{2}$ activation. ${ }^{496}$
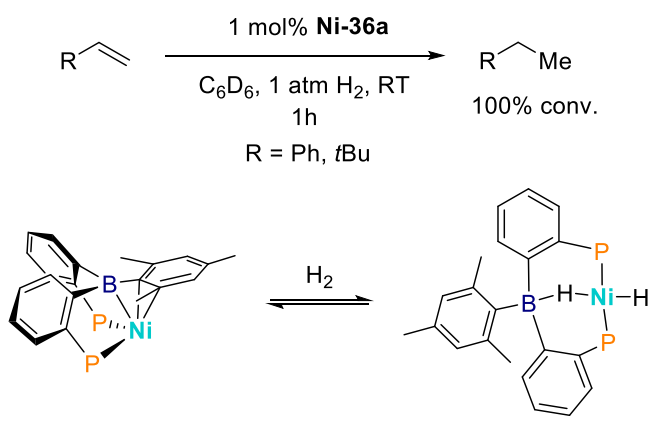

$\mathrm{Ni}-36 \mathrm{a}$

$\mathrm{Ni}-36 \mathrm{~b}$ 


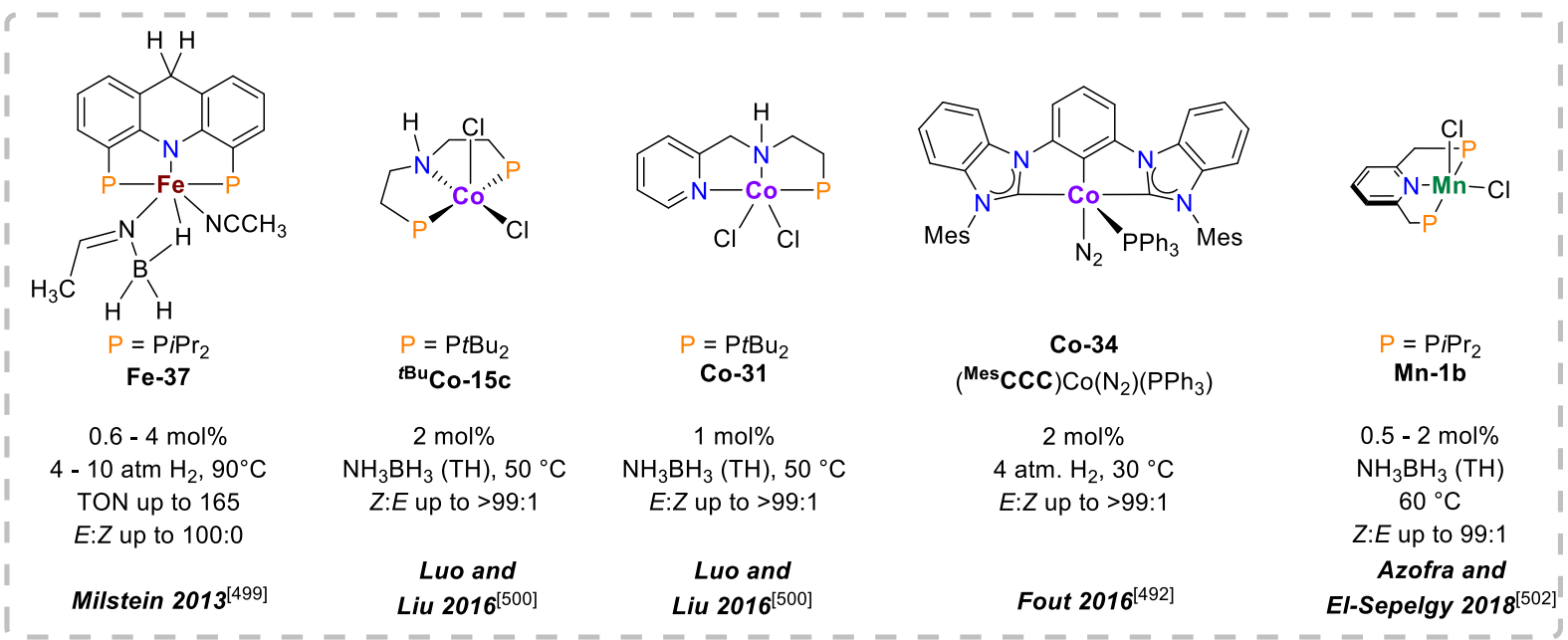

Figure 39. Summary of alkyne semihydrogenation catalysts based on PNP, CCC and PNN pincer ligands.

\subsection{Hydrogenation of Alkynes}

Common protocols for semihydrogenation of alkynes to $Z$ alkenes apply Lindlar's catalyst, while the selective hydrogenation to $E$-alkenes is far less established despite significant progress in recent years. ${ }^{498}$ Milstein and coworker reported an iron dihydroacridine pincer catalyst for mild alkyne semihydrogenation with high $E$-selectivity (Scheme 75). ${ }^{499}$ With 2 mol\% $\%$ Fe-37 predominantly substituted diphenylacetylenes were hydrogenated in almost quantitative yields and high $E / Z$-selectivity.

The groups of Luo and Liu utilized cobalt(II) PNP aminopincer precatalysts for stereodivergent transfer hydrogenation of alkynes with ammonia borane as hydrogen source (Scheme 76). ${ }^{500}$ With $2 \mathrm{~mol} \%$ of ${ }^{t \mathrm{Bu}} \mathbf{C o - 1 5 c}$ alkynes were converted to the respective $Z$-alkenes with predominantly good stereoselectivities and yields. In contrast, the PNN precatalyst Co-31 gave alkenes with inverted stereoselectivity in overall good yields. Based on deuterium-labeling experiments the authors propose cobalt(I) hydride species as the active, $Z$-selective hydrogenation catalysts. However, in case of $\mathbf{C o - 3 1}$, the $Z$-alkene is subsequently isomerized by this species to the $E$-alkene product.

Scheme 75. Catalytic $E$-selective hydrogenation of alkynes with dihydroacridine-based Iron pincer complex Fe-37. ${ }^{499}$
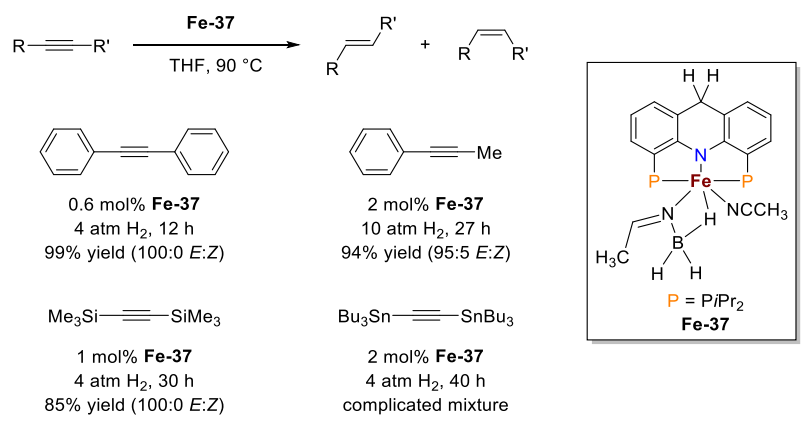

A related cobalt(II) pyridyl pincer catalyst with $N$-morpholine substituted arms was recently applied in the $Z$-selective semihydrogenation of alkynes. ${ }^{501}$ With aminoborane as the dihydrogen source 21 alkynes were reduced with predominantly high stereoselectivity. At higher temperatures, terminal alkenes are further hydrogenated to alkanes.

Scheme 76. Stereodivergent transfer semihydrogenation of alkynes with cobalt pincer precatalysts. ${ }^{500}$

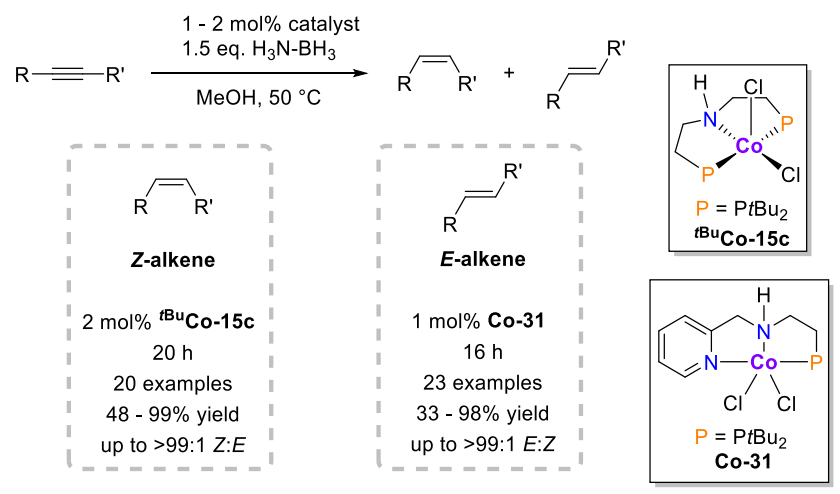

In one recent report the use of an aromatized pyridyl pincer $\mathrm{Mn}(\mathrm{II})$ complex Mn-1b in the transfer semihydrogenation of alkynes with ammonia borane $\left(60-70{ }^{\circ} \mathrm{C}, \mathrm{MeOH}, 20 \mathrm{~h}\right)$ as the hydrogen source is described, however, the authors propose an inner-sphere hydrogenation mechanism without metal-ligand cooperativity. ${ }^{502}$

\section{Dehydrogenation}

\section{1. (Acceptorless) Alcohol Dehydrogenation}

4.1.1. Dialkylamino Pincer Catalysts Acceptorless alcohol dehydrogenation (AAD) is the oxidant-free elimination of $\mathrm{H}_{2}$ from alcohols giving ketones or esters in the absence of further reagents. Besides the synthetic utility the hydrogen released from (bio)accessible alcohols can be the actual target in the context of chemical hydrogen storage. ${ }^{503-}$ 
${ }^{505}$ Methanol is of particular interest due to the high gravimetric hydrogen content $(12.6 \%)$ and easy transportation. The first report on AAD of methanol with an iron pincer catalyst was reported by Beller and coworkers (Scheme 77). ${ }^{506}$ With loadings of aminopincer catalyst ${ }^{i \mathbf{P r}} \mathbf{F e - 1 5 g}$ as low as $4.5 \mathrm{ppm}$ aqueous phase reforming (APR) of methanol at $91.0{ }^{\circ} \mathrm{C}$ under basic conditions gave $\mathrm{H}_{2}$ and $\mathrm{CO}_{2}$ with a turnover number of 9834 after 46 hours. In analogy to catalytic $\mathrm{CO}_{2}$ hydrogenation, which is discussed in Section 3, the iron amide complex ${ }^{i \mathbf{P r}} \mathbf{F e - 1 5 a}$ is proposed as active species within an all-iron(II) mechanism. Subsequent improvement was achieved by Bielinski et al. employing the PNP formate complex $\left[\left({ }^{i{ }^{P r}} \mathbf{P N}{ }^{H} \mathbf{P}\right) \mathrm{Fe}(\mathrm{OC}(\mathrm{O}) \mathrm{H}) \mathrm{H}(\mathrm{CO})\right]\left({ }^{\mathbf{i P r}} \mathbf{F e}-15 \mathbf{h}\right)$ and Lewis acid $\mathrm{LiBF}_{4}$ as co-catalyst, which resulted in a TON up to 51000 (Scheme 77). ${ }^{507}$

Scheme 77. Aqueous phase reforming of methanol with iron pincer catalysts ${ }^{i \text { Pr Fe-15g and }{ }^{i \mathbf{P r}} \mathbf{F e}-15 \text { h. }}{ }^{506,507}$

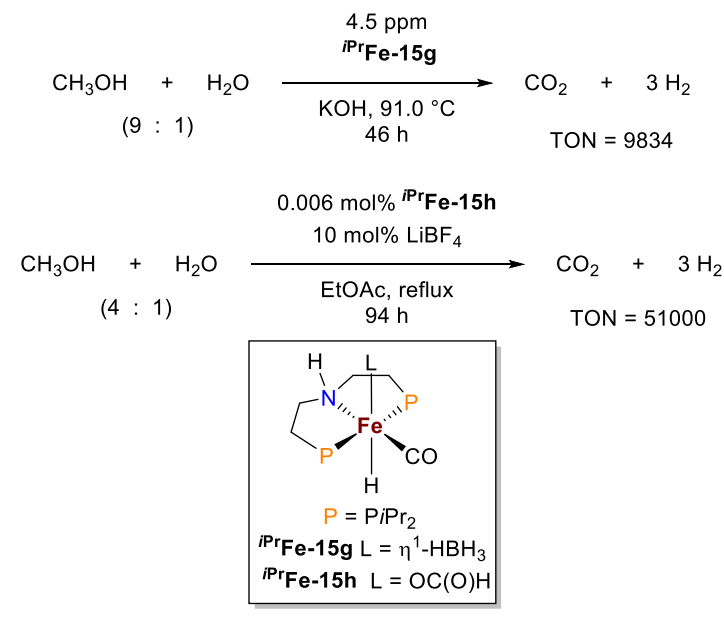

A mechanism supported by DFT analysis was proposed via 1) catalytic $\mathrm{MeOH}$ dehydrogenation formaldehyde, 2) hydrolysis of formaldehyde to methanediol, 3) dehydrogenation of methanediol to the iron formate complex ${ }^{i \mathbf{P r}} \mathbf{F e}-\mathbf{1 5 h}$ and 4) Lewis-acid facilitated decarboxylation of ${ }^{i P r} \mathbf{F e}-15 h .{ }^{507}$ The mechanistic details for alcohol and formate dehydrogenation are identical with the respective forward reactions and were discussed there. Once again, MLC reactivity of this platform plays a pivotal role for $\mathrm{H}_{2}$ elimination steps from the iron(II) dihydride amine species. In the absence of water methyl formate is obtained from dehydrogenation of a hemiacetal intermediate.

Scheme 78. AAD of $2^{\circ}$ and $1^{\circ}$ alcohols catalyzed by iron pincer Precatalyst ${ }^{\text {iPr }}$ Fe-15g. 424

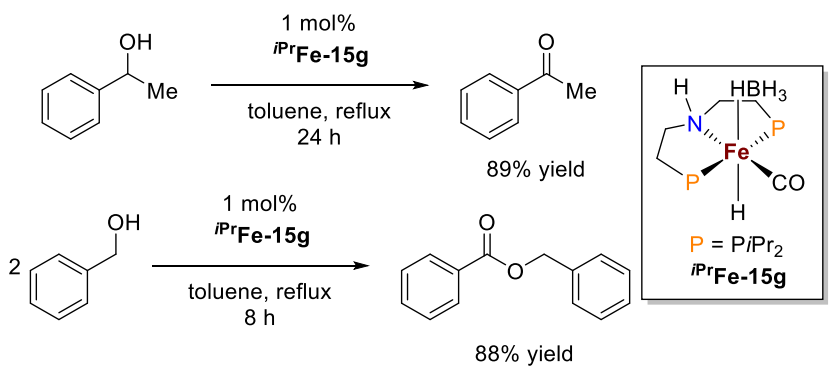

AAD of a broad range of primary and secondary alcohols to ketones ( $2^{\circ}$ alcohols) and esters ( $2^{\circ}$ alcohols), respectively, was reported by Hazari, Holthausen, Jones and Schneider (Scheme 78), with low loadings in ${ }^{i P r} \mathbf{F e}-15 g$ or ${ }^{i P r} \mathbf{F e}-15 a$ (0.1-1 mol\%) under base free conditions. ${ }^{42,508}$ Lactones were obtained from diol substrates. High temperatures are required (refluxing toluene or THF) to drive the endothermic reaction by $\mathrm{H}_{2}$ removal. DFT calculations for ester formation support an analogous mechanism as for methanol APR, yet with iron catalyzed formation of a hemiacetal that leads to the final ester product.

Subsequently, Hazari and coworkers utilized ${ }^{i \mathbf{P r}} \mathbf{F e}-\mathbf{1 5 g}$ for the dehydrogenation of glycerol to lactic acid salts. ${ }^{509}$ In the presence of stoichiometric $\mathrm{NaOH}$ TON of 1050 was achieved in $N$-methyl-2-pyrrolidinone/water (1:1). The proposed mechanism is shown in Scheme 79.

Scheme 79. AAD of glycerol to sodium lactide with precatalyst ${ }^{i \operatorname{Pr}} \mathbf{F e}-15 \mathrm{~g}(\mathrm{~A})$ and proposed mechanism $(\mathbf{B})\left(\mathrm{P}=\mathrm{P} i P r_{2}\right) .{ }^{509}$

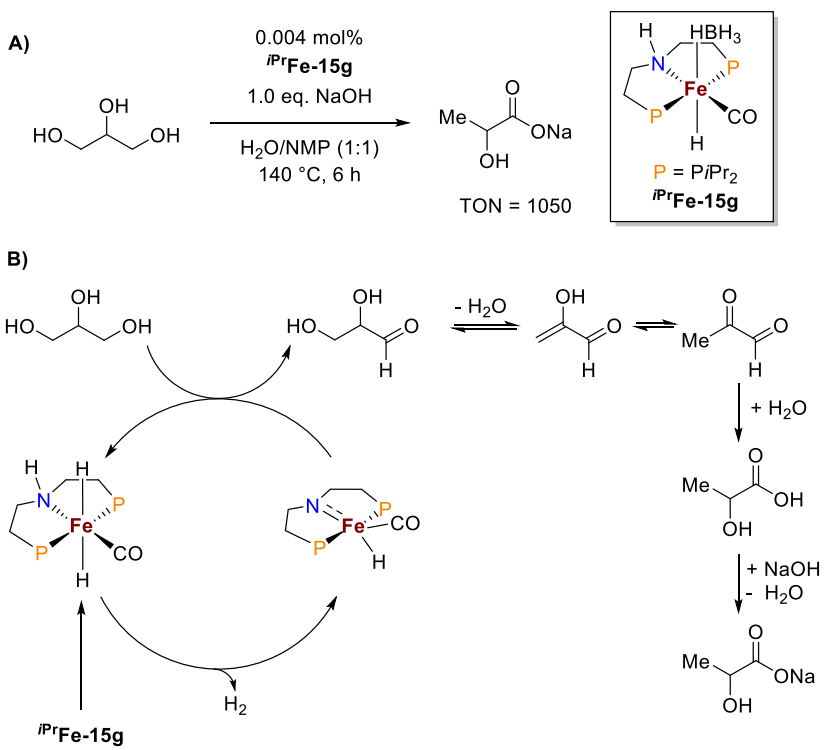

The use of glycerol AAD as a sustainable hydrogen source allowed for acetophenone transfer hydrogenation to 1-phenylethanol under basic conditions. ${ }^{509}$ 


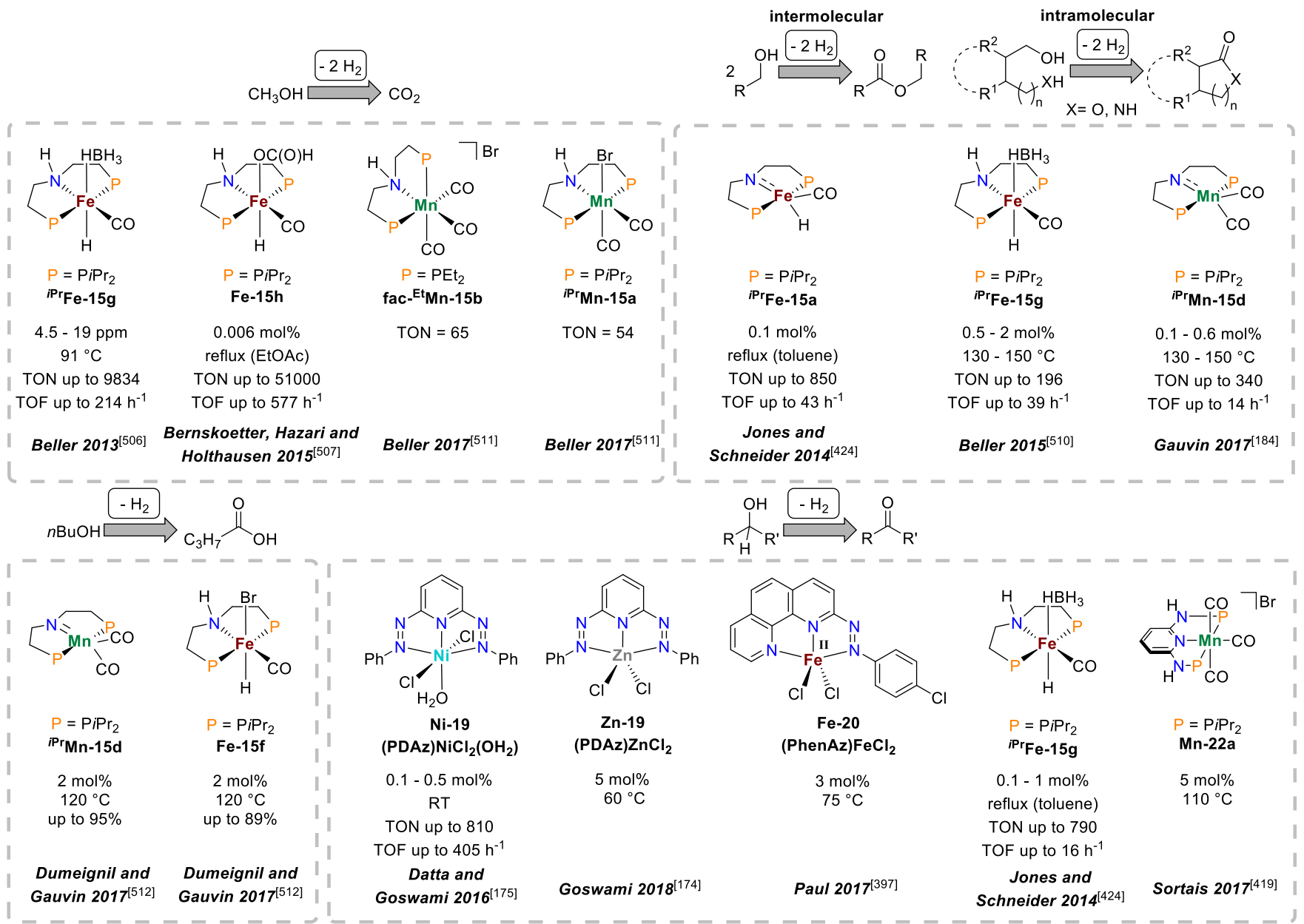

Figure 40. Summary of acceptorless alcohol dehydrogenation catalysts based on PNP dialkylamino pincer ligands.

Table 9. Dehydrogenation of diols to lactones with precatalyst

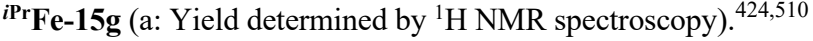

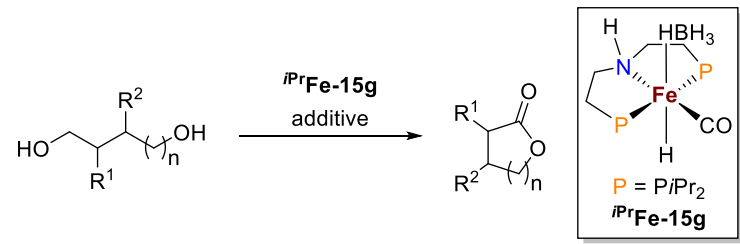

\begin{tabular}{|c|c|c|c|c|c|c|}
\hline diol & $\begin{array}{c}\text { cat. } \\
\text { loading }\end{array}$ & solvent & additive & $\begin{array}{c}\text { temp. } \\
{\left[{ }^{\circ} \mathrm{C}\right]}\end{array}$ & $\begin{array}{c}\text { time } \\
{[\mathrm{h}]}\end{array}$ & $\begin{array}{c}\text { yield } \\
{[\%]}\end{array}$ \\
\hline & $0.5 \mathrm{~mol} \%$ & $\begin{array}{l}t \text {-amyl } \\
\text { alcohol }\end{array}$ & $\begin{array}{c}\mathrm{K}_{2} \mathrm{CO}_{3} \\
(10 \mathrm{~mol} \%)\end{array}$ & 150 & 5 & 92 \\
\hline & $1 \mathrm{~mol} \%$ & toluene & - & 120 & 8 & 96 \\
\hline & $0.5 \mathrm{~mol} \%$ & $\begin{array}{l}t \text {-amyl } \\
\text { alcohol }\end{array}$ & $\begin{array}{c}\mathrm{K}_{2} \mathrm{CO}_{3} \\
(10 \mathrm{~mol} \%)\end{array}$ & 150 & 5 & 82 \\
\hline & $1 \mathrm{~mol} \%$ & $\begin{array}{l}t \text {-amyl } \\
\text { alcohol }\end{array}$ & $\begin{array}{c}\mathrm{K}_{2} \mathrm{CO}_{3} \\
(10 \mathrm{~mol} \%)\end{array}$ & 150 & 5 & $55^{a}$ \\
\hline & $1 \mathrm{~mol} \%$ & toluene & - & 120 & 24 & 59 \\
\hline
\end{tabular}

In continuation of the work of Jones and Schneider, ${ }^{424}$ Beller and co-workers reported the synthesis of several lactones by AAD of diols with borohydride complex ${ }^{i P r} \mathbf{F e}-15 g(0.5-1$ $\mathrm{mol} \%$ ) as catalyst in moderate to excellent yields (Table 9). ${ }^{510} \mathrm{~A}$ three-step pathway with two catalytic dehydrogenation steps connected by intramolecular lactol cyclization was proposed (Scheme 80). Both catalytic AAD reactions were suggested to follow all-iron(II) amide/amine hydride cycles as previously proposed. ${ }^{424}$ 
Scheme 80. Proposed mechanism for dehydrogenative lactonization of diols $\left(\mathrm{P}=\mathrm{PiPr}_{2}\right){ }^{424,510}$

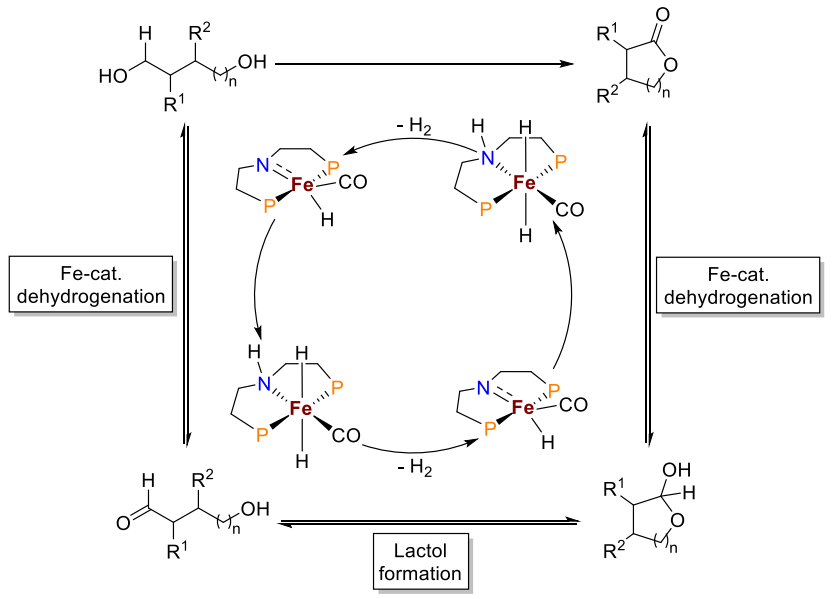

Beller and coworkers introduced the manganese dicarbonyl amine precatalyst ${ }^{i P r}$ Mn-15a to methanol APR (Scheme 81). ${ }^{511}$ Under basic aqueous conditions a turnover number of 54 was obtained after 5 hours. Interestingly, the cyclohexyl substituted derivative ${ }^{\mathbf{C y}} \mathbf{M n - 1 5 a}$ was inactive. Slightly higher TON were achieved with the tricarbonyl precursor fac- ${ }^{\mathrm{Et}} \mathrm{Mn-15b}\left(\mathrm{TON}_{5 \mathrm{~h}}=65\right)$ and a mixture of $\mathrm{MnBr}(\mathrm{CO})_{5}$ and ${ }^{i \operatorname{Pr}} \mathbf{P N}{ }^{\mathrm{H}} \mathbf{P}$ (10 equiv.), respectively $\left(\mathrm{TON}_{5 \mathrm{~h}}=68\right),{ }^{i \operatorname{Pr}} \mathbf{M n}-$ 15a exhibited remarkable long-term stability $(>1$ month; TON >20000) in presence of excess of ${ }^{i \mathbf{P r}} \mathbf{P} \mathbf{N}^{\mathbf{H}} \mathbf{P}$. Based on stoichiometric experiments the authors proposed in situ formation of five-coordinate amide complex ${ }^{\mathbf{R}} \mathbf{M n - 1 5 d}$ as active species that adds methanol and eliminates formaldehyde via a MLC pathway. Subsequent $\mathrm{H}_{2}$ loss from the hydride product ${ }^{\mathbf{R}} \mathbf{M n}-\mathbf{1 5}$ c was shown computationally for a related system. ${ }^{444}$

Scheme 81. APR of methanol with $\left({ }^{\mathbf{R}} \mathbf{P N}{ }^{\mathrm{H}} \mathbf{P}\right) \mathrm{Mn}$ catalysts. ${ }^{511}$
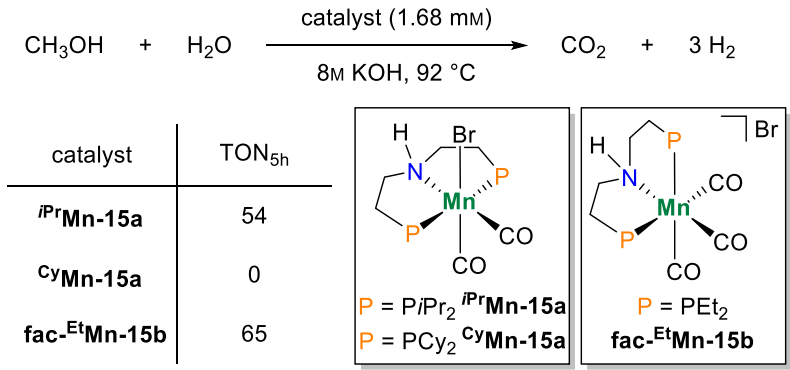

In a related study, Gauvin and co-workers directly utilized the five-coordinate amide complex ${ }^{i \mathbf{P r}} \mathbf{M n - 1 5 d}$ in AAD for the synthesis of 18 benzylic and aliphatic esters in moderate to excellent yields (Scheme 82). ${ }^{184}$ Yields strongly depend on substrate sterics. No reaction was observed for neo-pentyl alcohol.

Reversible addition of the benzylic alcohol to the catalyst was spectroscopically observed. The alkoxide product forms the respective monohydride complex ${ }^{i \mathbf{P r}} \mathbf{M n - 1 5 c}$ (and ester) upon heating. Computational treatment of the dehydrogenation pathway gave an analogous mechanistic picture as for the iron catalyst ${ }^{i P r} \mathbf{F e}-15 \mathbf{a},{ }^{424}$ with an all-manganese(I) amide/amine hydride MLC cycle, as previously proposed by
Beller for this system. ${ }^{511}$ Accordingly, the $N$-methylated hydride complex ( $\left.{ }^{i \mathbf{P r}} \mathbf{P} \mathbf{N}^{\mathrm{Me}} \mathbf{P}\right) \mathrm{MnH}\left(\mathrm{CO}_{2}\right)$ exhibits poor catalytic performance. As in the iron case, alcohol assisted $\mathrm{H}_{2}$-elimination from the hydride amine state was computed as turnover limiting barrier.

Scheme 82. Dehydrogenative coupling of alcohols to esters catalyzed by ${ }^{i \operatorname{Pr}} \mathbf{M n - 1 5 d}$ (a: Yields determined by ${ }^{1} \mathrm{H}$ NMR spectroscopy; b: TON $=340$ with $0.1 \mathrm{~mol} \%{ }^{i \text { Pr }}$ Mn-15d). ${ }^{184}$

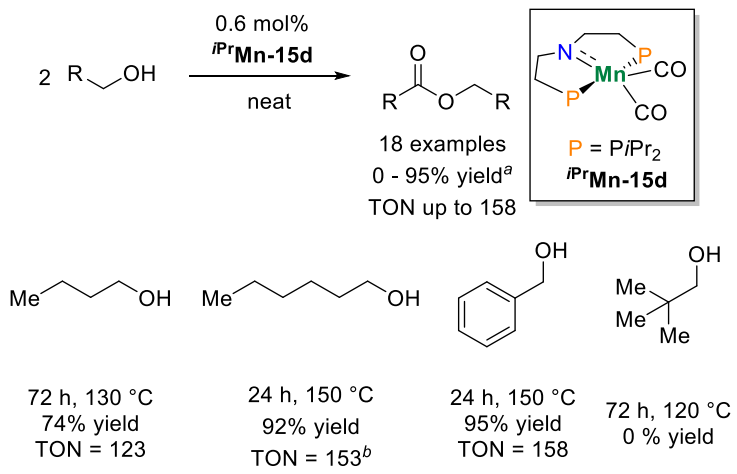

Gauvin and coworkers further screened several ${ }^{\mathbf{R}} \mathbf{P N} \mathbf{N}^{\mathbf{H}} \mathbf{P}$ based $(\mathrm{R}=i \mathrm{Pr}, \mathrm{Cy})$ iron and manganese precursors as catalysts for AAD of 1-butanol (Scheme 83) and some primary fatty alcohols to the respective carboxylic acids. ${ }^{512}$ The selectivity towards carboxylates is maintained by strongly basic aqueous conditions. Using the same reaction conditions ( $2 \mathrm{~mol} \%$ cat. @ $120^{\circ} \mathrm{C}$ in toluene), the five-coordinate amide complex ${ }^{i \operatorname{Pr}} \mathbf{M n}-\mathbf{1 5 d}$ and the borohydride ( ${ }^{\mathbf{P} P} \mathbf{P}-$ $\left.\mathbf{N}^{\mathbf{H}} \mathbf{P}\right) \mathrm{Mn}\left(\mathrm{BH}_{4}\right)(\mathrm{CO})_{2}$ (Mn-15f) exhibited similar performance and proved superior to iron catalysts presumably due to higher robustness under basic conditions.

Scheme 83. AAD of 1-butanol to butyric acid catalyzed by manganese catalysts ${ }^{i P r} \mathbf{M n - 1 5 d}$ and $\mathbf{M n - 1 5 f}{ }^{512}$

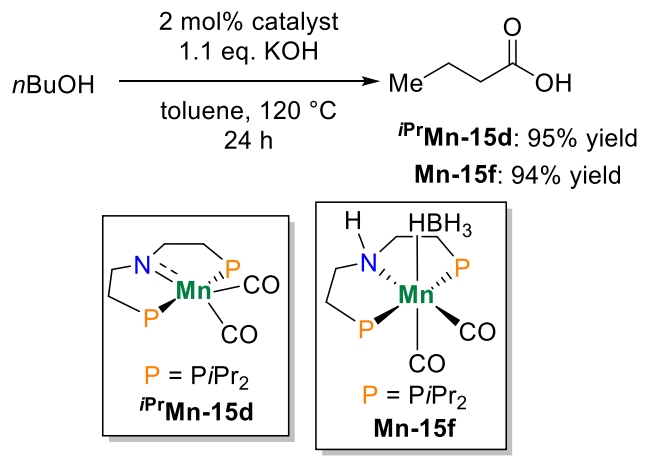

\subsubsection{Pyridyl-Based and related Pincer Cata-}

lysts Sortais utilized the $\mathbf{P N}_{\mathbf{3}} \mathbf{P}$ ligated manganese tricarbonyl complex Mn-22a for AAD of $2^{\circ}$ alcohols to ketones. ${ }^{419}$ Acetophenone was obtained in $84 \%$ yield after $110{ }^{\circ} \mathrm{C}$ in toluene for $22 \mathrm{~h}$. The catalyst loading $(5 \mathrm{~mol} \%)$ was considerably higher compared with the protocol for cat-

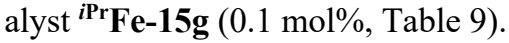

Goswami and coworkers examined aerobic alcohol oxidation using the bisazophenylpyridine pincer catalysts 
(PDAz) $\mathrm{NiCl}_{2}\left(\mathrm{H}_{2} \mathrm{O}\right)$ and (PDAz) $\mathrm{ZnCl}_{2}\left(\mathrm{Scheme}^{84)}{ }^{175}\right.$ Stoichiometric amounts of $\mathrm{KO} t \mathrm{Bu}$ and excess zinc dust are required. Predominantly aryl-substituted primary and secondary alcohols are oxidized with low catalyst loadings (0.1-0.5 mol\%) at mild conditions ( $1 \mathrm{~atm} \mathrm{O}_{2}$, r.t.) in yields between $65-96 \%$ within $2-4 \mathrm{~h}$ for the nickel catalyst. Zinc catalyzed oxidation required higher catalyst loadings ( $5 \mathrm{~mol} \%$ ) elevated temperatures and longer reaction times. ${ }^{174,175}$ The nickel platform was also used for oxidative condensation of aromatic alcohols and hydrazine to phthalazine..$^{513}$

Scheme 84. Aerobic oxidation of primary and secondary alcohols catalyzed by $(\mathbf{P D A z}) \mathrm{NiCl}_{2}\left(\mathrm{H}_{2} \mathrm{O}\right)(\mathbf{N i}-19)$ and $(\mathbf{P D A z}) \mathrm{ZnCl}_{2}(\mathbf{Z n -}$ 19) (a: Yields determined by GC-MS.). ${ }^{174,175}$

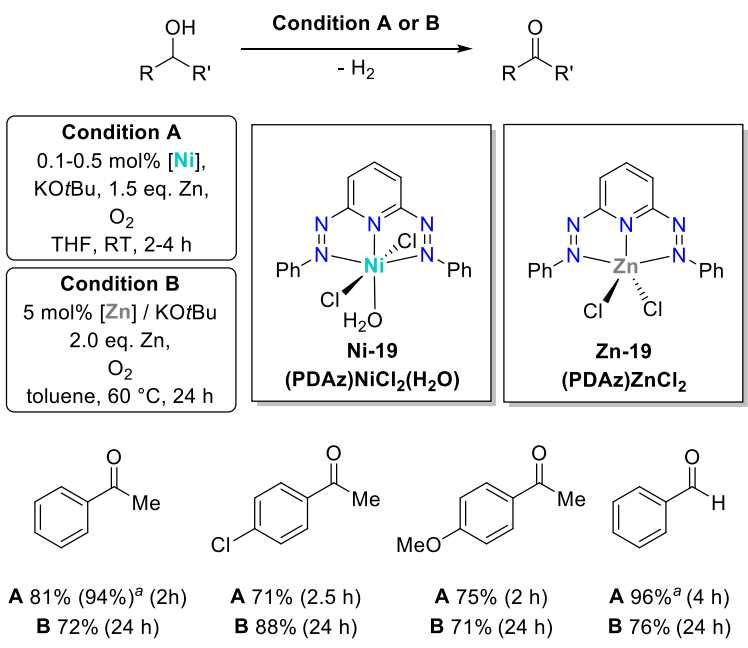

Based on stoichiometric control experiments and DFT studies, a mechanism for aerobic alcohol oxidation was proposed (Scheme 85). Key point of the cycle is that all hydrogen transfer steps are purely ligand based within an all-nickel(II) mechanism. Initial ligand centered 1-electron reduction is supported by in situ EPR spectroscopy. A large H/D kinetic isotope effect was rationalized with a tunneling contribution for turn-over limiting $\mathrm{C}-\mathrm{H}$ hydrogen atom transfer (HAT) from the substrate to a pincer azo-group. After proton transfer from another substrate molecule, the cycle is closed by aerobic pincer re-oxidation. $\mathrm{H}_{2} \mathrm{O}_{2}$ was detected with an iodometric essay and a hydrazo intermediate could be crystallographically characterized. Ligand centered HAT and formation of $\mathrm{H}_{2} \mathrm{O}_{2}$ resemble the enzyme galactose oxidase. ${ }^{514}$ A similar mechanism was postulated for the zinc mediated dehydrogenation. ${ }^{174,175}$ There, a bishydrazo species could be crystallographically characterized.
Scheme 85. Proposed mechanism for nickel mediated aerobic alcohol oxidation (HAT: hydrogen atom transfer; RLS: rate limiting step). ${ }^{175}$
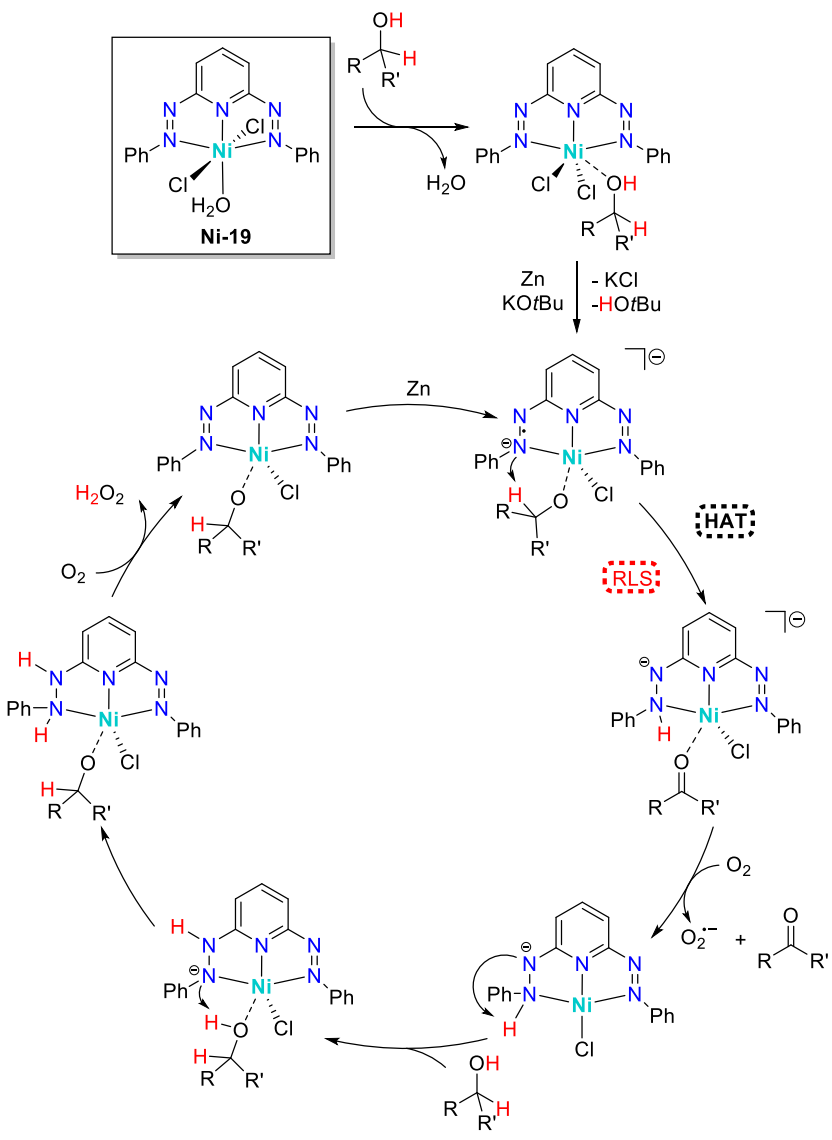

In analogy, a ferrous phenylazophenantroline catalyst was utilized for aerobic oxidation of primary and secondary benzylic alcohols (Scheme 86). ${ }^{397}$ Catalyst activation by reduction with $\mathrm{KO} t \mathrm{Bu}$ was confirmed by EPR spectroscopy which is also known for free phenantroline. ${ }^{515}$ With catalyst loadings of $3.0 \mathrm{~mol} \%$ in toluene @ $75{ }^{\circ} \mathrm{C}$ good to moderate isolated yields were obtained. Electron withdrawing 4-nitrophenyl and pyridyl substituents significantly lower the yield.

Scheme 86. Aerobic oxidation of benzylic alcohols catalyzed by (Phen Az) $\mathrm{FeCl}_{2}$ (isolated yields). ${ }^{397}$

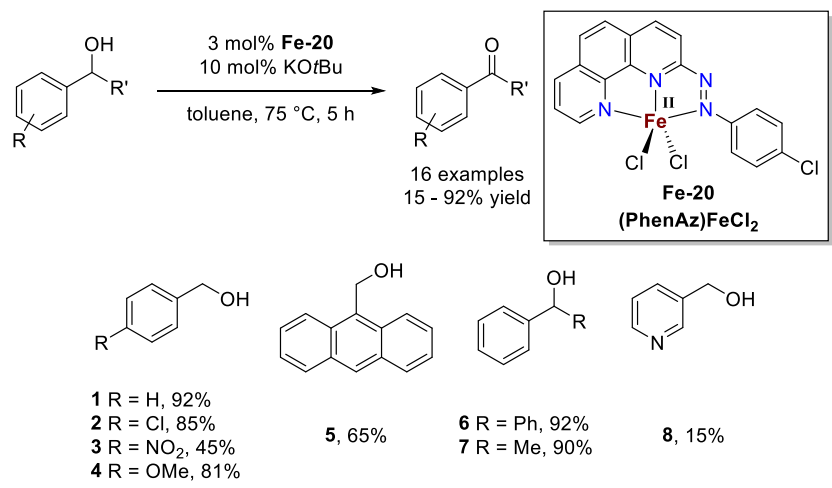

Addition of a radical trap (TEMPO) drastically decelerated conversion in agreement with a radical mechanism. Based on kinetic and spectroscopic data, a mechanism was 
proposed for the alcohol $2 \mathrm{e}^{-} / 2 \mathrm{H}^{+}$oxidation reaction via stepwise HAT from the substrate to the azo unit and subsequently to $\mathrm{O}_{2}$ in analogy to Goswami's nickel catalyst. As a distinguishing feature, the iron catalyst was suggested to undergo one-electron iron(II)/iron(I) redox steps and one-electron step, in contrast with the all-nickel(II) and ligand twoelectron redox cycle. The electronic structures of the putative iron(I) intermediates were not examined and the relevance of phenantroline redox non-innocence ${ }^{515}$ for catalysis needs further clarification (Scheme 87). However, the proton coupled electron transfer (PCET) $)^{516-518}$ reactivity of the azo pincer framework provides a conceptually rare approach to cooperative catalysis, which might open new pathways for challenging substrates.

Scheme 87. Proposed mechanism for (PhenAz) $\mathrm{FeCl}_{2}$-mediated aerobic alcohol oxidation. ${ }^{397}$

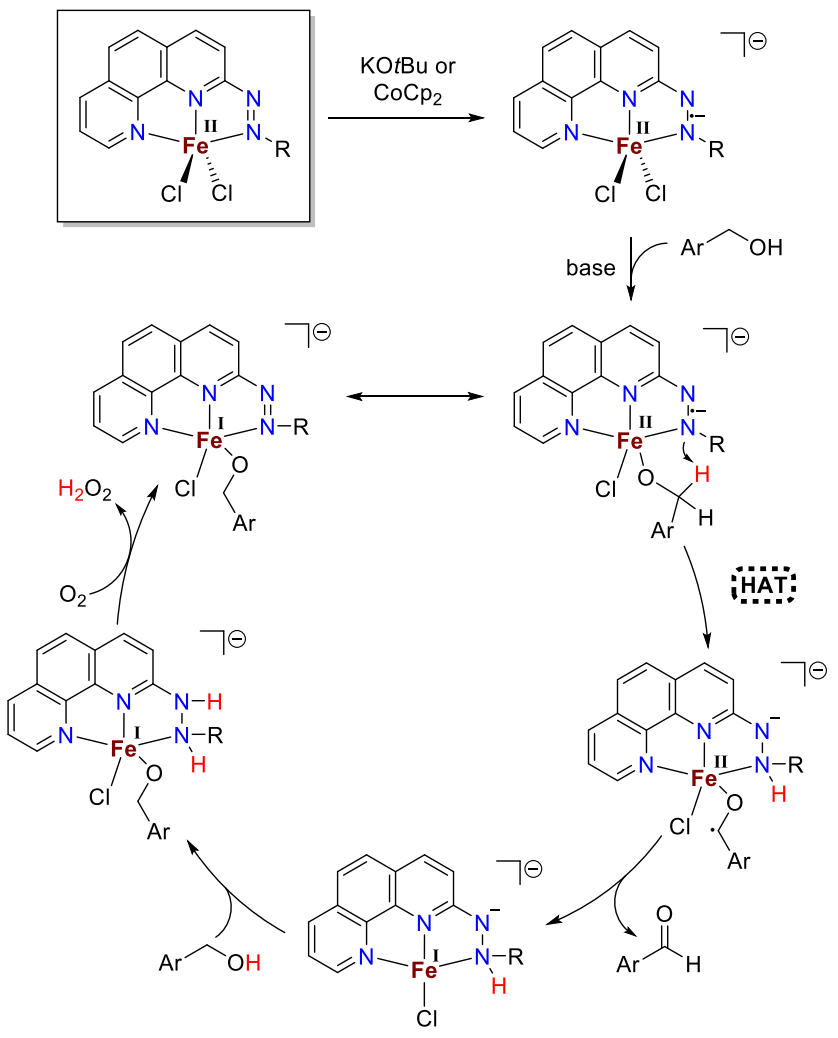

\subsection{Formic Acid Dehydrogenation}

Formic acid is of interest as a molecular hydrogen storage vector due to its high hydrogen content $(4.4 \mathrm{wt} \%)$. Furthermore, the almost thermoneutral $\mathrm{CO}_{2} / \mathrm{HC}(\mathrm{O}) \mathrm{OH}$ de/hydrogenation equilibrium can be easily shifted on either side with $\mathrm{pH}$ enabling a cyclic, overall carbon neutral storage cycle. An important challenge is the suppression of formic acid dehydration to $\mathrm{CO}$, which is a fatal catalyst poison for fuel cells.

Seminal pioneering contributions to $3 \mathrm{~d}$ metal catalyzed formic acid dehydrogenation (FAD) were made by the Beller group. ${ }^{519-521}$ Iron pincer catalysts were first described by Milstein and coworkers. ${ }^{522}$ The dihydride $\left({ }^{\left({ }^{B} \text { u }\right.} \mathbf{P N}{ }^{\mathbf{P y}} \mathbf{P}\right) \mathrm{Fe}(\mathrm{H})_{2} \mathrm{CO}$ catalyzes $\mathrm{FAD}$ at $40{ }^{\circ} \mathrm{C}$ in the presence of base (NEt 3 ) in THF with TOF around $500 \mathrm{~h}^{-1}$ and high long term stability (TON > 100000). Supported by DFT computations, the authors proposed an inner-sphere mechanism without MLC via protonation of the dihydride catalyst and subsequent $\mathrm{CO}_{2}$ extrusion for a formate intermediate. The role of the base was not clarified.

Kirchner, Gonsalvi and co-workers used related iron $\mathbf{P N}_{3} \mathbf{P}$ pincer complexes for selective FAD. ${ }^{523}$ The backbone $N$ protonated (Fe-22a) and $N$-methylated $(\mathbf{F e}-22 \mathrm{Me})$ precursors were examined. Interestingly, Fe-22Me exhibits TON (10000) that are an order of magnitude higher that Fe-22a, rendering a MLC pathway less likely, yet still possible.

Scheme 88. Formic acid dehydrogenation catalyzed by Fe-15h ( $\mathrm{P}$ $\left.=\mathrm{P} \mathrm{Pr}_{2}\right)$. ${ }^{181}$
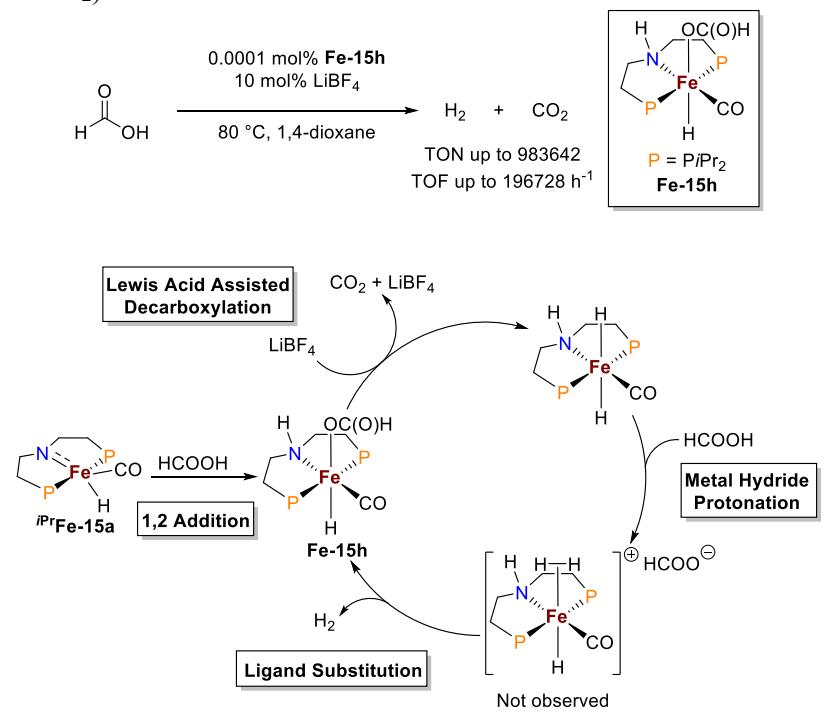

In 2014 the groups of Hazari and Schneider reported a strongly improved catalyst based on the iron diphosphinoamine pincer platform $\left({ }^{\mathrm{R}} \mathbf{P N}{ }^{\mathrm{H}} \mathbf{P}\right) \mathrm{Fe}$ (Scheme 88$) .{ }^{181}$

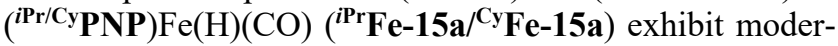
ate catalytic activity. Stoichiometric experiments showed formic acid 1,2-addition giving the formate complex $\mathbf{F e}-\mathbf{1 5 h}$ that can be directly used as a catalyst. Importantly, the authors demonstrated dramatically increased catalytic activity upon addition of Lewis acids. With very low catalyst loadings $(0.0001 \mathrm{~mol} \% \mathbf{F e}-15 \mathbf{h})$ and addition of $10 \mathrm{~mol}_{0} \mathrm{LiBF}_{4}$, unprecedented TOF up to almost $200000 \mathrm{~h}^{-1}$ and catalyst stability (TON around $10^{6}$ ) were obtained. Within a series of Lewis acids, the rate acceleration approximately correlates with carboxylate affinity. In analogy to the systems of Milstein, Kirchner and Gonsalvi, the authors proposed an alliron(II) mechanism without MLC (Scheme 88). The irrelevance of MLC was later supported by the higher activities obtained for the $N$-methylated catalyst ( ${ }^{\mathrm{Cy}} \mathbf{P}$ $\left.\mathbf{N}^{\mathrm{Me}} \mathbf{P}\right) \mathrm{Fe}\left(\mathrm{BH}_{4}\right) \mathrm{H}(\mathrm{CO})$ in reverse $\mathrm{CO}_{2}$ hydrogenation with respect to parent ${ }^{\mathrm{Cy}} \mathbf{F e}-\mathbf{1 5 a}{ }^{475}$ The 'Lewis acid effect' was attributed to stabilization of a $\kappa-\mathrm{H}$ bound formate intermediate $\left(\mathrm{Fe}-\mathrm{H}-\mathrm{CO}_{2} \cdots \mathrm{LA}\right)$ of the rate limiting decarboxylation step. This interpretation could later be confirmed computationally ${ }^{507}$ In fact, this rate acceleration by Lewis acids was shown to apply to several other FAD catalysts but also reverse $\mathrm{CO}_{2}$ hydrogenation as discussed in Section 3.6. ${ }^{33}$ 


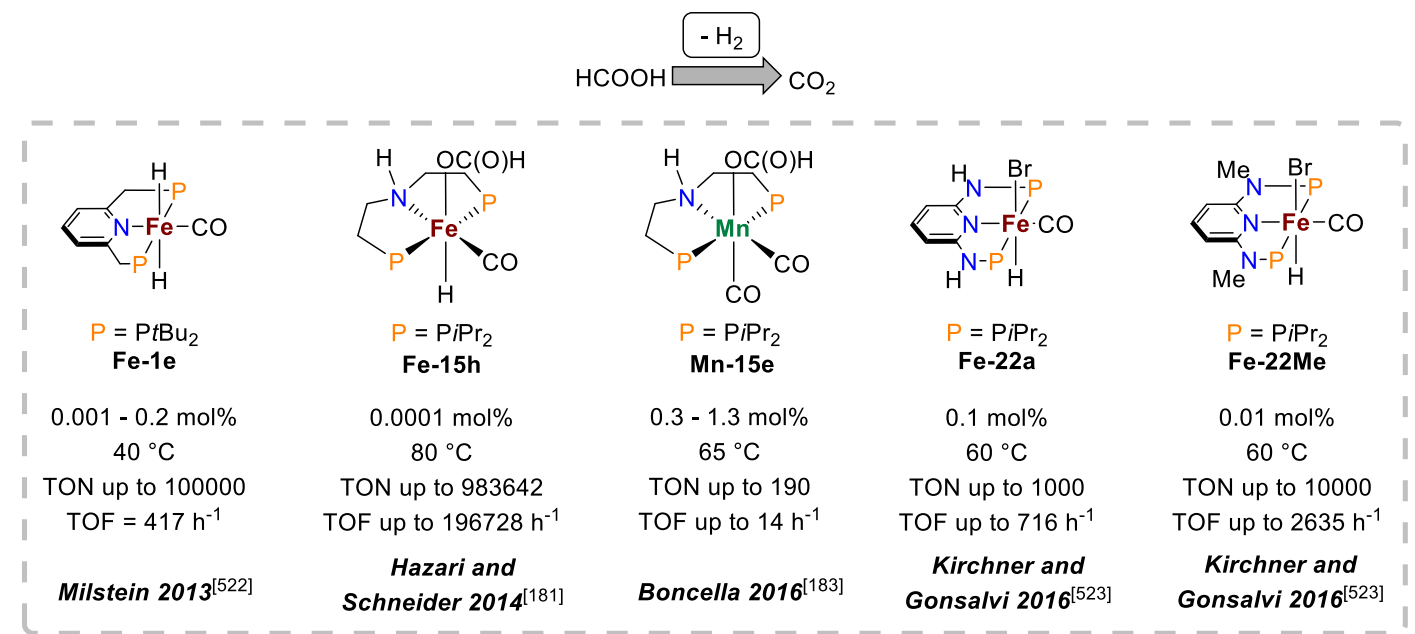

Figure 41. Summary of Formic Acid Hydrogenation Catalysts Based on PNP Pincer Ligands.

Boncella and coworkers reported FAD with an analogous manganese catalyst. ${ }^{183}$ With formate pincer complex $\left({ }^{i P r} \mathbf{P}\right.$ $\left.\mathbf{N}^{\mathrm{H}} \mathbf{P}\right) \mathrm{Mn}\left(\mathrm{O}_{2} \mathrm{CH}\right) \mathrm{H}(\mathrm{CO})(\mathbf{M n - 1 5 e})$ TON up to 190 were obtained with catalyst loadings of $0.3-1.3 \mathrm{~mol} \%$.

\subsection{Dehydrogenation of Nitrogen-Containing Substrates}

4.3.1. Amineboranes Ammonia borane $\left(\mathrm{H}_{3} \mathrm{NBH}_{3}, \mathrm{AB}\right)$ also qualifies as candidate for chemical hydrogen storage due to the high gravimetric hydrogen density. ${ }^{524,525}$ Furthermore, it can be used as precursor to polymers and materials (e.g. polyaminoborans, polyborazines, boron nitride) that represent inorganic analogues of polyhydrocarbons and carbonaceous materials. ${ }^{526}$ While thermal $\mathrm{AB}$ is generally possible, catalytic $\mathrm{AB}$ dehydrocoupling enables selectivity control.

Scheme 89. Catalytic dehydrogenation of ammonia borane catalyzed by ${ }^{i \mathrm{Pr}} \mathbf{F e}-\mathbf{1 5 a}\left(\mathrm{P}={\left.\mathrm{P} i \mathrm{Pr}_{2}\right)}^{527}\right.$
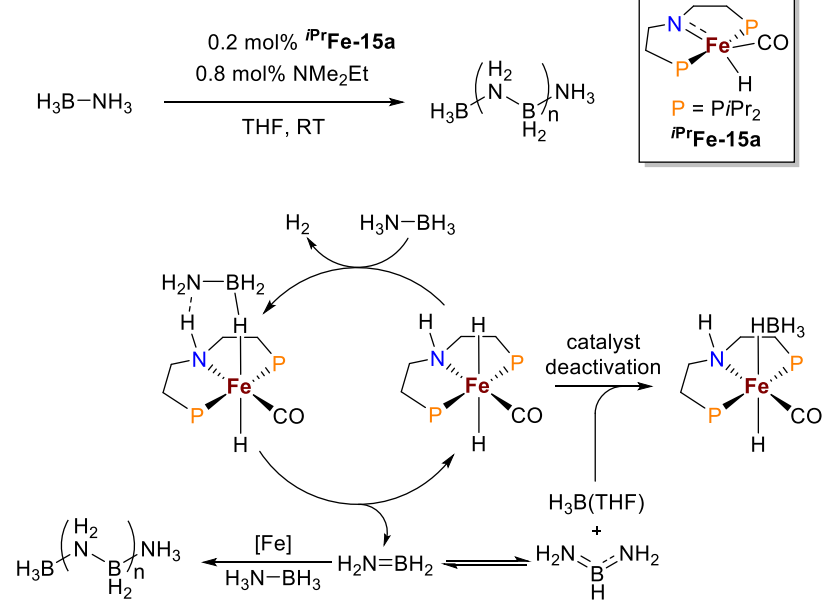

Holthausen, Schneider and co-workers demonstrated the catalytic dehydrogenation of $\mathrm{AB}$ to polyaminoborane and 1 equiv. $\mathrm{H}_{2}$ with low loadings $(0.2 \mathrm{~mol} \%)$ of the iron amido

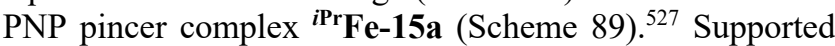

by computational treatment, the authors propose an alliron(II) MLC cycle with $\mathrm{H}_{2}$ 1,2-addition to the catalyst by $\mathrm{H}^{+} / \mathrm{H}^{-}$transfer from the $\mathrm{N}-$ and $\mathrm{B}$-termini of the substrate, respectively, in analogy to an earlier report on a related ruthenium catalyst. ${ }^{187}$

${ }^{11} \mathrm{~B}$ NMR spectroscopy indicated the formation of diaminoborane $\mathrm{HB}\left(\mathrm{NH}_{2}\right)_{2}$ as a result of free aminoborane dimutation, which also releases $\mathrm{BH}_{3}$ as a byproduct. Accordingly, the borohydride complex ${ }^{i \mathbf{P r}} \mathbf{F e - 1 5 g}$ was spectroscopically observed, which exhibits considerably lower catalytic activity than parent ${ }^{i \mathbf{P r}} \mathbf{F e - 1 5 a}$. This observation led to a threefold improvement of the TON for this and the previously reported ruthenium catalyst upon trapping free $\mathrm{BH}_{3}$ by addition of NEtMe2.

The group of Beweries later reported the dehydrogenation of methylamine borane with the less active borohydride com-

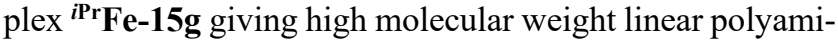
noborane at room temperature. ${ }^{528}$ Interestingly, off-metal polymerization of highly reactive dehydrogenation product aminoborane $\left(\mathrm{H}_{2} \mathrm{NBH}_{2}\right)$ was proposed despite evidence for a polymer chain growth mechanism.

In a mechanistic study, Guan and coworkers evaluated the iron POCOP pincer complex Fe-38 for catalytic AB dehydrogenation. ${ }^{529}$ An unusually high degree of $\mathrm{H}_{2}$ release (2.32.5 equiv. per $\mathrm{AB}$ ) was obtained.

The authors proposed an all-iron(II) catalytic cycle (Scheme 90) involving an unusual MLC mechanism of the aryl pincer ligand. As the key step protonolysis of the aryl group by the AB $N$-terminus was proposed leading to release of free aminoborane. Subsequent $\mathrm{H}_{2}$ elimination from a dihydride intermediate with a bidentate arene ligand restores the iron ipsoarene bond. 


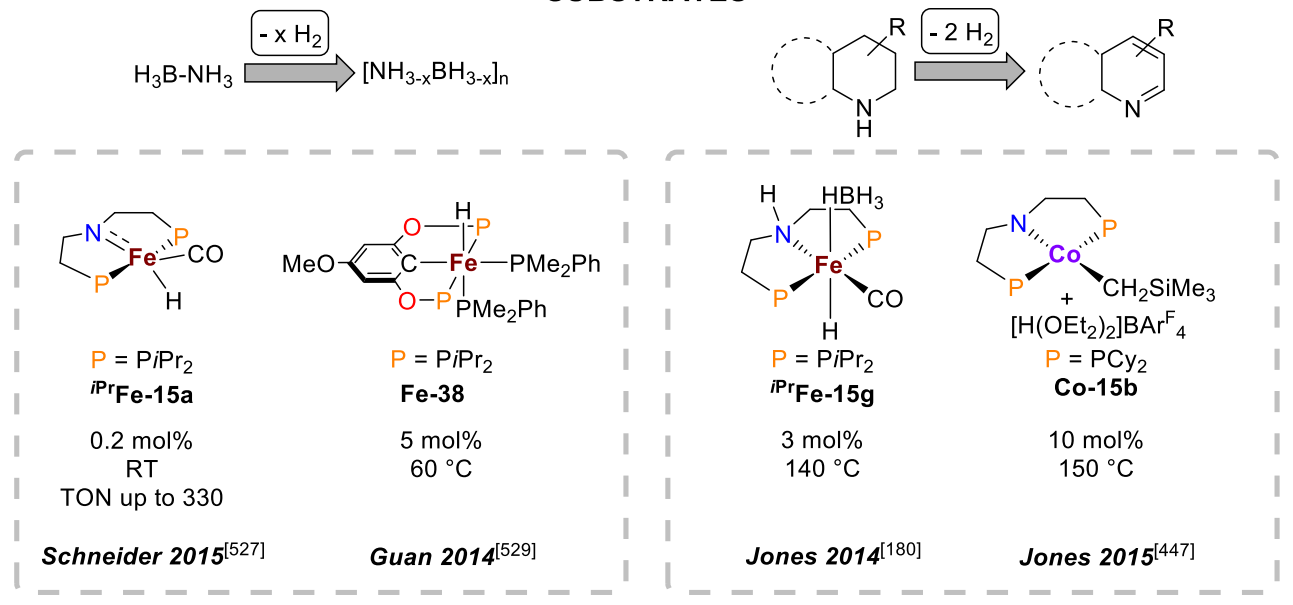

Figure 42. Summary of Dehydrogenation Catalysts for $N$-Containing Substrates Based on PNP and POCOP Pincer Ligands.

Scheme 90. Proposed catalytic cycle for AB dehydrogenation catalyzed by $\mathbf{F e - 3 8}\left(\mathrm{P}={\left.\mathrm{P} i \mathrm{Pr}_{2}\right)}^{529}\right.$

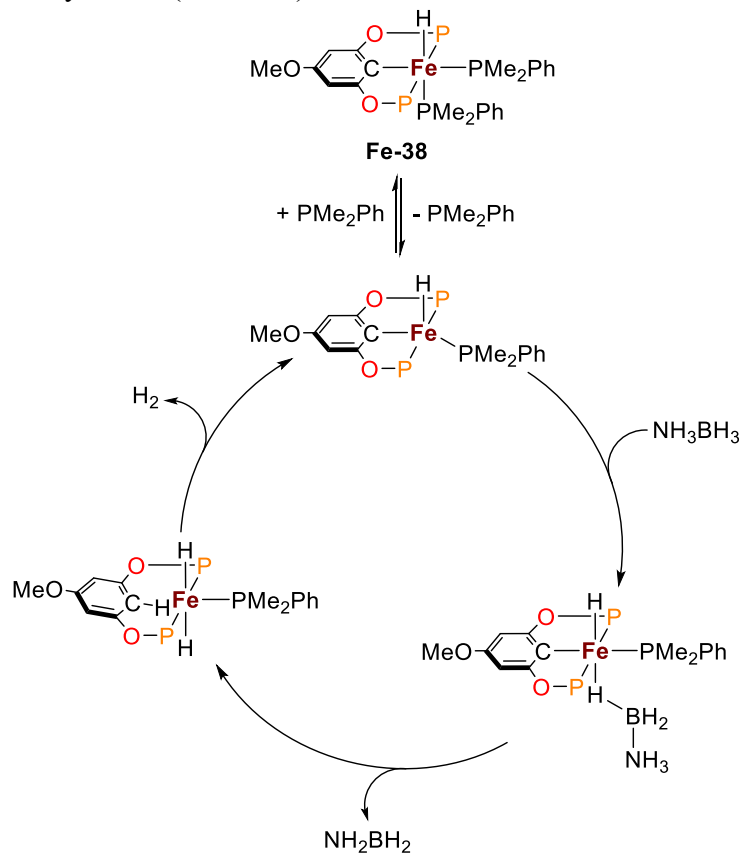

4.3.2. $\mathrm{N}$-Heterocycles Jones and coworkers reported the dehydrogenation of several $N$-heterocycles in moderate to excellent yields utilizing the borohydride complex ${ }^{i \mathbf{P r}} \mathbf{F e}-$ 15g as catalyst (Scheme 91). ${ }^{180}$ A MLC catalytic cycle was proposed with in situ formed dihydride $\left({ }^{i{ }^{2}} \mathbf{P N}^{\mathrm{H}} \mathbf{P}\right) \mathrm{Fe}(\mathrm{H})_{2} \mathrm{CO}$ and amido ${ }^{i P r} \mathbf{F e}-15 \mathbf{a}$ as active species. No semidehydrogenated intermediate was observed.
Scheme 91. Dehydrogenation of $N$-heterocycles with precatalyst complex ${ }^{i \text { Pr }} \mathbf{F e}-\mathbf{1 5 g}$. ${ }^{180}$

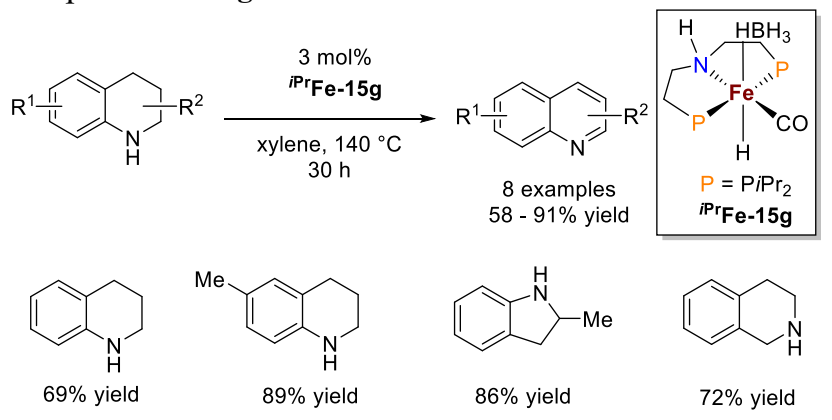

Subsequently, Hanson's catalyst $\left(\mathbf{C o - 1 5 a} /\left[\mathrm{H}\left(\mathrm{OEt}_{2}\right)_{2}\right] \mathrm{BAr}_{4}^{\mathrm{F}}\right.$; see Section 3.1.2) was used for the dehydrogenation of $N$ heterocycles (Scheme 92 ). ${ }^{447}$ With $10 \mathrm{~mol} \%$ catalyst loading five nitrogen containing heterocycles were dehydrogenated in predominantly excellent yields. Only substrates with annulated phenylene rings could be oxidized. Interestingly, the $\mathrm{N}$-methylated cobalt complex is inactive in dehydrogenation but catalyzes the reverse hydrogenation reaction suggesting different mechanisms for forward and reverse direction.

Scheme 92. Dehydrogenation of $N$-Heterocycles with Hanson's catalyst $(\mathbf{C o - 1 5 b}){ }^{447}$

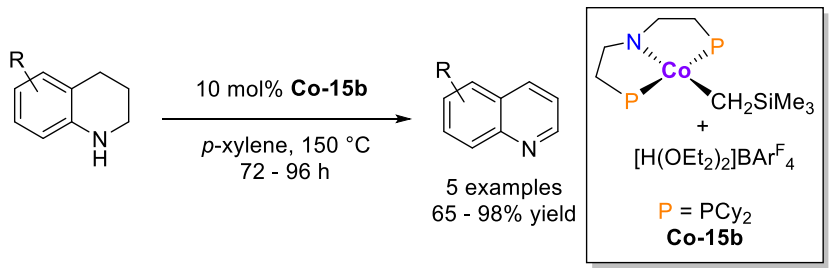

4.3.3. Dehydrogenative $\mathrm{C}-\mathrm{N}$ Coupling The first dehydrogenative synthesis of lactams catalyzed by iron pincer complexes was reported by Beller and coworkers (Scheme 93). ${ }^{510}$ Precatalyst complex ${ }^{i \mathbf{P r}} \mathbf{F e}-\mathbf{1 5 g}$ was used to transform nine aminoalcohols into the respective lactams. Despite higher catalyst loading yields are generally lower than for lactone synthesis. Four-membered $\beta$-lactams, which 
lead structures in antibiotic drugs, could not be obtained with this methodology. ${ }^{530-532}$ In contrast, five- to eight-membered heterocycles were obtained in good yields. With annelated phenylene groups indoles and 3,4-dihydroisoquinolines were obtained, due to competing dehydration of the hemiaminal intermediates.

Scheme 93. Dehydrogenative coupling of aminoalcohols to lactams catalyzed by ${ }^{i P r} \mathbf{F e}-15 g$ (a: ${ }^{1} \mathrm{H}$ NMR spectroscopic yield $)^{510}$
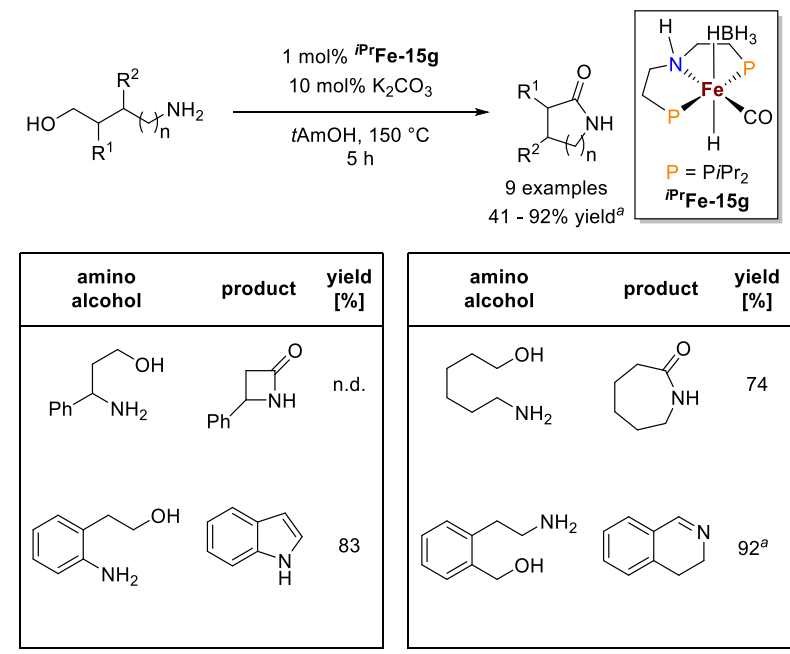

Bernskoetter and coworkers reported highly efficient intermolecular dehydrogenative coupling of alcohols and secondary amines to (form)amides using low loadings (0.1 mol\%) of iron amide complex ${ }^{i \mathrm{Pr}} \mathbf{F e}-\mathbf{1 5 a} .{ }^{533}$ A strong dependency on substrate sterics was observed, resulting in higher turnover numbers for sterically undemanding amines and alcohols (Scheme 94).

Scheme 94. Coupling of methanol and 1,2,3,4-tetrahydroisoquinoline catalyzed by ${ }^{i \text { Pr Fe-15a. }}{ }^{533}$

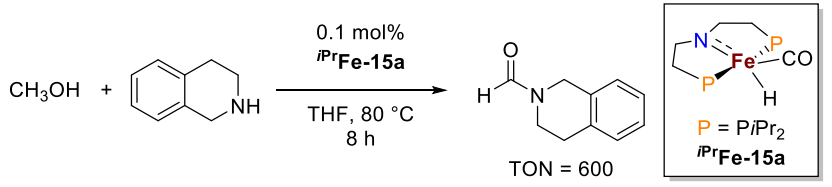

Milstein and coworkers reported dehydrogenative $\mathrm{N}$ formylation of amines using methanol as $\mathrm{C}_{1}$ building block catalyzed by the PNP manganese pincer complex Mn-28a (Scheme 95). ${ }^{534}$ This process represents the reverse reaction of the last step within $\mathrm{CO}_{2}$ to methanol hydrogenation via formamide intermediates established by Prakash and coworkers, as discussed in Section 3.6. ${ }^{480}$ A range of 14 aromatic and aliphatic amines could be $N$-formylated in moderate to good yields at $110^{\circ} \mathrm{C}$ in methanolic solution.
Scheme 95. Dehydrogenation reactions catalyzed by Mn-28a. ${ }^{534}$ 536

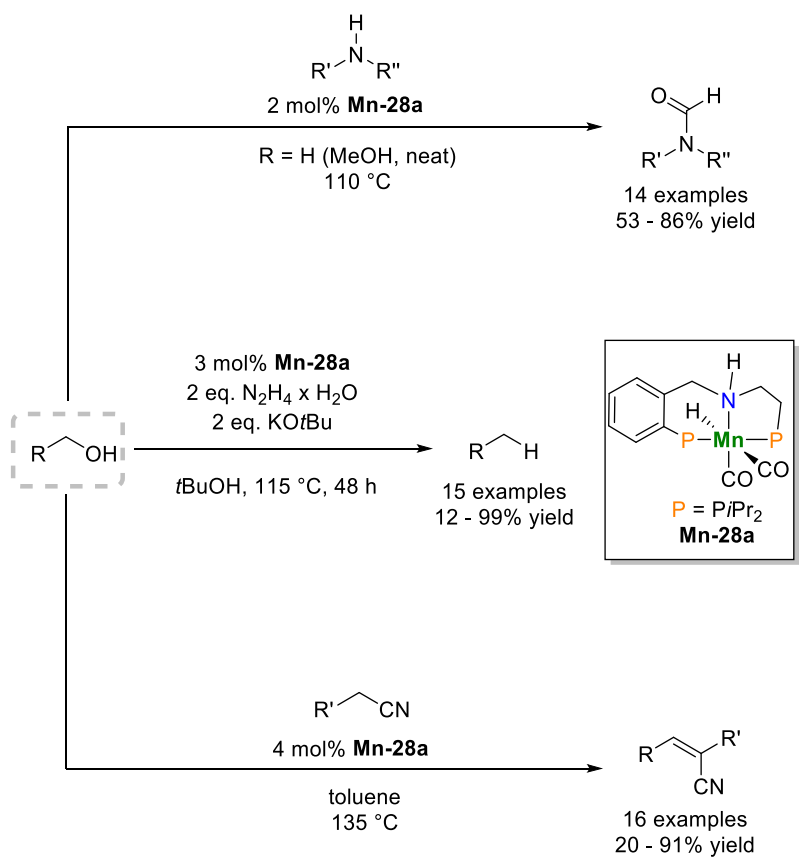

Stoichiometric experiments suggest an unusual variant of MLC with this ligand. The manganese dicarbonyl amide complex Mn-28c was isolated from dehydrohalogenation of bromide Mn-28b (Scheme 96) and adds methanol by 1,2addition across the $\mathrm{Mn}-\mathrm{N}$ bond. These observations suggest the typical all-Mn(I) amide / hydride amine cycle for alcohol dehydrogenation. However, the amide complex Mn-28c turned out to be thermally unstable and isomerizes under catalytic conditions $\left(110^{\circ} \mathrm{C}\right)$ to the benzylic $\mathrm{C}-\mathrm{H}$ metalated amine tautomer Mn-28d (Scheme 96). In fact, $\mathrm{H}_{2}$ elimination from the hydride amine catalyst Mn-28a directly leads to Mn-28d, which was computed to be more stable than the amide isomer by $9.7 \mathrm{kcal} \cdot \mathrm{mol}^{-1}$. Importantly, $\mathbf{M n - 2 8 d}$ is catalytically active and therefore represents the catalyst resting state. Formation of the final product is maintained by subsequent dehydrogenation of the hemiaminal intermediate that results from addition of amine to formaldehyde. 
DEHYDROGENATIVE C-N COUPLING

AND AMINOMETHYLATION

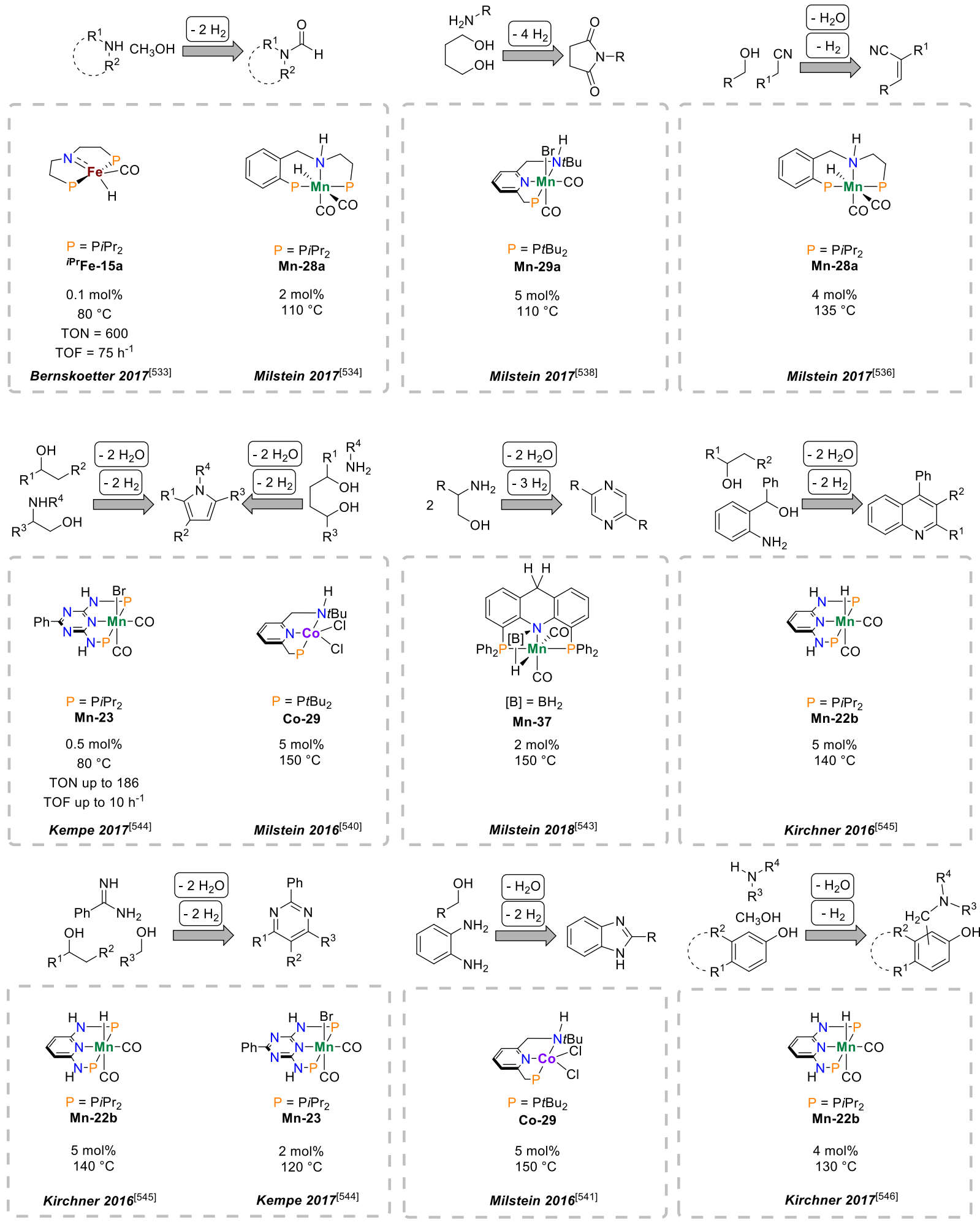

Figure 43. Summary of catalysts for the dehydrogenative C-N coupling and aminomethylation based on PNP and PNN pincer ligands.

Milstein subsequently established cascade-type alcohol functionalization that is based on initial manganese catalyzed dehydrogenation. As a first example, catalyst Mn28a was further utilized in the deoxygenation of primary 
alcohols (Scheme 95). ${ }^{535}$ With hydrazine as reductant, a range of 15 primary alcohols transformed to the respective alkanes in good yields. Other reducible or coordinating functional groups led to significantly lower yields, e.g. for the allylic alcohol (E)-3-phenylprop-2-en-1-ol giving $53 \% 1$ phenylpropane. The authors propose that the product is formed by Wolff-Kishner reaction of the aldehyde from manganese catalyzed alcohol dehydrogenation via an MLC mechanism. In the absence of base deoxygenation is also observed, yet with lower yields, suggesting that Mn catalyzed hydrazone N-H activation might be possible.

Scheme 96. Stoichiometric reactivity of the manganese pincer platform $\mathrm{Mn-28b}\left(\mathrm{P}={\left.\mathrm{P} i \mathrm{Pr}_{2}\right)}^{534}\right.$

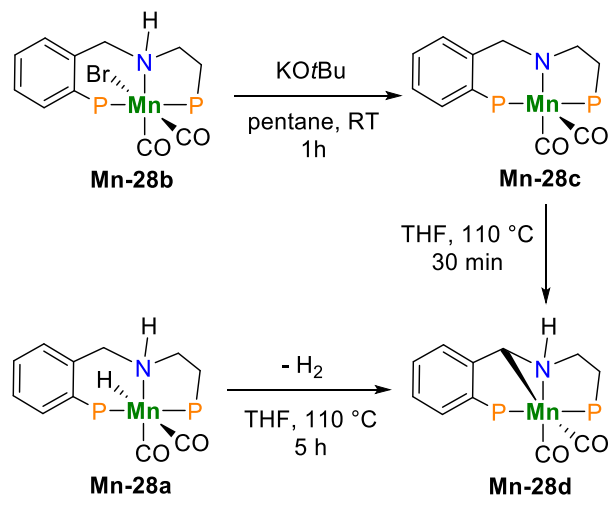

Dehydrogenative $\alpha$-olefination of nitriles with primary alcohols as alkylidene source was subsequently reported for catalyst Mn-28a (Scheme 95). ${ }^{536}$ A range of 16 different vinyl nitriles were obtained in low to very good yields using 4 mol\% Mn-28a. Substrate scope includes (hetero)aromatic as well as aliphatic primary alcohols and predominantly benzylic nitriles. As for nitrile deoxygenation, the authors propose cascade-type functionalization of the in situ formed aldehyde from primary AAD, in this case by Knoevenagel condensation with the nitrile. Besides AAD, the catalyst was ascribed an active role in nitrile activation, which are more acidic $\left(\mathrm{p} K_{\mathrm{a}}^{\mathrm{dmso}}\left(\mathrm{PhCH}_{2} \mathrm{CN}\right)=22\right)^{537}$ than primary alcohols (Scheme 97). It remains unclear if free or coordinated cyanophenylmethanide undergoes the $\mathrm{C}-\mathrm{C}$ bond forming step.

The pyridine-based PNN pincer catalyst Mn-29a was used to synthesize cyclic imides via acceptorless dehydrogenative coupling of diols and amines releasing 4 equiv. $\mathrm{H}_{2}$ (Scheme 98). ${ }^{538}$ With $5 \mathrm{~mol} \%$ catalyst loading and activation with 10 mol\% KH, a range of 16 cyclic imides were obtained. Benzylic amines generally give higher yields than aliphatic amines. In the absence of the amine, the respective lactones are obtained, which give imides with the amine under catalytic conditions and might therefore be intermediates. In a related study the same catalyst was used for linear amide synthesis by dehydrogenative coupling of alcohols and amines. ${ }^{539}$
Scheme 97. Proposed mechanism for $\alpha$-olefination of nitriles catalyzed by $\mathbf{M n - 2 8 a}\left(\mathrm{P}={\left.\mathrm{P} i \mathrm{Pr}_{2}\right) .}^{536}\right.$

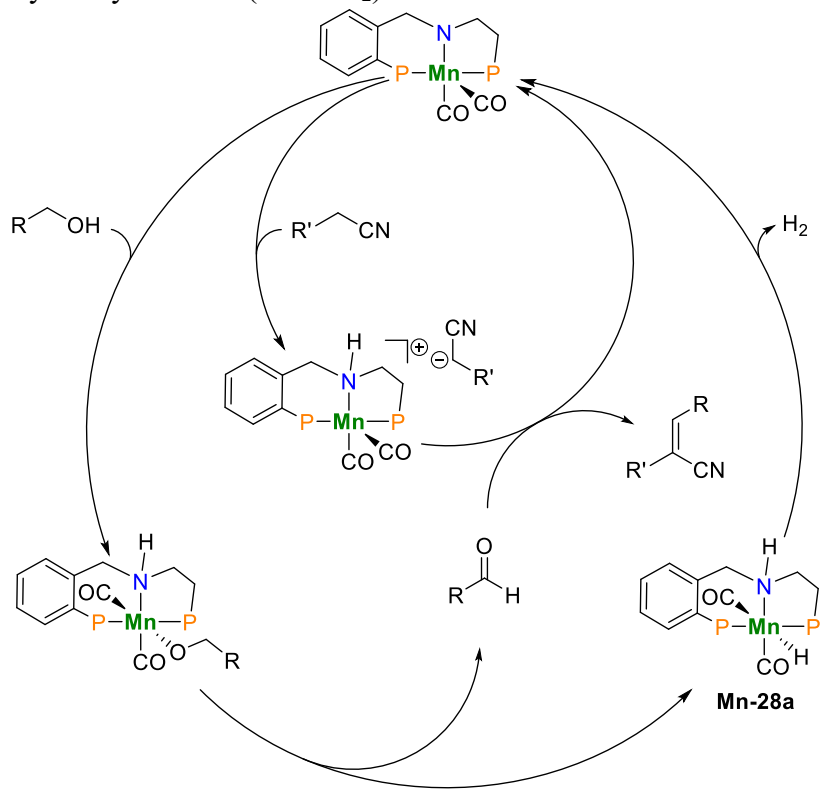

The proposed mechanism implies stepwise, MLC-assisted, catalytic dehydrogenation to lactone and subsequent aminolysis or alternatively hemiaminal formation on the intermediate aldehyde stage. MLC is supported by the catalytic inactivity of the analogous complex with tertiary amine $\mathrm{CH}_{2} \mathrm{NEt}_{2}$ pincer arm. Furthermore, the amide derivative of $\mathbf{M n - 2 9 a}$ is also catalytically active. Hemilability of the amine donor was discussed as being potentially useful for $\beta$ - $\mathrm{H}$ elimination at the alkoxide intermediate.

Scheme 98. Dehydrogenative lactam synthesis from diols and amines catalyzed by $\mathbf{M n}-\mathbf{2 9 a}$. $^{538}$

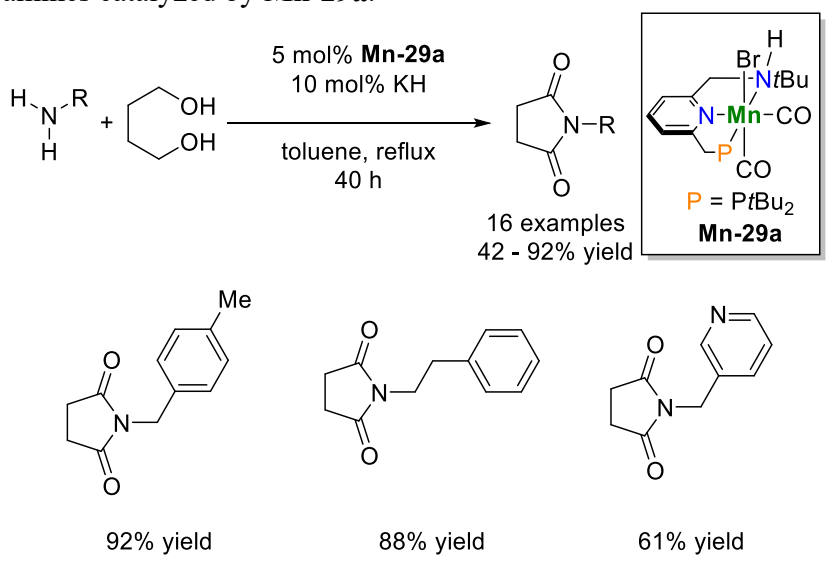

This approach has lead to several protocols for the synthesis of aromatic heterocycles. Dehydrogenation of secondary 1,4-diols by the cobalt pincer precatalyst $\mathbf{C o - 2 9}(5 \mathrm{~mol} \%)$ in the presence of primary amines provides a versatile route to a range of 1,2,5-substituted pyrroles in low to excellent yields (Scheme 99). ${ }^{540}$ Catalytic amounts of a hydride source $\left(\mathrm{NaBEt}_{3} \mathrm{H}\right)$ and base are further required. Stoichiometric reaction with $\mathrm{NaBEt}_{3} \mathrm{H}$ produced a cobalt(I) chloride complex that exhibits similar catalytic activity ${ }^{467}$ This cobalt platform is also an active catalyst for the synthesis of benzimidazoles by dehydrogenative coupling of aromatic diamines 
and primary alcohols (Scheme 99). ${ }^{541}$ Under base-free conditions a range of 18 benzimidazoles was isolated.

Scheme 99. Dehydrogenative cobalt-catalyzed coupling of diols with primary amines to form substituted pyrroles. ${ }^{540,541}$

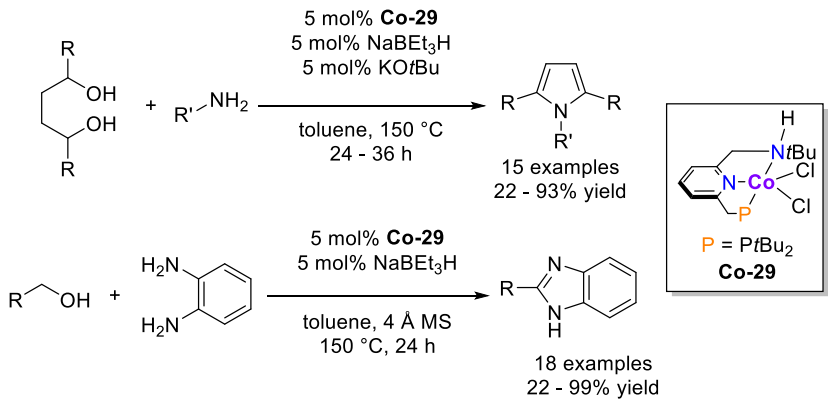

Balaraman and coworkers reported the use of a cobalt(II) SNS aminopincer precatalyst for the dehydrogenative coupling of aminoalcohols and alcohols to substituted $N$-heterocycles. ${ }^{542}$ A broad range of 15 pyrroles, 11 pyridines and quinolines and one pyrazine was obtained in moderate to good yields.

Pyrazines and quinoxalines were synthesized by Milstein with the dehydrogenative coupling approach catalyzed by the acridine-based manganese pincer complex Mn-37 (Scheme 100). ${ }^{543}$ Activation was achieved using potassium hydride at high temperatures in toluene, releasing dihydrogen and water as stoichiometric byproducts.

Scheme 100. Manganese catalyzed synthesis of pyrazines and quinoxalines via dehydrogenative coupling. ${ }^{543}$

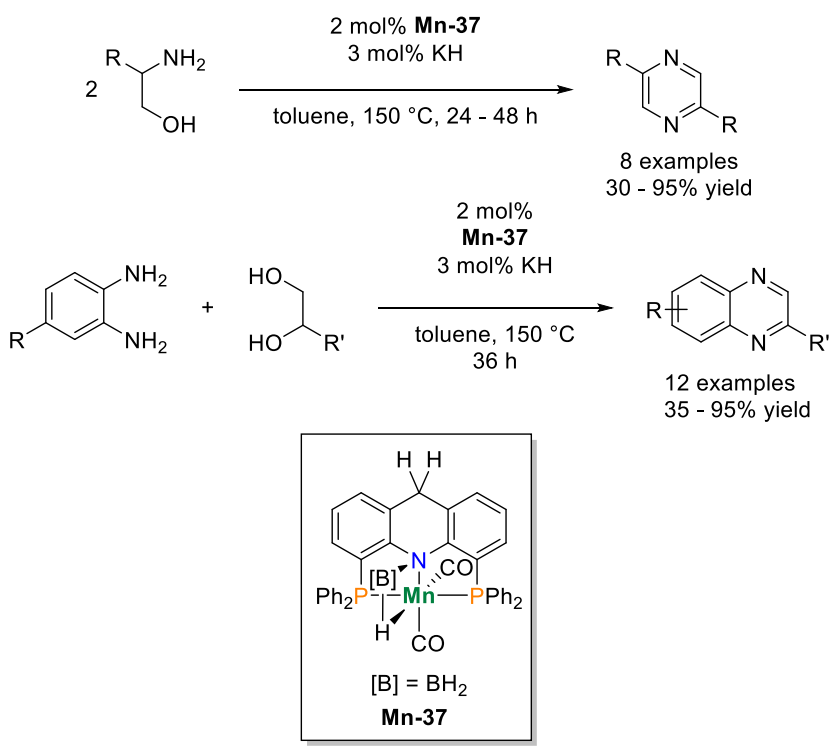

Manganese catalysts based on the $\mathbf{P N}_{\mathbf{3}} \mathbf{P}$ and $\mathbf{P N}_{5} \mathbf{P}$ pincer ligand system are also used for dehydrogenative multicomponent syntheses of nitrogen containing heterocycles like pyrroles, quinolines and pyrimidines. Kempe and coworkers demonstrated the formation of 2,3,5-substituted pyrroles via dehydrogenative coupling of secondary alcohols with aminoalcohols catalyzed by $\mathrm{PN}_{5} \mathrm{P}$ pincer ligated complex Mn23 (Scheme 101). ${ }^{544} \mathrm{~A}$ broad range of 20 products and 9 further examples with a similar precatalyst could be obtained. Iron and cobalt complexes with the same type of pincer ligands showed no catalytic activity and the manganese catalyst even surpasses the yields obtained with an analogous iridium complex at milder reaction conditions. Stoichiometric reaction of $\mathbf{M n - 2 3}$ with $\mathrm{KO} t \mathrm{Bu} / \mathrm{H}_{2}$ gave the respective manganese(I) hydride, which is also catalytically active, leading to a proposed all-manganese(I) dearomatized amide / aromatized hydride cycle.

Kirchner and coworkers utilized the related aromatized $\mathrm{PN}_{3} \mathrm{P}$ hydride catalyst $\mathbf{M n - 2 2 b}$ for quinoline synthesis upon dehydrogenative coupling of 2-aminobenzyl alcohol with secondary alcohol (Scheme 101, bottom arrow) ${ }^{545}$ Interestingly, the $N$-methylated analogon of $\mathbf{M n - 2 2 b}$ proved inactive, suggesting the relevance of MLC. A range of 16 quinolines was synthesized with a catalyst loading of $5 \mathrm{~mol} \%$ in the presence of $\mathrm{KO} t \mathrm{Bu} / \mathrm{KOH}$ at $140{ }^{\circ} \mathrm{C}$.

Scheme 101. Dehydrogenative multicomponent reactions to pyrroles, quinolines and pyrimidines catalyzed by manganese $\mathbf{P N}_{\mathbf{3}} \mathbf{P}$ and $\mathbf{P N} \mathbf{N}_{5} \mathbf{P}$ pincer complexes. ${ }^{250,544,545}$

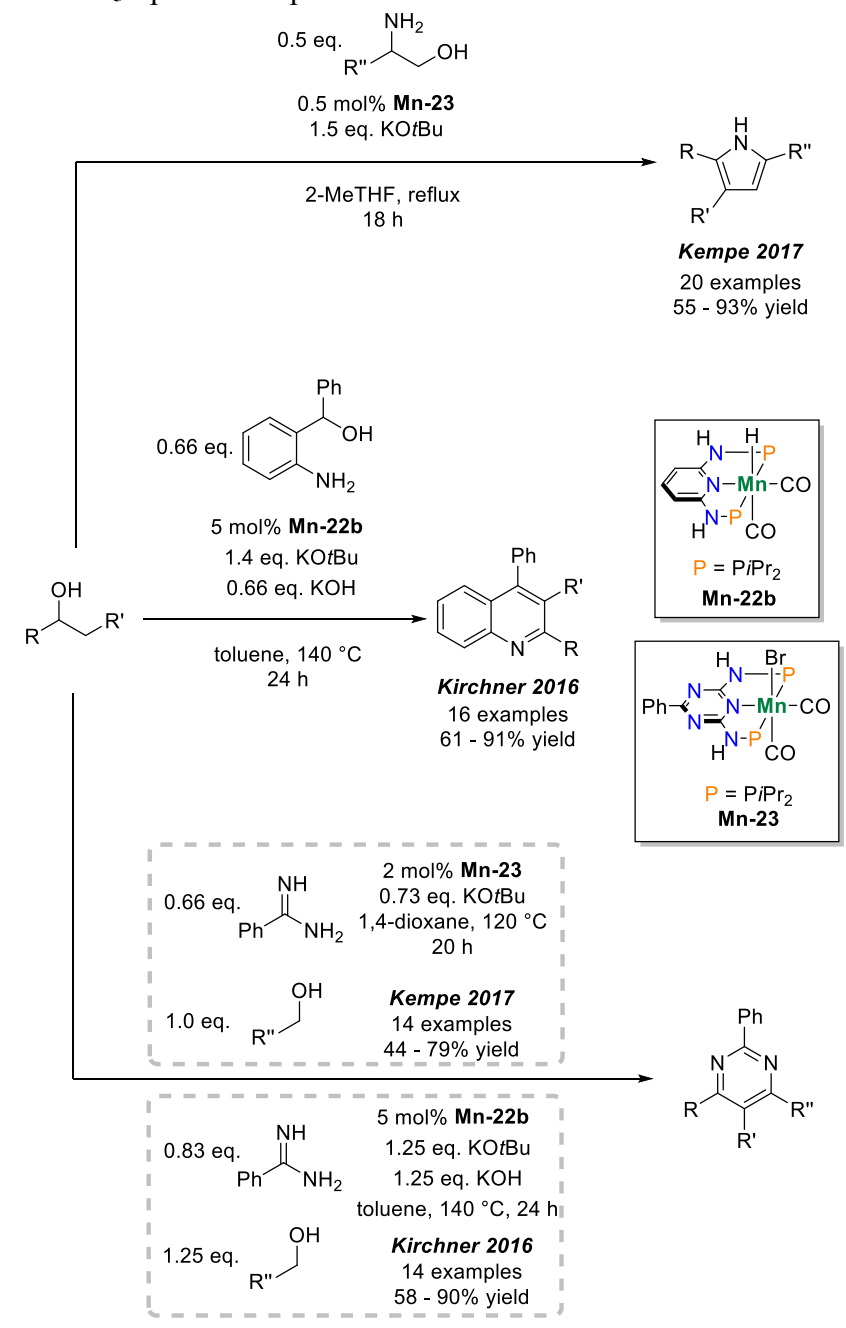

Both manganese platforms were utilized for dehydrogenative coupling of benzamidine with primary and secondary alcohols to substituted pyrimidines (Scheme 101). With Kirchner's catalyst $\mathbf{M n - 2 2 b}$, addition of $\mathrm{KOH}$ is required, ${ }^{545}$ 
while Kempe's catalyst Mn-23 operates at lower temperatures only using $\mathrm{KO} t \mathrm{Bu}$ as base. ${ }^{250}$

\subsubsection{Dehydrogenative Aminomethylation De-} hydrogenative aminomethylation of aromatic compounds with methanol as the $\mathrm{C}_{1}$ building block has been reported by Kirchner and co-workers using the monohydride catalyst Mn-22b (Scheme 102). ${ }^{546}$ A range of 28 (hetero)aromatic products were obtained in moderate to good yields with a catalyst loading of $4 \mathrm{~mol} \%$ and 1.3 equivalents $\mathrm{KO} t \mathrm{Bu}$. In the absence of amine two aromatic cores were coupled to bisarylmethane products. With the related iron(II) hydride complex Fe-22a, methylation aromatics were mainly obtained (ca. 50\%).

Scheme 102. Dehydrogenative aminomethylation of aromatic compounds catalyzed by manganese(I) catalyst Mn-22b. ${ }^{546}$

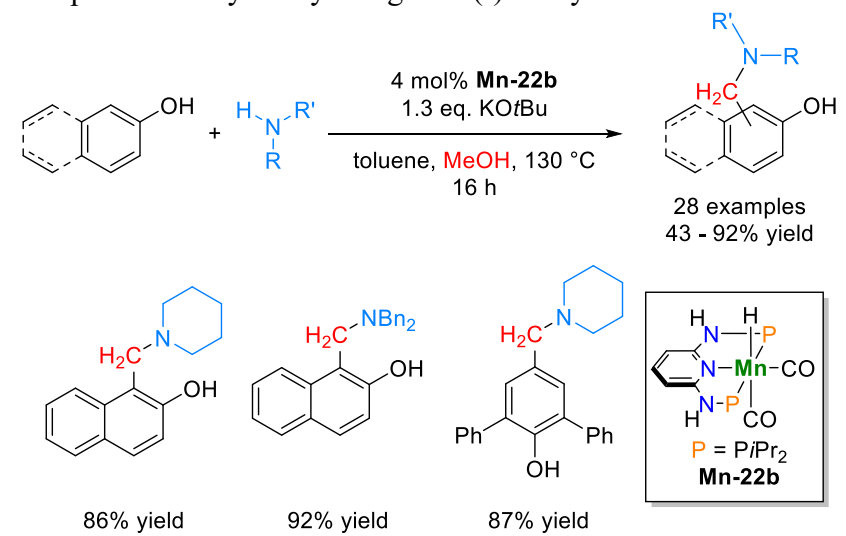

\section{HYDROGEN METHODOLOGY}

BORROWING

The 'hydrogen borrowing' methodology is a synthetic strategy for transformations via dehydrogenation, functionalization and rehydrogenation reactions that are net redox-neutral. $^{547-553}$ The dehydrogenation of the nucleophilic alcohol group implies an umpolung to the electrophilic carbonyl functionality thereby opening up follow-up reactivity, like condensation reactions or aldol reactivity. Typical reactions are condensations like $\mathrm{N}$-alkylation reactions, carbonyl and alcohol -alkylations (Scheme 103) that allow for versatile $\mathrm{C}-\mathrm{N}$ and $\mathrm{C}-\mathrm{C}$ bond formation with alcohols as hydrocarbon source. The hydrogen borrowing methodology is therefore an attractive approach for expanding the scope of renewable carbon sources as feedstock for value-added products.

Hanson and coworkers reported the base-free synthesis of imines by AAD with cobalt(II) catalyst ( $\left.{ }^{\mathrm{Cy}} \mathbf{P N P}\right) \mathrm{Co}\left(\mathrm{CH}_{2} \mathrm{SiMe}_{3}\right) /\left[\mathrm{H}\left(\mathrm{OEt}_{2}\right)_{2}\right] \mathrm{BAr}_{4}{ }_{4}(\mathbf{C o}-15 b)$ in the presence of primary amines (Scheme 103). ${ }^{554}$ This work was expanded by Zheng's group demonstrating that the addition of molecular sieves and longer reaction times lead to secondary amines in moderate to excellent yields (Scheme 103). ${ }^{555}$ Under these conditions, the immediate imine condensation products are rehydrogenated to give a redox-neutral formal condensation reaction of the amine and alcohol precursors. Secondary amines could also be obtained with Hanson's catalyst from condensation of two amine precursors and elimination of ammonia (Scheme 103). ${ }^{556}$ Heterocoupling was achieved with primary or secondary amines and anilines to alkyl aryl amines. Diamines gave cyclic products.

Scheme 103. Hydrogen borrowing coupling reactions catalyzed by Hanson's catalyst. ${ }^{54-557}$
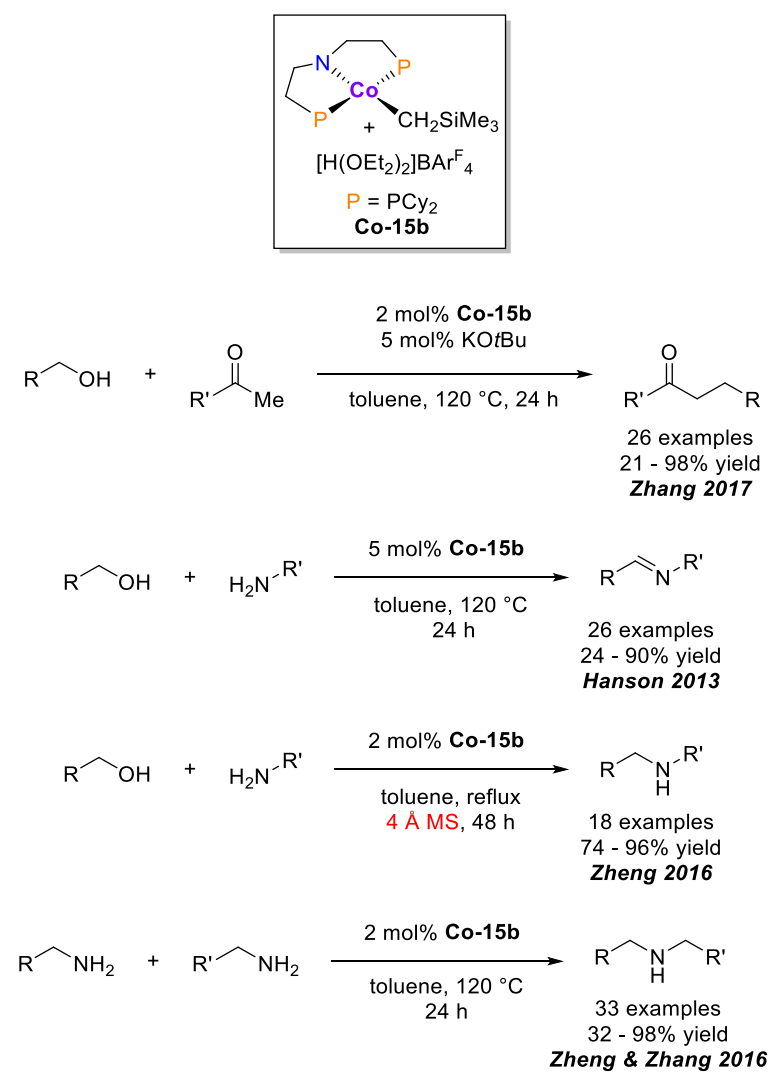

Further hydrogen borrowing applications with this catalyst were described, such as the $\alpha$-alkylation of ketones with primary alcohols (Scheme 103). ${ }^{557}$ This reaction relies on aldol condensation of the AAD product and subsequent catalytic $\mathrm{C}-\mathrm{C}$ rehydrogenation of the $\alpha, \beta$-unsaturated product (Scheme 103). In addition, a modified dehydrogenative Friedländer synthesis of quinolones was described with moderate yields that utilizes aminoalcohols and ketones, as discussed in Section 4.3.3.

Similar reactivity was reported by Kempe's group with their $\mathbf{P N}_{5} \mathbf{P}$ cobalt(II) precatalyst Co-23c. Dehydrogenative alkylation of aromatic amines with primary alcohols lead to a broad range of 33 secondary anilines and dianilines in moderate to excellent yields at low catalyst loading ( $2 \mathrm{~mol} \%$; Scheme 104). ${ }^{558}$ Related PN5 $\mathbf{P}$ catalyst Co-23b (5 mol\%) was used for alkylation of secondary alcohols with primary alcohols (Scheme 104). ${ }^{559}$ Stoichiometric experiments support the hydrogen borrowing mechanism. Catalysts Co-23b and $\mathbf{C o - 2 3 c}$ were further utilized for the $\alpha$-alkylation of unactivated amides and esters (Scheme 104). ${ }^{560} \mathrm{~A}$ broad range of $22 C$-alkylated amides and $12 C$-alkylated ester were obtained in moderate to high yields. The authors showed that 


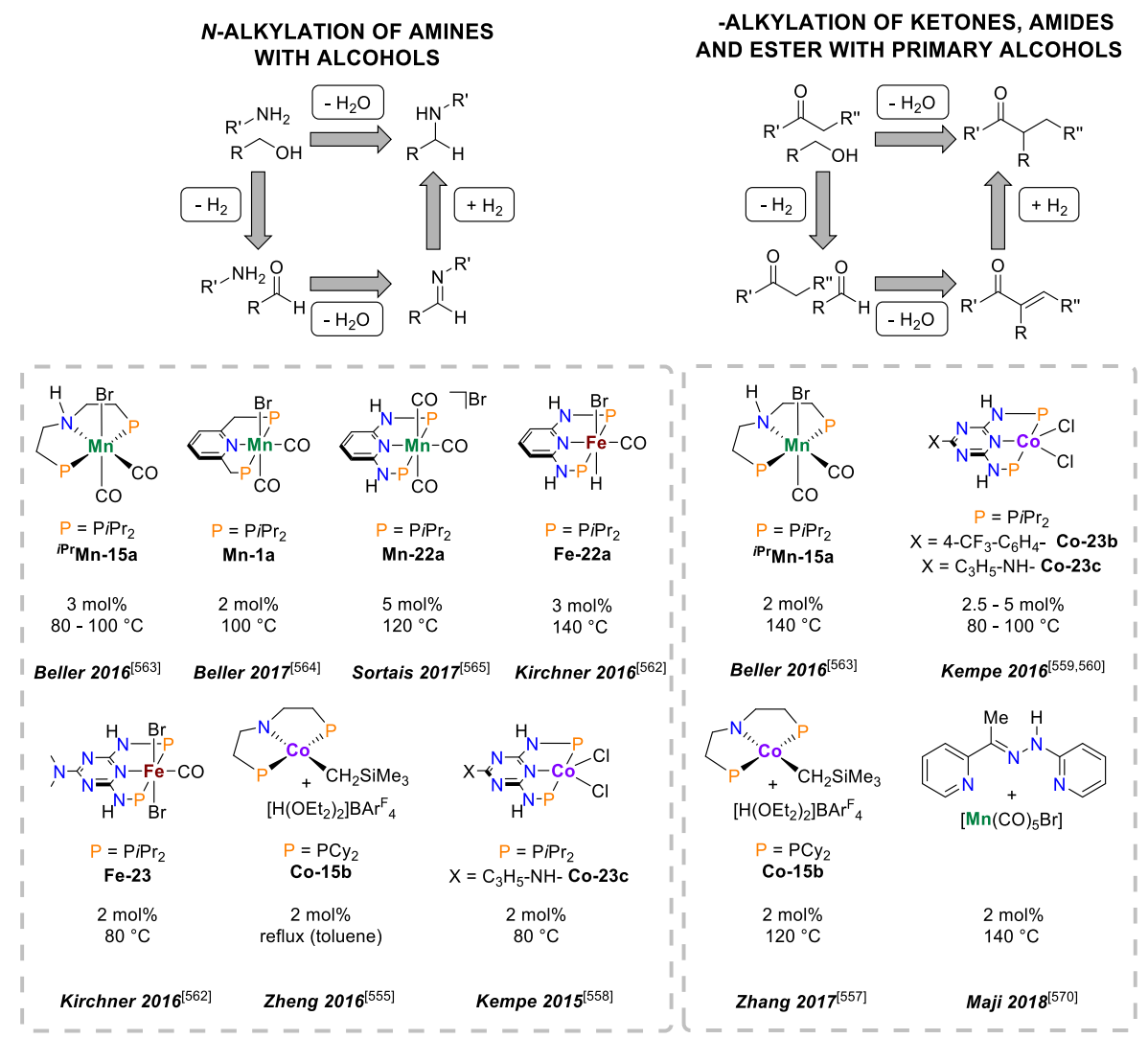

Figure 44. Summary of catalysts for the $N$-alkylation of amines with alcohols and for the coupling of amines involving a hydrogen borrowing reaction sequence based on PNP pincer ligands.

the steady-state concentration of the aldol condensation intermediate should be low to avoid side reactions.

Scheme 104. Hydrogen borrowing alkylations of amines, alcohols, amides and esters using cobalt $\mathbf{P N} \mathbf{N}_{5} \mathbf{P}$ catalysts. ${ }^{558-560}$

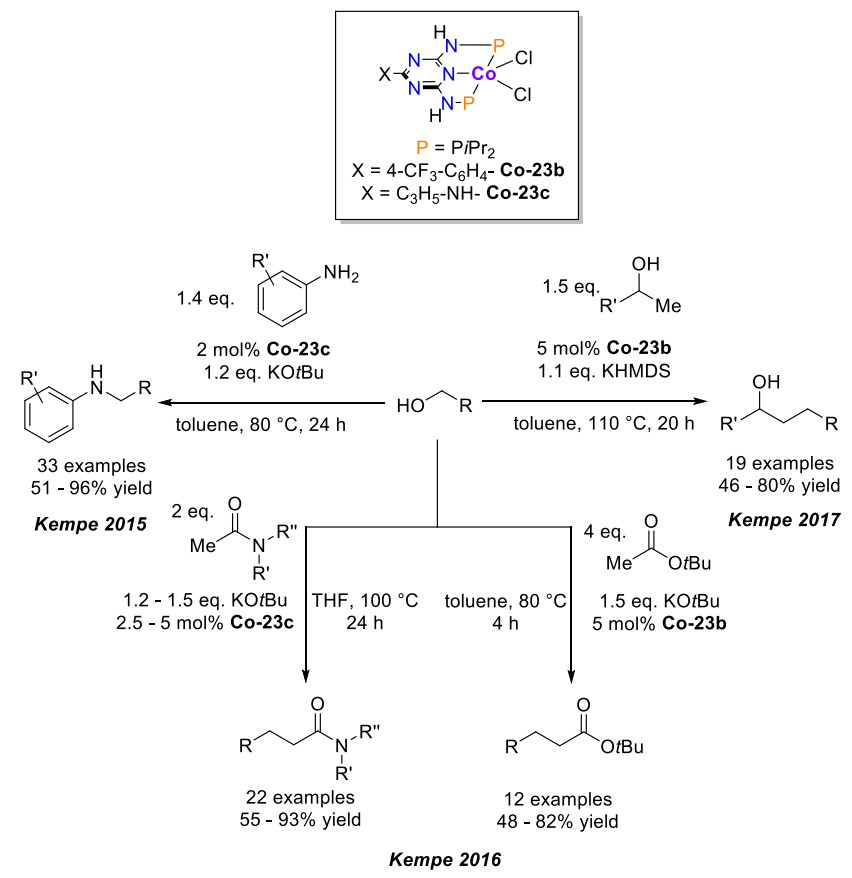

Intermolecular coupling of amines and alcohols was described by Kirchner and coworkers using iron(II) and manganese(I) pincer precatalysts with divergent reactivity. ${ }^{561}$ The Mn-based $\mathbf{P N} \mathbf{N}_{\mathbf{3}} \mathbf{P}$ catalysts selectively gave imines while the iron analogs resulted in the amine hydrogen borrowing products.

Scheme 105. Amine alkylation catalyzed by $\mathbf{P N}_{\mathbf{5}} \mathbf{P}$ iron catalyst Fe-23. ${ }^{562}$

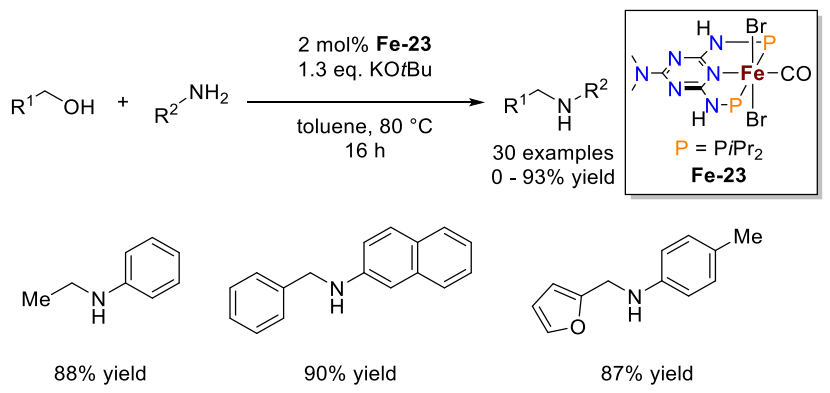

In a comparative study, the Kirchner group investigated a range of different $\mathbf{P N} \mathbf{N}^{\mathbf{P y}} \mathbf{P}, \mathbf{P N}_{\mathbf{3}} \mathbf{P}$ and $\mathbf{P N}_{5} \mathbf{P}$ iron pincer catalysts for amine alkyation (Scheme 105) ${ }^{562} \mathrm{~A}$ vast range of aliphatic and (hetero)aromatic amines was obtained in predominantly moderate to excellent yields with the $\mathrm{PN}_{5} \mathrm{P}$ catalyst Fe-23. A hydrogen borrowing mechanism via AAD, imine condensation and rehydrogenation with dearomatization/aromatization based MLC was proposed. 

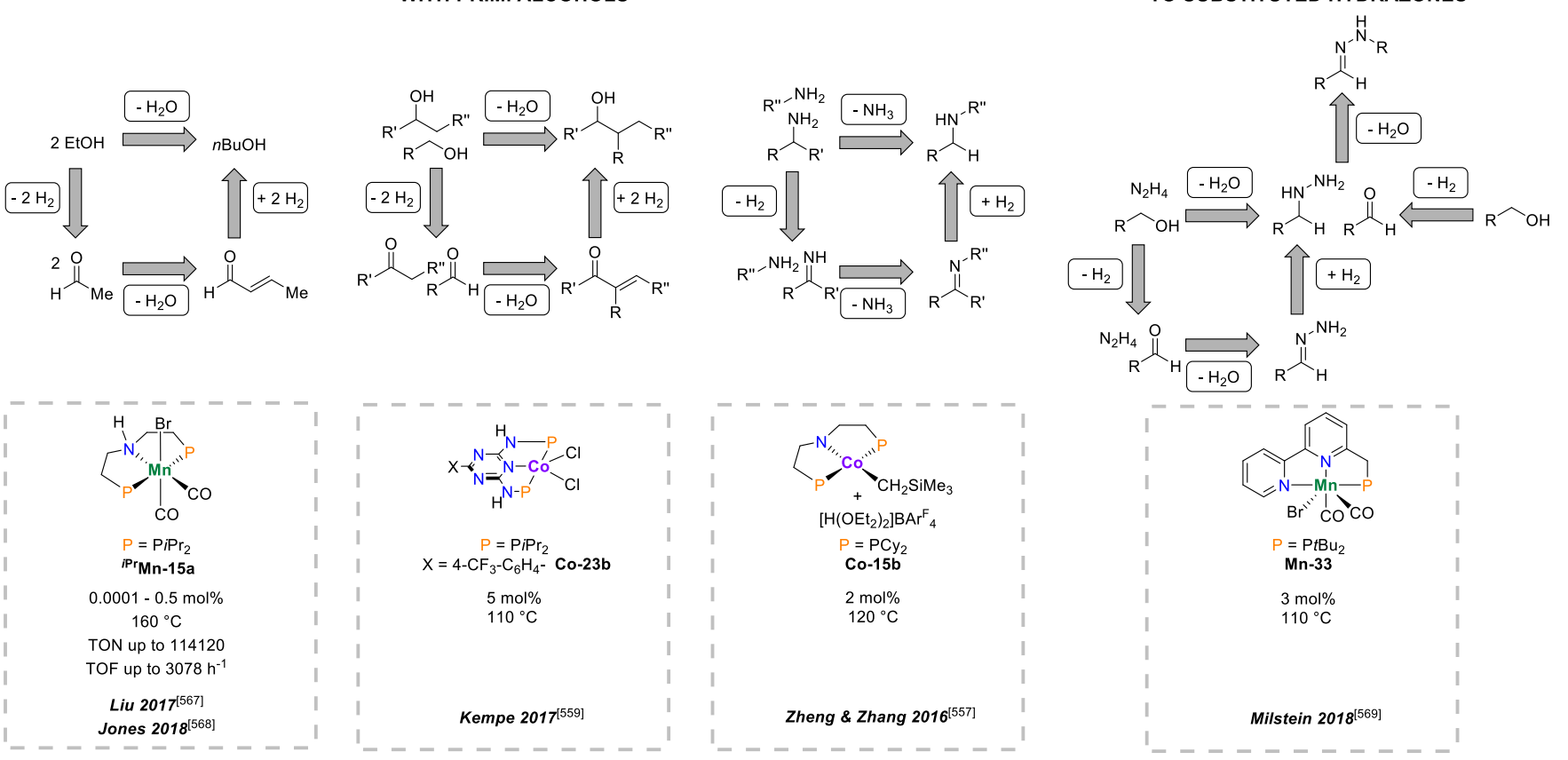

Figure 45. Summary of PNP and PNN pincer complexes catalyzing hydrogen borrowing reaction sequence incorporating transformations.

The Beller group introduced manganese pincer complexes to amine $N$-alkylation with alcohols. The diaminophosphinebased precatalyst ${ }^{i \operatorname{Pr}} \mathbf{M n}-\mathbf{1 5 a}$ (3 mol\%) produced 42 different $\mathrm{N}$-alkylated aromatic amines in presence of base in moderate to high yields (Scheme 106) ${ }^{563}$ Heterocyclic products generally exhibit lower yields than carbocyclic derivatives. Tertiary amines were observed as products.

Scheme 106. $N$-alkylation of aromatic amines catalyzed by ${ }^{i \mathbf{P r}} \mathbf{M n}$ 15a, Mn-22a and Mn-1a. ${ }^{563-565}$

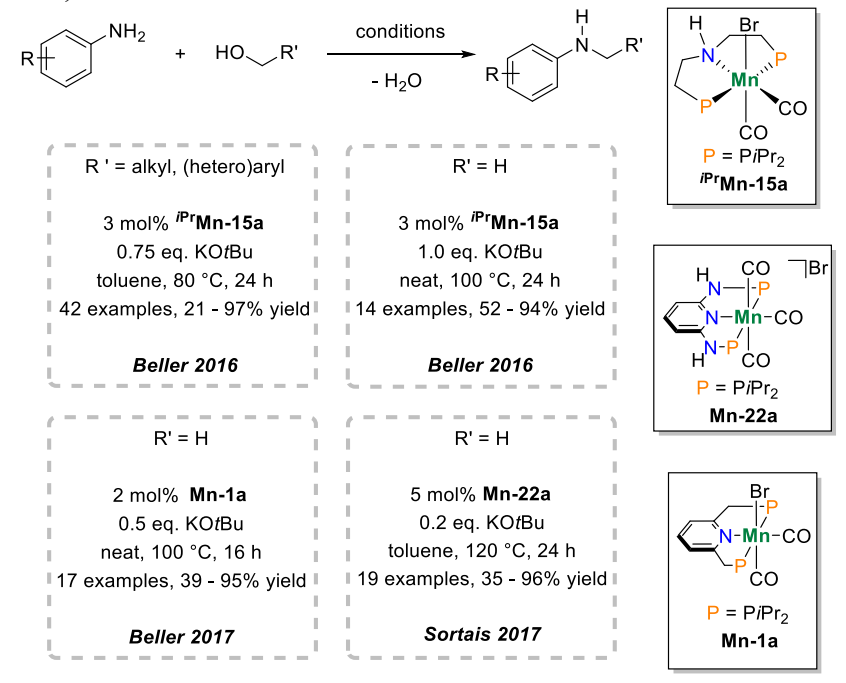

Selective primary amine $N$-methylation is particularly challenging due to more demanding dehydrogenation of methanol and a high tendency for dialkylation. The ${ }^{i \mathbf{P r}} \mathbf{M n}-15 \mathrm{a}$ catalyzed reaction allowed for $N$-methylation of 14 anilines. An improved protocol gave $N$-methylation of 17 aromatic amines with precatalyst Mn-1a at milder reaction conditions tolerating several other functional groups like ketones, C-C double bonds and amides (Scheme 106). ${ }^{564}$

A range of 19 anilines were $N$-methylated with methanol as $\mathrm{C}_{1}$ source using the manganese $\mathrm{PN}_{3} \mathrm{P}$ pincer tricarbonyl complex Mn-22a by the group of Sortais (Scheme 106). ${ }^{565}$ The reaction requires relatively low base $(0.2$ equiv. $\mathrm{KO} t \mathrm{Bu})$ but significantly higher catalyst loadings $5 \mathrm{~mol} \%$ )

Scheme 107. $\alpha$-Alkylation of ketones with primary alcohols using manganese catalyst ${ }^{i \mathbf{P r}} \mathbf{M n}-\mathbf{1 5 a} .{ }^{566}$

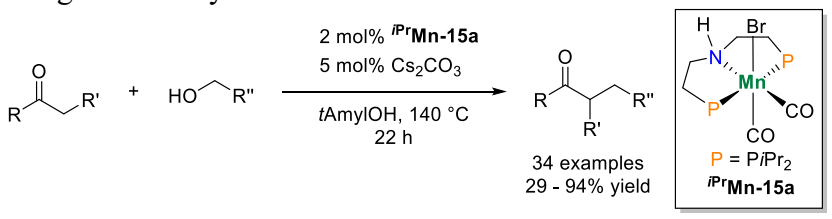

The $\alpha$-alkylation of ketones with primary alcohols was also carried out with the bifunctional manganese catalysts ${ }^{i \mathbf{P r}} \mathbf{M n}$ 15a. With $2 \mathrm{~mol} \%$ loading and activation by $\mathrm{Cs}_{2} \mathrm{CO}_{3}$ a broad range of 34 substrates could be $\alpha$-alkylated in mainly good to excellent yields (Scheme 107). ${ }^{566}$ Besides methylketones some cyclic ketones were also reported as substrates. Phenylallyl alcohol as source for the alkyl synthon gave low yields. The authors assume a typical hydrogen borrowing mechanism via $\mathrm{AAD}$, aldol condensation with the enolizable ketone and catalytic $\mathrm{C}=\mathrm{C}$ rehydrogenation. However, a rather unusual MLC path for the AAD step was proposed with $\beta$-H-transfer from an alkoxide to the pincer amide ligand of a manganese(I) ate-intermediate. This reductive elimination 
reaction leads to an unprecedented manganese(I/-I) AAD redox mechanism to rationalize the statistical deuterium distribution in the product $\alpha$ - and $\beta$-positions when starting from a $\mathrm{HOCD}_{2} \mathrm{Ph}$ isotopomer (Scheme 108). However, $\mathrm{N}-\mathrm{H} / \mathrm{Mn}-$ $\mathrm{D}$ scrambling on the manganese(I) hydride amine stage via a dihydrogen intermediate provides a more established, alternative path to rationalize the labeling experiments. A mechanistic study for this reaction is yet to be carried out.

Scheme 108. A: Proposed Mechanism for the $\alpha$-Alkylation of ketones with primary alcohols using ${ }^{i P r} \mathbf{M n - 1 5 a}$ featuring an unprecedented manganese (I/-I) AAD redox mechanism. B: Deuteration experiments using a $\mathrm{HOCD}_{2} \mathrm{Ph}$ alcohol isotopomer $\left(\mathrm{P}={\left.\mathrm{P} i \mathrm{Pr}_{2}\right) .}^{566}\right.$

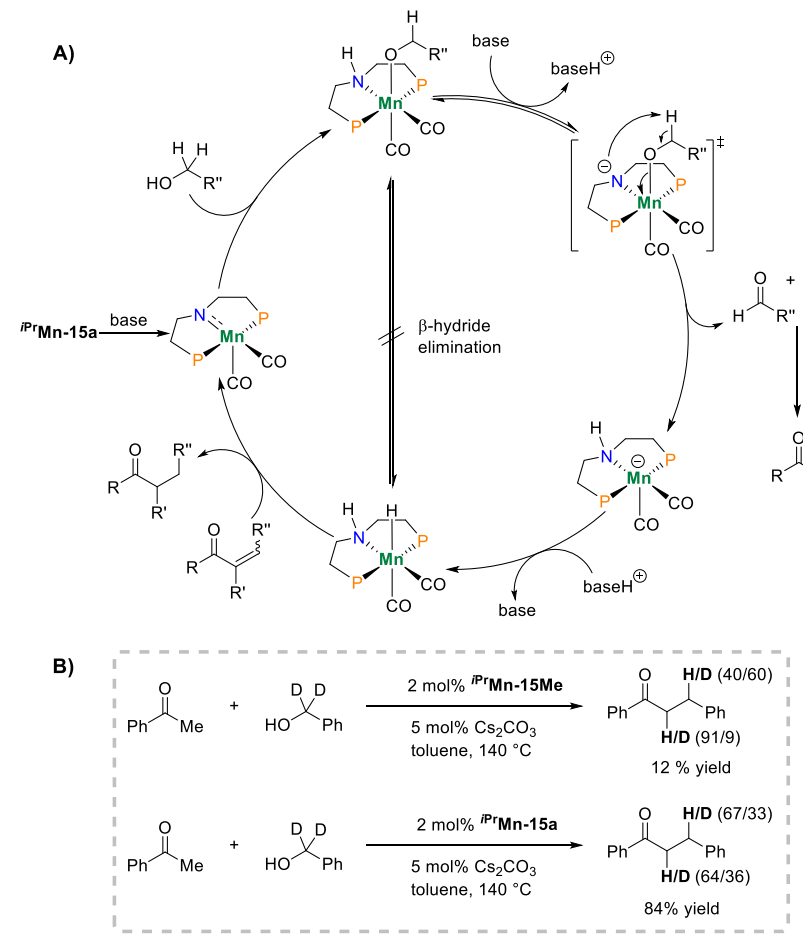

The Guerbet reaction, i.e. the $\beta$-alkylation of alcohols by homocoupling and elimination of water, represents an interesting upgrading reaction of bioavailable alcohols like ethanol to long-chain alcohols. The reaction has also been reported with bifunctional $3 \mathrm{~d}$ pincer catalysts as an extension of previously described ketone $\alpha$-alkylation that requires $\mathrm{AAD}$ of both fragments prior to aldol condensation and rehydrogenation with half of the released $\mathrm{H}_{2}$ (Scheme 109). Two groups independently utilized precatalyst ${ }^{i \operatorname{Pr}} \mathbf{M n}-15 a$ for Guerbet condensation of ethanol to butanol. ${ }^{567,568}$ The protocol established by Liu and co-workers achieved turnover numbers up to 114120 over a reaction time of $168 \mathrm{~h}$ in neat ethanol (Scheme 109) with extremely low catalyst load $(0.0001 \mathrm{~mol} \%)^{567}$ The respective backbone $N$-methylated catalyst showed significantly lower catalytic activity suggesting the relevance of MLC for the AAD cycle.
Scheme 109. Liu's protocol for Guerbet-condensation of ethanol to 1-butanol catalyzed by ${ }^{i \operatorname{Pr}} \mathbf{M n - 1 5 a}$ (A) and proposed mechanism for product formation via a hydrogen borrowing sequence $(\mathbf{B}){ }^{567}$
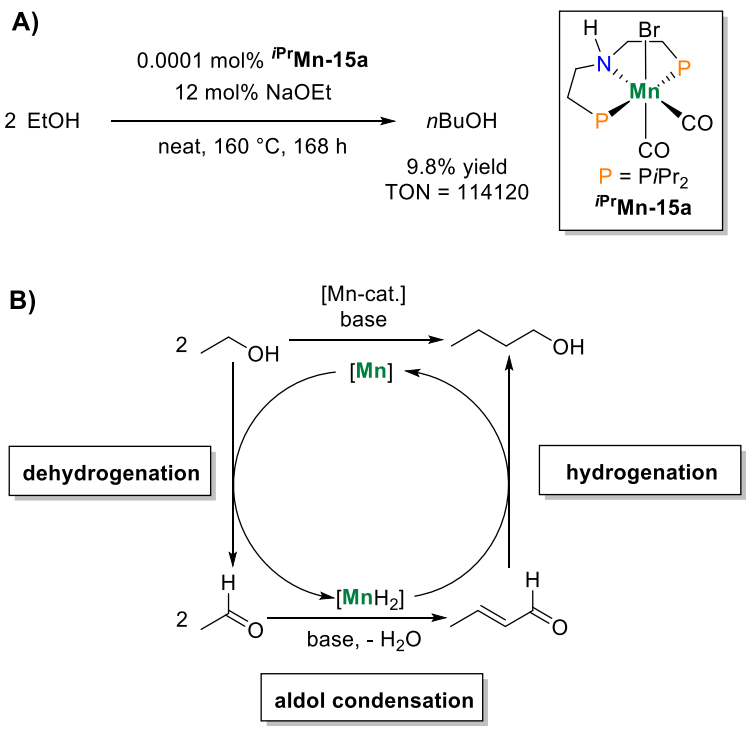

Milstein recently reported the synthesis of $N$-substituted hydrazones from alcohols and hydrazine (Scheme 110). ${ }^{569}$ $3 \mathrm{~mol} \%$ of the PNN pincer catalyst $\mathbf{M n}-\mathbf{3 3}$ were employed to obtain a range of 10 hydrazones in predominantly good yields. The dearomatized, five-coordinate $\mathrm{Mn}(\mathrm{I})$-species could be isolated and showed catalytic activity, albeit with lower yield. Reaction with benzylic alcohol generated the respective alkoxide by $\mathrm{O}-\mathrm{H}$ 1,3-addition, which formed the hydride upon heating. Based on these observations, an MLC mechanism was proposed for $\mathrm{AAD}$, which is coupled to condensation with the hydrazine and only one catalytic rehydrogenation step resulting in a remarkable selectivity for the half reduced azine product (Scheme 110).

Scheme 110. Synthesis of $N$-substituted hydrazones by manganese catalyzed coupling of alcohols with hydrazine $\left(\mathrm{P}=\mathrm{P} t \mathrm{Bu}_{2}\right) .{ }^{569}$

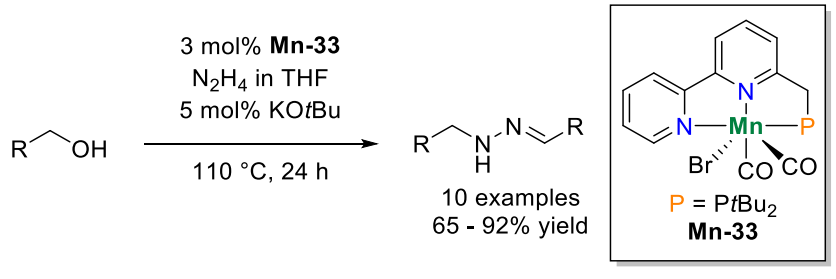

Maji and coworkers described the $\alpha$-alkylation of ketones with primary alcohols using a phosphine-free NNN pincer manganese (I) catalyst (Scheme 111). ${ }^{570}$ The acidic N-H group in the backbone can serve as proton relay by 2-hydrazinopyridine de-/aromatization, in analogy to the popular $\mathbf{P N}^{\mathbf{P y}} \mathbf{P}, \mathbf{P N}_{3} \mathbf{P}$ and $\mathbf{P N} \mathbf{N}_{5} \mathbf{P}$ ligands, enabling cooperative E-H bond heterolysis by 1,3-addition. The active catalyst was formed in situ from ligand $\mathbf{L} 1$ and $\left[\mathrm{Mn}(\mathrm{CO})_{5} \mathrm{Br}\right]$ precursors in presence of $\mathrm{KO} t \mathrm{Bu}$ and a catalytic cycle based on cooperative $\mathrm{AAD}$ was proposed. Furthermore, the dehydrogenative Friedländer synthesis of quinolines was reported (Scheme 111). 
Scheme 111. Dehydrogenative $\alpha$-alkylation of ketones with primary alcohols and Friedländer synthesis with a manganese NNN pincer catalyst and proposed mechanism. ${ }^{570}$

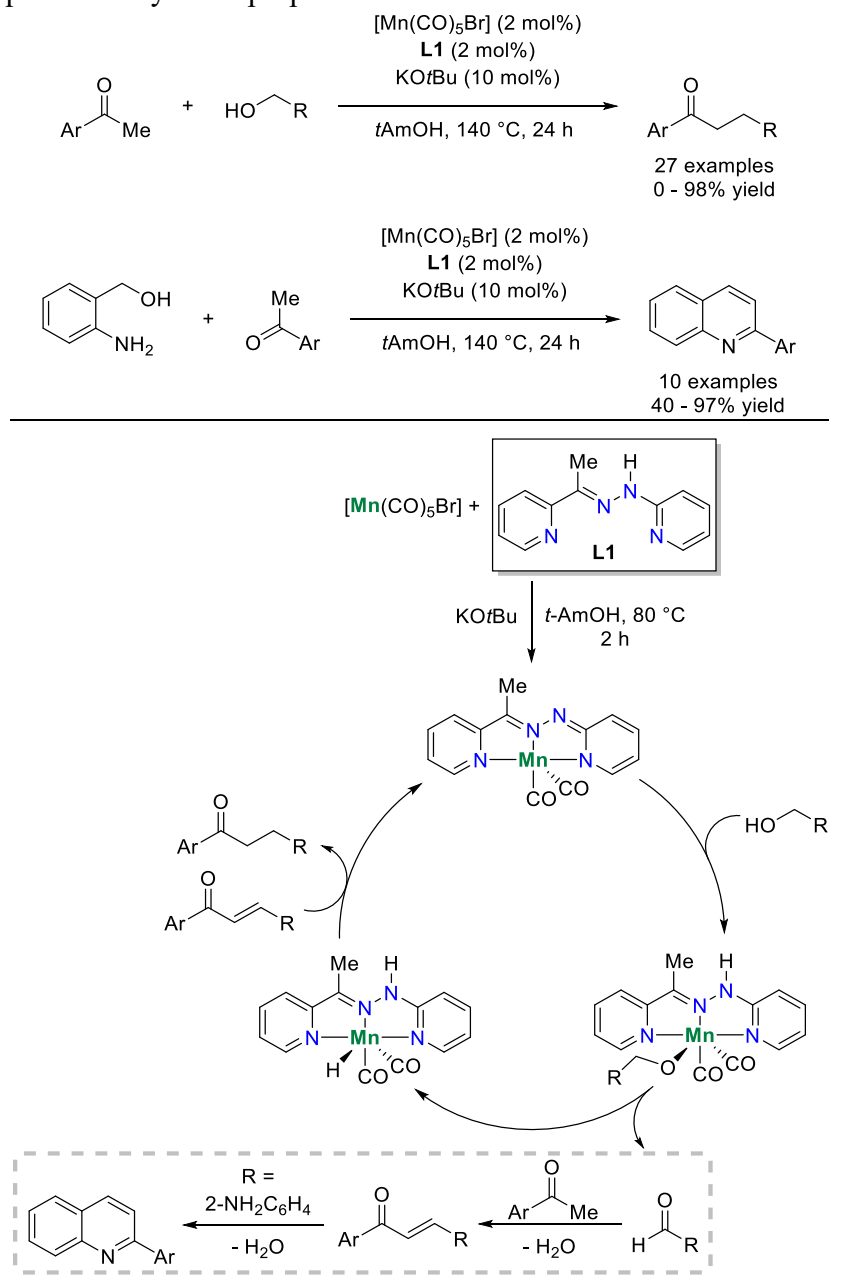

\section{OUTLOOK}

The field of $3 \mathrm{~d}$ metal pincer catalysis emerged as a competitive branch in homogeneous de-/hydrogenation catalysis over the past 10 years. The success was strongly inspired by the introduction of functional pincer ligands such as metalligand platforms that facilitate bond activation reactions by metal-ligand cooperativity. Experimental and particularly computational studies continuously refine the initial mechanistic picture. The functional pincer ligand platforms do not universally undergo reversible de-/protonation reactions in the course of catalysis. However, the conceptual impact of MLC-assisted stoichiometric bond activation continues to stimulate the design of new ligands and exploitation of base metals that were traditionally not suitable for de-/hydrogenation catalysis. These developments are to a large extend empirically driven. Future systematic studies on thermochemical and kinetic properties, such as hydride and ligand BDFE, $\mathrm{p} K_{\mathrm{a}}$ and hydricity values or hydrogen transfer rates, will be required to offer predictive tools for catalyst development and benchmarking data for computational support.

Another driving concept was the use of redox-active, weakfield pincer ligands that impose precious metal-like catalytic activity. The impact of these platforms in de-/hydrogenation is not as extensive as in other fields of catalysis such as C-C coupling. However, the emergence of systems that undergo stoichiometric and catalytic ligand-based proton coupled electron transfer complements the rapidly growing toolbox of functional pincer ligands and might lead to new applications for first-row metal catalyzed transformations.

$3 \mathrm{~d}$ metal de-/hydrogenation is dominated by iron pincer catalysts. Iron still offers the highest activities enabling in some cases catalysis with loadings in the ppm range. Suitable catalyst precursors are readily synthetically accessible and were developed as a direct extension of ruthenium and osmium catalysis. However, manganese is a 'rising star' rapidly catching up. Cobalt precatalysts with functional pincer catalysts are particularly useful for the hydrogenation of unpolar substrates, such as olefins. Their open-shell electronic states hampers the development of a comprehensive mechanistic picture to the same extend as for iron and manganese leaving ample room for future work. The expansion of the isoelectronic iron(II) and manganese(I) series to cobalt(III) or chromium(0) will be challenging goals for catalyst design.

The current catalytic scope focuses on the transformation of polar substrates that can be activated by metal-ligand cooperative pincer platforms. In that context, several frontiers still remain, such as the hydrogenation of $\mathrm{CO}_{2}$ beyond formate to methanol or even methane. The direct hydrogenation or carbonic acids provides another challenging task. Catalytic alkane dehydrogenation, which strongly benefitted form the application of pincer ligands, is still confined to precious metal catalysis. And certainly the catalytic reduction of dinitrogen, which is carried out industrially in massive scale with a heterogeneous iron catalyst, is waiting for an efficient catalytic solution at ambient conditions. The introduction of functional pincer ligands that facilitate proton coupled electron transfer to such challenging substrates offers strategies for the development of thermal, electrochemical and photochemical catalysts.

\section{AUTHOR INFORMATION}

\section{Corresponding Author}

*E-mail: sven.schneider@chemie.uni-goettingen.de

\section{Author Contributions}

* L.A. and M.F. contributed equally to this work.

\section{Biographies}

Lukas Alig (born in 1992) obtained his undergraduate education at the Philipps-Universität Marburg, Germany, and graduated with a master thesis in the group of Dr. R. Langer. He subsequently joined the group of Prof. Sven Schneider at the Georg-August-Universität Göttingen working as a Ph.D. student on small molecule activation with group 7 pincer complexes.

Maximilian Fritz (born in 1989) is a Ph.D. student in the group of Prof. Sven Schneider at Georg-August Universität, Göttingen. He previously studied at Philipps-Universität Marburg and obtained a master degree in the group of Dr. R. Langer. His research interests include the activation of small molecules with chromium pincer complexes. 
Sven Schneider (born in 1972) is the managing director of the Institute for Inorganic Chemistry at the Georg-August Universität in Göttingen and holds a chair for Functional Supramolecular Chemistry. He studied chemistry at the Technische Universität Darmstadt and obtained a diploma in 1999 under the supervision of Prof. H.-J. Klein. He received a Ph.D. from the Humboldt Unversität zu Berlin under the supervision of Prof. A.C. Filippou in 2003. After Postdoctoral research in the group of Prof. T.J. Marks at the Northwestern University he started his independent career as an Emmy-Noether group leader at Technische Universität München. In 2010 he moved to Friedrich-Alexander-Universität Erlangen-Nürnberg as Associate Professor for Inorganic Chemistry and assumed his current position in Göttingen in 2012. His research interests include organometallic chemistry, small molecule activation, homogeneous catalysis and materials synthesis by gas-phase deposition techniques.

\section{ACKNOWLEDGMENT}

This paper is dedicated to Prof. Dr. Dietmar Stalke on the occasion of his $60^{\text {th }}$ birthday. S.Sch. acknowledges to the European Research Council (ERC Grant Agreement 646747) for funding.

\section{REFERENCES}

(1) Tzouras, N. V.; Stamatopoulos, I. K.; Papastavrou, A. T.; Liori, A. A.; Vougioukalakis, G. C. Sustainable Metal Catalysis in C-H Activation. Coord. Chem. Rev. 2017, 343, 25138.

(2) van der Vlugt, J. I.; Reek, J. N. H. Neutral Tridentate PNP Ligands and Their Hybrid Analogues: Versatile NonInnocent Scaffolds for Homogeneous Catalysis. Angew. Chem. Int. Ed. 2009, 48, 8832-8846.

(3) Singh, A.; Spiccia, L. Water Oxidation Catalysts Based on Abundant 1st Row Transition Metals. Coord. Chem. Rev. 2013, 257, 2419.

(4) Bauer, I.; Knölker, H.-J. Iron Catalysis in Organic Synthesis. Chem. Rev. 2015, 115, 3170-3387.

(5) Pellissier, H.; Clavier, H. Enantioselective Cobalt-Catalyzed Transformations. Chem. Rev. 2014, 114, 2775-2823.

(6) Gupta, K. C.; Sutar, A. K. Catalytic Activities of Schiff Base Transition Metal Complexes. Coord. Chem. Rev. 2008, 252, 1420-1450.

(7) McGarrigle, E. M.; Gilheany, D. G. Chromium- and Manganese-salen Promoted Epoxidation of Alkenes. Chem. Rev. 2005, 105, 1563-1602.

(8) Asay, M.; Morales-Morales, D. Non-Symmetric Pincer Ligands: Complexes and Applications in Catalysis. Dalton Trans. 2015, 44, 17432-17447.

(9) Crossley, S. W. M.; Obradors, C.; Martinez, R. M.; Shenvi, R. A. Mn-, Fe-, and Co-Catalyzed Radical Hydrofunctionalizations of Olefins. Chem. Rev. 2016, 116, 8912-9000.

(10) Dai, H.; Guan, H. Iron Dihydride Complexes: Synthesis, Reactivity, and Catalytic Applications. Isr. J. Chem. 2017, 57, 1170-1203.

(11) Deng, Q. H.; Melen, R. L.; Gade, L. H. Anionic Chiral Tridentate N-Donor Pincer Ligands in Asymmetric Catalysis. Acc. Chem. Res. 2014, 47, 3162-3173.

(12) Valyaev, D. A.; Lavigne, G.; Lugan, N. Manganese Organometallic Compounds in Homogeneous Catalysis: Past, Present, and Prospects. Coord. Chem. Rev. 2016, 308, $191-235$.

(13) Riener, K.; Haslinger, S.; Raba, A.; Högerl, M. P.; Cokoja, M.; Herrmann, W. A.; Kühn, F. E. Chemistry of Iron N Heterocyclic Carbene Complexes: Syntheses, Structures, Reactivities, and Catalytic Applications. Chem. Rev. 2014, 114,
5215-5272.

(14) Mérel, D. S.; Do, M. L. T.; Gaillard, S.; Dupau, P.; Renaud, J. L. Iron-Catalyzed Reduction of Carboxylic and Carbonic Acid Derivatives. Coord. Chem. Rev. 2015, 288, 50-68.

(15) Fürstner, A. Iron Catalysis in Organic Synthesis: A Critical Assessment of What It Takes to Make This Base Metal a Multitasking Champion. ACS Cent. Sci. 2o16, 2, 778-789.

(16) Zweig, J. E.; Kim, D. E.; Newhouse, T. R. Methods Utilizing First-Row Transition Metals in Natural Product Total Synthesis. Chem. Rev. 2017, 117, 11680-11752.

(17) Li, Y.-Y.; Yu, S.-L.; Shen, W.-Y.; Gao, J.-X. Iron-, Cobalt-, and Nickel-Catalyzed Asymmetric Transfer Hydrogenation and Asymmetric Hydrogenation of Ketones. Acc. Chem. Res. 2015, 48, 2587-2598.

(18) Chakraborty, S.; Bhattacharya, P.; Dai, H.; Guan, H. Nickel and Iron Pincer Complexes as Catalysts for the Reduction of Carbonyl Compounds. Acc. Chem. Res. 2015, 48, 1995-2003.

(19) Su, B.; Cao, Z.-C.; Shi, Z.-J. Exploration of Earth-Abundant Transition Metals ( $\mathrm{Fe}, \mathrm{Co}$, and $\mathrm{Ni}$ ) as Catalysts in Unreactive Chemical Bond Activations. Acc. Chem. Res. 2015, 48, 886896.

(20) Kallmeier, F.; Kempe, R. Manganese Complexes for (De)Hydrogenation Catalysis: A Comparison to Cobalt and Iron Catalysts. Angew. Chem. Int. Ed. 2018, 57, 46-6o.

(21) Garbe, M.; Junge, K.; Beller, M. Homogeneous Catalysis by Manganese-Based Pincer Complexes. Eur. J. Org. Chem. 2017, 2017, 4344-4362.

(22) Belkova, N. V.; Epstein, L. M.; Filippov, O. A.; Shubina, E. S. Hydrogen and Dihydrogen Bonds in the Reactions of Metal Hydrides. Chem. Rev. 2016, 116, 8545-8587.

(23) Rossin, A.; Peruzzini, M. Ammonia-Borane and AmineBorane Dehydrogenation Mediated by Complex Metal Hydrides. Chem. Rev. 2016, 116, 8848-8872.

(24) Eberhardt, N. A.; Guan, H. Nickel Hydride Complexes. Chem. Rev. 2016, 116, 8373-8426.

(25) Bauer, G.; Hu, X. Recent Developments of Iron Pincer Complexes for Catalytic Applications. Inorg. Chem. Front. 2016, 3, 741-765.

(26) Maji, B.; Barman, M. Recent Developments of Manganese Complexes for Catalytic Hydrogenation and Dehydrogenation Reactions. Synthesis 2017, 49, 3377-3393.

(27) Filonenko, G. A.; van Putten, R.; Hensen, E. J. M.; Pidko, E. A. Catalytic (De)hydrogenation Promoted by Non-Precious Metals - Co, Fe and Mn: Recent Advances in an Emerging Field. Chem. Soc. Rev. 2018, 47, 1459-1483.

(28) Chen, X.; Yang, X. Mechanistic Insights and Computational Design of Transition-Metal Catalysts for Hydrogenation and Dehydrogenation Reactions. Chem. Rec. 2016, 16, 2364-2378.

(29) Bullock, R. M.; Helm, M. L. Molecular Electrocatalysts for Oxidation of Hydrogen Using Earth-Abundant Metals: Shoving Protons Around with Proton Relays. Acc. Chem. Res. 2015, 48, 2017-2026.

(30) Werkmeister, S.; Neumann, J.; Junge, K.; Beller, M. PincerType Complexes for Catalytic (De)Hydrogenation and Transfer (De)Hydrogenation Reactions: Recent Progress. Chem. Eur. J. 2015, 21, 12226-12250.

(31) Wang, D.; Astruc, D. The Golden Age of Transfer Hydrogenation. Chem. Rev. 2015, 115, 6621-6686.

(32) Crabtree, R. H. Homogeneous Transition Metal Catalysis of Acceptorless Dehydrogenative Alcohol Oxidation: Applications in Hydrogen Storage and to Heterocycle Synthesis. Chem. Rev. 2017, 117, 9228-9246.

(33) Bernskoetter, W. H.; Hazari, N. Reversible Hydrogenation of Carbon Dioxide to Formic Acid and Methanol: Lewis Acid Enhancement of Base Metal Catalysts. Acc. Chem. Res. 2o17, 50, 1049-1058.

(34) Trovitch, R. J. The Emergence of Manganese-Based Carbonyl Hydrosilylation Catalysts. Acc. Chem. Res. 2017, 50, 28422852.

(35) Du, X.; Huang, Z. Advances in Base-Metal-Catalyzed Alkene Hydrosilylation. ACS Catal. 2017, 7, 1227-1243. 
Obligacion, J. V.; Chirik, P. J. Earth-Abundant Transition Metal Catalysts for Alkene Hydrosilylation and Hydroboration. Nat. Rev. Chem. 2018, 2, 15-34.

(37) Greenhalgh, M. D.; Jones, A. S.; Thomas, S. P. Iron-Catalysed Hydrofunctionalisation of Alkenes and Alkynes; 2015; Vol. 7.

(38) Yoshida, H. Borylation of Alkynes under Base/Coinage Metal Catalysis: Some Recent Developments. ACS Catal. 2016, 6, 1799-1811.

(39) Sordakis, K.; Tang, C.; Vogt, L. K.; Junge, H.; Dyson, P. J.; Beller, M.; Laurenczy, G. Homogeneous Catalysis for Sustainable Hydrogen Storage in Formic Acid and Alcohols. Chem. Rev. 2018, 118, 372-433.

(40) Mellmann, D.; Sponholz, P.; Junge, H.; Beller, M. Formic Acid as a Hydrogen Storage Material - Development of Homogeneous Catalysts for Selective Hydrogen Release. Chem. Soc. Rev. 2016, 45, 3954-3988.

(41) Thoi, V. S.; Sun, Y.; Long, J. R.; Chang, C. J. Complexes of Earth-Abundant Metals for Catalytic Electrochemical Hydrogen Generation under Aqueous Conditions. Chem. Soc. Rev. 2013, 42, 2388-2400.

(42) Rakowski DuBois, M.; DuBois, D. L. The Roles of the First and Second Coordination Spheres in the Design of Molecular Catalysts for $\mathrm{H}_{2}$ Production and Oxidation. Chem. Soc. Rev. 2009, 38, 62-72.

(43) Fukuzumi, S.; Lee, Y. M.; Nam, W. Thermal and Photocatalytic Production of Hydrogen with EarthAbundant Metal Complexes. Coord. Chem. Rev. 2018, 355, 54-73.

(44) Müller, K.; Brooks, K.; Autrey, T. Hydrogen Storage in Formic Acid: A Comparison of Process Options. Energy $\mathcal{E}$ Fuels 2017, 31, 12603-12611.

(45) Preuster, P.; Papp, C.; Wasserscheid, P. Liquid Organic Hydrogen Carriers (LOHCs): Toward a Hydrogen-Free Hydrogen Economy. Acc. Chem. Res. 2017, 50, 74-85.

(46) Zell, T.; Langer, R. Iron-Catalyzed Hydrogenation and Dehydrogenation Reactions with Relevance to Reversible Hydrogen Storage Applications. Recycl. Catal. 2016, 2, 87109.

(47) Egorova, K. S.; Ananikov, V. P. Which Metals Are Green for Catalysis? Comparison of the Toxicities of $\mathrm{Ni}, \mathrm{Cu}, \mathrm{Fe}, \mathrm{Pd}, \mathrm{Pt}$, Rh, and Au Salts. Angew. Chem. Int. Ed. 2016, 55, 12150-12162.

(48) Wedepohl, K. H. The Composition of the Continental Crust. Geochim. Cosmochim. Acta 1995, 59, 1217-1232.

(49) Moulton, C. J.; Shaw, B. L. Transition Metal-carbon Bonds. Part XLII. Complexes of Nickel, Palladium, Platinum, Rhodium and Iridium with the Tridentate Ligand 2,6Bis[(di-T-Butylphosphino)methyl $]$ phenyl. J. Chem. Soc., Dalton Trans. 1976, No. 11, 1020-1024.

(50) Albrecht, M.; van Koten, G. Platinum Group Organometallics Based on "Pincer" Complexes: Sensors, Switches, and Catalysts. Angew. Chem. Int. Ed. 2001, 40, 3750-3781.

(51) Gunanathan, C.; Milstein, D. Bond Activation and Catalysis by Ruthenium Pincer Complexes. Chem. Rev. 2014, 114, 12024-12087.

(52) Schneider, S.; Meiners, J.; Askevold, B. Cooperative Aliphatic PNP Amido Pincer Ligands - Versatile Building Blocks for Coordination Chemistry and Catalysis. Eur. J. Inorg. Chem. 2012, 2012, 412-429.

(53) Choi, J.; MacArthur, A. H. R.; Brookhart, M.; Goldman, A. S. Dehydrogenation and Related Reactions Catalyzed by Iridium Pincer Complexes. Chem. Rev. 2o11, 111, 1761-1779.

(54) Peris, E.; Crabtree, R. H. Key Factors in Pincer Ligand Design. Chem. Soc. Rev. 2018, 47, 1959-1968.

(55) Luca, O. R.; Crabtree, R. H. Redox-Active Ligands in Catalysis. Chem. Soc. Rev. 2013, 42, 1440-1459.

(56) van Koten, G. Tuning the Reactivity of Metals Held in a Rigid Ligand Environment. Pure Appl. Chem. 1989, 61, 1681-1694.

(57) Valdés, H.; García-Eleno, M. A.; Canseco-Gonzalez, D.; Morales-Morales, D. Recent Advances in Catalysis with Transition-Metal Pincer Compounds. ChemCatChem 2o18,
10, 3136-3172.

(58) van Koten, G.; Milstein, D. Organometallic Pincer Chemistry; Springer, 2013.

(59) van Koten, G.; Gossage, R. A. The Privileged Pincer-Metal Platform: Coordination Chemistry Eamp; Applications; Springer, 2016.

(6o) Morales-Morales, D. Pincer Compounds; Elsevier, 2018.

(61) O'Reilly, M. E.; Veige, A. S. Trianionic Pincer and PincerType Metal Complexes and Catalysts. Chem. Soc. Rev. 2014, 43, 6325-6369.

(62) Murugesan, S.; Kirchner, K. Non-Precious Metal Complexes with an Anionic PCP Pincer Architecture. Dalton Trans. 2016, 45, 416-439.

(63) Benito-Garagorri, D.; Kirchner, K. Modularly Designed Transition Metal PNP and PCP Pincer Complexes Based on Aminophosphines: Synthesis and Catalytic Applications. Acc. Chem. Res. 2008, 41, 201-213.

(64) Pugh, D.; Danopoulos, A. A. Metal Complexes with "pincer"Type Ligands Incorporating N-Heterocyclic Carbene Functionalities. Coord. Chem. Rev. 2007, 251, 610-641.

(65) Zell, T.; Langer, R. From Ruthenium to Iron and ManganeseA Mechanistic View on Challenges and Design Principles of Base-Metal Hydrogenation Catalysts. ChemCatChem 2018, 10, 1930-1940.

(66) Warren, J. J.; Tronic, T. A.; Mayer, J. M. Thermochemistry of Proton-Coupled Electron Transfer Reagents and Its Implications. Chem. Rev. 2010, 110, 6961-7001.

(67) Morris, R. H. Brønsted-Lowry Acid Strength of Metal Hydride and Dihydrogen Complexes. Chem. Rev. 2016, 116, 8588-8654.

(68) Wiedner, E. S.; Chambers, M. B.; Pitman, C. L.; Bullock, R. M.; Miller, A. J. M.; Appel, A. M. Thermodynamic Hydricity of Transition Metal Hydrides. Chem. Rev. 2o16, 116, 86558692.

(69) Suarez, A. I. O.; Lyaskovskyy, V.; Reek, J. N. H.; van der Vlugt, J. I.; de Bruin, B. Complexes with Nitrogen-Centered Radical Ligands: Classification, Spectroscopic Features, Reactivity, and Catalytic Applications. Angew. Chem. Int. Ed. 2013, 52, 12510-12529.

(70) Praneeth, V. K. K.; Ringenberg, M. R.; Ward, T. R. RedoxActive Ligands in Catalysis. Angew. Chem. Int. Ed. 2012, 51, 10228-10234.

(71) Blanchard, S.; Derat, E.; Desage-El Murr, M.; Fensterbank, L.; Malacria, M.; Mouriès-Mansuy, V. Non-Innocent Ligands: New Opportunities in Iron Catalysis. Eur. J. Inorg. Chem. 2012, 2012, 376-389.

(72) de Bruin, B.; Gualco, P.; Paul, N. D. Redox Non-Innocent Ligands. In Ligand Design in Metal Chemistry; John Wiley \& Sons, Ltd: Chichester, UK, 2016; pp 176-204.

(73) Winter, A.; Newkome, G. R.; Schubert, U. S. Catalytic Applications of Terpyridines and Their Transition Metal Complexes. ChemCatChem 2o11, 3, 1384-1406.

(74) Elgrishi, N.; Chambers, M. B.; Wang, X.; Fontecave, M. Molecular Polypyridine-Based Metal Complexes as Catalysts for the Reduction of $\mathrm{CO}_{2}$. Chem. Soc. Rev. 2017, 46, 761-796.

(75) Karunananda, M. K.; Mankad, N. P. Cooperative Strategies for Catalytic Hydrogenation of Unsaturated Hydrocarbons. ACS Catal. 2017, 7, 6110-6119.

(76) Li, H.; Zheng, B.; Huang, K.-W. A New Class of PN3-Pincer Ligands for Metal-ligand Cooperative Catalysis. Coord. Chem. Rev. 2015, 293-294, 116-138.

(77) Morris, R. H. Exploiting Metal-Ligand Bifunctional Reactions in the Design of Iron Asymmetric Hydrogenation Catalysts. Acc. Chem. Res. 2015, 48, 1494-1502.

(78) Moore, C. M.; Szymczak, N. K. Appended Functionality in Pincer Ligands. In Pincer and Pincer-Type Complexes: Applications in Organic Synthesis and Catalysis; 2014; Vol. 9783527334, pp 71-94.

(79) Tilset, M.; Parker, V. D. Solution Homolytic Bond Dissociation Energies of Organotransition-Metal Hydrides. J. Am. Chem. Soc. 1989, 111, 6711-6717. 

Transition Metal Hydrides: Hydrogen Atom, Hydride, and Proton Transfer Reactions. Comments Inorg. Chem. 1991, 12, $1-33$.

(81) Bullock, R. M. Catalytic Ionic Hydrogenations. Chem. Eur. J. 2004, 10, 2366-2374.

(82) Morris, R. H. Estimating the Acidity of Transition Metal Hydride and Dihydrogen Complexes by Adding Ligand Acidity Constants. J. Am. Chem. Soc. 2014, 136, 1948-1959.

(83) Curtis, C. J.; Miedaner, A.; Raebiger, J. W.; DuBois, D. L. Periodic Trends in Metal Hydride Donor Thermodynamics: Measurement and Comparison of the Hydride Donor Abilities of the Series HM(PNP) $2+(\mathrm{M}=\mathrm{Ni}, \mathrm{Pd}, \mathrm{Pt}$; PNP $=$ Et 2 PCH $2 \mathrm{~N}(\mathrm{Me}) \mathrm{CH} 2 \mathrm{PEt} 2$ ). Organometallics 2004, 23, 511-516.

(84) Ciancanelli, R.; Noll, B. C.; DuBois, D. L.; DuBois, M. R. Comprehensive Thermodynamic Characterization of the Metal-Hydrogen Bond in a Series of Cobalt-Hydride Complexes. J. Am. Chem. Soc. 2002, 124, 2984-2992.

(85) Wilson, A. D.; Miller, A. J. M.; DuBois, D. L.; Labinger, J. A.; Bercaw, J. E. Thermodynamic Studies of $\left[\begin{array}{ll}\mathrm{H} & 2\end{array}\right.$ $\mathrm{Rh}$ (diphosphine) 2 ] + and [HRh(diphosphine) 2 ( $\left.\left.\mathrm{CH}_{3} \mathrm{CN}\right)\right]$ 2+ Complexes in Acetonitrile. Inorg. Chem. 2010, 49, 39183926.

(86) Price, A. J.; Ciancanelli, R.; Noll, B. C.; Curtis, C. J.; DuBois, D. L.; DuBois, M. R. HRh(dppb) 2 , a Powerful Hydride Donor. Organometallics 2002, 21, 4833-4839.

(87) Mock, M. T.; Potter, R. G.; O’Hagan, M. J.; Camaioni, D. M.; Dougherty, W. G.; Kassel, W. S.; DuBois, D. L. Synthesis and Hydride Transfer Reactions of Cobalt and Nickel Hydride Complexes to BX 3 Compounds. Inorg. Chem. 2011, 50, 1191411928.

(88) Sandhya, K. S.; Suresh, C. H. Quantification of Thermodynamic Hydridicity of Hydride Complexes of Mn, Re, Mo, and W Using the Molecular Electrostatic Potential. J. Phys. Chem. A 2017, 121, 2814-2819.

(89) Waldie, K. M.; Ostericher, A. L.; Reineke, M. H.; Sasayama, A. F.; Kubiak, C. P. Hydricity of Transition-Metal Hydrides: Thermodynamic Considerations for $\mathrm{CO}_{2}$ Reduction. ACS Catal. 2018, 8, 1313-1324.

(9o) Abbel, R.; Abdur-Rashid, K.; Faatz, M.; Hadzovic, A.; Lough, A. J.; Morris, R. H. A Succession of Isomers of Ruthenium Dihydride Complexes. Which One Is the Ketone Hydrogenation Catalyst? J. Am. Chem. Soc. 2005, 127, 18701882.

(91) Chen, Z.; Chen, Y.; Tang, Y.; Lei, M. A Theoretical Study of X Ligand Effect on Catalytic Activity of Complexes RuHX(diamine)(PPh3)2 (X = NCMe, CO, Cl, OMe, OPh, CCMe and $\mathrm{H}$ ) in $\mathrm{H}_{2}$-Hydrogenation of Ketones. Dalton Trans. 2010, 39, 2036.

(92) Conley, B. L.; Pennington-Boggio, M. K.; Boz, E.; Williams, T. J. Discovery, Applications, and Catalytic Mechanisms of Shvo's Catalyst. Chem. Rev. 2010, 110, 2294-2312.

(93) Noyori, R.; Ohkuma, T. Asymmetric Catalysis by Architectural and Functional Molecular Engineering: Practical Chemo- and Stereoselective Hydrogenation of Ketones. Angew. Chem. Int. Ed. 2001, 40, 40-73.

(94) Hems, W. P.; Groarke, M.; Zanotti-Gerosa, A.; Grasa, G. A. [(Bisphosphine) $\mathrm{Ru}(\mathrm{II})$ Diamine] Complexes in Asymmetric Hydrogenation: Expanding the Scope of the Diamine Ligand. Acc. Chem. Res. 2007, 40, 1340-1347.

(95) Sandoval, C. A.; Ohkuma, T.; Muñiz, K.; Noyori, R. Mechanism of Asymmetric Hydrogenation of Ketones Catalyzed by BINAP/1,2-Diamine-Ruthenium(II) Complexes. J. Am. Chem. Soc. 2003, 125, 13490-13503.

(96) Abdur-Rashid, K.; Clapham, S. E.; Hadzovic, A.; Harvey, J. N.; Lough, A. J.; Morris, R. H. Mechanism of the Hydrogenation of Ketones Catalyzed by TransDihydrido(diamine)ruthenium(II) Complexes †. J. Am. Chem. Soc. 2002, 124, 15104-15118.

(97) Clapham, S. E.; Hadzovic, A.; Morris, R. H. Mechanisms of the H2-Hydrogenation and Transfer Hydrogenation of Polar Bonds Catalyzed by Ruthenium Hydride Complexes. Coord. Chem. Rev. 2004, 248, 2201-2237.

(98) Muñiz, K. Bifunctional Metal-Ligand Catalysis: Hydrogenations and New Reactions within the Metal(Di)amine Scaffold. Angew. Chem. Int. Ed. 2005, 44, 66226627.

(99) Samec, J. S. M.; Bäckvall, J.-E.; Andersson, P. G.; Brandt, P. Mechanistic Aspects of Transition Metal-Catalyzed Hydrogen Transfer Reactions. Chem. Soc. Rev. 2006, 35, 237.

(10o) Ikariya, T.; Blacker, A. J. Asymmetric Transfer Hydrogenation of Ketones with Bifunctional Transition Metal-Based Molecular Catalysts †. Acc. Chem. Res. 2007, 40, 1300-1308.

(101) Ito, M.; Ikariya, T. Catalytic Hydrogenation of Polar Organic Functionalities Based on Ru-Mediated Heterolytic Dihydrogen Cleavage. Chem. Commun. 2007, o, 5134.

(102) Dub, P. A.; Henson, N. J.; Martin, R. L.; Gordon, J. C. Unravelling the Mechanism of the Asymmetric Hydrogenation of Acetophenone by $\left[\begin{array}{ll}\mathrm{RuX} & 2\end{array}\right.$ (diphosphine)(1,2-Diamine)] Catalysts. J. Am. Chem. Soc. 2014, 136, 3505-3521.

(103) Dub, P. A.; Gordon, J. C. The Mechanism of Enantioselective Ketone Reduction with Noyori and Noyori-Ikariya Bifunctional Catalysts. Dalton Trans. 2016, 45, 6756-6781.

(104) Trincado, M.; Grützmacher, H. Cooperating Ligands in Catalysis. In Cooperative Catalysis; Wiley-VCH Verlag GmbH \& Co. KGaA: Weinheim, Germany, 2015; Vol. 47, pp 67-110.

(105) Grützmacher, H. Cooperating Ligands in Catalysis. Angew. Chem. Int. Ed. 2008, 47, 1814-1818.

(106) Khusnutdinova, J. R.; Milstein, D. Metal-Ligand Cooperation. Angew. Chem. Int. Ed. 2015, 54, 12236-12273.

(107) Langer, R.; Leitus, G.; Ben-David, Y.; Milstein, D. Efficient Hydrogenation of Ketones Catalyzed by an Iron Pincer Complex. Angew. Chem. Int. Ed. 2011, 50, 2120-2124.

(108) Gunanathan, C.; Milstein, D. Metal-Ligand Cooperation by Aromatization-Dearomatization: A New Paradigm in Bond Activation and "Green" Catalysis. Acc. Chem. Res. 2011, 44, 588-602.

(109) Milstein, D. Metal-Ligand Cooperation by AromatizationDearomatization as a Tool in Single Bond Activation. Philos. Trans. R. Soc. A Math. Phys. Eng. Sci. 2015, 373, 2014018920140189.

(110) Schirmer, W.; Flörke, U.; Haupt, H.-J. Zur Charakterisierung von Eigenschaften Der Versteiften Dreizähnigen Aminophosphanliganden N,N'-Bis(diphenylphosphino)-2,6Diaminopyridin Und N,N-Bis(diphenylphosphino)-2Aminopyridin Mit Metallen Der Chromgruppe. Z. Anorg. Allg. Chem. 1989, 574, 239-255.

(111) Benito-Garagorri, D.; Becker, E.; Wiedermann, J.; Lackner, W.; Pollak, M.; Mereiter, K.; Kisala, J.; Kirchner, K. Achiral and Chiral Transition Metal Complexes with Modularly Designed Tridentate PNP Pincer-Type Ligands Based on NHeterocyclic Diamines. Organometallics 20o6, 25, 1900-1913.

(112) Dauth, A.; Gellrich, U.; Diskin-Posner, Y.; Ben-David, Y.; Milstein, D. The Ferraquinone-Ferrahydroquinone Couple: Combining Quinonic and Metal-Based Reactivity. J. Am. Chem. Soc. 2017, 139, 2799-2807.

(113) Gorgas, N.; Kirchner, K. Isoelectronic Manganese and Iron Hydrogenation/Dehydrogenation Catalysts: Similarities and Divergences. Acc. Chem. Res. 2018, 51, 1558-1569.

(114) Liu, W.; Sahoo, B.; Junge, K.; Beller, M. Cobalt Complexes as an Emerging Class of Catalysts for Homogeneous Hydrogenations. Acc. Chem. Res. 2018, acs.accounts.8boo262.

(115) Poitras, A. M.; Knight, S. E.; Bezpalko, M. W.; Foxman, B. M.; Thomas, C. M. Addition of $\mathrm{H} 2$ Across a Cobalt-Phosphorus Bond. Angew. Chem. Int. Ed. 2018, 57, 1497-1500.

(116) Gutsulyak, D. V.; Piers, W. E.; Borau-Garcia, J.; Parvez, M. Activation of Water, Ammonia, and Other Small Molecules 
by PC Carbene P Nickel Pincer Complexes. J. Am. Chem. Soc. 2o13, 135, 11776-11779.

(117) Liu, C.-C.; Liu, Q.-L.; Wu, Z.-Y.; Chen, Y.-C.; Xie, H.-J.; Lei, Q.-F.; Fang, W.-J. Mechanistic Insights into Small Molecule Activation Induced by Ligand Cooperativity in PCcarbeneP Nickel Pincer Complexes: A Quantum Chemistry Study. J. Mol. Model. 2015, 21, 242.

(118) Borau-Garcia, J.; Gutsulyak, D. V; Burford, R. J.; Piers, W. E. Selective Hydration of Nitriles to Amides Catalysed by PCP Pincer Supported nickel(II) Complexes. Dalton Trans. 2015, 44, 12082-12085.

(119) LaPierre, E. A.; Piers, W. E.; Spasyuk, D. M.; Bi, D. W Activation of $\mathrm{Si}-\mathrm{H}$ Bonds across the Nickel Carbene Bond in Electron Rich Nickel PC Carbene P Pincer Complexes. Chem. Commun. 2016, 52, 1361-1364.

(120) He, T.; Tsvetkov, N. P.; Andino, J. G.; Gao, X.; Fullmer, B. C.; Caulton, K. G. Mechanism of Heterolysis of $\mathrm{H} 2$ by an Unsaturated D 8 Nickel Center: Via Tetravalent Nickel? J. Am. Chem. Soc. 2010, 132, 910-911.

(121) Lin, T.-P.; Peters, J. C. Boryl-Metal Bonds Facilitate Cobalt/Nickel-Catalyzed Olefin Hydrogenation. J. Am. Chem. Soc. 2014, 136, 13672-13683.

(122) Dzik, W. I.; Van Der Vlugt, J. I.; Reek, J. N. H.; De Bruin, B. Ligands That Store and Release Electrons during Catalysis. Angew. Chem. Int. Ed. 2011, 50, 3356-3358.

(123) Halpern, J. Oxidative-Addition Reactions of Transition Metal Complexes. Acc. Chem. Res. 1970, 3, 386-392.

(124) Ryabov, A. D. Mechanisms of Intramolecular Activation of C-H Bonds in Transition-Metal Complexes. Chem. Rev. 1990, 90, 403-424.

(125) Chirik, P. J. Carbon-Carbon Bond Formation in a Weak Ligand Field: Leveraging Open-Shell First-Row TransitionMetal Catalysts. Angew. Chem. Int. Ed. 2017, 56, 5170-5181.

(126) Chirik, P. J. Iron- and Cobalt-Catalyzed Alkene Hydrogenation: Catalysis with Both Redox-Active and Strong Field Ligands. Acc. Chem. Res. 2015, 48, 1687-1695.

(127) Lyaskovskyy, V.; De Bruin, B. Redox Non-Innocent Ligands: Versatile New Tools to Control Catalytic Reactions. ACS Catal. 2012, 2, 270-279.

(128) Harvey, J. N.; Poli, R. Computational Study of the SpinForbidden $\mathrm{H}_{2}$ Oxidative Addition to 16-Electron $\mathrm{Fe}(\mathrm{o})$ Complexes. Dalton Trans. 2003, 2, 4100-4106.

(129) Macgregor, S. A.; Eisenstein, O.; Whittlesey, M. K.; Perutz,

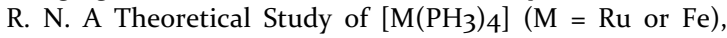
Models for the Highly Reactive d8 Intermediates $\left[\mathrm{M}(\mathrm{dmpe})_{2}\right]\left(\right.$ Dmpe $\left.=\mathrm{Me}_{2} \mathrm{PCH}_{2} \mathrm{CH}_{2} \mathrm{PMe}_{2}\right)$. Zero Activation Energies for Addition of $\mathrm{CO}$ and Oxidative Addition of $\mathrm{H}_{2} \neq$. J. Chem. Soc. Dalton Trans. 1998, 1, 291-300.

(130) Figgis, B. N.; Hitchman, M. A. Ligand Field Theory and Its Applications; Wiley-VCH, 2000.

(131) Poli, R.; Harvey, J. N. Spin Forbidden Chemical Reactions of Transition Metal Compounds. New Ideas and New Computational Challenges. Chem. Soc. Rev. 2oo3, 32, 1-8.

(132) Schröder, D.; Shaik, S.; Schwarz, H. Two-State Reactivity as a New Concept in Organometallic Chemistry. Acc. Chem. Res. 2000, 33, 139-145.

(133) Lomont, J. P.; Nguyen, S. C.; Harris, C. B. Ultrafast Infrared Studies of the Role of Spin States in Organometallic Reaction Dynamics. Acc. Chem. Res. 2014, 47, 1634-1642.

(134) Holland, P. L. Distinctive Reaction Pathways at Base Metals in High-Spin Organometallic Catalysts. Acc. Chem. Res. 2015, 48, 1696-1702.

(135) Shaik, S.; Chen, H.; Janardanan, D. Exchange-Enhanced Reactivity in Bond Activation by Metal-oxo Enzymes and Synthetic Reagents. Nat. Chem. 2011, 3, 19-27.

(136) Mukhopadhyay, T. K.; Rock, C. L.; Hong, M.; Ashley, D. C.; Groy, T. L.; Baik, M. H.; Trovitch, R. J. Mechanistic Investigation of Bis(imino)pyridine Manganese Catalyzed Carbonyl and Carboxylate Hydrosilylation. J. Am. Chem. Soc. 2017, 139, 4901-4915.

(137) Stieber, S. C. E.; Milsmann, C.; Hoyt, J. M.; Turner, Z. R.;
Finkelstein, K. D.; Wieghardt, K.; Debeer, S.; Chirik, P. J. Bis(imino)pyridine Iron Dinitrogen Compounds Revisited: Differences in Electronic Structure between Four- and FiveCoordinate Derivatives. Inorg. Chem. 2012, 51, 3770-3785.

(138) Ray, K.; Petrenko, T.; Wieghardt, K.; Neese, F. Joint Spectroscopic and Theoretical Investigations of Transition Metal Complexes Involving Non-Innocent Ligands. Dalton Trans. 2007, No. 16, 1552.

(139) Jørgensen, C. K. Differences between the Four Halide Ligands, and Discussion Remarks on Trigonal-Bipyramidal Complexes, on Oxidation States, and on Diagonal Elements of One-Electron Energy. Coord. Chem. Rev. 1966, 1, 164-178.

(140) Hindson, K. Cooperative \& Redox Non-Innocent Ligands in Directing Organometallic Reactivity. Eur. J. Inorg. Chem. 2012, No. 3, 340.

(141) Tondreau, A. M.; Milsmann, C.; Lobkovsky, E.; Chirik, P. J. Oxidation and Reduction of Bis(imino)pyridine Iron Dicarbonyl Complexes. Inorg. Chem. 2011, 5o, 9888-9895.

(142) Kaim, W. Manifestations of Noninnocent Ligand Behavior. Inorg. Chem. 2011, 50, 9752-9765.

(143) Chirik, P. J. Preface: Forum on Redox-Active Ligands. Inorg. Chem. 2011, 50, 9737-9740.

(144) Bouwkamp, M. W.; Bart, S. C.; HawrelakPresent address: Department, E. J.; Trovitch, R. J.; Lobkovsky, E.; Chirik, P. J. Square Planar Bis(imino)pyridine Iron Halide and Alkyl Complexes. Chem. Commun. 2005, No. 27, 3406.

(145) Kooistra, T. M.; Knijnenburg, Q.; Smits, J. M. M.; Horton, A. D.; Budzelaar, P. H. M.; Gal, A. W. Olefin Polymerization with [\{bis(imino)pyridyl\}CoIICl2]: Generation of the Active Species Involves CoI. Angew. Chemie 2001, 113, 4855-4858.

(146) Darmon, J. M.; Stieber, S. C. E.; Sylvester, K. T.; Fernández, I.; Lobkovsky, E.; Semproni, S. P.; Bill, E.; Wieghardt, K.; Debeer, S.; Chirik, P. J. Oxidative Addition of Carbon-Carbon Bonds with a Redox-Active Bis(imino)pyridine Iron Complex. J. Am. Chem. Soc. 2012, 134, 17125-17137.

(147) Atienza, C. C. H.; Milsmann, C.; Semproni, S. P.; Turner, Z. R.; Chirik, P. J. Reversible Carbon-Carbon Bond Formation Induced by Oxidation and Reduction at a Redox-Active Cobalt Complex. Inorg. Chem. 2o13, 52, 5403-5417.

(148) Trovitch, R. J.; Lobkovsky, E.; Chirik, P. J. Bis(imino)pyridine Iron Alkyls Containing $\beta$-Hydrogens: Synthesis, Evaluation of Kinetic Stability, and Decomposition Pathways Involving Chelate Participation. J. Am. Chem. Soc. 2oo8, 130, 1163111640 .

(149) Zhu, D.; Korobkov, I.; Budzelaar, P. H. M. Radical Mechanisms in the Reaction of Organic Halides with Diiminepyridine Cobalt Complexes. Organometallics 2012, 31, 3958-3971.

(150) Zhu, D.; Budzelaar, P. H. M. Binuclear Oxidative Addition of Aryl Halides. Organometallics 2010, 29, 5759-5761.

(151) Zhu, D.; Thapa, I.; Korobkov, I.; Gambarotta, S.; Budzelaar, P. H. M. Redox-Active Ligands and Organic Radical Chemistry. Inorg. Chem. 2011, 50, 9879-9887.

(152) Tondreau, A. M.; Stieber, S. C. E.; Milsmann, C.; Lobkovsky, E.; Weyhermüller, T.; Semproni, S. P.; Chirik, P. J. Oxidation and Reduction of Bis(imino)pyridine Iron Dinitrogen Complexes: Evidence for Formation of a Chelate Trianion. Inorg. Chem. 2013, 52, 635-646.

(153) Bart, S. C.; Chłopek, K.; Bill, E.; Bouwkamp, M. W.; Lobkovsky, E.; Neese, F.; Wieghardt, K.; Chirik, P. J. Electronic Structure of Bis(imino)pyridine Iron Dichloride, Monochloride, and Neutral Ligand Complexes: A Combined Structural, Spectroscopic, and Computational Study. J. Am. Chem. Soc. 2006, 128, 13901-13912.

(154) Bowman, A. C.; Milsmann, C.; Atienza, C. C. H.; Lobkovsky, E.; Wieghardt, K.; Chirik, P. J. Synthesis and Molecular and Electronic Structures of Reduced Bis(imino)pyridine Cobalt Dinitrogen Complexes: Ligand versus Metal Reduction. J. Am. Chem. Soc. 2010, 132, 1676-1684.

(155) Chirik, P. J. Electronic Structures of Reduced Manganese, Iron, and Cobalt Complexes Bearing Redox-Active 
Bis(imino)pyridine Pincer Ligands. In Pincer and PincerType Complexes; Wiley-VCH Verlag GmbH \& Co. KGaA: Weinheim, Germany, 2014; pp 189-212.

(156) Scott, J.; Vidyaratne, I.; Korobkov, I.; Gambarotta, S.; Budzelaar, P. H. M. Multiple Pathways for Dinitrogen Activation during the Reduction of an Fe Bis(iminepyridine) Complex. Inorg. Chem. 2008, 47, 896-911.

(157) Budzelaar, P. H. M.; de Bruin, B.; Gal, A. W.; Wieghardt, K.; van Lenthe, J. H. Metal-to-Ligand Electron Transfer in Diiminopyridine Complexes of Mn-Zn. A Theoretical Study. Inorg. Chem. 2oo1, 40, 4649-4655.

(158) Wile, B. M.; Trovitch, R. J.; Bart, S. C.; Tondreau, A. M.; Lobkovsky, E.; Milsmann, C.; Bill, E.; Wieghardt, K.; Chirik, P. J. Reduction Chemistry of Aryl- and Alkyl-Substituted Bis(imino)pyridine Iron Dihalide Compounds: Molecular and Electronic Structures of [(PDI $\left.)_{2} \mathrm{Fe}\right]$ Derivatives. Inorg. Chem. 2009, 48, 4190-4200.

(159) Russell, S. K.; Bowman, A. C.; Lobkovsky, E.; Wieghardt, K.; Chirik, P. J. Synthesis and Electronic Structure of Reduced Bis(imino)pyridine Manganese Compounds. Eur. J. Inorg. Chem. 2012, No. 3, 535-545.

(160) Milsmann, C.; Semproni, S. P.; Chirik, P. J. N-N Bond Cleavage of 1,2-Diarylhydrazines and $\mathrm{N}-\mathrm{H}$ Bond Formation via $\mathrm{H}$-Atom Transfer in Vanadium Complexes Supported by a Redox-Active Ligand. J. Am. Chem. Soc. 2014, 136, 1209912107.

(161) Hoyt, J. M.; Sylvester, K. T.; Semproni, S. P.; Chirik, P. J. Synthesis and Electronic Structure of Bis(imino)pyridine Iron Metallacyclic Intermediates in Iron-Catalyzed Cyclization Reactions. J. Am. Chem. Soc. 2013, 135, 48624877 .

(162) Steiman, T. J.; Uyeda, C. Reversible Substrate Activation and Catalysis at an Intact Metal-Metal Bond Using a RedoxActive Supporting Ligand. J. Am. Chem. Soc. 2015, 137, 61046110.

(163) van der Vlugt, J. I. Radical-Type Reactivity and Catalysis via Single-Electron Transfer to or from Redox-Active Ligands. Chem. Eur. J. 2018.

(164) Blackmore, K. J.; Ziller, J. W.; Heyduk, A. F. "Oxidative Addition" to a zirconium(IV) Redox-Active Ligand Complex. Inorg. Chem. 2005, 44, 5559-5561.

(165) Bouwkamp, M. W.; Bowman, A. C.; Lobkovsky, E.; Chirik, P. J. Iron-Catalyzed $[2 \pi+2 \pi]$ Cycloaddition of A, $\omega$-Dienes: The Importance of Redox-Active Supporting Ligands. J. Am. Chem. Soc. 2006, 128, 13340-13341.

(166) Chirik, P. J.; Wieghardt, K. Radical Ligands Confer Nobility on Base-Metal Catalysts. Science 2010, 327, 794-795.

(167) Zhu, D.; Thapa, I.; Korobkov, I.; Gambarotta, S.; Budzelaar, P. H. M. Redox-Active Ligands and Organic Radical Chemistry. Inorg. Chem. 2011, 50, 9879-9887.

(168) Dzik, W. I.; Zhang, X. P.; de Bruin, B. Redox Noninnocence of Carbene Ligands: Carbene Radicals in (Catalytic) C-C Bond Formation. Inorg. Chem. 2o11, 50, 9896-9903.

(169) Kraft, S. J.; Williams, U. J.; Daly, S. R.; J. Schelter, E.; Kozimor, S. A.; Boland, K. S.; Kikkawa, J. M.; Forrest, W. P.; Christensen, C. N.; Schwarz, D. E.; et al. Synthesis, Characterization, and Multielectron Reduction Chemistry of Uranium Supported by Redox-Active $\alpha$-Diimine Ligands. Inorg. Chem. 2011, 50, 9838-9848.

(170) Pérez, C. M.; Rodríguez-Delgado, A.; Palma, P.; Álvarez, E.; Gutiérrez-Puebla, E.; Cámpora, J. Neutral and Cationic alkylmanganese(II) Complexes Containing 2,6Bisiminopyridine Ligands. Chem. Eur. J. 2010, 16, 1383413842.

(171) Scott, J.; Gambarotta, S.; Korobkov, I.; Budzelaar, P. H. M. Metal versus Ligand Alkylation in the Reactivity of the (Bisiminopyridinato)Fe Catalyst. J. Am. Chem. Soc. 2005, 127, 13019-13029.

(172) Fernández, I.; Trovitch, R. J.; Lobkovsky, E.; Chirik, P. J. Synthesis of Bis(imino)pyridine Iron Di- and Monoalkyl Complexes: Stability Differences between $\mathrm{FeCH}_{2}$ SiMe 3 and
FeCH 2 CMe 3 Derivatives. Organometallics 2008, 27, 109118.

(173) Budzelaar, P. H. M. Radical Chemistry of Iminepyridine Ligands. Eur. J. Inorg. Chem. 2012, No. 3, 530-534.

(174) Pramanick, R.; Bhattacharjee, R.; Sengupta, D.; Datta, A.; Goswami, S. An Azoaromatic Ligand as Four Electron Four Proton Reservoir: Catalytic Dehydrogenation of Alcohols by Its Zinc(II) Complex. Inorg. Chem. 2018, 57, 6816-6824.

(175) Sengupta, D.; Bhattacharjee, R.; Pramanick, R.; Rath, S. P.; Saha Chowdhury, N.; Datta, A.; Goswami, S. Exclusively Ligand-Mediated Catalytic Dehydrogenation of Alcohols. Inorg. Chem. 2016, 55, 9602-9610.

(176) Knijnenburg, Q.; Gambarotta, S.; Budzelaar, P. H. M. LigandCentred Reactivity in Diiminepyridine Complexes. Dalton Trans. 2006, No. 46, 5442.

(177) Enright, D.; Gambarotta, S.; Yap, G. P. A.; Budzelaar, P. H. $M$. The Ability of the A, $\alpha^{\prime}$-Diiminopyridine Ligand System to Accept Negative Charge: Isolation of Paramagnetic and Diamagnetic Trianions. Angew. Chem. Int. Ed. 2002, 41, 3873-3876.

(178) Bart, S. C.; Lobkovsky, E.; Bill, E.; Wieghardt, K.; Chirik, P. J. Neutral-Ligand Complexes of Bis(imino)pyridine Iron: Synthesis, Structure, and Spectroscopy. Inorg. Chem. 2007, 46, 7055-7063.

(179) Bowman, A. C.; Milsmann, C.; Bill, E.; Turner, Z. R.; Lobkovsky, E.; Debeer, S.; Wieghardt, K.; Chirik, P. J. Synthesis and Electronic Structure Determination of NAlkyl-Substituted Bis(imino)pyridine Iron Imides Exhibiting Spin Crossover Behavior. J. Am. Chem. Soc. 2011, 133, 1735317369 .

(180) Chakraborty, S.; Brennessel, W. W.; Jones, W. D. A Molecular Iron Catalyst for the Acceptorless Dehydrogenation and Hydrogenation of N-Heterocycles. J. Am. Chem. Soc. 2014, 136, 8564-8567.

(181) Bielinski, E. A.; Lagaditis, P. O.; Zhang, Y.; Mercado, B. Q.; Würtele, C.; Bernskoetter, W. H.; Hazari, N.; Schneider, S. Lewis Acid-Assisted Formic Acid Dehydrogenation Using a Pincer-Supported Iron Catalyst. J. Am. Chem. Soc. 2014, 136, 10234-10237.

(182) Chakraborty, S.; Dai, H.; Bhattacharya, P.; Fairweather, N. T.; Gibson, M. S.; Krause, J. A.; Guan, H. Iron-Based Catalysts for the Hydrogenation of Esters to Alcohols. J. Am. Chem. Soc. 2014, 136, 7869-7872.

(183) Tondreau, A. M.; Boncella, J. M. 1,2-Addition of Formic or Oxalic Acid to- $\mathrm{N}\left\{\mathrm{CH}_{2} \mathrm{CH}_{2}\left(\mathrm{P}^{i} \mathrm{Pr}_{2}\right)\right\}_{2}$-Supported $\mathrm{Mn}(\mathrm{I})$ Dicarbonyl Complexes and the Manganese-Mediated Decomposition of Formic Acid. Organometallics 2016, 35, 2049-2052.

(184) Nguyen, D. H.; Trivelli, X.; Capet, F.; Paul, J.-F.; Dumeignil, F.; Gauvin, R. M. Manganese Pincer Complexes for the BaseFree, Acceptorless Dehydrogenative Coupling of Alcohols to Esters: Development, Scope, and Understanding. ACS Catal. 2017, 7, 2022-2032.

(185) Schneck, F.; Assmann, M.; Balmer, M.; Harms, K.; Langer, R. Selective Hydrogenation of Amides to Amines and Alcohols Catalyzed by Improved Iron Pincer Complexes. Organometallics 2016, 35, 1931-1943.

(186) Friedrich, A.; Drees, M.; Schneider, S. Ruthenium-Catalyzed Dimethylamineborane Dehydrogenation: Stepwise MetalCentered Dehydrocyclization. Chem. Eur. J. 2009, 15, 1033910342.

(187) Marziale, A. N.; Friedrich, A.; Klopsch, I.; Drees, M.; Celinski, V. R.; Schmedt auf der Günne, J.; Schneider, S. The Mechanism of Borane-Amine Dehydrocoupling with Bifunctional Ruthenium Catalysts. J. Am. Chem. Soc. 2013, 135, 13342-13355.

(188) Askevold, B.; Friedrich, A.; Buchner, M. R.; Lewall, B.; Filippou, A. C.; Herdtweck, E.; Schneider, S. Reactivity of iridium(I) PNP Amido Complexes toward Protonation and Oxidation. J. Organomet. Chem. 2013, 744, 35-40.

(189) Bordwell, F. G.; Drucker, G. E.; Fried, H. E. Acidities of 
Carbon and Nitrogen Acids: The Aromaticity of the Cyclopentadienyl Anion. J. Org. Chem. 1981, 46, 632-635.

(190) Crampton, M. R.; Robotham, I. A. Acidities of Some Substituted Ammonium Ions in Dimethyl Sulfoxide†. J. Chem. Res. 1997, o, 22-23.

(191) Abdur-Rashid, K.; Fong, T. P.; Greaves, B.; Gusev, D. G.; Hinman, J. G.; Landau, S. E.; Lough, A. J.; Morris, R. H. An Acidity Scale for Phosphorus-Containing Compounds Including Metal Hydrides and Dihydrogen Complexes in THF: Toward the Unification of Acidity Scales. J. Am. Chem. Soc. 2000, 122, 9155-9171.

(192) Smith, N. E.; Bernskoetter, W. H.; Hazari, N.; Mercado, B. Q. Synthesis and Catalytic Activity of PNP-Supported Iron Complexes with Ancillary Isonitrile Ligands. Organometallics 2017, 36, 3995-4004.

(193) Wei, Z.; Junge, K.; Beller, M.; Jiao, H. Exploring the Activities of Vanadium, Niobium, and Tantalum PNP Pincer Complexes in the Hydrogenation of Phenyl-Substituted $\mathrm{C} \equiv \mathrm{N}, \mathrm{C}=\mathrm{N}, \mathrm{C} \equiv \mathrm{C}, \mathrm{C}=\mathrm{C}$, and $\mathrm{C}=\mathrm{O}$ Functional Groups. Comptes Rendus Chim. 2018, 21, 303-309.

(194) Wei, Z.; Junge, K.; Beller, M.; Jiao, H. Hydrogenation of Phenyl-Substituted $\mathrm{C} \equiv \mathrm{N}, \quad \mathrm{C}=\mathrm{N}, \quad \mathrm{C} \equiv \mathrm{C}, \quad \mathrm{C}=\mathrm{C}$ and $\mathrm{C}=\mathrm{O}$ Functional Groups by $\mathrm{Cr}$, Mo and W PNP Pincer Complexes - a DFT Study. Catal. Sci. Technol. 2017, 7, 2298-2307.

(195) Zhang, G.; Scott, B. L.; Hanson, S. K. Mild and Homogeneous Cobalt-Catalyzed Hydrogenation of $\mathrm{C}=\mathrm{C}, \mathrm{C}=\mathrm{O}$, and $\mathrm{C}=\mathrm{N}$ Bonds. Angew. Chem. Int. Ed. 2012, 51, 12102-12106.

(196) Xu, R.; Chakraborty, S.; Bellows, S. M.; Yuan, H.; Cundari, T. R.; Jones, W. D. Iron-Catalyzed Homogeneous Hydrogenation of Alkenes under Mild Conditions by a Stepwise, Bifunctional Mechanism. ACS Catal. 2016, 6, 21272135.

(197) Junge, K.; Wendt, B.; Cingolani, A.; Spannenberg, A.; Wei, Z.; Jiao, H.; Beller, M. Cobalt Pincer Complexes for Catalytic Reduction of Carboxylic Acid Esters. Chem. Eur. J. 2018, 24, 1046-1052.

(198) Yang, X. Hydrogenation of Carbon Dioxide Catalyzed by PNP Pincer Iridium, Iron, and Cobalt Complexes: A Computational Design of Base Metal Catalysts. ACS Catal. 2011, 1, 849-854.

(199) Rozenel, S. S.; Padilla, R.; Arnold, J. Chemistry of Reduced Monomeric and Dimeric Cobalt Complexes Supported by a PNP Pincer Ligand. Inorg. Chem. 2013, 52, 11544-1155o.

(20o) Rozenel, S. S.; Padilla, R.; Camp, C.; Arnold, J. Unusual Activation of $\mathrm{H}_{2}$ by Reduced Cobalt Complexes Supported by a PNP Pincer Ligand. Chem. Commun. 2014, 50, 2612.

(201) Vasudevan, K. V.; Scott, B. L.; Hanson, S. K. Alkene Hydrogenation Catalyzed by Nickel Hydride Complexes of an Aliphatic PNP Pincer Ligand. Eur. J. Inorg. Chem. 2012, 2012, 4898-4906.

(202) Adhikari, D.; Mossin, S.; Basuli, F.; Huffman, J. C.; Szilagyi, R. K.; Meyer, K.; Mindiola, D. J. Structural, Spectroscopic, and Theoretical Elucidation of a Redox-Active Pincer-Type Ancillary Applied in Catalysis. J. Am. Chem. Soc. 20o8, 130, 3676-3682.

(203) Schneck, F.; Finger, M.; Tromp, M.; Schneider, S. Chemical Non-Innocence of an Aliphatic PNP Pincer Ligand. Chem. Eur. J. 2017, 23, 33-37.

(204) Lagaditis, P. O.; Schluschaß, B.; Demeshko, S.; Würtele, C.; Schneider, S. Square-Planar Cobalt(III) Pincer Complex. Inorg. Chem. 2016, 55, 4529-4536.

(205) Rosenkoetter, K. E.; Wojnar, M. K.; Charette, B. J.; Ziller, J. W.; Heyduk, A. F. Hydrogen-Atom Noninnocence of a Tridentate [SNS] Pincer Ligand. Inorg. Chem. 2018, 57, 97289737.

(206) Schneck, F.; Ahrens, J.; Finger, M.; Stückl, A. C.; Würtele, C.; Schwarzer, D.; Schneider, S. The Elusive Abnormal $\mathrm{CO}_{2}$ Insertion Enabled by Metal-Ligand Cooperative Photochemical Selectivity Inversion. Nat. Commun. 2018, 9, 1161.

(207) Schneck, F.; Schendzielorz, F.; Hatami, N.; Finger, M.;
Würtele, C.; Schneider, S. Photochemically Driven Reverse Water-Gas Shift at Ambient Conditions Mediated by a Nickel Pincer Complex. Angew. Chem. Int. Ed. 2018, 10.

(208) Sauer, D. C.; Kruck, M.; Wadepohl, H.; Enders, M.; Gade, L. H. Spin Density Distribution in iron(II) and cobalt(II) Alkyl Complexes Containing 1,3-Bis(2-Pyridylimino)isoindolate Ligands. Organometallics 2013, 32, 885-892.

(209) Blasius, C. K.; Vasilenko, V.; Gade, L. H. Ultrafast IronCatalyzed Reduction of Functionalized Ketones: Highly Enantioselective Synthesis of Halohydrines, Oxaheterocycles, and Aminoalcohols. Angew. Chem. Int. Ed. 2018, 57, 10231-10235.

(210) Bleith, T.; Wadepohl, H.; Gade, L. H. Iron Achieves Noble Metal Reactivity and Selectivity: Highly Reactive and Enantioselective Iron Complexes as Catalysts in the Hydrosilylation of Ketones. J. Am. Chem. Soc. 2015, 137, 2456-2459.

(211) Schiwek, C. H.; Vasilenko, V.; Wadepohl, H.; Gade, L. H. The Open D-Shell Enforces the Active Space in 3d Metal Catalysis: Highly Enantioselective chromium(II) Pincer Catalysed Hydrosilylation of Ketones. Chem. Commun. 2o18, 54, 9139-9142.

(212) Vasilenko, V.; Blasius, C. K.; Wadepohl, H.; Gade, L. H. Mechanism-Based Enantiodivergence in Manganese Reduction Catalysis: A Chiral Pincer Complex for the Highly Enantioselective Hydroboration of Ketones. Angew. Chem. Int. Ed. 2017, 56, 8393-8397.

(213) Vasilenko, V.; Blasius, C. K.; Gade, L. H. One-Pot Sequential Kinetic Profiling of a Highly Reactive Manganese Catalyst for Ketone Hydroboration: Leveraging $\sigma$-Bond Metathesis via Alkoxide Exchange Steps. J. Am. Chem. Soc. 2018, 140, 92449254 .

(214) Wenz, J.; Vasilenko, V.; Kochan, A.; Wadepohl, H.; Gade, L. $\mathrm{H}$. Coordination Chemistry of the PdmBOX Pincer Ligand: Reactivity at the Metal and the Ligand. Eur. J. Inorg. Chem. 2017, 2017, 5545-5556.

(215) Deng, Q. H.; Wadepohl, H.; Gade, L. H. The Synthesis of a New Class of Chiral Pincer Ligands and Their Applications in Enantioselective Catalytic Fluorinations and the NozakiHiyama-Kishi Reaction. Chem. Eur. J. 2011, 17, 14922-14928.

(216) Altwicker, E. R. The Chemistry of Stable Phenoxy Radicals. Chem. Rev. 1967, 67, 475-531.

(217) Broere, D. L. J.; Plessius, R.; van der Vlugt, J. I. New Avenues for Ligand-Mediated Processes - Expanding Metal Reactivity by the Use of Redox-Active Catechol, O-Aminophenol and O-Phenylenediamine Ligands. Chem. Soc. Rev. 2015, 44, 6886-6915.

(218) Broere, D. L. J.; De Bruin, B.; Reek, J. N. H.; Lutz, M.; Dechert, S.; Van Der Vlugt, J. I. Intramolecular Redox-Active Ligandto-Substrate Single-Electron Transfer: Radical Reactivity with a palladium(II) Complex. J. Am. Chem. Soc. 2014, 136, 11574-11577.

(219) Bagh, B.; Broere, D. L. J.; Sinha, V.; Kuijpers, P. F.; Van Leest, N. P.; De Bruin, B.; Demeshko, S.; Siegler, M. A.; Van Der Vlugt, J. I. Catalytic Synthesis of N-Heterocycles via Direct $\mathrm{C}\left(\mathrm{sp}_{3}\right)-\mathrm{H}$ Amination Using an Air-Stable Iron(III) Species with a Redox-Active Ligand. J. Am. Chem. Soc. 2017, 139, 51175124 .

(220) Broere, D. L. J.; Metz, L. L.; De Bruin, B.; Reek, J. N. H.; Siegler, M. A.; Van Der Vlugt, J. I. Redox-Active LigandInduced Homolytic Bond Activation. Angew. Chem. Int. Ed. 2015, 54, 1516-1520.

(221) Harris, C. F.; Bayless, M. B.; Van Leest, N. P.; Bruch, Q. J.; Livesay, B. N.; Bacsa, J.; Hardcastle, K. I.; Shores, M. P.; De Bruin, B.; Soper, J. D. Redox-Active Bis(phenolate) NHeterocyclic Carbene [OCO] Pincer Ligands Support Cobalt Electron Transfer Series Spanning Four Oxidation States. Inorg. Chem. 2017, 56, 12421-12435.

(222) Sikari, R.; Sinha, S.; Jash, U.; Das, S.; Brandao, P.; De Bruin, B.; Paul, N. D. Deprotonation Induced Ligand Oxidation in a NiIIComplex of a Redox Noninnocent N1-(2- 
Aminophenyl)benzene-1,2-Diamine and Its Use in Catalytic Alcohol Oxidation. Inorg. Chem. 2016, 55, 6114-6123.

(223) Chang, M.-C.; McNeece, A. J.; Hill, E. A.; Filatov, A. S.; Anderson, J. S. Ligand-Based Storage of Protons and Electrons in Dihydrazonopyrrole Complexes of Nickel. Chem. Eur. J. 2018, 24, 8001-80o8.

(224) Dahlhoff, W. V.; Nelson, S. M. Studies on the Magnetic Cross-over in Five-Co-Ordinate Complexes of iron(II), cobalt(II), and nickel(II). Part II. J. Chem. Soc. A Inorganic, Phys. Theor. 1971, No. 2184, 2184.

(225) Zell, T.; Milstein, D. Hydrogenation and Dehydrogenation Iron Pincer Catalysts Capable of Metal-Ligand Cooperation by Aromatization/Dearomatization. Acc. Chem. Res. 2015, 48, 1979-1994.

(226) Fraser, R. R.; Bresse, M.; Mansour, T. S. pK a Measurements in Tetrahydrofuran. J. Chem. Soc. Chem. Commun. 1983, No. 11, 620.

(227) Simler, T.; Frison, G.; Braunstein, P.; Danopoulos, A. A. Direct Synthesis of Doubly Deprotonated, Dearomatised Lutidine PNP $\mathrm{Cr}$ and $\mathrm{Zr}$ Pincer Complexes Based on Isolated $\mathrm{K}$ and Li Ligand Transfer Reagents. Dalton Trans. 2016, 45, 2800-2804.

(228) Simler, T.; Braunstein, P.; Danopoulos, A. A. Chromium(II) Pincer Complexes with Dearomatized PNP and PNC Ligands: A Comparative Study of Their Catalytic Ethylene Oligomerization Activity. Organometallics 2016, 35, 40444049.

(229) Vogt, M.; Nerush, A.; Iron, M. A.; Leitus, G.; Diskin-Posner, Y.; Shimon, L. J. W.; Ben-David, Y.; Milstein, D. Activation of Nitriles by Metal Ligand Cooperation. Reversible Formation of Ketimido- and Enamido-Rhenium PNP Pincer Complexes and Relevance to Catalytic Design. J. Am. Chem. Soc. 2o13, 135, 17004-17018.

(230) Zhang, J.; Leitus, G.; Ben-David, Y.; Milstein, D. Efficient Homogeneous Catalytic Hydrogenation of Esters to Alcohols. Angew. Chem. Int. Ed. 2006, 45, 1113-1115.

(231) Fogler, E.; Iron, M. A.; Zhang, J.; Ben-David, Y.; Diskin-Posner, Y.; Leitus, G.; Shimon, L. J. W.; Milstein, D. $\mathrm{Ru}(\mathrm{o})$ and $\mathrm{Ru}(\mathrm{II})$ Nitrosyl Pincer Complexes: Structure, Reactivity, and Catalytic Activity. Inorg. Chem. 2013, 52, 11469-11479.

(232) Fogler, E.; Efremenko, I.; Gargir, M.; Leitus, G.; DiskinPosner, Y.; Ben-David, Y.; Martin, J. M. L.; Milstein, D. New Ruthenium Nitrosyl Pincer Complexes Bearing an $\mathrm{O}_{2}$ Ligand. Mono-Oxygen Transfer. Inorg. Chem. 2015, 54, 22532263.

(233) Anaby, A.; Feller, M.; Ben-David, Y.; Leitus, G.; DiskinPosner, Y.; Shimon, L. J. W.; Milstein, D. Bottom-Up Construction of a $\mathrm{CO} 2$-Based Cycle for the Photocarbonylation of Benzene, Promoted by a Rhodium(I) Pincer Complex. J. Am. Chem. Soc. 2016, 138, 9941-9950.

(234) Ben-Ari, E.; Leitus, G.; Shimon, L. J. W.; Milstein, D. Metal-Ligand Cooperation in $\mathrm{C}-\mathrm{H}$ and $\mathrm{H} 2$ Activation by an Electron-Rich PNP Ir(I) System: Facile Ligand Dearomatization-Aromatization as Key Steps. J. Am. Chem. Soc. 2006, 128, 15390-15391.

(235) Schwartsburd, L.; Iron, M. A.; Konstantinovski, L.; DiskinPosner, Y.; Leitus, G.; Shimon, L. J. W.; Milstein, D. Synthesis and Reactivity of an Iridium(I) Acetonyl PNP Complex. Experimental and Computational Study of Metal-Ligand Cooperation in $\mathrm{H}-\mathrm{H}$ and $\mathrm{C}-\mathrm{H}$ Bond Activation via Reversible Ligand Dearomatization. Organometallics 2010, 29, 3817-3827.

(236) Vogt, M.; Rivada-Wheelaghan, O.; Iron, M. A.; Leitus, G.; Diskin-Posner, Y.; Shimon, L. J. W.; Ben-David, Y.; Milstein, D. Anionic Nickel(II) Complexes with Doubly Deprotonated PNP Pincer-Type Ligands and Their Reactivity toward CO 2. Organometallics 2013, 32, 300-308.

(237) Sacco, A.; Vasapollo, G.; Nobile, C. F.; Piergiovanni, A.; Pellinghelli, M. A.; Lanfranchi, M. Syntheses and Structures of 2-Diphenylphosphinomethylenide-6-
Diphenylphosphinomethylenepyridine Complexes of palladium(II) and platinum(II); Crystal Structures of $\left[\mathrm{PtCl}_{2-}\right.$ (CHPPH2)-6-( $\left.\mathrm{CH}_{2} \mathrm{PPh}_{2}\right)$ pyridine] and [Pd(COOMe $)_{2}$ (CHPPh2)-6-( $\left.\mathrm{CH}_{2} \mathrm{PPh}_{2}\right)$ pyridine]. J. Organomet. Chem. 1988, 356, 397-409.

(238) Feller, M.; Ben-Ari, E.; Iron, M. A.; Diskin-Posner, Y.; Leitus, G.; Shimon, L. J. W.; Konstantinovski, L.; Milstein, D. Cationic, Neutral, and Anionic PNP Pd II and Pt II Complexes: Dearomatization by Deprotonation and DoubleDeprotonation of Pincer Systems. Inorg. Chem. 2010, 49, 1615-1625.

(239) Taguchi, H.; Chang, Y.-H.; Takeuchi, K.; Ozawa, F. Catalytic Synthesis of an Unsymmetrical PNP-Pincer-Type Phosphaalkene Ligand. Organometallics 2015, 34, 1589-1596.

(240) Kosanovich, A. J.; Komatsu, C. H.; Bhuvanesh, N.; Pérez, L. M.; Ozerov, O. V. Dearomatization of the PCP Pincer Ligand in a Re V Oxo Complex. Chem. Eur. J. 2018.

(241) Langer, R.; Diskin-Posner, Y.; Leitus, G.; Shimon, L. J. W.; Ben-David, Y.; Milstein, D. Low-Pressure Hydrogenation of Carbon Dioxide Catalyzed by an Iron Pincer Complex Exhibiting Noble Metal Activity. Angew. Chem. Int. Ed. 2011, 50, 9948-9952.

(242) Khaskin, E.; Diskin-Posner, Y.; Weiner, L.; Leitus, G.; Milstein, D. Formal Loss of an $\mathrm{H}$ Radical by a Cobalt Complex via Metal-ligand Cooperation. Chem. Commun. 2013, 49, 2771.

(243) Semproni, S. P.; Milsmann, C.; Chirik, P. J. Four-Coordinate Cobalt Pincer Complexes: Electronic Structure Studies and Ligand Modification by Homolytic and Heterolytic Pathways. J. Am. Chem. Soc. 2014, 136, 9211-9224.

(244) Semproni, S. P.; Hojilla Atienza, C. C.; Chirik, P. J. Oxidative Addition and $\mathrm{C}-\mathrm{H}$ Activation Chemistry with a PNP PincerLigated Cobalt Complex. Chem. Sci. 2014, 5, 1956-1960.

(245) van der Vlugt, J. I.; Lutz, M.; Pidko, E. A.; Vogt, D.; Spek, A. L. Cationic and Neutral Ni II Complexes Containing a NonInnocent PNP Ligand: Formation of Alkyl and Thiolate Species. Dalton Trans. 2009, No. 6, 1016-1023.

(246) van der Vlugt, J. I.; Pidko, E. a; Vogt, D.; Lutz, M.; Spek, A. L.; Meetsma, A. T-Shaped Cationic Cu I Complexes with Hemilabile PNP-Type Ligands. Inorg. Chem. 2oo8, 47, 44424444 .

(247) Van Der Vlugt, J. I.; Pidko, E. A.; Vogt, D.; Lutz, M.; Spek, A. L. CuIcomplexes with a Noninnocent PNP Ligand: Selective Dearomatization and Electrophilic Addition Reactivity. Inorg. Chem. 2009, 48, 7513-7515.

(248) De Boer, S. Y.; Gloaguen, Y.; Lutz, M.; Van Der Vlugt, J. I. Culclick Catalysis with Cooperative Noninnocent Pyridylphosphine Ligands. Inorg. Chim. Acta 2012, 380, 336342.

(249) Alzamly, A.; Gambarotta, S.; Korobkov, I. Polymer-Free Ethylene Oligomerization Using a Pyridine-Based Pincer PNP-Type of Ligand. Organometallics 2013, 32, 7204-7212.

(250) Deibl, N.; Kempe, R. Manganese-Catalyzed Multicomponent Synthesis of Pyrimidines from Alcohols and Amidines. Angew. Chem. Int. Ed. 2017, 56, 1663-1666.

(251) Bichler, B.; Holzhacker, C.; Stöger, B.; Puchberger, M.; Veiros, L. F.; Kirchner, K. Heterolytic Cleavage of Dihydrogen by an Iron(II) PNP Pincer Complex via MetalLigand Cooperation. Organometallics 2013, 32, 4114-4121.

(252) Mastalir, M.; Stöger, B.; Pittenauer, E.; Allmaier, G.; Kirchner, K. Air-Stable Triazine-Based Ni(II) PNP Pincer Complexes As Catalysts for the Suzuki-Miyaura CrossCoupling. Org. Lett. 2016, 18, 3186-3189.

(253) Mastalir, M.; Kirchner, K. A Triazine-Based Ni(II) PNP Pincer Complex as Catalyst for Kumada-Corriu and Negishi Cross-Coupling Reactions. Monatsh. Chem. 2017, 148, 105109.

(254) Kumar, L. M.; Ansari, R. M.; Bhat, B. R. Catalytic Activity of $\mathrm{Fe}(\mathrm{II})$ and $\mathrm{Cu}(\mathrm{II})$ PNP Pincer Complexes for Suzuki Coupling Reaction. Appl. Organomet. Chem. 2018, 32, e4054.

(255) Mastalir, M.; Glatz, M.; Stöger, B.; Weil, M.; Pittenauer, E.; 
Allmaier, G.; Kirchner, K. Synthesis, Characterization and Reactivity of Vanadium, Chromium, and Manganese PNP Pincer Complexes. Inorg. Chim. Acta 2017, 455, 707-714.

(256) Mastalir, M.; de Aguiar, S. R. M. M.; Glatz, M.; Stöger, B.; Kirchner, K. A Convenient Solvothermal Synthesis of Group 6 PNP Pincer Tricarbonyl Complexes. Organometallics 2016, 35, 229-232.

(257) Alzamly, A.; Gambarotta, S.; Korobkov, I. Reactivity with Alkylaluminum of a Chromium Complex of a PyridineContaining PNP Ligand: Redox N-P Bond Cleavage. Organometallics 2014, 33, 1602-1607.

(258) Gibson, V. C.; Humphries, M. J.; Tellmann, K. P.; Wass, D. F.; White, A. J. P.; Williams, D. J.; Britovsek, G. J. P.; Gibson, V. C.; Kimberley, B. S.; Solan, G. A.; et al. The Nature of the Active Species in Bis(imino)pyridyl Cobalt Ethylene Polymerisation Catalysts. Chem. Commun. 2001, 120, 22522253.

(259) Small, B. L.; Brookhart, M.; Bennett, A. M. a. Highly Active Iron and Cobalt Catalysts for the Polymerization of Ethylene. J. Am. Chem. Soc. 1998, 120, 4049-4050.

(26o) Britovsek, G. J. P.; Bruce, M.; Gibson, V. C.; Kimberley, B. S.; Maddox, P. J.; Mastroianni, S.; McTavish, S. J.; Redshaw, C.; Solan, G. A.; Strömberg, S.; et al. Iron and Cobalt Ethylene Polymerization Catalysts Bearing 2,6-Bis(Imino)Pyridyl Ligands: Synthesis, Structures, and Polymerization Studies. J. Am. Chem. Soc. 1999, 121, 8728-8740.

(261) Cámpora, J.; Naz, A. M.; Palma, P.; Álvarez, E.; Reyes, M. L. 2,6-Diiminopyridine Iron(II) Dialkyl Complexes. Interaction with Aluminum Alkyls and Ethylene Polymerization Catalysis. Organometallics 2005, 24, 4878-4881.

(262) Bouwkamp, M. W.; Lobkovsky, E.; Chirik, P. J. Bis (Imino)pyridine Iron(II) Alkyl Cations for Olefin Polymerization. J. Am. Chem. Soc. Commun. 2005, 316, 966o9661.

(263) Cámpora, J.; Naz, A. M.; Palma, P.; Rodríguez-Delgado, A.; Álvarez, E.; Tritto, I.; Boggioni, L. Iron and Cobalt Complexes of 4-Alkyl-2,6-Diiminopyridine Ligands: Synthesis and Ethylene Polymerization Catalysis. Eur. J. Inorg. Chem. 20o8, 2, 1871-1879.

(264) Yu, J.; Liu, H.; Zhang, W.; Hao, X.; Sun, W.-H. Access to Highly Active and Thermally Stable Iron Procatalysts Using Bulky 2-[1-(2,6-Dibenzhydryl-4-Methylphenylimino)ethyl]6-[1-(Arylimino)ethyl]pyridine Ligands. Chem. Commun. 2011, 47, 3257.

(265) Yu, J.; Huang, W.; Wang, L.; Redshaw, C.; Sun, W.-H. 2-[1(2,6-Dibenzhydryl-4-Methylphenylimino)ethyl]-6-[1(Arylimino)ethyl]pyridylcobalt(ii) Dichlorides: Synthesis, Characterization and Ethylene Polymerization Behavior. Dalton Trans. 2011, 40, 10209.

(266) Xing, Q.; Zhao, T.; Qiao, Y.; Wang, L.; Redshaw, C.; Sun, W.H. Synthesis, Characterization and Ethylene Polymerization Behavior of Binuclear Iron Complexes Bearing N,N'-bis(1-(6(1-(Arylimino)ethyl) Pyridin-2-Yl)ethylidene)benzidines. RSC Adv. 2013, 3, 26184.

(267) Tondreau, A. M.; Milsmann, C.; Patrick, A. D.; Hoyt, H. M.; Lobkovsky, E.; Wieghardt, K.; Chirik, P. J. Synthesis and Electronic Structure of Cationic, Neutral, and Anionic Bis(imino)pyridine Iron Alkyl Complexes: Evaluation of Redox Activity in Single-Component Ethylene Polymerization Catalysts. J. Am. Chem. Soc. 2010, 132, 1504615059.

(268) Bryliakov, K. P.; Talsi, E. P.; Semikolenova, N. V; Zakharov, V. A. Formation and Nature of the Active Sites in Bis(imino)pyridine Iron-Based Polymerization Catalysts. Organometallics 2009, 28, 3225-3232.

(269) Appukuttan, V. K.; Liu, Y.; Son, B. C.; Ha, C.-S.; Suh, H.; Kim, I. Iron and Cobalt Complexes of 2,3,7,8-Tetrahydroacridine4,5 $(1 \mathrm{H}, 6 \mathrm{H})$-Diimine Sterically Modulated by Substituted Aryl Rings for the Selective Oligomerization to Polymerization of Ethylene. Organometallics 2011, 30, 22852294.
(270) Bianchini, C.; Mantovani, G.; Meli, A.; Migliacci, F.; Zanobini, F.; Laschi, F.; Sommazzi, A. Oligomerisation of Ethylene to Linear $\alpha$-Olefins by New Cs- and C1-Symmetric [2,6-Bis(imino)pyridyl]iron and -Cobalt Dichloride Complexes. Eur. J. Inorg. Chem. 2003, 1620-1631.

(271) Margl, P.; Deng, L.; Ziegler, T. Cobalt(II) Imino Pyridine Assisted Ethylene Polymerization: A QuantumMechanical/Molecular-Mechanical Density Functional Theory Investigation. Organometallics 1999, 18, 5701-5708.

(272) Gibson, V. C.; Tellmann, K. P.; Humphries, M. J.; Wass, D. F. Bis (Imino) Pyridine Cobalt Alkyl Complexes and Their Reactivity towards Ethylene: A Model System for BetaHydrogen Chain Transfer. Chem. Commun. 2oo2, 2316-2317.

(273) Xing, Q.; Zhao, T.; Du, S.; Yang, W.; Liang, T.; Redshaw, C.; Sun, W. Biphenyl-Bridged 6 - (1-Aryliminoethyl)-2Iminopyridylcobalt Complexes: Synthesis, Characterization, and Ethylene Polymerization Behavior. Organometallics 2014, 33, 1382-1388.

(274) Antonov, A. A.; Semikolenova, N. V.; Zakharov, V. A.; Zhang, W.; Wang, Y.; Sun, W. H.; Talsi, E. P.; Bryliakov, K. P. Vinyl Polymerization of Norbornene on Nickel Complexes with Bis(imino)pyridine Ligands Containing ElectronWithdrawing Groups. Organometallics 2012, 31, 1143-1149.

(275) Esteruelas, M. A.; López, A. M.; Méndez, L.; Oliván, M.; Oñate, E. Preparation, Structure, and Ethylene Polymerization Behavior of Bis(imino)pyridyl Chromium(III) Complexes. Organometallics 2003, 22, 395406.

(276) Vidyaratne, I.; Scott, J.; Gambarotta, S.; Duchateau, R. Reactivity of Chromium Complexes of a Bis(imino)pyridine Ligand: Highly Active Ethylene Polymerization Catalysts Carrying the Metal in a Formally Low Oxidation State. Organometallics 2007, 26, 3201-3211.

(277) Reardon, D.; Conan, F.; Gambarotta, S.; Yap, G.; Wang, Q. Life and Death of an Active Ethylene Polymerization Catalyst. Ligand Involvement in Catalyst Activation and Deactivation. Isolation and Characterization of Two Unprecedented Neutral and Anionic vanadium(I) Alkyls. J. Am. Chem. Soc. 1999, 121, 9318-9325.

(278) Mahmood, Q.; Guo, J.; Zhang, W.; Ma, Y.; Liang, T.; Sun, W.H. Concurrently Improving the Thermal Stability and Activity of Ferrous Precatalysts for the Production of Saturated/Unsaturated Polyethylene. Organometallics 2018, 37, 957-970.

(279) Schaefer, B. A.; Margulieux, G. W.; Tiedemann, M. A.; Small, B. L.; Chirik, P. J. Synthesis and Electronic Structure of Iron Borate Betaine Complexes as a Route to Single-Component Iron Ethylene Oligomerization and Polymerization Catalysts. Organometallics 2015, 34, 5615-5623.

(28o) Ortuño, M. A.; Dereli, B.; Chiaie, K. R. D.; Biernesser, A. B.; Qi, M.; Byers, J. A.; Cramer, C. J. The Role of Alkoxide Initiator, Spin State, and Oxidation State in Ring-Opening Polymerization of $\varepsilon$-Caprolactone Catalyzed by Iron Bis(imino)pyridine Complexes. Inorg. Chem. 2018, 57, 20642071.

(281) Cartes, M. Á.; Rodríguez-Delgado, A.; Palma, P.; Álvarez, E.; Cámpora, J. Sequential Reduction and Alkyl Exchange Reactions of Bis(imino)pyridine Dialkyliron(II) with Trimethylaluminum. Organometallics 2014, 33, 1834-1839.

(282) Bianchini, C.; Giambastiani, G.; Luconi, L.; Meli, A. Olefin Oligomerization, Homopolymerization and Copolymerization by Late Transition Metals Supported by (Imino)pyridine Ligands. Coord. Chem. Rev. 2010, 254, 431455.

(283) Gibson, V. C.; Spitzmesser, S. K. Advances in NonMetallocene Olefin Polymerization Catalysis. Chem. Rev. 2003, 103, 283-315.

(284) Small, B. L. Discovery and Development of PyridineBis(imine) and Related Catalysts for Olefin Polymerization and Oligomerization. Acc. Chem. Res. 2015, 48, 2599-2611.

(285) Flisak, Z.; Sun, W. H. Progression of Diiminopyridines: From 
Single Application to Catalytic Versatility. ACS Catal. 2015, 5, 4713-4724.

(286) Peng, D.; Yan, X.; Yu, C.; Zhang, S.; Li, X. Transition Metal Complexes Bearing Tridentate Ligands for Precise Olefin Polymerization. Polym. Chem. 2016, 7, 2601-2634.

(287) Hoyt, J. M.; Schmidt, V. A.; Tondreau, A. M.; Chirik, P. J. Iron-Catalyzed Intermolecular [2+2] Cycloadditions of Unactivated Alkenes. Science 2015, 349, 960-963.

(288) Spahn, N. A.; Nguyen, M. H.; Renner, J.; Lane, T. K.; Louie, J. Regioselective Iron-Catalyzed $[2+2+2]$ Cycloaddition Reaction Forming 4,6-Disubstituted 2-Aminopyridines from Terminal Alkynes and Cyanamides. J. Org. Chem. 2017, 82, 234-242.

(289) Darmon, J. M.; Yu, R. P.; Semproni, S. P.; Turner, Z. R.; Stieber, S. C. E.; Debeer, S.; Chirik, P. J. Electronic Structure Determination of Pyridine N-Heterocyclic Carbene Iron Dinitrogen Complexes and Neutral Ligand Derivatives. Organometallics 2014, 33, 5423-5433.

(290) Sylvester, K. T.; Chirik, P. J. Iron-Catalyzed, HydrogenMediated Reductive Cyclization of 1,6-Enynes and Diynes: Evidence for Bis(imino)pyridine Ligand Participation. J. Am. Chem. Soc. 2009, 131, 8772-8774.

(291) Russell, S. K.; Lobkovsky, E.; Chirik, P. J. Iron-Catalyzed Intermolecular $[2 \pi+2 \pi]$ Cycloaddition. J. Am. Chem. Soc. 2011, 133, 8858-8861.

(292) Dsouza, B. R.; Lane, T. K.; Louie, J. Iron-Catalyzed Cycloaddition of Alkynenitriles and Alkynes. Org. Lett. 2011, 13, 2936-2939.

(293) Schmidt, V. A.; Hoyt, J. M.; Margulieux, G. W.; Chirik, P. J. Cobalt-Catalyzed $[2 \pi+2 \pi]$ Cycloadditions of Alkenes: Scope, Mechanism, and Elucidation of Electronic Structure of Catalytic Intermediates. J. Am. Chem. Soc. 2015, 137, 79037914.

(294) Werth, J.; Uyeda, C. Regioselective Simmons-Smith-Type Cyclopropanations of Polyalkenes Enabled by Transition Metal Catalysis. Chem. Sci. 2018, 1604-1609.

(295) Archer, A. M.; Bouwkamp, M. W.; Cortez, M.-P.; Lobkovsky, E.; Chirik, P. J. Arene Coordination in Bis(imino)pyridine Iron Complexes: Identification of Catalyst Deactivation Pathways in Iron-Catalyzed Hydrogenation and Hydrosilation. Organometallics 2006, 25, 4269-4278.

(296) Monfette, S.; Turner, Z. R.; Semproni, S. P.; Chirik, P. J. Enantiopure $\mathrm{C}_{1}$-Symmetric Bis(imino)pyridine Cobalt Complexes for Asymmetric Alkene Hydrogenation. J. Am. Chem. Soc. 2012, 134, 4561-4564.

(297) Bart, S. C.; Lobkovsky, E.; Bill, E.; Chirik, P. J. Synthesis and Hydrogenation of Bis(imino)pyridine Iron Imides. J. Am. Chem. Soc. 2006, 128, 5302-5303.

(298) Bart, S. C.; Lobkovsky, E.; Chirik, P. J. Preparation and Molecular and Electronic Structures of Iron(o) Dinitrogen and Silane Complexes and Their Application to Catalytic Hydrogenation and Hydrosilation. J. Am. Chem. Soc. 2oo4, 126, 13794-13807.

(299) Friedfeld, M. R.; Shevlin, M.; Margulieux, G. W.; Campeau, L. C.; Chirik, P. J. Cobalt-Catalyzed Enantioselective Hydrogenation of Minimally Functionalized Alkenes: Isotopic Labeling Provides Insight into the Origin of Stereoselectivity and Alkene Insertion Preferences. J. Am. Chem. Soc. 2016, 138, 3314-3324.

(30o) Atienza, C. C. H.; Diao, T.; Weller, K. J.; Nye, S. A.; Lewis, K. M.; Delis, J. G. P.; Boyer, J. L.; Roy, A. K.; Chirik, P. J. Bis(imino)pyridine Cobalt-Catalyzed Dehydrogenative Silylation of Alkenes: Scope, Mechanism, and Origins of Selective Allylsilane Formation. J. Am. Chem. Soc. 2014, 136, 12108-12118.

(301) Mukhopadhyay, T. K.; Flores, M.; Groy, T. L.; Trovitch, R. J. A Highly Active Manganese Precatalyst for the Hydrosilylation of Ketones and Esters. J. Am. Chem. Soc. 2014, 136, 882-885.

(302) Obligacion, J. V.; Chirik, P. J. Bis(imino)pyridine CobaltCatalyzed Alkene Isomerization-Hydroboration: A Strategy for Remote Hydrofunctionalization with Terminal Selectivity. J. Am. Chem. Soc. 2013, 135, 19107-19110.

(303) Luca, O. R.; Konezny, S. J.; Blakemore, J. D.; Colosi, D. M.; Saha, S.; Brudvig, G. W.; Batista, V. S.; Crabtree, R. H. A Tridentate Ni Pincer for Aqueous Electrocatalytic Hydrogen Production. New J. Chem. 2012, 36, 1149.

(304) Schroeder, M. A.; Wrighton, M. S. Pentacarbonyliron(o) Photocatalyzed Hydrogenation and Isomerization of Olefins. J. Am. Chem. Soc. 1976, 98, 551-558.

(305) A. Schroeder, M.; S. Wrighton, M. Pentacarbonyliron(o) Photocatalyzed Reactions of Trialkylsilanes with Alkenes. J. Organomet. Chem. 1977, 128, 345-358.

(306) Kismartoni, L. C.; Weitz, E.; Cedeño, D. L. Density Functional Study of $\mathrm{Fe}(\mathrm{CO})(3)$ and $\mathrm{Fe}(\mathrm{CO})(3)(\mathrm{L})$ with $\mathrm{H}-2$ and $\mathrm{C}_{2} \mathrm{H}_{4}$, Where $\mathrm{L}=\mathrm{H}_{2}$ or $\mathrm{C}_{2} \mathrm{H}_{4}$ : Reactions Relevant to Olefin Hydrogenation. Organometallics 2005, 24, 4714-4720.

(307) Whetten, R. L.; Fu, K. J.; Grant, E. R. Pulsed-Laser Photocatalytic Isomerization and Hydrogenation of Olefins. J. Am. Chem. Soc. 1982, 104, 4270-4272.

(308) Darmon, J. M.; Turner, Z. R.; Lobkovsky, E.; Chirik, P. J. Electronic Effects in 4-Substituted Bis(imino)pyridines and the Corresponding Reduced Iron Compounds. Organometallics 2012, 31, 2275-2285.

(309) Tondreau, A. M.; Darmon, J. M.; Wile, B. M.; Floyd, S. K.; Lobkovsky, E.; Chirik, P. J. Enantiopure Pyridine Bis(oxazoline) "pybox" and Bis(oxazoline) "box" iron Dialkyl Complexes: Comparison to Bis(imino)pyridine Compounds and Application to Catalytic Hydrosilylation of Ketones. Organometallics 2009, 28, 3928-3940.

(310) Thammavongsy, Z.; LeDoux, M. E.; Breuhaus-Alvarez, A. G.; Seda, T.; Zakharov, L. N.; Gilbertson, J. D. Pyridinediimine Iron Dicarbonyl Complexes with Pendant Lewis Bases and Lewis Acids Located in the Secondary Coordination Sphere. Eur. J. Inorg. Chem. 2013, 2013, 4008-4015.

(311) Thammavongsy, Z.; Seda, T.; Zakharov, L. N.; Kaminsky, W.; Gilbertson, J. D. Ligand-Based Reduction of $\mathrm{CO}_{2}$ and Release of CO on Iron(II). Inorg. Chem. 2012, 51, 9168-9170.

(312) Delgado, M.; Ziegler, J. M.; Seda, T.; Zakharov, L. N.; Gilbertson, J. D. Pyridinediimine Iron Complexes with Pendant Redox-Inactive Metals Located in the Secondary Coordination Sphere. Inorg. Chem. 2016, 55, 555-557.

(313) Russell, S. K.; Darmon, J. M.; Lobkovsky, E.; Chirik, P. J. Synthesis of Aryl-Substituted Bis(imino)pyridine Iron Dinitrogen Complexes. Inorg. Chem. 2o10, 49, 2782-2792.

（314） Burns, K. T.; Marks, W. R.; Cheung, P. M.; Seda, T.; Zakharov, L. N.; Gilbertson, J. D. Uncoupled Redox-Inactive Lewis Acids in the Secondary Coordination Sphere Entice LigandBased Nitrite Reduction. Inorg. Chem. 2018, 57, 9601-9610.

(315) Bart, S. C.; Bowman, A. C.; Lobkovsky, E.; Chirik, P. J. Iron Diazoalkane Chemistry: N-N Bond Hydrogenation and Intramolecular C-H Activation. J. Am. Chem. Soc. 2007, 129, 7212-7213.

(316) Russell, S. K.; Milsmann, C.; Lobkovsky, E.; Weyhermüller, T.; Chirik, P. J. Synthesis, Electronic Structure, and Catalytic Activity of Reduced Bis(aldimino)pyridine Iron Compounds: Experimental Evidence for Ligand Participation. Inorg. Chem. 2011, 50, 3159-3169.

(317) Bowman, A. C.; Milsmann, C.; Bill, E.; Lobkovsky, E.; Weyhermüller, T.; Wieghardt, K.; Chirik, P. J. Reduced N Alkyl Substituted Bis(imino)pyridine Cobalt Complexes: Molecular and Electronic Structures for Compounds Varying by Three Oxidation States. Inorg. Chem. 2010, 49, 6110-6123.

(318) Moyses Araujo, C.; Doherty, M. D.; Konezny, S. J.; Luca, O. R.; Usyatinsky, A.; Grade, H.; Lobkovsky, E.; Soloveichik, G. L.; Crabtree, R. H.; Batista, V. S. Tuning Redox Potentials of Bis(imino)pyridine Cobalt Complexes: An Experimental and Theoretical Study Involving Solvent and Ligand Effects. Dalton Trans. 2012, 41, 3562.

(319) Edwards, D. A.; Mahon, M. F.; Martin, W. R.; Molloy, K. C.; Fanwick, P. E.; Walton, R. A. Manganese(II) Complexes Containing the Tridentate Ligands 2,6-bis[1- 
(Phenylimino)ethyl]pyridine, $\quad \mathrm{L1}, \quad$ or $\quad 2,6-\mathrm{bis}[1-(4-$ Methoxyphenylimino)ethyl]pyridine, L2. The Molecular Structures of Five-Co-Ordinate [MnBr2L1] and the Zinc Analogue [ $\mathrm{ZnCl}_{2} \mathrm{L1} 1$. J. Chem. Soc. Dalton Trans. 1990, 3161.

(320) Reardon, D.; Aharonian, G.; Gambarotta, S.; Yap, G. P. A. Mono- and Zerovalent Manganese Alkyl Complexes Supported by the A, $\alpha^{\prime}$-Diiminato Pyridine Ligand: Alkyl Stabilization at the Expense of Catalytic Performance. Organometallics 2002, 21, 786-788.

(321) Mukhopadhyay, T. K.; Ghosh, C.; Flores, M.; Groy, T. L.; Trovitch, R. J. Hydrosilylation of Aldehydes and Formates Using a Dimeric Manganese Precatalyst. Organometallics 2017, 36, 3477-3483.

(322) Mukhopadhyay, T. K.; MacLean, N. L.; Gan, L.; Ashley, D. C.; Groy, T. L.; Baik, M. H.; Jones, A. K.; Trovitch, R. J. Carbon Dioxide Promoted H+ Reduction Using a Bis(imino)pyridine Manganese Electrocatalyst. Inorg. Chem. 2015, 54, 44754482.

(323) Ghosh, C.; Mukhopadhyay, T. K.; Flores, M.; Groy, T. L.; Trovitch, R. J. A Pentacoordinate Mn(II) Precatalyst That Exhibits Notable Aldehyde and Ketone Hydrosilylation Turnover Frequencies. Inorg. Chem. 2015, 54, 10398-10406.

(324) Sugiyama, H.; Aharonian, G.; Gambarotta, S.; Yap, G. P. a; Budzelaar, P. H. M. Participation of the A, $\alpha$ Diiminopyridine Ligand System in Reduction of the Metal Center during Alkylation. J. Am. Chem. Soc. 2002, 124, 1226812274.

(325) De Bruin, B.; Bill, E.; Bothe, E.; Weyhermüller, T.; Wieghardt, K. Molecular and Electronic Structures of Bis(pyridine-2,6Diimine)metal Complexes [ML2] (PF6)(n) $(\mathrm{N}=0,1,2,3 ; \mathrm{M}=$ $\mathrm{Mn}, \mathrm{Fe}, \mathrm{Co}, \mathrm{Ni}, \mathrm{Cu}, \mathrm{Zn})$. Inorg. Chem. 2ooo, 39, 2936-2947.

(326) Manuel, T. D.; Rohde, J. U. Reaction of a Redox-Active Ligand Complex of Nickel with Dioxygen Probes LigandRadical Character. J. Am. Chem. Soc. 2009, 131, 15582-15583.

(327) Reed, B. R.; Stoian, S. A.; Lord, R. L.; Groysman, S. The Aldimine Effect in Bis(imino)pyridine Complexes: NonPlanar nickel(I) Complexes of a Bis(aldimino)pyridine Ligand. Chem. Commun. 2015, 51, 6496-6499.

(328) Zhou, Y. Y.; Hartline, D. R.; Steiman, T. J.; Fanwick, P. E.; Uyeda, C. Dinuclear Nickel Complexes in Five States of Oxidation Using a Redox-Active Ligand. Inorg. Chem. 2014, 53, 11770-11777.

（329） Behlen, M. J.; Zhou, Y.-Y.; Steiman, T. J.; Pal, S.; Hartline, D. R.; Zeller, M.; Uyeda, C. Dinuclear Oxidative Addition Reactions Using an Isostructural Series of $\mathrm{Ni2}, \mathrm{Co} 2$, and $\mathrm{Fe} 2$ Complexes. Dalton Trans. 2017, 46, 5493-5497.

(330) Hirahara, M.; Yagi, M. Photoisomerization of ruthenium(II) Aquo Complexes: Mechanistic Insights and Application Development. Dalton Trans. 2017, 46, 3787-3799.

(331) Wong, K. M. C.; Yam, V. W. W. Luminescence platinum(II) Terpyridyl Complexes-From Fundamental Studies to Sensory Functions. Coord. Chem. Rev. 2007, 251, 2477-2488.

(332) Siebert, R.; Winter, A.; Schmitt, M.; Popp, J.; Schubert, U. S.; Dietzek, B. Light-Induced Dynamics in Conjugated Bis(terpyridine) Ligands - A Case Study toward Photoactive Coordination Polymers. Macromol. Rapid Commun. 2012, 33, 481-497.

(333) Wild, A.; Winter, A.; Schlütter, F.; Schubert, U. S. Advances in the Field of $\pi$-Conjugated $2,2^{\prime}: 6^{\prime}, 2^{\prime \prime}$-Terpyridines. Chem. Soc. Rev. 2011, 40, 1459-1511.

(334) Breivogel, A.; Kreitner, C.; Heinze, K. Redox and Photochemistry of bis(terpyridine)ruthenium(II) Amino Acids and Their Amide Conjugates - From Understanding to Applications. Eur. J. Inorg. Chem. 2014, 2014, 5468-549o.

(335) Cummings, S. D. Platinum Complexes of Terpyridine: Synthesis, Structure and Reactivity. Coord. Chem. Rev. 2oog, 253, 449-478.

(336) van Hilst, Q. V. C.; Lagesse, N. R.; Preston, D.; Crowley, J. D. Functional Metal Complexes from CuAAC "click" Bidentate and Tridentate Pyridyl-1,2,3-Triazole Ligands. Dalton Trans. 2018, 997-1002.
(337) Constable, E. C. $2,2^{\prime}: 6^{\prime}, \mathbf{2}^{\prime \prime}$-Terpyridines: From Chemical Obscurity to Common Supramolecular Motifs. Chem. Soc. Rev. 2007, 36, 246-253.

(338) Ziessel, R. Schiff-Based Bipyridine Ligands. Unusual Coordination Features and Mesomorphic Behaviour. Coord. Chem. Rev. 2001, 216-217, 195-223.

(339) Andres, P. R.; Schubert, U. S. New Functional Polymers and Materials Based on 2,2':6',2"-Terpyridine Metal Complexes. Adv. Mater. 2004, 16, 1043-1068.

(340) Winter, A.; Hoeppener, S.; Newkome, G. R.; Schubert, U. S. Terpyridine-Functionalized Surfaces: Redox-Active, Switchable, and Electroactive Nanoarchitecturesgland. Adv. Mater. 2011, 23, 3484-3498.

(341) Hayami, S.; Komatsu, Y.; Shimizu, T.; Kamihata, H.; Lee, Y. H. Spin-Crossover in cobalt(II) Compounds Containing Terpyridine and Its Derivatives. Coord. Chem. Rev. 2011, 255, 1981-1990.

(342) Budnikova, Y.; Vicic, D.; Klein, A. Exploring Mechanisms in Ni Terpyridine Catalyzed C-C Cross-Coupling Reactions-A Review. Inorganics 2018, 6, 18.

(343) Cherney, A. H.; Kadunce, N. T.; Reisman, S. E. Enantioselective and Enantiospecific Transition-MetalCatalyzed Cross-Coupling Reactions of Organometallic Reagents to Construct C-C Bonds. Chem. Rev. 2015, 115, 95879652.

（344） Hazari, N.; Melvin, P. R.; Beromi, M. M. Well-Defined Nickel and Palladium Precatalysts for Cross-Coupling. Nat. Rev. Chem. 2017, 1, 25.

(345) Hu, X. Nickel-Catalyzed Cross Coupling of Non-Activated Alkyl Halides: A Mechanistic Perspective. Chem. Sci. 2011, 2, 1867.

(346) Jana, R.; Pathak, T. P.; Sigman, M. S. Advances in Transition Metal (Pd,Ni,Fe)-Catalyzed Cross-Coupling Reactions Using Alkyl-Organometallics as Reaction Partners. Chem. Rev. 2011, 111, 1417-1492.

（347） England, J.; Scarborough, C. C.; Weyhermüller, T.; Sproules, S.; Wieghardt, K. Electronic Structures of the Electron Transfer Series [M(bpy) 3 ] N , [M(tpy) 2] N, and [Fe( T Bpy) 3] $\mathrm{N}\left(\mathrm{M}=\mathrm{Fe}, \mathrm{Ru} ; \mathrm{N}=3+, 2+, 1+, \mathrm{o}, 1^{-}\right):$A Mössbauer Spectroscopic and DFT Study. Eur. J. Inorg. Chem. 2012, 2012, 4605-4621.

(348) Morris, D. E.; Hanck, K. W.; Dearmond, M. K. Electrochemical and ESR Studies of [Fe(terpy)2]2+ and $\left[\mathrm{Ru}(\text { terpy })_{2}\right]_{2}+$ and Their Reduction Products. J. Electroanal. Chem. 1983, 149, 115-130.

(349) Hamacher, C.; Hurkes, N.; Kaiser, A.; Klein, A.; Schüren, A. Electrochemistry and Spectroscopy of Organometallic Terpyridine Nickel Complexes. Inorg. Chem. 2oo9, 48, 99479951.

(350) Scarborough, C. C.; Lancaster, K. M.; Debeer, S.; Weyhermu, T.; Sproules, S.; Wieghardt, K. Experimental Fingerprints for Redox-Active Terpyridine in $\left[\mathrm{Cr}(\mathrm{tpy})_{2}\right](\mathrm{PF} 6)$. Inorg. Chem. 2012, 51, 3718-3732.

(351) England, J.; Bill, E.; Weyhermüller, T.; Neese, F.; Atanasov, M.; Wieghardt, K. Molecular and Electronic Structures of Homoleptic Six-Coordinate Cobalt(I) Complexes of 2,2':6',2"'-Terpyridine, 2,2'-Bipyridine, and 1,10Phenanthroline. An Experimental and Computational Study. Inorg. Chem. 2015, 54, 12002-12018.

(352) Imler, G. H.; Lu, Z.; Kistler, K. A.; Carroll, P. J.; Wayland, B. B.; Zdilla, M. J. Complexes of 2,5-Bis( $\alpha$-Pyridyl)pyrrolate with $\mathrm{Pd}(\mathrm{II})$ and $\mathrm{Pt}(\mathrm{II})$ : A Monoanionic Iso- $\pi$-Electron Ligand Analog of Terpyridine. Inorg. Chem. 2012, 51, 10122-10128.

(353) Zhang, C. P.; Wang, H.; Klein, A.; Biewer, C.; Stirnat, K.; Yamaguchi, Y.; Xu, L.; Gomez-Benitez, V.; Vicic, D. A. A FiveCoordinate nickel(II) Fluoroalkyl Complex as a Precursor to a Spectroscopically Detectable Ni(III) Species. J. Am. Chem. Soc. 2013, 135, 8141-8144.

(354) Cabrero-Antonino, M.; Ballesteros-Garrido, R.; Chiassai, L.; Escrivà, E.; Ramirez de Arellano, C.; Ballesteros, R.; Abarca, B. Synthesis of New Terpyridine-like Ligands Based on 
Triazolopyridines and Benzotriazoles. New J. Chem. 2017, 41, 4918-4922.

(355) Harriman, K. L. M.; Leitch, A. A.; Stoian, S. A.; Habib, F.; Kneebone, J. L.; Gorelsky, S. I.; Korobkov, I.; Desgreniers, S.; Neidig, M. L.; Hill, S.; et al. Ambivalent Binding between a Radical-Based Pincer Ligand and Iron. Dalton Trans. 2015, 44, 10516-10523.

(356) Anderson, T. J.; Jones, G. D.; Vicic, D. A. Evidence for a Nil Active Species in the Catalytic Cross-Coupling of Alkyl Electrophiles. J. Am. Chem. Soc. 2004, 126, 8100-8101.

（357） Jones, G. D.; Martin, J. L.; McFarland, C.; Allen, O. R.; Hall, R. E.; Haley, A. D.; Brandon, R. J.; Konovalova, T.; Desrochers, P. J.; Pulay, P.; et al. Ligand Redox Effects in the Synthesis, Electronic Structure, and Reactivity of an Alkyl-Alkyl Cross-Coupling Catalyst. J. Am. Chem. Soc. 2006, 128, 13175-13183.

(358) Ciszewski, J. T.; Mikhaylov, D. Y.; Holin, K. V.; Kadirov, M. K.; Budnikova, Y. H.; Sinyashin, O.; Vicic, D. A. Redox Trends in Terpyridine Nickel Complexes. Inorg. Chem. 2011, 50, 8630-8635.

（359） Noviandri, I.; Brown, K. N.; Fleming, D. S.; Gulyas, P. T.; Lay, P. A.; Masters, A. F.; Phillips, L. The Decamethylferrocenium/Decamethylferrocene Redox Couple: A Superior Redox Standard to the Ferrocenium/Ferrocene Redox Couple for Studying Solvent Effects on the Thermodynamics of Electron Transfer. J. Phys. Chem. B 1999, 103, 6713-6722.

(360) Tondreau, A. M.; Atienza, C. C. H.; Darmon, J. M.; Milsmann, C.; Hoyt, H. M.; Weller, K. J.; Nye, S. A.; Lewis, K. M.; Boyer, J.; Delis, J. G. P.; et al. Synthesis, Electronic Structure, and Alkene Hydrosilylation Activity of Terpyridine and Bis(imino)pyridine Iron Dialkyl Complexes. Organometallics 2012, 31, 4886-4893.

(361) Palmer, W. N.; Diao, T.; Pappas, I.; Chirik, P. J. High-Activity Cobalt Catalysts for Alkene Hydroboration with Electronically Responsive Terpyridine and $\alpha$-Diimine Ligands. ACS Catal. 2015, 5, 622-626.

(362) Desimoni, G.; Faita, G.; Quadrelli, P. Pyridine-2,6Bis(oxazolines), Helpful Ligands for Asymmetric Catalysts. Chem. Rev. 2003, 103, 3119-3154.

(363) Desimoni, G.; Faita, G.; Jørgensen, K. A. C2-Symmetric Chiral Bis(oxazoline) Ligands in Asymmetric Catalysis. Chem. Rev. 2oo6, 106, 3561-3651.

(364) Kobayashi, S.; Yamashita, Y. Alkaline Earth Metal Catalysts for Asymmetric Reactions. Acc. Chem. Res. 2011, 44, 58-71.

(365) Singh, P. K.; Singh, V. K. Chiral 2,6-bis (5',5'diphenyloxazoline)pyridine as an Efficient Ligand for Asymmetric Catalysis. Pure Appl. Chem. 2012, 84, 1651-1657.

(366) Ward, B. D.; Gade, L. H. Rare Earth Metal Oxazoline Complexes in Asymmetric Catalysis. Chem. Commun. 2012, 48,10587 .

(367) Babu, S. A.; Krishnan, K. K.; Ujwaldev, S. M.; Anilkumar, G. Applications of Pybox Complexes in Asymmetric Catalysis. Asian J. Org. Chem. 2018, 7, 1033-1053.

(368) Schley, N. D.; Fu, G. C. Nickel-Catalyzed Negishi Arylations of Propargylic Bromides: A Mechanistic Investigation. J. Am. Chem. Soc. 2014, 136, 16588-16593.

(369) Andrew, R. E.; González-Sebastián, L.; Chaplin, A. B. NHCBased Pincer Ligands: Carbenes with a Bite. Dalton Trans. 2016, 45, 1299-1305.

(370) Danopoulos, A. A.; Wright, J. A.; Motherwell, W. B. Molecular $\mathrm{N}_{2}$ Complexes of Iron Stabilised by $\mathrm{N}$ Heterocyclic "pincer" Dicarbene Ligands. Chem. Commun. 2005, 41, 784-786.

(371) Danopoulos, A. A.; Pugh, D.; Smith, H.; Saßmannshausen, J. Structural and Reactivity Studies of "Pincer" Pyridine Dicarbene Complexes of $\mathrm{Fe} \mathrm{o}$ : Experimental and Computational Comparison of the Phosphine and NHC Donors. Chem. Eur. J. 2009, 15, 5491-5502.

(372) Danopoulos, A. A.; Wright, J. A.; Motherwell, W. B.; Ellwood, S. N-Heterocyclic "Pincer" Dicarbene Complexes of
Cobalt(I), Cobalt(II), and Cobalt(III). Organometallics 2004 23, 4807-4810.

(373) Yu, R. P.; Darmon, J. M.; Hoyt, J. M.; Margulieux, G. W.; Turner, Z. R.; Chirik, P. J. High-Activity Iron Catalysts for the Hydrogenation of Hindered, Unfunctionalized Alkenes. ACS Catal. 2012, 2, 1760-1764.

(374) Yu, R. P.; Darmon, J. M.; Milsmann, C.; Margulieux, G. W.; Stieber, S. C. E.; Debeer, S.; Chirik, P. J. Catalytic Hydrogenation Activity and Electronic Structure Determination of Bis(arylimidazol-2-Ylidene)pyridine Cobalt Alkyl and Hydride Complexes. J. Am. Chem. Soc. 2013, 135, 13168-13184.

(375) Khorobkov, I.; Gambarotta, S.; Yap, G. P. A.; Budzelaar, P. H. M. Reversible Alkylation at the Pyridine Nitrogen in a A, $\alpha-$ Diimine Pyridine Ligand System. Organometallics 2002, 21, 3088-3090.

（376） Lu, C. C.; Bill, E.; Weyhermüller, T.; Bothe, E.; Wieghardt, K. Neutral Bis( $\alpha$-Iminopyridine)metal Complexes of the FirstRow Transition Ions ( $\mathrm{Cr}, \mathrm{Mn}, \mathrm{Fe}, \mathrm{Co}, \mathrm{Ni}, \mathrm{Zn})$ and Their Monocationic Analogues: Mixed Valency Involving a Redox Noninnocent Ligand System. J. Am. Chem. Soc. 2008, 130, 3181-3197.

(377) Peng, D.; Zhang, Y.; Du, X.; Zhang, L.; Leng, X.; Walter, M. D.; Huang, Z. Phosphinite-Iminopyridine Iron Catalysts for Chemoselective Alkene Hydrosilylation. J. Am. Chem. Soc. 2013, 135, 19154-19166.

(378) DeRieux, W. S. W.; Wong, A.; Schrodi, Y. Synthesis and Characterization of Iron Complexes Based on BisPhosphinite PONOP and Bis-Phosphite PONOP Pincer Ligands. J. Organomet. Chem. 2014, 772-773, 6o-67.

(379) Du, X.; Zhang, Y.; Peng, D.; Huang, Z. Base-Metal-Catalyzed Regiodivergent Alkene Hydrosilylations. Angew. Chem. Int. Ed. 2016, 55, 6671-6675.

(380) Kaes, C.; Katz, A.; Hosseini, M. W. Bipyridine: The Most Widely Used Ligand. A Review of Molecules Comprising at Least Two 2,2'-Bipyridine Units. Chem. Rev. 2000, 100, 35533590.

(381) Zhang, L.; Zuo, Z.; Wan, X.; Huang, Z. Cobalt-Catalyzed Enantioselective Hydroboration of 1,1-Disubstituted Aryl Alkenes. J. Am. Chem. Soc. 2014, 136, 15501-15504.

(382) Chen, J.; Xi, T.; Lu, Z. Iminopyridine Oxazoline Iron Catalyst for Asymmetric Hydroboration of 1,1-Disubtituted Aryl Alkenes. Org. Lett. 2014, 16, 6452-6455.

(383) Chen, J.; Cheng, B.; Cao, M.; Lu, Z. Iron-Catalyzed Asymmetric Hydrosilylation of 1,1-Disubstituted Alkenes. Angew. Chem. Int. Ed. 2015, 54, 4661-4664.

(384) Chen, J.; Chen, C.; Ji, C.; Lu, Z. Cobalt-Catalyzed Asymmetric Hydrogenation of 1,1-Diarylethenes. Org. Lett. 2016, 18, 15941597.

(385) Ma, X.; Zuo, Z.; Liu, G.; Huang, Z. Manganese-Catalyzed Asymmetric Hydrosilylation of Aryl Ketones. ACS Omega 2017, 2, 4688-4692.

(386) Chen, X.; Cheng, Z.; Lu, Z. Iron-Catalyzed, MarkovnikovSelective Hydroboration of Styrenes. Org. Lett. 2017, 19, 969971.

(387) Scarborough, C. C.; Wieghardt, K. Electronic Structure of 2,2'-Bipyridine Organotransition-Metal Complexes. Establishing the Ligand Oxidation Level by Density Functional Theoretical Calculations. Inorg. Chem. 2011, 50, 9773-9793.

(388) Bowman, A. C.; Sproules, S.; Wieghardt, K. Electronic Structures of the [V( T Bpy) 3] Z ( $\mathrm{Z}=3+, 2+, 0,1-)$ Electron Transfer Series. Inorg. Chem. 2012, 51, 3707-3717.

(389) Irwin, M.; Doyle, L. R.; Krämer, T.; Herchel, R.; McGrady, J. E.; Goicoechea, J. M. A Homologous Series of First-Row Transition-Metal Complexes of 2,2'-bipyridine and Their Ligand Radical Derivatives: Trends in Structure, Magnetism, and Bonding. Inorg. Chem. 2012, 51, 12301-12312.

(390) Haines, R. J.; Wittrig, R. E.; Kubiak, C. P. Electrocatalytic Reduction of Carbon Dioxide by the Binuclear Copper Complex [Cu2(6-(Diphenylphosphino-2,2' 
bipyridyl)2(MeCN)2][PF6]2. Inorg. Chem. 1994, 33, 47234728.

(391) Zell, T.; Langer, R.; Iron, M. A.; Konstantinovski, L.; Shimon, L. J. W.; Diskin-Posner, Y.; Leitus, G.; Balaraman, E.; BenDavid, Y.; Milstein, D. Synthesis, Structures, and Dearomatization by Deprotonation of Iron Complexes Featuring Bipyridine-Based PNN Pincer Ligands. Inorg. Chem. 2013, 52, 9636-9649.

(392) Toya, Y.; Hayasaka, K.; Nakazawa, H. Hydrosilylation of Olefins Catalyzed by Iron Complexes Bearing Ketimine-Type Iminobipyridine Ligands. Organometallics 2017, 36, 17271735 .

(393) Kaim, W. Complexes with 2,2'-azobispyridine and Related "S-Frame" Bridging Ligands Containing the Azo Function. Coord. Chem. Rev. 2oo1, 219-221, 463-488.

(394) Waldie, K. M.; Ramakrishnan, S.; Kim, S. K.; Maclaren, J. K.; Chidsey, C. E. D.; Waymouth, R. M. Multielectron Transfer at Cobalt: Influence of the Phenylazopyridine Ligand. J. Am. Chem. Soc. 2017, 139, 4540-4550.

(395) Samanta, S.; Ghosh, P.; Goswami, S. Recent Advances on the Chemistry of Transition Metal Complexes of 2(Arylazo)pyridines and Its Arylamino Derivatives. Dalton Trans. 2012, 41, 2213.

(396) Ghosh, P.; Samanta, S.; Roy, S. K.; Demeshko, S.; Meyer, F.; Goswami, S. Introducing a New Azoaromatic Pincer Ligand. Isolation and Characterization of Redox Events in Its Ferrous Complexes. Inorg. Chem. 2014, 53, 4678-4686.

(397) Sinha, S.; Das, S.; Sikari, R.; Parua, S.; Brandaõ, P.; Demeshko, S.; Meyer, F.; Paul, N. D. Redox Noninnocent Azo-Aromatic Pincers and Their Iron Complexes. Isolation, Characterization, and Catalytic Alcohol Oxidation. Inorg. Chem. 2017, 56, 14084-14100.

(398) Polezhaev, A. V.; Chen, C.-H.; Kinne, A. S.; Cabelof, A. C.; Lord, R. L.; Caulton, K. G. Ligand Design toward Multifunctional Substrate Reductive Transformations. Inorg. Chem. 2017, 56, 9505-9514.

(399) Cook, B. J.; Pink, M.; Pal, K.; Caulton, K. G. Electron and Oxygen Atom Transfer Chemistry of $\mathrm{Co}(\mathrm{II})$ in a Proton Responsive, Redox Active Ligand Environment. Inorg. Chem. 2018, 57, 6176-6185.

(400) Polezhaev, A. V.; Liss, C. J.; Telser, J.; Chen, C.-H.; Caulton, K. G. A PNNH Pincer Ligand Allows Access to Monovalent Iron. Chem. Eur. J. 2018, 24, 1330-1341.

(401) Komine, N.; Buell, R. W.; Chen, C.-H.; Hui, A. K.; Pink, M.; Caulton, K. G. Probing the Steric and Electronic Characteristics of a New Bis-Pyrrolide Pincer Ligand. Inorg. Chem. 2014, 53, 1361-1369.

(402) Olguín, J.; Brooker, S. Spin Crossover Active iron(II) Complexes of Selected Pyrazole-Pyridine/pyrazine Ligands. Coord. Chem. Rev. 2011, 255, 203-240.

(403) Le Floch, P. Phosphaalkene, Phospholyl and Phosphinine Ligands: New Tools in Coordination Chemistry and Catalysis. Coord. Chem. Rev. 2006, 250, 627-681.

(404) Jouaiti, A.; Geoffroy, M.; Terron, G.; Bernardinelli, G. The Benzodiphosphaalkene Ligand and Its PdII and PtII Complexes: Their Synthesis, Structure, and an ESR Study of Their Reduction Products. J. Am. Chem. Soc. 1995, 117, 22512258.

(405) Nakajima, Y.; Nakao, Y.; Sakaki, S.; Tamada, Y.; Ono, T.; Ozawa, F. Electronic Structure of Four-Coordinate Iron(I) Complex Supported by a Bis(phosphaethenyl)pyridine Ligand. J. Am. Chem. Soc. 2o10, 132, 9934-9936.

(406) Nakajima, Y.; Ozawa, F. Redox Chemistry of Bis(phosphaethenyl)pyridine Iron Complexes. Organometallics 2012, 31, 2009-2015.

(407) Nakajima, Y.; Shiraishi, Y.; Tsuchimoto, T.; Ozawa, F. Synthesis and Coordination Behavior of CuI Bis(phosphaethenyl)pyridine Complexes. Chem. Commun. 2011, 47, 6332.

(408) Lin, Y.-F.; Ichihara, N.; Nakajima, Y.; Ozawa, F. Disproportionation of Bis(phosphaethenyl)pyridine Iron(I)
Bromide Induced by tBuNC. Organometallics 2014, 33, 67006703.

(409) Langer, R.; Iron, M. A.; Konstantinovski, L.; Diskin-Posner, Y.; Leitus, G.; Ben-David, Y.; Milstein, D. Iron Borohydride Pincer Complexes for the Efficient Hydrogenation of Ketones under Mild, Base-Free Conditions: Synthesis and Mechanistic Insight. Chem. Eur. J. 2012, 18, 7196-7209.

(410) Zell, T.; Ben-David, Y.; Milstein, D. Highly Efficient, General Hydrogenation of Aldehydes Catalyzed by PNP Iron Pincer Complexes. Catal. Sci. Technol. 2015, 5, 822-826.

(411) Yang, X. Unexpected Direct Reduction Mechanism for Hydrogenation of Ketones Catalyzed by Iron PNP Pincer Complexes. Inorg. Chem. 2011, 50, 12836-12843.

(412) Morello, G. R.; Hopmann, K. H. A Dihydride Mechanism Can Explain the Intriguing Substrate Selectivity of Iron-PNPMediated Hydrogenation. ACS Catal. 2017, 7, 5847-5855.

(413) Butschke, B.; Feller, M.; Diskin-Posner, Y.; Milstein, D. Ketone Hydrogenation Catalyzed by a New iron(II)-PNN Complex. Catal. Sci. Technol. 2016, 6, 4428-4437.

(414) Gorgas, N.; Stöger, B.; Veiros, L. F.; Pittenauer, E.; Allmaier, G.; Kirchner, K. Efficient Hydrogenation of Ketones and Aldehydes Catalyzed by Well-Defined Iron(II) PNP Pincer Complexes: Evidence for an Insertion Mechanism. Organometallics 2014, 33, 6905-6914.

(415) Schröder-Holzhacker, C.; Gorgas, N.; Stöger, B.; Kirchner, K. Synthesis and Reactivity of BINEPINE-Based Chiral Fe(II) PNP Pincer Complexes. Monatsh. Chem. 2016, 147, 10231030.

(416) Gorgas, N.; Stöger, B.; Veiros, L. F.; Kirchner, K. Highly Efficient and Selective Hydrogenation of Aldehydes: A WellDefined Fe(II) Catalyst Exhibits Noble-Metal Activity. ACS Catal. 2016, 6, 2664-2672.

(417) Kozuch, S.; Shaik, S. How to Conceptualize Catalytic Cycles? The Energetic Span Model. Acc. Chem. Res. 2011, 44, 101-110.

(418) Wodrich, M. D.; Busch, M.; Corminboeuf, C. Accessing and Predicting the Kinetic Profiles of Homogeneous Catalysts from Volcano Plots. Chem. Sci. 2016, 7, 5723-5735.

(419) Bruneau-Voisine, A.; Wang, D.; Roisnel, T.; Darcel, C.; Sortais, J.-B. Hydrogenation of Ketones with a Manganese $\mathrm{PN}^{3} \mathrm{P}$ Pincer Pre-Catalyst. Catal. Commun. 2017, 92, 1-4.

(420) Kallmeier, F.; Irrgang, T.; Dietel, T.; Kempe, R. Highly Active and Selective Manganese $\mathrm{C}=\mathrm{O}$ Bond Hydrogenation Catalysts: The Importance of the Multidentate Ligand, the Ancillary Ligands, and the Oxidation State. Angew. Chem. Int. Ed. 2016, 55, 11806-11809.

(421) Glatz, M.; Stöger, B.; Himmelbauer, D.; Veiros, L. F.; Kirchner, K. Chemoselective Hydrogenation of Aldehydes under Mild, Base-Free Conditions: Manganese Outperforms Rhenium. ACS Catal. 2018, 8, 4009-4016.

(422) Rösler, S.; Obenauf, J.; Kempe, R. A Highly Active and Easily Accessible Cobalt Catalyst for Selective Hydrogenation of $\mathrm{C}=\mathrm{O}$ Bonds. J. Am. Chem. Soc. 2015, 137, 7998-80o1.

(423) Lagaditis, P. O.; Sues, P. E.; Sonnenberg, J. F.; Wan, K. Y.; Lough, A. J.; Morris, R. H. Iron(II) Complexes Containing Unsymmetrical P-N-P' Pincer Ligands for the Catalytic Asymmetric Hydrogenation of Ketones and Imines. J. Am. Chem. Soc. 2014, 136, 1367-1380.

(424) Chakraborty, S.; Lagaditis, P. O.; Förster, M.; Bielinski, E. A.; Hazari, N.; Holthausen, M. C.; Jones, W. D.; Schneider, S. Well-Defined Iron Catalysts for the Acceptorless Reversible Dehydrogenation-Hydrogenation of Alcohols and Ketones. ACS Catal. 2014, 4, 3994-4003.

(425) Xia, T.; Wei, Z.; Spiegelberg, B.; Jiao, H.; Hinze, S.; de Vries, J. G. Isomerization of Allylic Alcohols to Ketones Catalyzed by Well-Defined Iron PNP Pincer Catalysts. Chem. Eur. J. 2018, 24, 4043-4049.

(426) Zhang, G.; Vasudevan, K. V.; Scott, B. L.; Hanson, S. K. Understanding the Mechanisms of Cobalt-Catalyzed Hydrogenation and Dehydrogenation Reactions. J. Am. Chem. Soc. 2013, 135, 8668-8681.

(427) Zhang, G.; Hanson, S. K. Cobalt-Catalyzed Transfer 
Hydrogenation of $\mathrm{C}=\mathrm{O}$ and $\mathrm{C}=\mathrm{N}$ Bonds. Chem. Commun. 2013, 49, 10151.

(428) Bäckvall, J.-E. Transition Metal Hydrides as Active Intermediates in Hydrogen Transfer Reactions. J. Organomet. Chem. 2002, 652, 105-111.

(429) Elangovan, S.; Topf, C.; Fischer, S.; Jiao, H.; Spannenberg, A.; Baumann, W.; Ludwig, R.; Junge, K.; Beller, M. Selective Catalytic Hydrogenations of Nitriles, Ketones, and Aldehydes by Well-Defined Manganese Pincer Complexes. J. Am. Chem. Soc. 2016, 138, 8809-8814.

(430) Li, H.; Wei, D.; Bruneau-Voisine, A.; Ducamp, M.; Henrion, M.; Roisnel, T.; Dorcet, V.; Darcel, C.; Carpentier, J.-F.; Soulé, J.-F.; et al. Rhenium and Manganese Complexes Bearing Amino-Bis(phosphinite) Ligands: Synthesis, Characterization, and Catalytic Activity in Hydrogenation of Ketones. Organometallics 2018, 37, 1271-1279.

(431) Perez, M.; Elangovan, S.; Spannenberg, A.; Junge, K.; Beller, M. Molecularly Defined Manganese Pincer Complexes for Selective Transfer Hydrogenation of Ketones. ChemSusChem 2017, 10, 83-86.

(432) Meyer, N.; Lough, A. J.; Morris, R. H. Iron(II) Complexes for the Efficient Catalytic Asymmetric Transfer Hydrogenation of Ketones. Chem. Eur. J. 2009, 15, 5605-5610.

(433) Sues, P. E.; Lough, A. J.; Morris, R. H. Stereoelectronic Factors in Iron Catalysis: Synthesis and Characterization of Aryl-Substituted Iron(II) Carbonyl P-N-N-P Complexes and Their Use in the Asymmetric Transfer Hydrogenation of Ketones. Organometallics 2011, 30, 4418-4431.

(434) Zuo, W.; Lough, A. J.; Li, Y. F.; Morris, R. H. Amine(imine)diphosphine Iron Catalysts for Asymmetric Transfer Hydrogenation of Ketones and Imines. Science 2013, 342, 1080-1083.

(435) Zuo, W.; Prokopchuk, D. E.; Lough, A. J.; Morris, R. H. Details of the Mechanism of the Asymmetric Transfer Hydrogenation of Acetophenone Using the Amine(imine)diphosphine Iron Precatalyst: The Base Effect and The Enantiodetermining Step. ACS Catal. 2016, 6, 301314 .

(436) Demmans, K. Z.; Seo, C. S. G.; Lough, A. J.; Morris, R. H. From Imine to Amine: An Unexpected Left Turn. Cis- $\beta$ iron(II) PNNP' Precatalysts for the Asymmetric Transfer Hydrogenation of Acetophenone. Chem. Sci. 2017, 8, 65316541.

(437) Smith, S. A. M.; Prokopchuk, D. E.; Morris, R. H. Asymmetric Transfer Hydrogenation of Ketones Using New Iron(II) (PNH-N-P') Catalysts: Changing the Steric and Electronic Properties at Phosphorus P'. Isr. J. Chem. 2017, 57, 1204-1215.

(438) Smith, S. A. M.; Lagaditis, P. O.; Lüpke, A.; Lough, A. J.; Morris, R. H. Unsymmetrical Iron P-NH-P' Catalysts for the Asymmetric Pressure Hydrogenation of Aryl Ketones. Chem. Eur. J. 2017, 23, 7212-7216.

(439) Sonnenberg, J. F.; Lough, A. J.; Morris, R. H. Synthesis of Iron $\mathrm{P}-\mathrm{N}-\mathrm{P}^{\prime}$ and $\mathrm{P}-\mathrm{NH}-\mathrm{P}^{\prime}$ Asymmetric Hydrogenation Catalysts. Organometallics 2014, 33, 6452-6465.

(440) Huber, R.; Passera, A.; Mezzetti, A. Iron(II)-Catalyzed Hydrogenation of Acetophenone with a Chiral, PyridineBased PNP Pincer Ligand: Support for an Outer-Sphere Mechanism. Organometallics 2018, 37, 396-405.

(441) Zirakzadeh, A.; Kirchner, K.; Roller, A.; Stöger, B.; Widhalm M.; Morris, R. H. Iron(II) Complexes Containing Chiral Unsymmetrical PNP' Pincer Ligands: Synthesis and Application in Asymmetric Hydrogenations. Organometallics 2016, 35, 3781-3787.

(442) Zirakzadeh, A.; de Aguiar, S. R. M. M.; Stöger, B.; Widhalm, M.; Kirchner, K. Enantioselective Transfer Hydrogenation of Ketones Catalyzed by a Manganese Complex Containing an Unsymmetrical Chiral PNP' Tridentate Ligand. ChemCatChem 2017, 9, 1744-1748.

(443) Widegren, M. B.; Harkness, G. J.; Slawin, A. M. Z.; Cordes, D. B.; Clarke, M. L. A Highly Active Manganese Catalyst for Enantioselective Ketone and Ester Hydrogenation. Angew.
Chem. Int. Ed. 2017, 56, 5825-5828.

(444) Garbe, M.; Junge, K.; Walker, S.; Wei, Z.; Jiao, H.; Spannenberg, A.; Bachmann, S.; Scalone, M.; Beller, M. Manganese(I)-Catalyzed Enantioselective Hydrogenation of Ketones Using a Defined Chiral PNP Pincer Ligand. Angew. Chem. Int. Ed. 2017, 56, 11237-11241.

(445) Zhou, S.; Fleischer, S.; Junge, K.; Beller, M. Cooperative Transition-Metal and Chiral Brønsted Acid Catalysis: Enantioselective Hydrogenation of Imines To Form Amines. Angew. Chem. Int. Ed. 2011, 50, 5120-5124.

(446) Lagaditis, P. O.; Lough, A. J.; Morris, R. H. Low-Valent EneAmido Iron Complexes for the Asymmetric Transfer Hydrogenation of Acetophenone without Base. J. Am. Chem. Soc. 2011, 133, 9662-9665.

(447) Xu, R.; Chakraborty, S.; Yuan, H.; Jones, W. D. Acceptorless, Reversible Dehydrogenation and Hydrogenation of $\mathrm{N}$ Heterocycles with a Cobalt Pincer Catalyst. ACS Catal. 2015, $5,6350-6354$.

(448) Garg, J. A.; Chakraborty, S.; Ben-David, Y.; Milstein, D. Unprecedented Iron-Catalyzed Selective Hydrogenation of Activated Amides to Amines and Alcohols. Chem. Commun. 2016, 52, 5285-5288.

(449) Zell, T.; Ben-David, Y.; Milstein, D. Unprecedented IronCatalyzed Ester Hydrogenation. Mild, Selective, and Efficient Hydrogenation of Trifluoroacetic Esters to Alcohols Catalyzed by an Iron Pincer Complex. Angew. Chem. Int. Ed. 2014, 53, 4685-4689.

(450) Elangovan, S.; Wendt, B.; Topf, C.; Bachmann, S.; Scalone, M.; Spannenberg, A.; Jiao, H.; Baumann, W.; Junge, K.; Beller, M. Improved Second Generation Iron Pincer Complexes for Effective Ester Hydrogenation. Adv. Synth. Catal. 2016, 358, 820-825.

(451) Rezayee, N. M.; Samblanet, D. C.; Sanford, M. S. IronCatalyzed Hydrogenation of Amides to Alcohols and Amines. ACS Catal. 2016, 6, 6377-6383.

(452) Jayarathne, U.; Zhang, Y.; Hazari, N.; Bernskoetter, W. H. Selective Iron-Catalyzed Deaminative Hydrogenation of Amides. Organometallics 2017, 36, 409-416.

(453) Artús Suàrez, L.; Culakova, Z.; Balcells, D.; Bernskoetter, W. H.; Eisenstein, O.; Goldberg, K. I.; Hazari, N.; Tilset, M.; Nova, A. The Key Role of the Hemiaminal Intermediate in the Iron-Catalyzed Deaminative Hydrogenation of Amides. ACS Catal. 2018, 8, 8751-8762.

(454) Dong, K.; Elangovan, S.; Sang, R.; Spannenberg, A.; Jackstell, R.; Junge, K.; Li, Y.; Beller, M. Selective Catalytic Two-Step Process for Ethylene Glycol from Carbon Monoxide. Nat. Commun. 2016, 7, 12075

(455) Zou, Y.-Q.; Chakraborty, S.; Nerush, A.; Oren, D.; DiskinPosner, Y.; Ben-David, Y.; Milstein, D. Highly Selective, Efficient Deoxygenative Hydrogenation of Amides Catalyzed by a Manganese Pincer Complex via Metal-Ligand Cooperation. ACS Catal. 2018, 8, 8014-8019.

(456) Papa, V.; Cabrero-Antonino, J. R.; Alberico, E.; Spanneberg, A.; Junge, K.; Junge, H.; Beller, M. Efficient and Selective Hydrogenation of Amides to Alcohols and Amines Using a Well-Defined manganese-PNN Pincer Complex. Chem. Sci. 2017, 8, 3576-3585.

(457) Bornschein, C.; Werkmeister, S.; Wendt, B.; Jiao, H.; Alberico, E.; Baumann, W.; Junge, H.; Junge, K.; Beller, M. Mild and Selective Hydrogenation of Aromatic and Aliphatic (Di)nitriles with a Well-Defined Iron Pincer Complex. Nat. Commun. 2014, 5, 1-11.

(458) Werkmeister, S.; Junge, K.; Wendt, B.; Alberico, E.; Jiao, H.; Baumann, W.; Junge, H.; Gallou, F.; Beller, M Hydrogenation of Esters to Alcohols with a Well-Defined Iron Complex. Angew. Chem. Int. Ed. 2014, 53, 8722-8726.

(459) Lange, S.; Elangovan, S.; Cordes, C.; Spannenberg, A.; Jiao, H.; Junge, H.; Bachmann, S.; Scalone, M.; Topf, C.; Junge, K.; et al. Selective Catalytic Hydrogenation of Nitriles to Primary Amines Using Iron Pincer Complexes. Catal. Sci. Technol. 2016, 6, 4768-4772. 
(46o) Chakraborty, S.; Milstein, D. Selective Hydrogenation of Nitriles to Secondary Imines Catalyzed by an Iron Pincer Complex. ACS Catal. 2017, 7, 3968-3972.

(461) Chakraborty, S.; Leitus, G.; Milstein, D. Iron-Catalyzed Mild and Selective Hydrogenative Cross-Coupling of Nitriles and Amines To Form Secondary Aldimines. Angew. Chem. Int. Ed. 2017, 56, 2074-2078.

(462) Mukherjee, A.; Srimani, D.; Chakraborty, S.; Ben-David, Y.; Milstein, D. Selective Hydrogenation of Nitriles to Primary Amines Catalyzed by a Cobalt Pincer Complex. J. Am. Chem. Soc. 2015, 137, 8888-8891.

(463) Shao, Z.; Fu, S.; Wei, M.; Zhou, S.; Liu, Q. Mild and Selective Cobalt-Catalyzed Chemodivergent Transfer Hydrogenation of Nitriles. Angew. Chem. Int. Ed. 2016, 55, 14653-14657.

(464) Fairweather, N. T.; Gibson, M. S.; Guan, H. Homogeneous Hydrogenation of Fatty Acid Methyl Esters and Natural Oils under Neat Conditions. Organometallics 2015, 34, 335-339.

(465) Elangovan, S.; Garbe, M.; Jiao, H.; Spannenberg, A.; Junge, K.; Beller, M. Hydrogenation of Esters to Alcohols Catalyzed by Defined Manganese Pincer Complexes. Angew. Chem. Int. Ed. 2016, 55, 15364-15368.

(466) Espinosa-Jalapa, N. A.; Nerush, A.; Shimon, L. J. W.; Leitus, G.; Avram, L.; Ben-David, Y.; Milstein, D. ManganeseCatalyzed Hydrogenation of Esters to Alcohols. Chem. Eur. J. 2017, 23, 5934-5938.

(467) Srimani, D.; Mukherjee, A.; Goldberg, A. F. G.; Leitus, G.; Diskin-Posner, Y.; Shimon, L. J. W.; Ben David, Y.; Milstein, D. Cobalt-Catalyzed Hydrogenation of Esters to Alcohols: Unexpected Reactivity Trend Indicates Ester Enolate Intermediacy. Angew. Chem. Int. Ed. 2015, 54, 12357-12360.

(468) Yuwen, J.; Chakraborty, S.; Brennessel, W. W.; Jones, W. D. Additive-Free Cobalt-Catalyzed Hydrogenation of Esters to Alcohols. ACS Catal. 2017, 7, 3735-3740.

(469) Appel, A. M.; Bercaw, J. E.; Bocarsly, A. B.; Dobbek, H.; DuBois, D. L.; Dupuis, M.; Ferry, J. G.; Fujita, E.; Hille, R. Kenis, P. J. A.; et al. Frontiers, Opportunities, and Challenges in Biochemical and Chemical Catalysis of $\mathrm{CO}_{2}$ Fixation. Chem. Rev. 2013, 113, 6621-6658.

(470) Glüer, A.; Schneider, S. Iron Catalyzed Hydrogenation and Electrochemical Reduction of $\mathrm{CO}_{2}$ : The Role of Functional Ligands. J. Organomet. Chem. 2018, 861, 159-173.

(471) Joó, F.; Laurenczy, G.; Nádasdi, L.; Elek, J. Homogeneous Hydrogenation of Aqueous Hydrogen Carbonate to Formate under Exceedingly Mild Conditions-a Novel Possibility of Carbon Dioxide Activation†. Chem. Commun. 1999, 35, 971972.

(472) Rivada-Wheelaghan, O.; Dauth, A.; Leitus, G.; DiskinPosner, Y.; Milstein, D. Synthesis and Reactivity of Iron Complexes with a New Pyrazine-Based Pincer Ligand, and Application in Catalytic Low-Pressure Hydrogenation of Carbon Dioxide. Inorg. Chem. 2015, 54, 4526-4538.

(473) Bertini, F.; Gorgas, N.; Stöger, B.; Peruzzini, M.; Veiros, L. F.; Kirchner, K.; Gonsalvi, L. Efficient and Mild Carbon Dioxide Hydrogenation to Formate Catalyzed by Fe(II) Hydrido Carbonyl Complexes Bearing 2,6(Diaminopyridyl)diphosphine Pincer Ligands. ACS Catal. 2016, 6, 2889-2893.

(474) Bertini, F.; Glatz, M.; Gorgas, N.; Stöger, B.; Peruzzini, M.; Veiros, L. F.; Kirchner, K.; Gonsalvi, L. Carbon Dioxide Hydrogenation Catalysed by Well-Defined Mn(I) PNP Pincer Hydride Complexes. Chem. Sci. 2017, 8, 5024-5029.

(475) Zhang, Y.; MacIntosh, A. D.; Wong, J. L.; Bielinski, E. A.; Williard, P. G.; Mercado, B. Q.; Hazari, N.; Bernskoetter, W. $\mathrm{H}$. Iron Catalyzed $\mathrm{CO} 2$ Hydrogenation to Formate Enhanced by Lewis Acid Co-Catalysts. Chem. Sci. 2015, 6, 4291-4299.

(476) Jayarathne, U.; Hazari, N.; Bernskoetter, W. H. Selective Iron-Catalyzed $\mathrm{N}$-Formylation of Amines Using Dihydrogen and Carbon Dioxide. ACS Catal. 2018, 8, 1338-1345.

(477) Spentzos, A. Z.; Barnes, C. L.; Bernskoetter, W. H. Effective Pincer Cobalt Precatalysts for Lewis Acid Assisted $\mathrm{CO}_{2}$
Hydrogenation. Inorg. Chem. 2016, 55, 8225-8233.

(478) Mills, M. R.; Barnes, C. L.; Bernskoetter, W. H. Influences of Bifunctional PNP-Pincer Ligands on Low Valent Cobalt Complexes Relevant to $\mathrm{CO} 2$ Hydrogenation. Inorg. Chem. 2018, 57, 1590-1597.

(479) Daw, P.; Chakraborty, S.; Leitus, G.; Diskin-Posner, Y.; BenDavid, Y.; Milstein, D. Selective N -Formylation of Amines with $\mathrm{H} 2$ and $\mathrm{CO}_{2}$ Catalyzed by Cobalt Pincer Complexes. ACS Catal. 2017, 7, 2500-2504.

(48o) Kar, S.; Goeppert, A.; Kothandaraman, J.; Prakash, G. K. S. Manganese-Catalyzed Sequential Hydrogenation of $\mathrm{CO}_{2}$ to Methanol via Formamide. ACS Catal. 2017, 7, 6347-6351.

(481) Trovitch, R. J.; Lobkovsky, E.; Bill, E.; Chirik, P. J. Functional Group Tolerance and Substrate Scope in Bis(imino)pyridine Iron Catalyzed Alkene Hydrogenation. Organometallics 2008, 27, 1470-1478.

(482) Trovitch, R. J.; Lobkovsky, E.; Chirik, P. J. Bis(diisopropylphosphino)pyridine Iron Dicarbonyl, Dihydride, and Silyl Hydride Complexes. Inorg. Chem. 2oo6, 45, 7252-726o.

(483) Knijnenburg, Q.; Horton, A. D.; Heijden, H. van der; Kooistra, T. M.; Hetterscheid, D. G. H.; Smits, J. M. M.; Bruin, B. de; Budzelaar, P. H. M.; Gal, A. W. Olefin Hydrogenation Using Diimine Pyridine Complexes of Co and Rh. J. Mol. Catal. A Chem. 2005, 232, 151-159.

(484) Knijnenburg, Q.; Hetterscheid, D.; Kooistra, T. M.; Budzelaar, P. H. M. The Electronic Structure of (Diiminopyridine)cobalt(I) Complexes. Eur. J. Inorg. Chem. 2004, 2004, 1204-1211.

(485) Hopmann, K. H. Cobalt-Bis(imino)pyridine-Catalyzed Asymmetric Hydrogenation: Electronic Structure, Mechanism, and Stereoselectivity. Organometallics 2013, 32, 6388-6399.

(486) Guo, N.; Hu, M.-Y.; Feng, Y.; Zhu, S.-F. Highly Efficient and Practical Hydrogenation of Olefins Catalyzed by in Situ Generated Iron Complex Catalysts. Org. Chem. Front. 2015, 2, 692-696.

(487) Zell, T.; Milko, P.; Fillman, K. L.; Diskin-Posner, Y.; Bendikov, T.; Iron, M. A.; Leitus, G.; Ben-David, Y.; Neidig, M. L.; Milstein, D. Iron Dicarbonyl Complexes Featuring Bipyridine-Based PNN Pincer Ligands with Short Interpyridine $\mathrm{C}-\mathrm{C}$ Bond Lengths: Innocent or Non-Innocent Ligand? Chem. Eur. J. 2014, 20, 4403-4413.

(488) van der Vlugt, J. I. Cooperative Catalysis with First-Row Late Transition Metals. Eur. J. Inorg. Chem. 2012, 2012, 363-375.

(489) Pugh, D.; Wells, N. J.; Evans, D. J.; Danopoulos, A. A. Reactions of "pincer" Pyridine Dicarbene Complexes of Fe(o) with Silanes. Dalton Trans. 20o9, No. 35, 7189.

(490) Yu, R. P.; Darmon, J. M.; Semproni, S. P.; Turner, Z. R.; Chirik, P. J. Synthesis of Iron Hydride Complexes Relevant to Hydrogen Isotope Exchange in Pharmaceuticals. Organometallics 2017, 36, 4341-4343.

(491) Tokmic, K.; Markus, C. R.; Zhu, L.; Fout, A. R. Well-Defined Cobalt(I) Dihydrogen Catalyst: Experimental Evidence for a $\mathrm{Co}(\mathrm{I}) / \mathrm{Co}(\mathrm{III})$ Redox Process in Olefin Hydrogenation. J. Am. Chem. Soc. 2016, 138, 11907-11913.

(492) Tokmic, K.; Fout, A. R. Alkyne Semihydrogenation with a Well-Defined Nonclassical Co-H 2 Catalyst: A H 2 Spin on Isomerization and E -Selectivity. J. Am. Chem. Soc. 2016, 138, 13700-13705.

(493) Zhang, G.; Yin, Z.; Tan, J. Cobalt(II)-Catalysed Transfer Hydrogenation of Olefins. RSC Adv. 2016, 6, 22419-22423.

(494) Jing, Y.; Chen, X.; Yang, X. Computational Mechanistic Study of the Hydrogenation and Dehydrogenation Reactions Catalyzed by Cobalt Pincer Complexes. Organometallics 2015, 34, 5716-5722.

(495) Lin, T.-P.; Peters, J. C. Boryl-Mediated Reversible H 2 Activation at Cobalt: Catalytic Hydrogenation, Dehydrogenation, and Transfer Hydrogenation. J. Am. Chem. Soc. 2013, 135, 15310-15313.

(496) Harman, W. H.; Peters, J. C. Reversible $\mathrm{H}_{2}$ Addition across a 
Nickel-Borane Unit as a Promising Strategy for Catalysis. J. Am. Chem. Soc. 2012, 134, 5080-5082.

(497) Li, Y.; Hou, C.; Jiang, J.; Zhang, Z.; Zhao, C.; Page, A. J.; Ke, Z. General H 2 Activation Modes for Lewis Acid-Transition Metal Bifunctional Catalysts. ACS Catal. 2016, 6, 1655-1662.

(498) Michaelides, I. N.; Dixon, D. J. Catalytic Stereoselective Semihydrogenation of Alkynes to $E$-Alkenes. Angew. Chem. Int. Ed. 2013, 52, 806-808.

(499) Srimani, D.; Diskin-Posner, Y.; Ben-David, Y.; Milstein, D. Iron Pincer Complex Catalyzed, Environmentally Benign, E -Selective Semi-Hydrogenation of Alkynes. Angew. Chem. Int. Ed. 2013, 52, 14131-14134.

(50o) Fu, S.; Chen, N. Y.; Liu, X.; Shao, Z.; Luo, S. P.; Liu, Q. LigandControlled Cobalt-Catalyzed Transfer Hydrogenation of Alkynes: Stereodivergent Synthesis of Z- and E-Alkenes. J. Am. Chem. Soc. 2016, 138, 8588-8594.

(501) Landge, V. G.; Pitchaimani, J.; Midya, S. P.; Subaramanian, M.; Madhu, V.; Balaraman, E. Phosphine-Free Cobalt Pincer Complex Catalyzed Z-Selective Semi-Hydrogenation of Unbiased Alkynes. Catal. Sci. Technol. 2018, 8, 428-433.

(502) Brzozowska, A.; Azofra, L. M.; Zubar, V.; Atodiresei, I.; Cavallo, L.; Rueping, M.; El-Sepelgy, O. Highly Chemo- and Stereoselective Transfer Semihydrogenation of Alkynes Catalyzed by a Stable, Well-Defined Manganese(II) Complex. ACS Catal. 2018, 8, 4103-4109.

(503) Vispute, T. P.; Zhang, H.; Sanna, A.; Xiao, R.; Huber, G. W. Renewable Chemical Commodity Feedstocks from Integrated Catalytic Processing of Pyrolysis Oils. Science 2010, 330, 1222-1227.

(504) Tuck, C. O.; Perez, E.; Horvath, I. T.; Sheldon, R. A.; Poliakoff, M. Valorization of Biomass: Deriving More Value from Waste. Science 2012, 337, 695-699.

(505) Trincado, M.; Banerjee, D.; Grützmacher, H. Molecular Catalysts for Hydrogen Production from Alcohols. Energy Environ. Sci. 2014, 7, 2464-2503.

(506) Alberico, E.; Sponholz, P.; Cordes, C.; Nielsen, M.; Drexler, H.-J.; Baumann, W.; Junge, H.; Beller, M. Selective Hydrogen Production from Methanol with a Defined Iron Pincer Catalyst under Mild Conditions. Angew. Chem. Int. Ed. 2013, 52, 14162-14166.

(507) Bielinski, E. A.; Förster, M.; Zhang, Y.; Bernskoetter, W. H.; Hazari, N.; Holthausen, M. C. Base-Free Methanol Dehydrogenation Using a Pincer-Supported Iron Compound and Lewis Acid Co-Catalyst. ACS Catal. 2015, 5, 2404-2415.

(508) Bonitatibus, P. J.; Chakraborty, S.; Doherty, M. D.; Siclovan, O.; Jones, W. D.; Soloveichik, G. L. Reversible Catalytic Dehydrogenation of Alcohols for Energy Storage. Proc. Natl. Acad. Sci. 2015, 112, 1687-1692.

(509) Sharninghausen, L. S.; Mercado, B. Q.; Crabtree, R. H.; Hazari, N. Selective Conversion of Glycerol to Lactic Acid with Iron Pincer Precatalysts. Chem. Commun. 2015, 51, 16201-16204.

(510) Peña-López, M.; Neumann, H.; Beller, M. Iron(II) PincerCatalyzed Synthesis of Lactones and Lactams through a Versatile Dehydrogenative Domino Sequence. ChemCatChem 2015, 7, 865-871.

(511) Andérez-Fernández, M.; Vogt, L. K.; Fischer, S.; Zhou, W.; Jiao, H.; Garbe, M.; Elangovan, S.; Junge, K.; Junge, H.; Ludwig, R.; et al. A Stable Manganese Pincer Catalyst for the Selective Dehydrogenation of Methanol. Angew. Chem. Int. Ed. 2017, 56, 559-562.

(512) Nguyen, D. H.; Morin, Y.; Zhang, L.; Trivelli, X.; Capet, F.; Paul, S.; Desset, S.; Dumeignil, F.; Gauvin, R. M. Oxidative Transformations of Biosourced Alcohols Catalyzed by EarthAbundant Transition Metals. ChemCatChem 2017, 9, 26522660 .

(513) Chakraborty, M.; Sengupta, D.; Saha, T.; Goswami, S. Ligand Redox-Controlled Tandem Synthesis of Azines from Aromatic Alcohols and Hydrazine in Air: One-Pot Synthesis of Phthalazine. J. Org. Chem. 2018, 83, 7771-7778.

(514) Jr, L. Q.; Tolman, W. B. Biologically Inspired Oxidation
Catalysis. 2008, 455, 333-340.

(515) Yi, H.; Jutand, A.; Lei, A. Evidence for the Interaction between $\mathrm{tBuOK}$ and 1,10-Phenanthroline to Form the 1,10Phenanthroline Radical Anion: A Key Step for the Activation of Aryl Bromides by Electron Transfer. Chem. Commun. 2015, $51,545-548$.

(516) Weinberg, D. R.; Gagliardi, C. J.; Hull, J. F.; Murphy, C. F.; Kent, C. A.; Westlake, B. C.; Paul, A.; Ess, D. H.; McCafferty, D. G.; Meyer, T. J. Proton-Coupled Electron Transfer. Chem. Rev. 2012, 112, 4016-4093.

(517) Cukier, R. I.; Nocera, D. G. Proton-Coupled Electron Transfer. Annu. Rev. Phys. Chem. 1998, 49, 337-369.

(518) Huynh, M. H. V.; Meyer, T. J. Proton-Coupled Electron Transfer. Chem. Rev. 2007, 107, 5004-5064.

(519) Boddien, A.; Loges, B.; Gärtner, F.; Torborg, C.; Fumino, K.; Junge, H.; Ludwig, R.; Beller, M. Iron-Catalyzed Hydrogen Production from Formic Acid. J. Am. Chem. Soc. 2010, 132, 8924-8934.

(520) Boddien, A.; Gärtner, F.; Jackstell, R.; Junge, H.; Spannenberg, A.; Baumann, W.; Ludwig, R.; Beller, M. Ortho-Metalation of Iron(o) Tribenzylphosphine Complexes: Homogeneous Catalysts for the Generation of Hydrogen from Formic Acid. Angew. Chem. Int. Ed. 2010, 49, 8993-8996.

(521) Boddien, A.; Mellmann, D.; Gartner, F.; Jackstell, R.; Junge, H.; Dyson, P. J.; Laurenczy, G.; Ludwig, R.; Beller, M. Efficient Dehydrogenation of Formic Acid Using an Iron Catalyst. Science 2011, 333, 1733-1736.

(522) Zell, T.; Butschke, B.; Ben-David, Y.; Milstein, D. Efficient Hydrogen Liberation from Formic Acid Catalyzed by a WellDefined Iron Pincer Complex under Mild Conditions. Chem. Eur. J. 2013, 19, 8068-8072.

(523) Mellone, I.; Gorgas, N.; Bertini, F.; Peruzzini, M.; Kirchner K.; Gonsalvi, L. Selective Formic Acid Dehydrogenation Catalyzed by Fe-PNP Pincer Complexes Based on the 2,6Diaminopyridine Scaffold. Organometallics 2016, 35, 33443349 .

(524) Marder, T. B. Will We Soon Be Fueling Our Automobiles with Ammonia-Borane? Angew. Chem. Int. Ed. 2007, 46, 8116-8118.

(525) Staubitz, A.; Robertson, A. P. M.; Manners, I. AmmoniaBorane and Related Compounds as Dihydrogen Sources. Chem. Rev. 2010, 110, 4079-4124.

(526) Staubitz, A.; Robertson, A. P. M.; Sloan, M. E.; Manners, I. Amine- and Phosphine-Borane Adducts: New Interest in Old Molecules. Chem. Rev. 2010, 110, 4023-4078.

(527) Glüer, A.; Förster, M.; Celinski, V. R.; Schmedt auf der Günne, J.; Holthausen, M. C.; Schneider, S. Highly Active Iron Catalyst for Ammonia Borane Dehydrocoupling at Room Temperature. ACS Catal. 2015, 5, 7214-7217.

(528) Anke, F.; Han, D.; Klahn, M.; Spannenberg, A.; Beweries, T. Formation of High-Molecular Weight Polyaminoborane by Fe Hydride Catalysed Dehydrocoupling of Methylamine Borane. Dalton Trans. 2017, 46, 6843-6847.

(529) Bhattacharya, P.; Krause, J. A.; Guan, H. Mechanistic Studies of Ammonia Borane Dehydrogenation Catalyzed by Iron Pincer Complexes. J. Am. Chem. Soc. 2014, 136, 11153-11161.

(530) Woods, A.; Rahman, K. M. Antimicrobial Molecules in the Lung: Formulation Challenges and Future Directions for Innovation. Future Med. Chem. 2018, 10, 575-604.

(531) Sarkar, P.; Yarlagadda, V.; Ghosh, C.; Haldar, J. A Review on Cell Wall Synthesis Inhibitors with an Emphasis on Glycopeptide Antibiotics. MedChemComm 2017, 8, 516-533.

(532) Mehta, P. D.; Sengar, N. P. S.; Pathak, A. K. 2-Azetidinone A New Profile of Various Pharmacological Activities. Eur. J. Med. Chem. 2010, 45, 5541-556o.

（533） Lane, E. M.; Uttley, K. B.; Hazari, N.; Bernskoetter, W. IronCatalyzed Amide Formation from the Dehydrogenative Coupling of Alcohols and Secondary Amines. Organometallics 2017, 36, 2020-2025.

(534) Chakraborty, S.; Gellrich, U.; Diskin-Posner, Y.; Leitus, G.; 
Avram, L.; Milstein, D. Manganese-Catalyzed N-Formylation of Amines by Methanol Liberating $\mathrm{H}_{2}$ : A Catalytic and Mechanistic Study. Angew. Chem. Int. Ed. 2017, 56, 42294233.

(535) Bauer, J. O.; Chakraborty, S.; Milstein, D. ManganeseCatalyzed Direct Deoxygenation of Primary Alcohols. ACS Catal. 2017, 7, 4462-4466.

(536) Chakraborty, S.; Das, U. K.; Ben-David, Y.; Milstein, D. Manganese Catalyzed $\alpha$-Olefination of Nitriles by Primary Alcohols. J. Am. Chem. Soc. 2017, 139, 11710-11713.

(537) Bordwell, F. G.; Cheng, J.-P.; Bausch, M. J.; Bares, J. E. Acidities of Radical Cations Derived from Arylacetonitriles. J. Phys. Org. Chem. 1988, 1, 209-223.

(538) Espinosa-Jalapa, N. A.; Kumar, A.; Leitus, G.; Diskin-Posner, Y.; Milstein, D. Synthesis of Cyclic Imides by Acceptorless Dehydrogenative Coupling of Diols and Amines Catalyzed by a Manganese Pincer Complex. J. Am. Chem. Soc. 2017, 139, 11722-11725.

(539) Kumar, A.; Espinosa-Jalapa, N. A.; Leitus, G.; Diskin-Posner, Y.; Avram, L.; Milstein, D. Direct Synthesis of Amides by Dehydrogenative Coupling of Amines with Either Alcohols or Esters: Manganese Pincer Complex as Catalyst. Angew. Chem. Int. Ed. 2017, 56, 14992-14996.

(540) Daw, P.; Chakraborty, S.; Garg, J. A.; Ben-David, Y.; Milstein, D. Direct Synthesis of Pyrroles by Dehydrogenative Coupling of Diols and Amines Catalyzed by Cobalt Pincer Complexes. Angew. Chem. Int. Ed. 2016, 55, 14373-14377.

(541) Daw, P.; Ben-David, Y.; Milstein, D. Direct Synthesis of Benzimidazoles by Dehydrogenative Coupling of Aromatic Diamines and Alcohols Catalyzed by Cobalt. ACS Catal. 2o17, 7, 7456-746o.

(542) Midya, S. P.; Landge, V. G.; Sahoo, M. K.; Rana, J.; Balaraman, E. Cobalt-Catalyzed Acceptorless Dehydrogenative Coupling of Aminoalcohols with Alcohols: Direct Access to Pyrrole, Pyridine and Pyrazine Derivatives. Chem. Commun. 2018, 54, 90-93.

(543) Daw, P.; Kumar, A.; Espinosa-Jalapa, N. A.; Diskin-Posner, Y.; Ben-David, Y.; Milstein, D. Synthesis of Pyrazines and Quinoxalines via Acceptorless Dehydrogenative Coupling Routes Catalyzed by Manganese Pincer Complexes. ACS Catal. 2018, 8, 7734-7741.

(544) Kallmeier, F.; Dudziec, B.; Irrgang, T.; Kempe, R. Manganese-Catalyzed Sustainable Synthesis of Pyrroles from Alcohols and Amino Alcohols. Angew. Chem. Int. Ed. 2017, 56, 7261-7265.

(545) Mastalir, M.; Glatz, M.; Pittenauer, E.; Allmaier, G.; Kirchner, K. Sustainable Synthesis of Quinolines and Pyrimidines Catalyzed by Manganese PNP Pincer Complexes. J. Am. Chem. Soc. 2016, 138, 15543-15546.

(546) Mastalir, M.; Pittenauer, E.; Allmaier, G.; Kirchner, K. Manganese-Catalyzed Aminomethylation of Aromatic Compounds with Methanol as a Sustainable $\mathrm{C}_{1}$ Building Block. J. Am. Chem. Soc. 2017, 139, 8812-8815.

(547) Hamid, M. H. S. A.; Slatford, P. A.; Williams, J. M. J. Borrowing Hydrogen in the Activation of Alcohols. Adv. Synth. Catal. 2007, 349, 1555-1575.

(548) Nixon, T. D.; Whittlesey, M. K.; Williams, J. M. J. Transition Metal Catalysed Reactions of Alcohols Using Borrowing Hydrogen Methodology. Dalton Trans. 2009, o, 753-762.

(549) Ketcham, J. M.; Shin, I.; Montgomery, T. P.; Krische, M. J. Catalytic Enantioselective C-H Functionalization of Alcohols by Redox-Triggered Carbonyl Addition: Borrowing Hydrogen, Returning Carbon. Angew. Chem. Int. Ed. 2014, 53, 9142-9150.

(550) Obora, Y. Recent Advances in $\alpha$ - Alkylation Reactions Using Alcohols with Hydrogen Borrowing Methodologies. ACS Catal. 2014, 4, 3972-3981.

(551) Quintard, A.; Rodriguez, J. A Step into an Eco-Compatible Future: Iron- and Cobalt-Catalyzed Borrowing Hydrogen Transformation. ChemSusChem 2016, 9, 28-30.

(552) Huang, F.; Liu, Z.; Yu, Z. C-Alkylation of Ketones and Related
Compounds by Alcohols: Transition-Metal-Catalyzed Dehydrogenation. Angew. Chem. Int. Ed. 2016, 55, 862-875.

(553) Corma, A.; Navas, J.; Sabater, M. J. Advances in One-Pot Synthesis through Borrowing Hydrogen Catalysis. Chem. Rev. 2018, 118, 1410-1459.

(554) Zhang, G.; Hanson, S. K. Cobalt-Catalyzed Acceptorless Alcohol Dehydrogenation: Synthesis of Imines from Alcohols and Amines. Org. Lett. 2013, 15, 650-653.

(555) Zhang, G.; Yin, Z.; Zheng, S. Cobalt-Catalyzed N-Alkylation of Amines with Alcohols. Org. Lett. 2016, 18, 300-303.

(556) Yin, Z.; Zeng, H.; Wu, J.; Zheng, S.; Zhang, G. CobaltCatalyzed Synthesis of Aromatic, Aliphatic, and Cyclic Secondary Amines via a "Hydrogen-Borrowing" Strategy. ACS Catal. 2016, 6, 6546-6550.

(557) Zhang, G.; Wu, J.; Zeng, H.; Zhang, S.; Yin, Z.; Zheng, S. Cobalt-Catalyzed $\alpha$-Alkylation of Ketones with Primary Alcohols. Org. Lett. 2017, 19, 1080-1083.

(558) Rösler, S.; Ertl, M.; Irrgang, T.; Kempe, R. Cobalt-Catalyzed Alkylation of Aromatic Amines by Alcohols. Angew. Chem. Int. Ed. 2015, 54, 15046-15050.

(559) Freitag, F.; Irrgang, T.; Kempe, R. Cobalt-Catalyzed Alkylation of Secondary Alcohols with Primary Alcohols via Borrowing Hydrogen/Hydrogen Autotransfer. Chem. Eur. J. 2017, 23, 12110-12113.

(56o) Deibl, N.; Kempe, R. General and Mild Cobalt-Catalyzed CAlkylation of Unactivated Amides and Esters with Alcohols. J. Am. Chem. Soc. 2016, 138, 10786-10789.

(561) Mastalir, M.; Glatz, M.; Gorgas, N.; Stöger, B.; Pittenauer, E.; Allmaier, G.; Veiros, L. F.; Kirchner, K. Divergent Coupling of Alcohols and Amines Catalyzed by Isoelectronic Hydride Mn I and Fe II PNP Pincer Complexes. Chem. Eur. J. 2016, 22, 12316-12320.

(562) Mastalir, M.; Stöger, B.; Pittenauer, E.; Puchberger, M.; Allmaier, G.; Kirchner, K. Air Stable Iron(II) PNP Pincer Complexes as Efficient Catalysts for the Selective Alkylation of Amines with Alcohols. Adv. Synth. Catal. 2016, 358, 38243831.

(563) Elangovan, S.; Neumann, J.; Sortais, J.-B.; Junge, K.; Darcel, C.; Beller, M. Efficient and Selective N-Alkylation of Amines with Alcohols Catalysed by Manganese Pincer Complexes. Nat. Commun. 2016, 7, 12641.

(564) Neumann, J.; Elangovan, S.; Spannenberg, A.; Junge, K.; Beller, M. Improved and General Manganese-Catalyzed NMethylation of Aromatic Amines Using Methanol. Chem. Eur. J. 2017, 23, 5410-5413.

(565) Bruneau-Voisine, A.; Wang, D.; Dorcet, V.; Roisnel, T.; Darcel, C.; Sortais, J.-B. Mono- N -Methylation of Anilines with Methanol Catalyzed by a Manganese Pincer-Complex. J. Catal. 2017, 347, 57-62.

(566) Peña-López, M.; Piehl, P.; Elangovan, S.; Neumann, H.; Beller, M. Manganese-Catalyzed Hydrogen-Autotransfer C-C Bond Formation: $\alpha$-Alkylation of Ketones with Primary Alcohols. Angew. Chem. Int. Ed. 2016, 55, 14967-14971.

(567) Fu, S.; Shao, Z.; Wang, Y.; Liu, Q. Manganese-Catalyzed Upgrading of Ethanol into 1-Butanol. J. Am. Chem. Soc. 2o17, 139, 11941-11948.

(568) Kulkarni, N. V.; Brennessel, W. W.; Jones, W. D. Catalytic Upgrading of Ethanol to N-Butanol via ManganeseMediated Guerbet Reaction. ACS Catal. 2o18, 8, 997-1002.

(569) Das, U. K.; Ben-David, Y.; Diskin-Posner, Y.; Milstein, D. NSubstituted Hydrazones by Manganese-Catalyzed Coupling of Alcohols with Hydrazine: Borrowing Hydrogen and Acceptorless Dehydrogenation in One System. Angew. Chem. Int. Ed. 2018, 57, 2179-2182.

(570) Barman, M. K.; Jana, A.; Maji, B. Phosphine-Free NNNManganese Complex Catalyzed $\alpha$-Alkylation of Ketones with Primary Alcohols and Friedländer Quinoline Synthesis. $A d v$. Synth. Catal. 2018. 


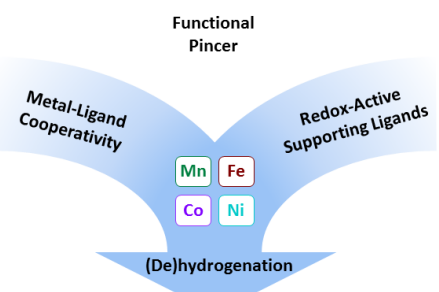

